\title{
Broadcast and network optimization : essays on approximation algorithms and experiments on problems in latency cover, network fragmentation and routing on the internet
}

Citation for published version (APA):

van der Zwaan, G. R. J. (2012). Broadcast and network optimization : essays on approximation algorithms and experiments on problems in latency cover, network fragmentation and routing on the internet. [Doctoral Thesis, Maastricht University]. Maastricht University. https://doi.org/10.26481/dis.20121102gz

Document status and date:

Published: 01/01/2012

DOI:

10.26481/dis.20121102gz

Document Version:

Publisher's PDF, also known as Version of record

Please check the document version of this publication:

- A submitted manuscript is the version of the article upon submission and before peer-review. There can be important differences between the submitted version and the official published version of record. People interested in the research are advised to contact the author for the final version of the publication, or visit the DOI to the publisher's website.

- The final author version and the galley proof are versions of the publication after peer review.

- The final published version features the final layout of the paper including the volume, issue and page numbers.

Link to publication

\footnotetext{
General rights rights.

- You may freely distribute the URL identifying the publication in the public portal. please follow below link for the End User Agreement:

www.umlib.nl/taverne-license

Take down policy

If you believe that this document breaches copyright please contact us at:

repository@maastrichtuniversity.nl

providing details and we will investigate your claim.
}

Copyright and moral rights for the publications made accessible in the public portal are retained by the authors and/or other copyright owners and it is a condition of accessing publications that users recognise and abide by the legal requirements associated with these

- Users may download and print one copy of any publication from the public portal for the purpose of private study or research.

- You may not further distribute the material or use it for any profit-making activity or commercial gain

If the publication is distributed under the terms of Article $25 \mathrm{fa}$ of the Dutch Copyright Act, indicated by the "Taverne" license above, 


$$
\text { G.R.J. VAN DER ZWAAN }
$$

\section{BROADCAST AND NETWORK OPTIMIZATION}


Broadcast and Network optimization by G.R.J. van der Zwaan.

E-MAIL: rubenvanderzwaan@gmail.com

The cover is art by Wilma Wessels. This book was typeset by the author using LaTex and the ClassicThesis and ArsClassica packages. 


\title{
BROADCAST AND NETWORK OPTIMIZATION
}

Essays on approximation algorithms and experiments on problems in latency cover, network fragmentation and routing on the internet

\section{Proefschrift}

\author{
ter verkrijging van de graad van doctor \\ aan de Universiteit Maastricht op gezag van \\ Rector Magnificus, Prof. Dr. L.L.G. Soete, \\ volgens het besluit van het College van Decanen, in het \\ openbaar te verdedigen op \\ vrijdag 2 november 2012 om 14.00 uur
}

door

\section{GIJSBERT RUBEN JOHAN VAN DER ZWAAN}


Promotor:

Prof. Dr. Ir. C.P.M. van Hoesel

Copromotor:

Dr. A. Berger

Beoordelingscommissie:

Prof. Dr. Ir. A.M.H. Gerards (Maastricht University / CWI)

Dr. S. Kelk (Maastricht University)

Prof. Dr. M. Skutella (Technische Universität Berlin) 


\section{CONTENTS}

I INTRODUCTION I

1.1 Latency covering problems I

1.2 Routing on the Internet 3

1.3 Connectivity in graphs 4

1.4 Computational Complexity 4

1.5 Thesis overview 7

1.6 Publications underlying this thesis 8

2 PRELIMINARIES 9

2.1 Basic notation 9

2.2 Computational complexity 10

2.3 Approximation algorithms 12

2.4 Unique Games Conjecture 13

2.5 Linear programming 14

2.6 Submodularity 16

2.7 Matroids 17

I MINIMUM LATENCY COVERING 19

3 LATENCY COVERING: INTRODUCTION 2 I

4 GENERALIZED MIN SUM SET COVER 29

4.1 Introduction 30

4.2 2-Approximation for Preemptive

Generalized Min Sum Set Cover 32

4.3 Hardness of approximation 48

4.4 Gap between Preemptive and Non-preemptive Schedules 51

4.5 Comparison of linear programming formulations $\quad 58$

4.6 Conclusions 63

5 MINIMUM LATENCY SUBMODULAR COVER 65

5.1 Introduction 66

5.2 Simpler Analysis of the Submodular Ranking Problem 69

5.3 Minimum Latency Submodular Cover 74

5.4 Weighted Stochastic Submodular Ranking $\quad 78$

5.5 Conclusions 86

6 LATENCY COVERING STEINER TREE 87

6.1 Introduction 88 
6.2 New LP Relaxation for Covering Steiner Tree 89

6.3 Latency Covering Steiner Tree Problem 98

II ROUTING AND CONNECTIVITY 105

7 PATH TRADING 107

7.1 Introduction 107

7.2 Model and Notation 110

7.3 Complexity Results and Smoothed Analysis

111

7.4 Evaluation 120

7.5 Conclusions 124

8 HOW TO CUT A GRAPH 127

8.1 Introduction 127

8.2 Hardness Results 129

8.3 Polynomial Cases 132

8.4 Fixed-parameter tractability and an EPTAS for Planar Graphs 136

8.5 Conclusions 145

BIBLIOGRAPHY 147

A NEDERLANDSE SAMENVATting 159

B CURRICULUM VITAE 163

C ACKNOWLEDGEMENTS 165 


\section{INTRODUCTION}

Ranking of search results, network reliability, routing on the Internet and network layout are applications whose performance solely relies on algorithms. For example, most people use the Internet often to search, perhaps every day. Such search engines make the task of finding a website tractable, browsing through the whole Internet would take too much time. There are many keywords that thousands, perhaps millions of people search for, therefore making a good ranking of the search results for such a popular keyword has a huge impact. It is unlikely that any human can do this task quickly: there are billions pages of information on the Internet! This task is typically done by algorithms on computers. This is just one example where algorithms are used in our daily lives.

One particular area at the interface of mathematics and computer science where algorithms are heavily used is combinatorial optimization. Combinatorial optimization comprises problems where the goal is to find the "optimal" solution from a set of finite solutions. For each such problem there is an easy algorithm: enumerate all possible solutions and pick the one that is the optimum. In the example of ranking webpages, enumerating all different orderings would take more time than a full lifespan, even if there are only a hundred different webpages. I would have passed away before the result is computed.

In this thesis we design and analyze algorithms for several types of problems. Below we introduce the different topics in this thesis and then we briefly state the context in which we address these topics. The introductions to each chapter will contain more details on the definition of the individual problems and the corresponding related work.

\subsection{LATENCY COVERING PROBLEMS}

Everyone uses a search engine to find information on the Internet. Despite rapid advances in relating keywords to relevant pages, ordering the results remains difficult. People have different intentions when searching, 
even if they use the exact same keyword! The keyword itself can be ambiguous, people expect different results. For example, when searching for "Maastricht" both the municipality and the tourist information website are highly relevant. However, as a tourist the municipality information is less interesting but to a local it is relevant. Another difference is the quantity of results: for research one needs many results, but for navigational purposes a single result suffices.

To see why ordering results merely based on their significance to the majority can lead to bad orderings, consider the following example. What is the first thought about "lion"? Among other things, lion can refer to the name of the recent operating system for Apple computers; the species; a shop for beds; the airline company Lion Air. If we only consider the intent the majority has when searching for "lion", probably results for the operating system and the species dominate the first few pages of search results. However, by introducing a few search results on beds or flight information the minority will also be satisfied!

Each person, or group of people with similar intent, have a set of search results that they deem relevant and a threshold. The threshold is a number that indicates how many of such relevant search results they need to see before being satisfied. For example, when doing a literature search one needs perhaps 20 or 30 results while simply searching for the homepage of a company only requires a single result. Our goal is to find, for a given keyword, an ordering of relevant results that minimizes the average effort of all users.

The abstract problem is called Generalized Min Sum Set Cover and bears semblances to the well-known Set Cover problem. It is an example of a problem that can be called a "latency cover problem". Roughly speaking, a latency cover problem has multiple demands that need to be covered such that the average latency of all demands is minimized.

Submodular Ranking is another such latency cover problem. Submodular Ranking is a problem that extends Generalized Min Sum Set Cover. Simply put, we replace "sets" and "thresholds" by submodular functions. A function is called submodular if it posesses certain properties, informally we can say that submodularity models diminishing gains. In the example above, people were satisfied if the number of relevant search results exceeded a personal threshold. Replacing the set of relevant search results and the personal threshold by a submodular function allows interplay between different search results to have an effect. 
For example, consider searching for the key-word "lion". With submodular functions we can describe that the 10-th result about the mating habits of the African mountain lion does not contribute anything since the previous 9 already gave enough information on this topic. Another example is that each search result has different amounts of information. Putting a highly informative result first would be a good thing, but the novelty of its information diminishes after reading many other related results.

The previous problems looked at the situation where all the information is known a priori. What if the information is uncertain? In Stochastic Weighted Submodular we adaptively find an ordering of the search results that minimizes the total effort. Adaptively means that we give the ordering step-by-step and react to responses by the user as to gather more information what search results are relevant to the user. Secondly, we extend Submodular Cover to the case where each search result can have distinct lengths: the time it takes to read a search result differs per search result.

We introduce the Minimum Latency Submodular Cover problem, which is a very powerful problem that generalizes and relates quite some wellknown and notoriously hard problems such as Set Cover and Group Steiner Tree. It also generalizes all the other problems in Part one, except the stochastic setting. This in an interesting problem because it provides a unified view at all these problems.

Finally, Covering Steiner Tree is a generalization of Group Steiner Tree. The Latency Covering Steiner Tree problem is a natural version of Covering Steiner Tree, when the underlying metric is uniform it corresponds to Generalized Min Sum Set Cover.

\subsection{ROUTING ON THE INTERNET}

Communication is everything, by definition, on the Internet and the process of how messages are routed can be quite complex. The Internet is divided into autonomous systems and each autonomous system adheres to a clearly defined routing policy. An autonomous system can be owned by a single Internet service provider or by a collaboration between several network operators.

The Border Gateway Protocol (BGP) serves as the main routing protocol of the Internet and ensures network reachability among autonomous systems. When traffic is forwarded between the many autonomous systems 
on the Internet according to that protocol, each autonomous system selfishly routes the traffic inside its own network according to some internal protocol that supports the local objectives of the autonomous system.

We consider possibilities of achieving higher global performance in such systems while maintaining the objectives and costs of the individual autonomous systems. In particular, we consider how path trading, i.e. deviations from routing the traffic using individually optimal protocols, can lead to a better global performance.

\subsection{CONNECTIVITY IN GRAPHS}

When designing or evaluating networks there are many properties that are desirable or unwanted. For a network that should be robust, one can ask: how much damage can be done to the network but it still 'survives'. A less destructive motivation is from chip design. In the design of a chip a large number of components needs to be connected by wires, but preferably we have many parts that are only connected through a few hubs to make production easier. Depending on the precise definition, there are many beautiful mathematical graph connectivity problems. One such problem is k-Way Vertex Cut: the problem of finding a graph separator of a given size that decomposes the graph into the maximum number of components. That is, we are interested in knowing if after the removal of a given number of vertices the graph is not split into too many disconnected components.

\subsection{COMPUTATIONAL COMPLEXITY}

Designing efficient algorithms or proving that such algorithms do not exist for the problems introduced above is the goal of this thesis. In this section we explain what an "efficient" algorithm is, and how this can be leveraged to prove that a problem does not admit efficient algorithms.

The Cobham-Edmonds thesis states that an algorithm is efficient if its running time is guaranteed to be polynomial in the input length, no matter the input. Notice that the running time is guaranteed to be polynomial in the input length, regardless of the specific input. This type of analysis of the running time is called worst-case analysis. The property that an algorithm has worst-case polynomial running time is a very strong: no 
matter the input the algorithm finds the (optimal) solution in polynomial time. This model is very useful because we do not know what the input to the algorithm will be. Almost certainly we cannot predict what inputs our algorithm will get, and we certainly cannot analyze every different input separately. So, even without looking at each possible input separately we can guarantee the running time and optimality for each input! Besides this model there is also average-case analysis, in which it is assumed that the instance is generated according to some random process and smoothed analysis which is the middle ground between the two models.

The meaning of "efficient" is subjective and it is hard to come up with a clear, rigorous description which can also be applied to the analysis of algorithms. This is why the Cobham-Edmonds thesis is important, it precisely defines "efficient". While a polynomial-time algorithm is not always efficient in practice, more often than not there is an efficient algorithm if there is a polynomial-time algorithm and the reverse also holds. This motivates the rigorous mathematical analysis and design of algorithms. From this point on, when we say that an algorithm is efficient we mean that the algorithm runs in polynomial time.

When there exists an algorithm that finds the optimal solution in polynomial time for a problem, we say that the problem is polynomial-time solvable. There are problems that are not polynomial-time solvable, for example determining the winning move in generalized chess on an $n \times n$ board. More interestingly, there are problems of which it is not known if they are polynomial-time solvable or not. There are problems that are called NP-complete: every problem in the class NP can be reduced to those problems in polynomial time. If any NP-complete problem can be solved in polynomial time, then all problems in NP can be solved in polynomial time. There is a strong belief that among these there exist problems that cannot be solved in polynomial time.

The question whether $\mathrm{P}=\mathrm{NP}$ is one of the most important questions in mathematics and computer science. It is one of the seven Millennium Prize Problems selected by the Gray Mathematics Institute. The resolution of this question has far ranging consequences in for example cryptography. However, the importance of the mere fact whether $\mathrm{P}=\mathrm{NP}$ or $\mathrm{P} \neq \mathrm{NP}$ pales, in my opinion, to the possible treasure trove of deeper insights and fundamental understanding of what efficient computation entails and its relation to our surroundings that such a proof would provide.

There are several ways around the fact that a problem is NP-hard and therefore not polynomial-time solvable. We can trade efficiency for optimal- 
ity or we spend more than polynomial time. The idea of approximation is very old and intuitive: we are satisfied with a polynomial time algorithm that returns a very good, but perhaps not the optimal, solution. Such algorithms are called approximation algorithms. For a minimization problem, we say that an algorithm is a c-approximation if the algorithm always returns a solution with value ALG in polynomial time such that

$$
\mathrm{OPT} \leqslant \mathrm{ALG} \leqslant \mathrm{c} \cdot \mathrm{OPT},
$$

where OPT is the value of the optimal solution. Throughout this thesis, when talking about approximation algorithms we mean polynomial-time approximation algorithms.

As alluded to earlier, there is another type of analysis, namely smoothed analysis. This framework bridges a gap between practice and theory. In some cases it explains behavior in practice better than the classical theory, while still remaining mathematically rigorous. Analogously to the notion of polynomial-time solvable we now have the notion of smoothed polynomialtime solvable.

Smoothed analysis represents the middle ground between average case analysis and worst-case analysis. In worst-case analysis we say that the running time of an algorithm for instances of length $n$ is the supremum over all possible instances of length $n$. That is, if the algorithm has worst-case running time $t(n)$ than no matter what data is the input, we are guaranteed that the algorithm will take at most $t(n)$ time. This model is called adversial: imagine that there is an evil adversary whose only concern in life is to create inputs to make your algorithm slower. On the other hand, averagecase analysis measures the average running time over all instances. Since we cannot enumerate all instances, we assume that all instances are generated according to some rigorous random process. This begs the question whether "real life" inputs are random, which is also not realistic to assume.

Worst-case analysis could be viewed as pessimistic, and average-case analysis as optimistic. The question arises whether "real" data is adversial or random? In smoothed analysis the input is adversial but there is a slight random perturbation of the input. In this framework one can model that in practice there is inherent noise in the data, small perturbations caused by imprecise measurement for example. As a thought example, try to indicate how much an ordinary pencil is worth. Is it 50 cents, 1 Euro? Even if we would agree on 50 cents, why didn't you say 49.9 cents? Another thought experiment is to think about how data is collected. Suppose you are tasked with measuring the height of all daffodils in a garden with a ruler. Very 
likely the measurements will not be completely precise if only because of rounding the measurements to millimeters.

A famous example is the simplex algorithm. The simplex algorithm finds the optimal solution in linear programming, but there are (artificially) constructed instances for which it takes exponential time. However, the algorithm takes smoothed polynomial time! While there are polynomial time algorithms known for linear programming, the simplex method is quite simple and works well in practice. Therefore, with the framework of smoothed analysis we can unify practice and theory.

\subsection{THESIS OVERVIEW}

In this thesis we will discuss problems from the field of combinatorial optimization and we will analyze algorithms in the above mentioned frameworks.

We can summarize the goal of this thesis as follows.

\section{The goal of this thesis is to study latency cover and network optimization problems from the perspective of approximation algorithms and smoothed analysis.}

This thesis consists of eight chapters that, apart from the introduction in this chapter and preliminaries in Chapter 2, are split into two parts.

PART I The first part is on problems arising from search result ranking, broadcast scheduling and connectivity problems in chip design. These problems can be categorized as latency cover problems. Chapter 3 is an introductionary chapter to these latency cover problems, and elucidates their relationship and places them in historical context. Chapters 4 is on Generalized Min Sum Set Cover and Preemptive Generalized Min Sum Set Cover and we obtain good approximation algorithms. We do this by formulating a novel integer linear program, and rounding the relaxation to an integral solution in two separate steps. The next chapter, Chapter 5, provides an easier proof for an approximation algorithm for Submodular Ranking, which is leveraged to obtain good approximation algorithms for a vast generalization called Minimum Latency Submodular Cover and a stochastic version Weighted Stochastic Submodular Ranking. This part is concluded by Chapter 6 in which a new linear program is formulated for Covering Steiner Tree (an 
extension of Group Steiner Tree) which leads to a cleaner approximation algorithm for this problem and gives a good approximation algorithm for Latency Covering Steiner Tree.

PART II The second part contains two chapters about two network optimization problems. In Chapter 7 we explore the computational complexity of Path Trading, and we find a simple yet very fast algorithm that enumerates all Pareto-optimal solutions and has smoothed polynomial running time. We confirm the theory with experiments that show that practical performance is even faster than expected. Chapter 8 concerns the k-Way Vertex Cut problem for which we design an efficient polynomial time approximation scheme for planar graphs with techniques akin to the technique introduced by Baker. Further, we explore the complexity of this problem in several natural graph classes such as interval graphs, split graphs and graphs of bounded treewidth.

\subsection{PUBLICATIONS UNDERLYING THIS THESIS}

- S. Im, M. Sviridenko and R. van der Zwaan, "Preemptive and NonPreemptive Generalized Min Sum Set Cover" in International Symposium on Theoretical Aspects of Computer Science (STACS) 2012. Pages $465-476$.

- S. Im, V. Nagarajan and R. van der Zwaan, "Minimum Latency Submodular Cover" in International Colloquium on Automata, Languages and Programming (ICALP) 2012.

- A. Berger, H. Röglin and R. van der Zwaan, "Path Trading: Fast Algorithms, Smoothed Analysis, and Hardness Results" in Experimental Algorithms (SEA) 2011. Lecture Notes in Computer Science 6630:43-53, 2011.

- A. Berger, A. Grigoriev and R. van der Zwaan, “How to Cut a Graph into Many Pieces" in Theory and Applications of Models of Computation (TAMC) 2011. Lecture Notes in Computer Science 6648:184-194, 2011. 


\section{PRELIMINARIES}

These preliminaries explain basic notation and certain tools that are used in multiple chapters. However, some concepts are only explained in the chapter themselves, for example treewidth, since only Chapter 8 uses it.

\subsection{BASIC NOTATION}

SETS AND FUNCTIONS Sets are usually denoted by a capital letter and sets of sets by a calligraphic letter. We adopt standard notation for the sets of numbers: $\mathbb{N}, \mathbb{Q}, \mathbb{R}$ for the natural numbers, rational numbers and real numbers, respectively. The subscript $\mathbb{N}_{+}$indicates that the set only contains positive numbers. We let $[n]=\{1, \ldots, n\}$.

Given a function $f: E \rightarrow D$ then, depending on context, we denote for convencience $f(S)=\sum_{e \in S} f(e)$ or $f(S)=\bigcup_{e \in S} f(e)$. For a function $f: E \rightarrow D$, let $f^{-1}: D \rightarrow E$ denote its inverse.

The power set of a set $\mathrm{E}$ is denoted by $2^{\mathrm{E}}$, the power set is the set of all subsets of $E$. A w-partition of a set $E$ is a partition of $E$ into $w$ (possibly empty) subsets of $\mathrm{E}$, and we denote the set of all $w$-partitions of $\mathrm{E}$ by $w^{\mathrm{E}}$.

For a predicate $\phi$ let $\mathbf{1}[\phi]$ be its indicator function, that is $\mathbf{1}[\phi]=1$ if and only if $\phi$ is true and zero otherwise.

GRAPHS An undirected graph consists of a set $V$ of vertices and a set $E$ of unordered pairs of distinct vertices. An edge is denoted as $\{u, v\}$ where $u, v \in \mathrm{V}$. The open neighbourhood $\mathrm{N}(v)$ of a vertex $v$ are all vertices $u$ such that $\{u, v\} \in E$, and the closed neighbourhood $N[v]=N(v) \cup\{v\}$.

ASYMPTOTIC NOTATION For a function $f$ the set $O(f(n))$ contains all functions $g(n)$ such that there are constants $n_{0}$ and $c$ such that $g(n) \leqslant c \cdot f(n)$ for all $n \geqslant n_{0}$. Likewise, for a function $f$ the set $\Omega(f(n))$ contains all functions $g(n)$ such that there are constants $n_{0}$ and $c$ such that $g(n) \geqslant c \cdot f(n)$ for all $n \geqslant n_{0} . \Theta(f(n))$ is the set of functions $g(n)$ such that $g(n) \in O(f(n))$ and $g(n) \in \Omega(f(n))$. O* $(f(n))$ is the set of functions $g(n)$ such that there 
is a polynomial $p(n)$ and a constant $n_{0}$ such that $g(n) \leqslant p(n) \cdot f(n)$ for all $n \geqslant n_{0}$. The function $g(n)$ is $o(f(n))$ as $n \rightarrow \infty$ if for every positive constant $\epsilon$ there is an $n_{0}$ such that $|g(n)| \leqslant \epsilon|f(n)|$ for all $n \geqslant n_{0}$.

\subsection{COMPUTATIONAL COMPLEXITY}

The class $\mathrm{P}$ contains all decision problems that can be solved by a deterministic algorithm in time bounded by a polynomial in the input length. The class NP are all decision problems solvable by a non-deterministic algorithm in time bounded by a polynomial in the input length. Whether $\mathrm{P}=\mathrm{NP}$ is open, although the consensus seems to be that $\mathrm{P} \neq \mathrm{NP}$. There exist problems in NP, that each contain any other problem in NP as a special case. We call these general problems NP-complete, they are the hardest problems in NP. A consequence of $P \neq N P$ is that there are no deterministic polynomial time algorithms for NP-complete problems. The class RP are problems that can be solved by a randomized algorithm in polynomial time with one-sided error at most 1/2, and the class BPP are problems that can be solved by a randomized polynomial time algorithm with twosided error at most 2/3. Clearly, $\mathrm{P} \subseteq \mathrm{RP}$ and $\mathrm{P} \subseteq \mathrm{BPP}$, and the commonly believed conjecture is that $\mathrm{P}=\mathrm{RP}=\mathrm{BPP}$.

The class BPTIME $(f(n))$ contains all problems that can be solved in $f(n)$ time with two-sided error of at most $2 / 3$. A widely held belief is that NP does not have randomized sub-exponential time algorithms, and is formalized in the following conjecture.

Conjecture 2.1 (See Khot [70]). The class NP does not have randomized subexponential time algorithms, e.g.

$$
\mathrm{NP} \nsubseteq \bigcap_{\epsilon>0} \operatorname{BPTIME}\left(2^{n^{\epsilon}}\right)
$$

A related conjecture is the exponential time hypothesis. A k-CNF-formula over $n$ variables $v_{1}, . ., v_{n}$ is a logical formula that is a conjunction of clauses $\phi=C_{1} \wedge \ldots \wedge C_{m}$ where every clause is a disjunction of literals $C_{i}=$ $l_{i, 1} \vee \ldots \vee l_{i, \ell}$ where $\ell \leqslant k$ and every literal is either $v_{j}$ or $\neg v_{j}$ for some $1 \leqslant j \leqslant n$. The formula $\phi$ is satisfied by an assignment of true and false to the literals such that substituting the variables by these values makes $\phi$ true. The problem k-CNF-Sat is to find, given a k-CNF-formula, an assign- 
ment of variables that makes the formula true or determine that this is not possible.

Conjecture 2.2 (Exponential Time Hypothesis, [65]). Let $s_{k}$ be the infimum of the real numbers $\delta$ such that there is an algorithm with $\mathrm{O}^{*}\left(2^{\text {dn }}\right)$ time that solves $\mathrm{k}-\mathrm{CNF}$-Sat. Then for every $\mathrm{k}, \mathrm{s}_{\mathrm{k}}>0$.

Another conjecture is that NP does not have quasi-polynomial time algorithms. An algorithm algorithm with running time $\mathrm{O}\left(2^{(\mathrm{O}(\log n))^{\mathrm{O}(1)}}\right)$ has quasi-polynomial running time. Notice that if any problem that is $\mathrm{NP}$ - complete is shown to have an algorithm with quasi-polynomial time then all problems in NP have quasi-polynomial time algorithms. This is in contrast to sub-exponential time algorithms. Given any problem in NP there is a polynomial time transformation from that problem to any other $\mathrm{NP}$ - complete problem. In other words, given an instance of size $n$ we can transform this to an instance of size $\mathrm{n}^{\mathrm{O}(1)}$ of the problem for which we have a quasi-polynomial time algorithm.

The class $\operatorname{DTIME}(f(n))$ contains all problems that can be solved deterministically in $f(n)$ time.

Conjecture 2.3 (See [40]). The class NP does not have quasi-polynomial time algorithms, e.g.

$$
N P \nsubseteq \bigcup_{c \in \mathbb{N}} \operatorname{DTIME}\left(2^{(\log n)^{c}}\right)
$$

\subsubsection{Fixed-parameter tractability}

In the definition of $\mathrm{P}$ and NP the length of the input was the defining parameter in which we measured the running time. However, there could be more parameters involved. As an example we take Vertex Cover and Dominating Set. Both problems get as input a graph $G=(V, E)$ and an integer $k$. Vertex Cover asks for a subset $S \subset \mathrm{V}$ of cardinality at most $k$ such that every edge $e \in E$ is incident to at least one vertex in S. In Dominating Set the goal is to find a subset $S \subset V$ of cardinality at most $k$ such that every vertex $v \in \mathrm{V} \backslash \mathrm{S}$ is adjacent to a vertex in $\mathrm{S}$. Both problems are NP-hard, but when $k$ is fixed (not part of the input) there is a simple polynomial time algorithm: enumerate all subsets of $\mathrm{V}$ of cardinality at most $\mathrm{k}$ and test whether it is a valid solution (which can be done in polynomial time). However, if $|\mathrm{V}|$ is large an $\mathrm{O}\left(|\mathrm{V}|^{\mathrm{k}}\right)$ time algorithm is not desirable. 
An algorithm is called fixed-parameter tractable with regard to a parameter $k$ if the running time is $f(k) \cdot n^{O}(1)$, where $f$ is some function that only depends on $k$. The idea is that there are many instances for which $k$ is reasonably small but the length of the input is incredibly large. There is a hierarchy of problems that are conjectured to not have fixed-parameter tractable algorithms: the W-hierarchy by Downey and Fellows [36]:

$$
\mathrm{FPT} \subseteq \mathrm{W}[1] \subseteq \ldots \subseteq \mathrm{W}[\text { poly }],
$$

where is FPT is the class of problems that are fixed-parameter tractable.

Analoguously to NP and NP-hardness a problem can be W[1]-hard: such a problem is at least as hard as any problem in the class $W[1]$. It is conjectured that FPT $\neq W[1]$, which means that for problems that are $W[1]$-hard there are no fixed-parameter tractable algorithms. To return to our example, despite their apparent similarity Vertex Cover is in FPT and Dominating Set is $W[2]$-hard [36].

\subsection{APPROXIMATION ALGORITHMS}

There are problems for which no polynomial time algorithm is known, and that are in fact NP-hard. For these problems a polynomial time algorithm that finds the optimal solution is unlikely. This justifies approximation algorithms, basically the goal becomes to find a good approximate solution in polynomial time. Approximation algorithms are an active topic of research and is a vibrant field.

In this thesis when we talk about approximation algorithms we also want that their running time is polynomial, unless explicitly stated otherwise. This is not always the case in the literature: there are approximation algorithms that use super-polynomial, even exponential, time.

For a maximization problem an algorithm is a c-approximation if the algorithm always returns a solution with value ALG in polynomial time such that

$$
\mathrm{OPT} \geqslant \mathrm{ALG} \geqslant \mathrm{OPT} / \mathrm{c},
$$

where OPT is the value of the optimal solution.

For a minimization problem an algorithm is a c-approximation if the algorithm always returns a solution with value ALG in polynomial time such that

$$
\mathrm{OPT} \leqslant \mathrm{ALG} \leqslant \mathrm{c} \cdot \mathrm{OP}
$$


where OPT is the value of the optimal solution.

An Polynomial Time Approximation Scheme (PTAS) (for a maximization problem) is a polynomial-time approximation algorithm that for any fixed $\epsilon>0$ returns a solution of value at least $(1-\epsilon)$ times the optimum in polynomial time.

An Efficient Polynomial Time Approximation Scheme (EPTAS) (for a maximization problem) is a polynomial-time approximation algorithm that for any given $\epsilon>0$ returns a solution of value at least $(1-\epsilon)$ times the optimum in time poly $(n) f(\epsilon)$, where poly $(n)$ only depends on $n$ and not on $\epsilon$, and $f(\cdot)$ is some function only depending on $\epsilon$.

\subsection{UNIQUE GAMES CONJECTURE}

The Unique Games Conjecture, which will be formally stated below, has been very influential in the search for lower bounds on approximation ratios. For example, for the Vertex Cover problem there is a very simple 2-approximation, independently discovered by Gavril and Yannakakis. Furthermore, Dinur and Safra showed that Vertex Cover cannot be approximated within a factor smaller than 1.3606 unless $P=N P$ [37]. Using the Unique Games Conjecture, which is a stronger conjecture than $\mathrm{P} \neq \mathrm{NP}$, Khot and Regev showed in that Vertex Cover cannot be approximated within a factor smaller than 2 [72]. Another well known example is the Maximum Cut problem, which can be approximated within a factor 0.87856 by an algorithm by Goemans and Williamson [47]. Again, the lower bound modulo $\mathrm{P}=\mathrm{NP}$ is a low constant namely $16 / 17$ by Håstad and Trevisan, Sorkin, Sudan and Williamson [62, 104], but assuming the Unique Games Conjecture this approximation algorithm is the best possible [71].

We wish to stress that there seems to be no consensus regarding the truth of the conjecture. The fact that the Unique Games Conjecture provided matching lower bounds to the known upper bounds for some well-studied problems is quite impressive. However, this can also be explained that this is the limit of currently known techniques rather than an inherent barrier, as $P$ versus NP is believed to be. Indeed, there seems to be a connection between Unique Games Conjecture and the approximability of semidefinite programming relaxations which is arguably among the most powerful algorithmic tools at our disposal. Raghavendra showed that there is a class of problems for which there is a semidefinite programming relaxation such 
that, assuming the Unique Games Conjecture, no other polynomial time algorithm can provide a better approximation than said relaxation [88].

On the negative side, a subexponential time algorithm was discovered recently for the Unique Games Problem [3], which is conjectured to be NPhard in the Unique Games Conjecture.

For more background information, we refer to two excellent surveys of Trevisan and Khot on the Unique Games Conjecture [69, 103].

An instance $\mathcal{L}\left(\mathrm{G}(\mathrm{V}, \mathrm{W}, \mathrm{E}),[\mathrm{n}],\{\sigma(v, w)\}_{\{v, w\} \in \mathrm{E}}\right)$ of Unique Games consists of a regular bipartite graph $\mathrm{G}(\mathrm{V}, \mathrm{W}, \mathrm{E})$ and a set $[n]$ of labels. For each edge $\{v, w\} \in E$ there is a constraint specified by a permutation $\sigma(v, w):[n] \rightarrow[n]$. The goal is to find a labeling $\ell: V \cup W \rightarrow[n]$ of the vertices such that as many edges as possible are satisfied, where an edge $e=\{v, w\}$ is said to be satisfied if $\ell(v)=\sigma(v, w)(\ell(w))$.

Given a Unique Games instance

$$
\mathcal{L}\left(\mathrm{G}(\mathrm{V}, \mathrm{W}, \mathrm{E}),[\mathrm{n}],\left\{\sigma(v, w)_{\{v, w\} \in \mathrm{E}}\right)\right.
$$

let $\mathrm{OPT}(\mathcal{L})$ denote the maximum fraction of simultaneously satisfied edges of $\mathcal{L}$ by any labeling, i.e.

$$
\mathrm{OPT}(\mathcal{L}):=\frac{1}{|\mathrm{E}|} \max _{\ell: \mathrm{V} \cup W \rightarrow[n]} \mid\{e \in E: \ell \text { satisfies } e\} \mid .
$$

Conjecture 2.4 (Unique Games Conjecture, see Bansal and Khot [9]). For any constants $\zeta, \gamma>0$, there is a sufficiently large constant $n=n(\zeta, \gamma)$ such that, for Unique Games instances $\mathcal{L}$ with label set $[n]$ it is NP-hard to distinguish between

- $\mathrm{OPT}(\mathcal{L}) \geqslant 1-\zeta$,

- $\mathrm{OPT}(\mathcal{L}) \leqslant \gamma$

\subsection{LINEAR PROGRAMMING}

In this thesis we make extensive use of linear programming, and this section introduces the necessary notions.

A subset $C$ of $\mathbb{R}^{n}$ is convex if $\lambda x+(1-\lambda) y$ belongs to $C$ for all $x, y \in C$ and each $\lambda$ with $0 \leqslant \lambda \leqslant 1$. The convex hull conv.hull $(X)$ of a set $X \subseteq \mathbb{R}^{n}$ is the smallest convex set containing $X$. 
A subset $\mathrm{P}$ of $\mathbb{R}^{\mathrm{n}}$ is a polyhedron if there exist an $\mathrm{m} \times \mathrm{n}$ matrix $A$ and a vector $b \in \mathbb{R}^{m}$ for some $m \geqslant 0$ such that

$$
P=\{x \mid A x \leqslant b\} .
$$

A subset $\mathrm{P}$ of $\mathbb{R}^{\mathrm{n}}$ is a polytope if there is a finite set $\mathrm{X} \subseteq \mathbb{R}^{\mathrm{n}}$ such that

$$
\mathrm{P}=\operatorname{conv} \cdot \operatorname{hull}(\mathrm{X}) \text {. }
$$

Linear programming is the problem of minimizing or maximizing a linear function over a polyhedron. For example

$$
\min \left\{c^{\top} x \mid x \geqslant 0, A x \geqslant b\right\}
$$

We refer to entries of the vector $x$ as the variables and to the rows in $A x \geqslant b$ as constraints.

Theorem 2.1 (Linear programming duality). Let $\mathrm{A}$ be a matrix and $\mathrm{b}$ and $\mathrm{c}$ be vectors. Then

$$
\max \left\{c^{\top} x \mid A x \leqslant b\right\}=\min \left\{y^{\top} b \mid y \geqslant 0, y^{\top} A=c^{\top}\right\}
$$

if at least one of these optima is finite.

For any polytope $\mathrm{P}$ in $\mathbb{R}^{n}$, a point $\mathrm{p} \in \mathrm{P}$ is an extreme point (or vertex) if there is no vector $x \in \mathbb{R}^{n}$ such that both $p+x$ and $p-x$ are in $P$. By definition, a polytope is the convex hull of its extreme points, so we have that for any polytope $P$ with extreme points $E$ we have $P=\operatorname{conv} \cdot h u l l(E)$. For a linear program that has a finite optimum, this optimum is attained at an extreme point of the polytope/polyhedra.

For a polytope $P=\{x \mid A x \leqslant b\}$ and a point $x$ we say that a constraint $i$ is tight at $x$ if $A_{i} x=b_{i}$.

Theorem 2.2 (Rank lemma). Let $P=\{x \mid A x \leqslant b, x \geqslant 0\}$ and $x$ be an extreme point solution such that $x_{i}>0$ for each $i$. Then the number of variables is equal to the number of linearly independent constraints of $A$, i.e. the rank of $A$.

A polyhedron $\mathrm{P}$ is an integer polyhedron if it is the convex hull of the integer vectors contained in $\mathrm{P}$. Equivalently, a polytope $\mathrm{P}$ is integer if and only if every extreme point of $P$ is integer. 


\subsubsection{Solving linear programs}

Perhaps the best known algorithm to solve a linear program is the simplex method by George Dantzig in 1951, however there are (artificially constructed) instances where the running time is exponential. There are several algorithms that solve linear programs in polynomial time, the first was found by Khachiyan in 1979 who used the 'ellipsoid method' which is practically infeasible but of theoretical importance. Karmarkar in 1984 showed that there are interior point methods that have polynomial running time and have practical implementations. Despite that the ellipsoid method is not practically feasible, typically with a technique called "column generation" it is possible to solve many such problems practically.

Theorem 2.3 (Linear programming). Given a linear program with $\mathrm{n}$ variables and $\mathrm{m}$ constraints. Then, there is an algorithm that solves the linear program in time polynomial in $\mathrm{n}$ and $\mathrm{m}$.

The ellipsoid algorithm has an advantage compared to interior point methods. The constraints need not to be explicitly listed beforehand, but rather be generated when needed. Below we state this formally.

The ellipsoid algorithm takes as input a convex set, and returns a point from the set provided it is nonempty and returns "empty" otherwise. Since solving a linear program reduces to testing if a linear program is feasible, this algorithm can also be used to solve linear programs as well.

We assume the convex set $\mathrm{K}$ is defined by the intersection of half-spaces, or equivalently linear constraints. Otherwise, for general convex sets, the solution might be irrational and it becomes unclear how to output the precise answer in polynomial time.

Theorem 2.4 (Ellipsoid algorithm). Suppose there is a polynomial-time algorithm for a convex set $\mathrm{K}$, when given as input $\mathrm{s} \in \mathbb{Q}^{\mathrm{n}}$, either returns " $\mathrm{s} \in \mathrm{K}$ " or $\mathrm{a} \in \mathbb{Q}^{\mathrm{n}}$ such that $\mathrm{a}^{\top} \mathrm{x}<\mathrm{a}^{\top} \mathrm{s}$ for all $\mathrm{x} \in \mathrm{K}$. Then, the ellipsoid algorithm runs in polynomial time and returns a point from $\mathrm{K}$ if $\mathrm{K}$ is nonempty or returns that $\mathrm{K}$ is empty.

\subsection{SUBMODULARITY}

Let $\mathrm{V}$ denote a ground set of elements. A function $f: 2^{V} \rightarrow \mathbb{R}$ is said to be submodular if it satisfies the property that for any $A, B \subseteq V, f(A)+f(B) \geqslant$ 
$f(A \cup B)+f(A \cap B)$. It is well-known that this is equivalent to the following property: For any $A \subseteq B$ and any $e \in V \backslash B, f(A \cup\{e\})-f(A) \geqslant f(B \cup\{e\})-$ $f(B)$.

Many interesting objectives can be modeled as submodular functions. For example, in economics, the latter property of a submodular function can be viewed essentially as the law of diminishing marginal utility. Also submodular functions appear often in combinatorial optimization. Such examples include set unions, cut functions, matroid rank functions etc. We refer the reader to [92] for an extensive overview of submodular functions and their applications.

From the definition of submodularity, the following useful inequality can be derived.

Proposition 2.5. For any $\mathrm{A}, \mathrm{B} \subseteq \mathrm{V}$ and submodular function $\mathrm{f}$, it follows that

$$
\sum_{e \in A}(f(B \cup\{e\})-f(B)) \geqslant f(B \cup A)-f(B)
$$

A submodular function $f$ is said to be monotone if for any $A \subseteq B, f(A) \leqslant$ $f(B)$.

Since function $f$ is defined over all possible subsets of $V$, it is a standard assumption that a value oracle for $f$ is available. The oracle takes as input a subset $S \subseteq V$ and returns the function value $f(S)$ in constant time.

Any non-negative linear combination of submodular functions is also a submodular function. Formally, for submodular functions $f_{1}, \ldots, f_{k}$ and nonnegative numbers $\alpha_{1}, \ldots, \alpha_{k}$ then $g(S)=\sum_{i=1}^{k} \alpha_{i} f_{i}(S)$ is also submodular.

\subsection{MATROIDS}

A pair $(S, \mathcal{J})$ is called a matroid if $S$ is a finite set and $\mathcal{J}$ is a nonempty collection of subsets of $S$ satisfying:

1. if $\mathrm{I} \in \mathcal{J}$ and $\mathrm{J} \subseteq \mathrm{I}$, then $\mathrm{J} \in \mathcal{J}$,

2. if $\mathrm{I}, \mathrm{J} \in \mathcal{J}$ and $|\mathrm{I}|<|\mathrm{J}|$, then $\mathrm{I}+z \in \mathcal{J}$ for some $z \in \mathrm{J} \backslash \mathrm{I}$.

The set $\mathcal{J}$ is also known as the set of independent sets of $M$.

The independent set polytope $\mathrm{P}$ of a matroid $M=(S, J)$ is the convex hull of the incidence vectors of the independent sets of $M$. So, $P$ is a polytope in $\mathbb{R}^{S}$. Then, the extreme points of $\mathrm{P}$ are integer. 
A partition matroid is a matroid where $S$ is partitioned into disjoint sets $\mathrm{S}_{1}, \ldots, \mathrm{S}_{\mathrm{k}}$ and

$$
\mathcal{J}=\left\{X \subseteq S|| X \cap S_{i} \mid \leqslant c_{i} \text { for all } i=1, \ldots, k\right\},
$$

for some parameters $c_{1}, \ldots, c_{k}$.

A family $\mathcal{F}$ of sets is laminar if for all $X, Y \in \mathcal{F}$ either (i) $X \subseteq Y$, or (ii) $\mathrm{Y} \subseteq \mathrm{X}$, or (iii) $\mathrm{X} \cap \mathrm{Y}=\emptyset$. A laminar matroid is a matroid if there is a laminar family $\mathcal{F}$ of subsets of $S$ and an integer $c(A)$ for every $A \in \mathcal{F}$ such that

$$
\mathcal{J}=\{X \subseteq S|| X \cap A \mid \leqslant c(A) \text { for all } A \in \mathcal{F}\}
$$

Theorem 2.6 (Edmonds 1970, see [92]). Let $M_{1}=\left(S, J_{2}\right)$ and $M_{2}=\left(S, J_{2}\right)$ be matroids and $\mathrm{P}_{1}$ and $\mathrm{P}_{2}$ their independent set polytopes, respectively. Then, $\mathrm{P}_{1} \cap \mathrm{P}_{2}$ is the convex hull of common independent sets $\mathcal{J}_{1} \cap \mathcal{J}_{2}$. Further, $\mathrm{P}_{1} \cap \mathrm{P}_{2}$ is an integral polytope. 
Part I

MINIMUM LATENCY COVERING 



\section{LATENCY COVERING: INTRODUCTION}

The common denominator of the problems discussed in the following three chapters is to find an ordering of a set of elements that simultaneously achieves a good performance with relation to several objective functions. Even though most of these problems were motivated by different applications, they are intimately related. Applications range from broadcast scheduling to survivable network design and ranking search results to VLSI design. Beyond the applicability, the theoretical aspects and implications are similarly fascinating.

This chapter is an introduction and literature overview for the problems discussed in Chapters 4, 5 and 6. The contents are summarized in Figure 1: it shows the relations between the various problems discussed in the following chapters. For each of these problems the definition, its relation to other problems and a short overview of relevant literature is given.

\section{Set Cover (SC) / Hitting Set}

For the Hitting Set problem we are given elements $[n]$ and sets $S_{1}, \ldots, S_{m} \subseteq$ $\{1, \ldots, n\}=[n]$. The goal is to find a minimum cardinality subset $T \subseteq[n]$ such that every set $S_{i}$ is "hit" i.e. for every set $S_{i}$ there is an element $e$ such that $e \in\left(S_{i} \cap T\right)$. This is equivalent to the Set Cover problem, where we are given sets $S_{1}, \ldots, S_{n}$ and elements $[\mathrm{m}]$ and the goal is to find the minimum cardinality set of sets $\mathcal{T} \subseteq\left\{S_{1}, \ldots, S_{n}\right\}$ that covers all elements. An element $e \in[\mathrm{m}]$ is said to be covered if there is a set $S \in \mathcal{T}$ with $e \in S$.

Set Cover is a classical problem and its study has spawned many techniques used in approximation algorithms. A simple greedy algorithm pick the set that covers the most uncovered elements - achieves an approximation of $\mathrm{H}(\mathrm{s})$ where $\mathrm{s}$ is the cardinality of the largest set and $\mathrm{H}(\mathrm{t})$ is the $t$-th harmonic number. In other words, the approximation ratio is

$$
\sum_{\mathfrak{i}=1}^{s} \frac{1}{\mathfrak{i}} \leqslant \ln s+1 \quad \text { where } s=\max \left\{\left|S_{1}\right|, \ldots,\left|S_{n}\right|\right\} .
$$




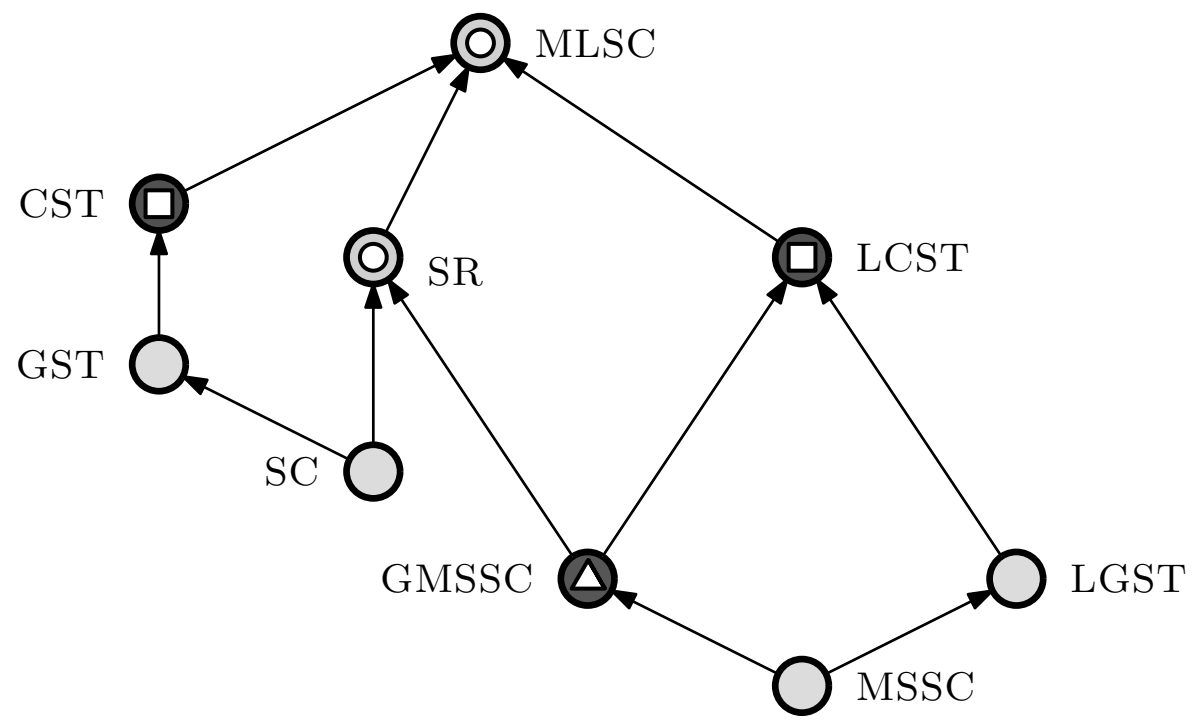

$\triangle$ Chapter 3 (() Chapter 4 (ㅁ) Chapter 5

Figure 1: The various problems and their relations. An arrow from $X$ to $Y$ means $X$ is a special case of $Y$. (Abbreviations are explained in the following paragraphs)

This simple algorithm is surprisingly the best possible under reasonable complexity assumptions. Feige proved that there is no $(1-o(1)) \cdot \ln n$ approximation unless NP has quasi-polynomial time algorithms [40] (See Conjecture 2.3). Raz and Safra proved a lower bound of $c \cdot \ln n$ where $c$ is a constant under the assumption that $\mathrm{P} \neq \mathrm{NP}[89]$. More recently, Alon, Moshkovitz and Safra improved the value of the constant c [2].

Min Sum Set Cover (MSSC)

The Min Sum Set Cover problem is a minimum latency version of the hitting set problem. We are given as input $n$ elements, $[n]$ and a collection of sets $\mathcal{S}=\left\{S_{1}, S_{2}, \ldots, S_{m}\right\}$ where each set $S_{i} \in 2^{[n]}$. The goal is to find a permutation of the elements such that the total sum of (or equivalently average) cover times of all sets is minimized. For simplicity, we will say that 
an element $e$ is covered at time slot $t$ or it has cover time $\operatorname{cov}(e)=t$ if it is placed in the $\mathrm{t}$-th position in the permutation. Equivalently, we may say that the element $e$ is scheduled at time $t$. The cover time $\operatorname{cov}\left(S_{i}\right)$ of a set $S_{i}$ is defined as $\min _{e \in S_{i}} \operatorname{cov}(e)$ and the goal is to minimize $\sum_{S_{i} \in \mathcal{S}} \operatorname{cov}\left(S_{i}\right)$.

For this problem, a simple greedy algorithm is known to achieve an approximation factor $4[10,41]$. The greedy algorithm iteratively picks the element that covers the most sets that are not yet covered. Also it is known that the problem cannot be approximated within a factor of $4-\epsilon$ for any $\epsilon>0$ unless $P=N P$ [41]. A closely related problem known as Min Sum Coloring was studied before in [10, 12] with applications in scheduling. Also the special case of the Min Sum Vertex Cover was used in [20] as a heuristic for speeding up a solver for semidefinite programs.

\section{Generalized Min Sum Set Cover (GMSSC)}

A generalization of the aforementioned problems was introduced by Azar, Gamzu and Yin [6] to provide a better framework for ranking web pages in response to queries that could have multiple intentions. This generalized problem was later named Generalized Min Sum Set Cover [8], and can be stated as follows. Every set $S_{i}$ has a requirement $\kappa\left(S_{i}\right) \in\left\{1,2, \ldots,\left|S_{i}\right|\right\}=$ $\left[\left|S_{i}\right|\right]$. For a permutation of the ground set we define $\operatorname{cov}(e)$ as before and $S_{i}$ is covered at time $t$ if $t$ is the earliest time such that $\mid\left\{e \in S_{i}: \operatorname{cov}(e) \leqslant\right.$ $t\} \mid \geqslant \kappa\left(S_{i}\right)$. Again, the goal is to find a permutation of the elements in [n] minimizing $\sum S_{i} \in \mathcal{S} \operatorname{cov}\left(S_{i}\right)$.

Azar et al. [6] give a modified greedy algorithm that has a performance guarantee of $\mathrm{O}\left(\ln \left(\max _{S_{i} \in \mathcal{S}} \kappa\left(S_{i}\right)\right)\right)$. The question whether there exists an $\mathrm{O}(1)$-approximation was answered affirmatively by Bansal, Gupta and $\mathrm{Kr}$ ishnaswamy [8]. In order to obtain an $\mathrm{O}(1)$-approximation, they used a time indexed linear program together with knapsack cover inequalities and gave a clever randomized rounding scheme. Very recently, their approximation ratio of 485 was improved by Skutella and Williamson to 28 via the same LP but a different rounding scheme [98].

The Min Latency Set Cover problem is a variant where the cover time is defined as the time where all elements in the set are covered e.g. $\operatorname{cov}\left(S_{i}\right)=$ $\max _{e \in S_{i}} \operatorname{cov}(e)$. This problem is in fact equivalent to the precedence-constrained scheduling problem on a single machine [106], for which various 2approximation algorithms are known $[57,27,80]$. It was shown that, assuming a variant of the Unique Games Conjecture, unless $\mathrm{P}=\mathrm{NP}$ there is 
no $2-\epsilon$ approximation for any $\epsilon>0$ [9] for the Min Latency Set Cover problem (See Conjecture 2.4).

CONTRIBUTIONS In this thesis we give the best known polynomial time approximation algorithm for this problem. We look at a relaxation of this problem, Preemptive Generalized Min Sum Set Cover and develop a polynomial time 2-approximation for this problem. Then, we show that given a solution to the Preemptive Generalized Min Sum Set Cover problem we can transform this into a solution to Generalized Min Sum Set Cover with only a 6.2-factor loss, which results in a 12.4-approximation to Generalized Min Sum Set Cover. Both algorithms are randomized.

\section{Submodular Ranking (SR)}

For Submodular Ranking we are given as input $\mathrm{n}$ elements $\mathrm{V}$ and monotone submodular functions $f_{1}, f_{2}, \ldots f_{m}: 2^{V} \rightarrow[0,1]$ with $f_{i}(V)=1, \forall i \in[m]$. The goal is to find a complete linear ordering of the elements that minimizes the total cover time of all functions. The cover time $\operatorname{cov}\left(f_{i}\right)$ of $f_{i}$ is defined as the smallest index $t$ such that the function $f_{i}$ has value $I$ on the first $t$ elements in the ordering. A technical parameter that we use to measure performance is $\epsilon$ which is defined to be the smallest non-zero marginal increase of any function $\left\{f_{i}\right\}_{i=1}^{m}$.

As shown in Azar and Gamzu [5], the Submodular Ranking problem contains set-cover as a special case (even when $m=1$ ). Azar and Gamzu give an efficient $\mathrm{O}\left(\log \epsilon^{-1}\right)$-approximation algorithm, whose analysis is involved.

CONTRIBUTIONS We give a substantially simpler proof of the approximation algorithm for Submodular Ranking by Azar and Gamzu, which will be used to obtain the results for Stochastic Weighted Submodular Cover and Minimum Latency Submodular Cover.

\section{Weighted Stochastic Submodular Ranking}

In Weighted Stochastic Submodular Ranking we are given a set V of stochastic elements (random variables), each having an independent distribution over a certain domain $\Delta$. The submodular functions are also defined on the ground set $\Delta$, i.e. $f_{1}, \ldots, f_{m}: 2^{\Delta} \rightarrow[0,1]$. In addition, each element 
$i \in V$ has a deterministic time $\ell_{i}$ to be scheduled. The realization (from $\Delta$ ) of any element is known immediately after scheduling it. The goal is to find an adaptive ordering of $\mathrm{V}$ that minimizes the total expected cover time. Since elements are stochastic, it is possible that a function is never covered: in such cases we just fix the cover time to be $\sum_{i \in V} \ell_{i}$ (which is the total duration of any schedule).

We will be concerned with adaptive algorithms. Such an algorithm is allowed to decide the next element to schedule based on the instantiations of the previously scheduled elements. This models the setting where the algorithm can benefit from user feedback.

The Adaptive Set Cover problem introduced in [46] is clearly a special case of Weighted Stochastic Submodular Ranking. Goemans and Vondrák [46] showed a large adaptivity gap for set cover, and a logarithmic approximation for a variant with multiplicities. A related problem in context of fast query evaluation was studied in Munagala et al. [83], where the authors gave a triple logarithmic approximation. This bound was improved to the optimal logarithmic ratio by Liu et al. [78]; this result was also applicable to adaptive set cover. Another related paper is by Golovin and Krause [50], where they defined a general property "adaptive submodularity" and showed nearly optimal approximation guarantees for several objectives (max coverage, min-cost cover and min sum cover). The most relevant result in [50] to the Weighted Stochastic Submodular Ranking problem is the 4-approximation for Stochastic Min Sum Set Cover. This approach required a fixed submodular function $f$ such that the objective is $\mathbb{E}\left[\sum_{t \geqslant 0} f(\bar{V})-f\left(\bar{\pi}_{t}\right)\right]$ where $\bar{\pi}_{t}$ is the realization of elements scheduled within time $t$ and $\bar{V}$ denotes the realization of all elements. However, as mentioned earlier this is not the case even for generalized min-sum set cover with requirements two.

CONTRIBUTIONS We extend our analysis of Submodular Ranking to the weighted and stochastic case. This results in an adaptive algorithm that generalizes previous results and matches the performance guarantees of the algorithms tailored to the more specific problems. 
Minimum Latency Submodular Cover (MLSC)

Minimum Latency Submodular Cover is the metric version of Submodular Ranking [5], and the latency covering Steiner tree problem (LCST) which extends generalized min-sum set cover [6,8].

We let $\mathrm{V}$ denote the ground set of elements/vertices and $\mathrm{d}:\left(\begin{array}{l}\mathrm{V} \\ 2\end{array}\right) \rightarrow \mathbb{R}_{+}$ the distance function. We assume that $d$ is symmetric and satisfies the triangle inequality. In addition there is a specified root vertex $r \in \mathrm{V}$. There are $m$ monotone submodular functions $f_{1}, \ldots, f_{m}: 2^{V} \rightarrow \mathbb{R}_{+}$representing the valuations of different users. We assume without loss of generality by truncation that $f_{i}(V)=1$ for all $i \in[m]$. Function $f_{i}$ is said to be covered (or satisfied) by set $S \subseteq V$ if $f_{i}(S)=1=f_{i}(V)$. The cover time of function $f_{i}$ in a path $\pi$ is the length of the shortest prefix of $\pi$ that has $f_{i}$ value one, i.e.

$\min t: f_{i}(\{v \in V: v$ appears within distance $t$ on $\pi\})=1$.

The objective in the Minimum Latency Submodular Cover problem is to compute a path originating at $r$ that minimizes the sum of cover times of all functions.

We recover the Submodular Ranking problem [5] as the special case when metric $d$ is uniform. Similarly, we observe here that MLSC generalizes the Group Steiner Tree problem [43]. Note that

$$
f_{1}(S)=\frac{1}{N} \sum_{i=1}^{N} \min \left\{\left|g_{i} \cap S\right|, 1\right\}
$$

is a submodular function, with $f\left(S^{\prime}\right)=1$ if and only if $S^{\prime} \cap g_{i}$ is nonempty for all $i \in[N]$. So MLSC on metric $(V, d)$, root $r$ and one function $f_{1}$ is equivalent to the corresponding Group Steiner Tree problem (up to factor 2 due to converting a tree into a path). Hence Group Steiner Tree is a special case of MLSC with $m=1$ and $\epsilon=1 / N$. Similarly, Covering Steiner Tree, a generalization of Group Steiner Tree can be shown to be special case of MLSC.

CONTRIBUTIONS We introduce this problem and give an approximation algorithm that is very close to the best approximation algorithm known for Group Steiner Tree - a notoriously hard problem. The conceptual contribution is linking many problems, and providing a unified perspective on these problems which can enable further progress. 
Group Steiner Tree (CST) / Covering Steiner Tree (CST)

Given a metric $(V, d)$ with root $r$ and $N$ groups of vertices $\left\{g_{i} \subseteq V\right\}_{i=1}^{N}$ the goal is to find a minimum length tree containing $r$ and at least one vertex from each of the $\mathrm{N}$ groups.

The first poly-logarithmic approximation by Garg et al. for group Steiner tree was $\mathrm{O}\left(\log \mathrm{N} \log ^{2}|\mathrm{~V}|\right)$, which is still the best known bound[43]. Chekuri et al. [26] gave a combinatorial recursive greedy algorithm that achieved a slightly weaker approximation ratio (the algorithm in [43] is LP-based). This combinatorial approach was extended in Calinescu and Zelikovsky [21] to the problem of covering any submodular function in a metric space. We use this algorithm in the submodular orienteering (SOP) subroutine for our MLSC result. For SOP a $\mathrm{O}(\log |\mathrm{V}|)$-approximation is known due to Chekuri and Pal [28], but running in quasi-polynomial time. We note that an $\Omega\left(\log ^{2-\delta}|\mathrm{V}|\right)$ hardness of approximation is known for Group Steiner Tree (even on tree metrics) due to Halperin and Krauthgamer [58].

The Covering Steiner Tree problem is an extension of Group Steiner Tree. Given a metric $(V, d)$ with root $r$ and $N$ groups of vertices $\left\{g_{i} \subseteq V\right\}_{i=1}^{N}$ and requirements $\left\{k_{i}\right\}_{i=1}^{N}$, the goal is to find a minimum length tree containing $r$ and at least $k_{i}$ vertices from each group $g_{i}$.

The Covering Steiner Tree problem was introduced in Konjevod et al. [76], which can be viewed as the multicover version of Group Steiner Tree. They gave an $\mathrm{O}\left(\log \left(\mathrm{Nk}_{\max }\right) \log ^{2}|\mathrm{~V}|\right)$-approximation using an LP-relaxation. However the LP used in [76] has a large $\Omega\left(k_{\max }\right)$ integrality gap; they got around this issue by iteratively solving a suitable sequence of LPs. They also extended the randomized rounding analysis from [43] to this context. Later, Gupta and Srinivasan [54] improved the approximation bound to $\mathrm{O}\left(\log N \log ^{2}|\mathrm{~V}|\right)$, removing the dependence on the covering requirements. This algorithm was also based on solving a similar sequence of LPs; but the improvement was due to a combination of threshold-rounding and randomized rounding. In this paper, we give a stronger LP relaxation for Covering Steiner Tree based on so-called Knapsack-Covering-inequalities (abbreviated to KC-inequalities), that has an $\mathrm{O}\left(\log \mathrm{N} \log ^{2}|\mathrm{~V}|\right)$ integrality gap. This might be of independent interest. This new LP is also crucial for our $\mathrm{O}\left(\log ^{2}|\mathrm{~V}|\right)$-approximation algorithm for Latency Covering Steiner Tree.

CONTRIBUTIONS We develop a new linear programming formulation for Covering Steiner Tree with a polylogarithmic integrality gap. This new 
formulation allows to give an approximation algorithm with the same ratio as previously known, but only one linear program needs to be solved in contrast to a series of linear programs in the previous approach.

Latency Group Steiner Tree (LGST) / Latency Covering Steiner Tree (LCST)

Finally, we introduce the Latency Covering Steiner Tree problem. Given a metric $(V, d)$ with root $r$ and $N$ groups of vertices $\left\{g_{i} \subseteq V\right\}_{i=1}^{N}$, the goal is to find a path $\pi$ that minimizes the sum of of cover times of the groups. A group $g_{i}$ is covered at shortest prefix of $\pi$ that contains at least one vertex of $g_{i}$.

The latency group Steiner problem was studied in [53, 84] and also [23], in different contexts. These papers imply an $\mathrm{O}\left(\log ^{2}|\mathrm{~V}|\right)$-approximation algorithm for this problem.

The Latency Covering Steiner Tree problem is an extension of Latency Group Steiner Tree. Given a metric $(\mathrm{V}, \mathrm{d})$ with root $r$ and $\mathrm{N}$ groups of vertices $\left\{g_{i} \subseteq V\right\}_{i=1}^{N}$ and requirements $\left\{k_{i}\right\}_{i=1}^{N}$, find a path $\pi$ that minimizes the sum of of cover times of the groups. A group $g_{i}$ is covered at shortest prefix of $\pi$ that contains at least $k_{i}$ vertices of $g_{i}$.

CONTRIBUTIONS Using the linear program developed for Covering Steiner Tree we obtain a good approximation algorithm for Latency Covering Steiner Tree. 


\section{GENERALIZED MIN SUM SET COVER}

This chapter is based on the following paper:

S. Im, M. Sviridenko and R. van der Zwaan, "Preemptive and NonPreemptive Generalized Min Sum Set Cover" in International Symposium on Theoretical Aspects of Computer Science (STACS) 2012. Pages 465-476. [64].

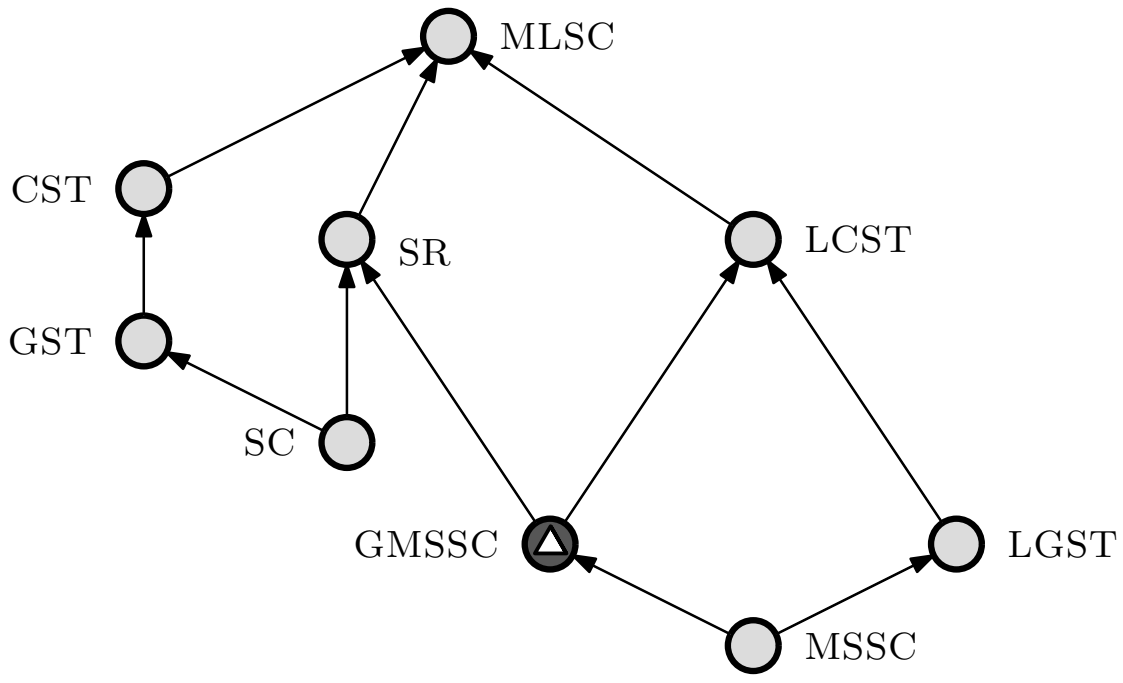




\subsection{INTRODUCTION}

In this chapter we study the Generalized Min Sum Set Cover problem. We improve previous results by looking at a relaxation, namely Preemptive Generalized Min Sum Set Cover.

We are given as input $n$ elements, $\{1,2, \ldots, n\}=[n]$ and a collection of sets $\mathcal{S}=\left\{S_{1}, S_{2}, \ldots, S_{m}\right\}$ where each set $S_{i} \in 2^{[n]}$ and for each set $S_{i}$ there is a requirement $k\left(S_{i}\right) \in\left[|| S_{i} \mid\right]$. The goal is to find a permutation of the elements such that the total sum of (or equivalently average) cover/hitting times of all sets is minimized. For simplicity, we will say that an element $e$ is covered at time slot $t$ or it has cover time $\operatorname{cov}(e)=t$ if it is placed in the $\mathrm{t}$-th position in the permutation. Equivalently, we may say that the element $e$ is scheduled at time $t$. The cover time $\operatorname{cov}\left(S_{i}\right)$ of a set $S_{i}$ with requirement $\kappa\left(S_{i}\right)$ is the earliest time $t$ such that $\left|\left\{e \in S_{i}: \operatorname{cov}(e) \leqslant t\right\}\right| \geqslant \kappa\left(S_{i}\right)$. The goal is to find a permutation of the elements in $[n]$ minimizing $\sum_{S_{i} \in \mathcal{S}} \operatorname{cov}\left(S_{i}\right)$.

Preemptive Generalized Min Sum Set Cover is defined as follows. Given the ground set of elements $[n]$, sets $\mathcal{S}=\left\{S_{1}, S_{2}, \ldots, S_{m}\right\}$ and requirement $\kappa(S) \in[|S|]$ for each set $S \in \mathcal{S}$, we should fractionally assign elements of the ground set to the interval $[0, n]$. Formally, we define functions $x_{e}(t):[0, n] \rightarrow\{0,1\}$ where $x_{e}(t)$ is the indicator function that denotes whether element $e$ is scheduled at time $t$ such that $\int_{t=0}^{n} x_{e}(t) d t=1$ for all $e \in[n]$ and $\sum_{e \in[n]} x_{e}(t)=1$ for any time $t \in[0, n]$. Then, the cover (or completion) time $\operatorname{cov}(S)$ of the set $S$ is defined as the earliest time $t$ such that $\int_{\tau=0}^{t} \sum_{e \in S} x_{e}(\tau) d \tau \geqslant k(S)$ and the goal is to minimize the sum of cover times over all sets. Note that the cover time $\operatorname{cov}(S)$ is not necessarily an integer unlike in the non-preemptive problem.

Our main motivation to study Preemptive Generalized Min Sum Set Cover is the fact that it provides a lower bound for the optimal value of the Generalized Min Sum Set Cover. We decouple finding an approximate solution to the relaxed problem (see Section 4.2) and the question of the lower bound quality (see Section 4.4 and Conjecture 4.1).

\subsubsection{Our Results}

Our main result is a polynomial time approximation algorithm with performance guarantee of 2 for the Preemptive Generalized Min Sum Set Cover. This result is tight modulo some complexity assumptions [9], see Section 1. We note that one can easily show that the linear program used in $[8,98]$ is a 
valid relaxation for the preemptive problem (see Proposition 4.19), thus the best known approximation for the non-preemptive problem also carries for the preemptive problem as well.

We introduce a configuration linear program which completely differs from the linear programming relaxation used in $[8,98]$. Interestingly, it is not obvious that our new linear program is a valid relaxation for the preemptive problem, unlike the previous linear program in $[8,98]$ which can be easily shown to be a valid relaxation for the preemptive (and non-preemptive) problem. Our new LP is provably stronger than the previous LP, for both the preemptive and non-preemptive problems.

Further, we study the "gap" between the preemptive and non-preemptive solutions of the Generalized Min Sum Set Cover Problem, which is of independent interest. With some modifications of the rounding scheme in [98], we show that one can transform any $\alpha$-approximate preemptive schedule into 6.2 $\alpha$-approximate non-preemptive one. With this transformation, we obtain a 12.4-approximation for the non-preemptive Generalized Min Sum Set Cover Problem, improving upon the previous best 28 -approximation by Skutella and Williamson [98]. We conjecture that the gap between optimal preemptive and non-preemptive solutions is precisely two.

All our proofs easily extend to the case where every set $S_{i}$ has a nonnegative weight $w_{i} \geqslant 0$ and the objective is to minimize $\sum S_{i} \in \mathcal{S} w_{i} \cdot \operatorname{cov}\left(S_{i}\right)$.

\subsubsection{Organization}

The remainder of this chapter is organized as follows. In Section 2 we introduce the configuration linear program $L P_{\text {primal }}$. First, in Section 4.2, we prove that our configuration linear program is a valid relaxation for Preemptive Generalized Min Sum Set Cover and that this linear program can be solved in polynomial time. Finally, we design a rounding procedure that results in a randomized 2-approximation (Section 4.2.4) that can be derandomized. In Section 4.3 we show that a 2-approximation is best possible, modulo some complexity assumptions. In Section 4.4 we obtain a transformation from a preemptive schedule to a non-preemptive schedule with a loss of factor 6.2, which immediately implies a 12.4-approximation in expectation to Generalized Min Sum Set Cover. In Section 4.5 we compare the time indexed linear programming relaxation used in $[8,98]$ to our configuration linear programming relaxation and show that our relaxation 
is stronger. We conclude this chapter with Section 5 with some open questions and conjectures.

\subsection{2-APPROXIMATION FOR PREEMPTIVE GENERALIZED MIN SUM SET COVER}

This section is devoted to proving the following theorem.

Theorem 4.1. There is a randomized polynomial time 2-approximation algorithm for Preemptive Generalized Min Sum Set Cover.

Throughout this section, for any integer $t \in[n]$, the $t$-th time slot will be equivalent to the time interval $(t-1, t]$.

\subsubsection{Configuration LP}

We write a configuration linear program. For a set $S \in \mathcal{S}$, a valid configuration is an (integral) assignment of elements in $S$ to time slots. More formally, such a map can be described as an injective function $f_{S}: S \rightarrow[n]$. For notational simplicity, we may represent the mapping via a relation (configuration) $F:=\left\{\left(e, f_{S}(e)\right) \mid e \in S\right\}$. Let $\mathcal{F}(S)$ denote the collection of all possible configurations for set $S$. Let $C_{S}^{F}$ denote the cover time $t$ of set $S$ under the configuration $F$, i.e. the first time $t^{\prime}$ such that $\left|f_{S}^{-1}\left(\left[t^{\prime}\right]\right)\right| \geqslant k(S)$. Let $x_{e, t}$ denote the fraction of element $e$ we schedule in the $t$-th time slot. The variable $y_{S}^{F}$ is used to indicate which configurations $S$ adheres to. For example, if $y_{S}^{F}=1$, it means all elements in $S$ are scheduled following the configuration $\mathrm{F}$.

Our integer program is formulated as follows. 


$$
\begin{array}{rr}
\min \sum_{S \in \mathcal{S}} \sum_{F \in \mathcal{F}(S)} C_{S}^{F} y_{S}^{F} & \\
\text { s.t. } \sum_{e} x_{e, t}=1 & \forall t \in[n] \\
\sum_{t}^{t} x_{e, t}=1 & \forall e \in[n] \\
\sum_{F \in \mathcal{F}(S)} y_{S}^{F}=1 & \forall S \in \mathcal{S} \\
\sum_{F \in \mathcal{F}(S),(e, t) \in F} y_{S}^{F}=x_{e, t} & \forall e, t \in[n], S: e \in S \\
x_{e, t} \in\{0,1\} & \forall e, t \in[n] \\
y_{S}^{F} \in\{0,1\} & \forall S \in \mathcal{S}, F \in \mathcal{F}(S)
\end{array}
$$

The constraints (I) and (2) enforce that exactly one element is scheduled at any time slot and that an element can be scheduled only once over all times. The constraint (3) states that each set $S$ has a unique configuration. Finally, (4) says that if an element $e$ is scheduled at time $t$, then it must align with the configuration of $S$.

The relaxation $L P$ primal of ILP is then defined as follows.

$$
\min \sum_{S \in \mathcal{S}} \sum_{F \in \mathcal{F}(S)} C_{S}^{F} y_{S}^{F} \quad\left(L P_{\text {primal }}\right)
$$

s.t. Constraints (1),(2),(3) and (4) hold

$$
\begin{array}{rr}
x_{e, t} \geqslant 0 & \forall e, t \in[n] \\
y_{S}^{F} \geqslant 0 & \forall S \in \mathcal{S}, F \in \mathcal{F}(S)
\end{array}
$$

\subsubsection{Validity of the LP}

It is easy to verify that $L P_{\text {primal }}$ is a valid linear programming relaxation for Generalized Min Sum Set Cover. However, it is not obvious that the $L P_{\text {primal }}$ is indeed a valid relaxation for the preemptive problem. Since we will use two different types of fractional schedules throughout the analysis, we first clearly define those schedules. The first one is a continuous schedule that is defined by indicator functions $x_{e}(t):[0, n] \rightarrow\{0,1\}, e \in[n]$ such that (1) for any $t \in[0, n], \sum_{e \in[n]} x_{e}(t)=1$ and (2) for any $e \in[n], \int_{\tau=0}^{n} x_{e}(\tau) d \tau=1$. 
We say that $x_{e}(t), e \in[n]$ is a feasible schedule if all these conditions are satisfied. Recall that the cover time $C_{S}$ of each set $S$ is defined by a continuous schedule as the earliest time $t$ such that $\int_{\tau=0}^{t} \sum_{e \in S} x_{e}(\tau) d \tau \geqslant \kappa(S)$. The other version of schedule, which is somewhat discretized, is defined by $x_{e, t}, e, t \in[n]$ that satisfy (1) $\sum_{e \in[n]} x_{e, t}=1$, (2) $\sum_{t \in[n]} x_{e, t}=1$ and (3) $0 \leqslant x_{e, t} \leqslant 1$ for any $e, t \in[n]$. When these conditions are satisfied, we will say $x_{e, t}, e, t \in[n]$ is feasible. Note that this discretized version of schedule does not immediately define the cover time of sets since it does not specify how the fractions of various elements are ordered within one time step. Rather, it is used in $L P_{\text {primal }}$ as a relaxation of continuous schedules. We show the following theorem.

Theorem 4.2. Consider any feasible continuous schedule $\left\{x_{e}(t)\right\}_{e \in[n]}$. Let $C_{S}$ denote the cover time of set $\mathrm{S}$ in this schedule. For any $\mathrm{e}, \mathrm{t} \in[\mathrm{n}]$, let $\mathrm{x}_{e, t}:=$ $\int_{\tau=t-1}^{t} x_{e}(\tau) d \tau$. Then $x_{e, t}$ satisfy constraints (1) and (2). Also there exists $y$ values that satisfy the other constraints (3) and (4) as well and further satisfy

$$
\sum_{S \in \mathcal{S}} \sum_{F \in \mathcal{F}(S)} C_{S}^{F} y_{S}^{F} \leqslant \sum_{S \in \mathcal{S}} C_{S}
$$

The first claim in Theorem 4.2 that $x_{e, t}$ satisfy constraints (I) and (2) easily follows from the properties of continuous schedules and from how $x_{e, t}$ are defined:

$$
\begin{aligned}
\sum_{e \in[n]} x_{e, t} & =\sum_{e \in[n]} \int_{\tau=t-1}^{t} x_{e}(\tau) d \tau \\
& =\int_{\tau=t-1}^{t} \sum_{e \in[n]} x_{e}(\tau) d \tau=\int_{\tau=t-1}^{t} 1 d \tau=1,
\end{aligned}
$$

and

$$
\sum_{t \in[n]} x_{e, t}=\sum_{t \in[n]} \int_{\tau=t-1}^{t} x_{e}(\tau) d \tau=\int_{\tau=0}^{n} x_{e}(\tau) d \tau=1 .
$$

In fact, it is not difficult to see that there exist $y$-values that satisfy all constraints (1)-(4). The following proposition however shows that not all such $y$-values serve our purpose. 
time

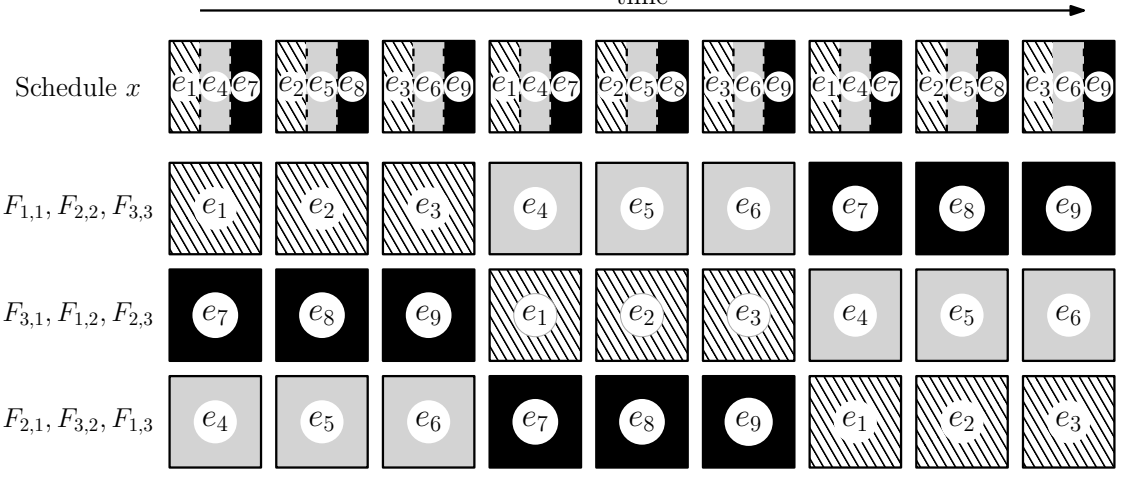

Figure 2: Elements from the sets $S_{1}, S_{2}, S_{3}$ are striped, gray and black. The top row depicts the fractional schedule, and the three lower rows depict the decomposition into configurations.

Proposition 4.3. There exist $\mathrm{y}_{\mathrm{S}}^{\mathrm{F}}$-values that satisfy (1)-(4), but not (5) within any constant factor.

Proof. Consider the following simple example. The inputs are $\mathcal{S}=\left\{\mathrm{S}_{1}=\right.$ $\left.\left\{e_{1}, e_{2}, e_{3}\right\}, S_{2}=\left\{e_{4}, e_{5}, e_{6}\right\}, S_{3}=\left\{e_{7}, e_{8}, e_{9}\right\}\right\}$ with $\kappa\left(S_{1}\right)=\kappa\left(S_{2}\right)=\kappa\left(S_{3}\right)=1$. The given schedule is as follows.

$$
\begin{aligned}
& -x_{e_{1} t}=x_{e_{2} t+1}=x_{e_{3} t+2}=1 / 3 \text { for all } t=1,4,7 . \\
& x_{e_{4} t}=x_{e_{5} t+1}=x_{e_{6} t+2}=1 / 3 \text { for all } t=1,4,7 . \\
& -x_{e_{7} t}=x_{e_{8} t+1}=x_{e_{9} t+2}=1 / 3 \text { for all } t=1,4,7 .
\end{aligned}
$$

Note that all sets $S_{1}, S_{2}$ and $S_{3}$ are completed at time 3 in the above schedule, i.e. $\mathrm{C}_{\mathrm{S}_{1}}=\mathrm{C}_{\mathrm{S}_{2}}=\mathrm{C}_{\mathrm{S}_{3}}=3$. Consider the following configurations and $y$-values that satisfy all constraints (I)-(4). See Figure 2.

- $F_{1,1}=\left\{\left(e_{1}, 1\right),\left(e_{2}, 2\right),\left(e_{3}, 3\right)\right\}, F_{1,2}=\left\{\left(e_{1}, 4\right),\left(e_{2}, 5\right),\left(e_{3}, 6\right)\right\}$,

$$
F_{1,3}=\left\{\left(e_{1}, 7\right),\left(e_{2}, 8\right),\left(e_{3}, 9\right)\right\} ; y_{S_{1}}^{F_{1,1}}=y_{S_{1}}^{F_{1,2}}=y_{S_{1}}^{F_{1,3}}=1 / 3 \text {. }
$$

- $F_{2,1}=\left\{\left(e_{4}, 1\right),\left(e_{5}, 2\right),\left(e_{6}, 3\right)\right\}, F_{2,2}=\left\{\left(e_{4}, 4\right),\left(e_{5}, 5\right),\left(e_{6}, 6\right)\right\}$,

$$
F_{2,3}=\left\{\left(e_{4}, 7\right),\left(e_{5}, 8\right),\left(e_{6}, 9\right)\right\} ; y_{S_{2}}^{F_{2,1}}=y_{S_{2}}^{F_{2,2}}=y_{S_{2}}^{F_{2,3}}=1 / 3 \text {. }
$$

- $F_{3,1}=\left\{\left(e_{7}, 1\right),\left(e_{8}, 2\right),\left(e_{9}, 3\right)\right\}, F_{3,2}=\left\{\left(e_{7}, 4\right),\left(e_{8}, 5\right),\left(e_{9}, 6\right)\right\}$,

$$
F_{3,3}=\left\{\left(e_{7}, 7\right),\left(e_{8}, 8\right),\left(e_{9}, 9\right)\right\} ; y_{S_{3}}=y_{S_{3}}=y_{S_{3}}=1 / 3 \text {. }
$$


The above configurations and $y$ variables give a left-hand-side for (5) of at least $1+4+7=12$. One can easily adapt this instance to make the left-hand-side arbitrarily greater than the right-hand-side.

Henceforth, we focus on showing that there exist "good" $y$-values that also satisfy (5). We will show how to construct a feasible solution y such that the inequality

$$
\sum_{F \in \mathcal{F}(S)} C_{S}^{F} y_{S}^{F} \leqslant C_{S}
$$

holds for any set $S \in \mathcal{S}$ which will imply the inequality (5). Since setting $y_{S}$-values for a specific $S$ does not affect other $y$-values, we can focus on each $S \in \mathcal{S}$ separately. We will find "good" $y_{S}^{F}$-values that satisfy constraints (3) and (4), and further (6). From this point on the analysis is focused on a fixed solution $x$ to the linear program and a single set $\mathrm{S}$.

To this end, we define two matroids $M_{1}$ and $M_{2}$ that enforce that any independent set in the intersection of $M_{1}$ and $M_{2}$ which in addition is a base in $M_{1}$ corresponds to a feasible configuration $F \in \mathcal{F}(S)$. Then we show that the vector $x_{e, t}, e \in S, t \in[n]$ lies in the intersection of the polytopes of the two matroids. Using the fact that such an intersection polytope is integral, we will be able to decompose $x$ into a convex combination of integer points that lie in the intersection of the polytopes of $M_{1}$ and $M_{2}$ (Theorem 2.6). As already mentioned, due to the structure of the matroids, each integer point will correspond to a configuration $F \in \mathcal{F}(S)$. By setting $y$ values as suggested by the decomposition, we will guarantee that $y$ satisfy constraints (3) and (4). Finally, we will complete the analysis by showing that such $y$-values satisfy (6) as well. This is enabled by some additional constraints we impose on the matroids. We refer the reader to Chapters 39-41 in [92] for an extensive overview of algorithmic matroid theory.

We begin with defining each of the two matroids $M_{1}$ and $M_{2}$ which have the same common ground set, $\mathrm{U}=\{(e, t) \mid e \in S, t \in[n]\}$ (Recall that we are focusing on each fixed $S \in \mathcal{S}$ separately). We will call $(e, t)$ a pair in order to distinguish it from elements, $[n]$. The first matroid $M_{1}=\left(U, \mathcal{J}\left(M_{1}\right)\right)$ enforces that each element in $\mathrm{S}$ can be scheduled in at most one time slot. Formally, the collection $\mathcal{J}\left(M_{1}\right)$ of independent sets of $M_{1}$ is defined as follows: $A \in \mathcal{J}\left(M_{1}\right)$ if and only if for any $e \in S,|A \cap\{(e, t) \mid t \in[n]\}| \leqslant 1$. Observe that $M_{1}$ is a partition matroid since pairs in $U$ are partitioned based on each common element, and any independent set collects at most 
one pair from each group. Hence the polytope $P\left(M_{1}\right)$ of $M_{1}$ is defined as follows.

$$
\begin{array}{rrr}
\sum_{\mathrm{t} \in[\mathrm{n}]} \mathrm{x}_{e, \mathrm{t}} \leqslant 1 \quad \quad \forall e \in S & \left(\mathrm{P}\left(\mathrm{M}_{1}\right)\right) \\
\mathrm{x}_{e, \mathrm{t}} \geqslant 0 & \forall \mathrm{e} \in \mathrm{S}, \mathrm{t} \in[\mathrm{n}]
\end{array}
$$

Proposition 4.4. The vector $\mathrm{x}=\left(\mathrm{x}_{\mathrm{e}, \mathrm{t}}\right), \mathrm{e} \in \mathrm{S}, \mathrm{t} \in[\mathrm{n}]$ is in the polytope $\mathrm{P}\left(\mathrm{M}_{1}\right)$. Moreover, $\sum_{e \in S, t \in[n]} x_{e, t}=|S|$.

Proof. For any $e \in S$, from the definition of $x_{e, t}$, we know that

$$
\sum_{t \in[n]} x_{e, t}=\sum_{t=1}^{n} \int_{\tau=t-1}^{t} x_{e}(\tau) d \tau=\int_{\tau=0}^{n} x_{e}(\tau) d \tau=1 .
$$

The second matroid $M_{2}=\left(U, J\left(M_{2}\right)\right)$ has a more involved structure. It enforces that in each time slot, at most one element in $S$ can be scheduled. Additionally, it enforces that at most $\kappa(S)$ elements can be scheduled during the first $\mathrm{C}-1$ time slots and at most $|S|-k(S)$ elements can be scheduled during the time slots, $C+1, C+2, \ldots, n$, where $C$ is an integer such that $C-1<C_{S} \leqslant C$. These additional constraints will be crucial in finding "good" $y$-values. Formally, $A \in \mathcal{J}\left(M_{2}\right)$ if and only if $A$ satisfies

- For each integer time $t \in[n],|A \cap\{(e, t) \mid e \in S\}| \leqslant 1$.

- $|A \cap\{(e, t) \mid e \in S, 1 \leqslant t \leqslant C-1\}| \leqslant k(S)$.

- $|A \cap\{(e, t) \mid e \in S, C+1 \leqslant t \leqslant n\}| \leqslant|S|-k(S)$.

We observe that $\mathcal{J}\left(M_{2}\right)$ is a laminar matroid: All pairs in $U$ are partitioned into groups with the same time $t$, and at most one pair can be chosen from each group to be in an independent set. Further, the second and third constraints put a limit on the number of pairs that can be chosen from the groups of time slots $t=1,2, \ldots, \mathrm{C}-1$ and from the groups of time slots 
$t=C+1, C+2, \ldots, n$, respectively. We define the polytope $P\left(M_{2}\right)$ of $M_{2}$ as follows.

$$
\begin{array}{rlr}
\sum_{e \in S} x_{e, t} & \leqslant 1 \quad \forall t \in[n] \\
\sum_{\mathrm{t}=1}^{C-1} \sum_{e \in S} x_{e, t} & \leqslant \kappa(S) \\
\sum_{\mathrm{t}=\mathrm{C}+1}^{\mathrm{n}} \sum_{e \in S} x_{e, \mathrm{t}} & \leqslant|S|-\kappa(S) \\
x_{e, t} & \geqslant 0 \quad \forall e \in S, t \in[n]
\end{array}
$$

Proposition 4.5. The vector $x=\left(x_{e, t}\right)$ lies in the polytope $\mathrm{P}\left(\mathrm{M}_{2}\right)$.

Proof. We begin with proving that $x_{e, t}$ satisfies the first constraint. From the definition of $x_{e, t}$, we have that

$$
\begin{aligned}
\sum_{e \in S} x_{e, t} & =\sum_{e \in S} \int_{\tau=t-1}^{t} x_{e}(\tau) d \tau \\
& =\int_{\tau=t-1}^{t} \sum_{e \in S} x_{e}(\tau) d \tau \leqslant \int_{\tau=t-1}^{t} 1 d \tau=1 .
\end{aligned}
$$

Now we consider the second constraint. Recall that $\mathrm{C}-1<\mathrm{C}_{\mathrm{S}} \leqslant \mathrm{C}$.

$$
\sum_{\mathrm{t}=1}^{\mathrm{C}-1} \sum_{e \in S} x_{e, t}=\int_{\tau=0}^{\mathrm{C}-1} \sum_{e \in S} x_{e}(\tau) d \tau \leqslant \int_{\tau=0}^{C_{S}} \sum_{e \in S} x_{e}(\tau) d \tau=\kappa(S)
$$

The last inequality is due to the definition of $C_{S}$. Finally,

$$
\begin{aligned}
\sum_{\mathrm{t}=\mathrm{C}+1}^{n} \sum_{e \in S} x_{e, t} & =\int_{\tau=C}^{n} \sum_{e \in S} x_{e}(\tau) d \tau \\
& \leqslant \int_{\tau=C_{S}}^{n} \sum_{e \in S} x_{e}(\tau) d \tau \\
& =|S|-\int_{\tau=0}^{C_{S}} \sum_{e \in S} x_{e}(\tau) d \tau \\
& =|S|-\kappa(S) .
\end{aligned}
$$


It is well known that the intersection of the independent set polytopes associated with two matroids is an integral polytope (See Theorem 2.6), i.e. any vertex point is integral. Hence since $\left(x_{e, t}\right)$ lies in the intersection of two polytopes $\mathrm{P}\left(\mathrm{M}_{1}\right)$ and $\mathrm{P}\left(\mathrm{M}_{2}\right)$, it can be decomposed into a linear combination of vertex (hence integer) points in $P\left(M_{1}\right) \cap P\left(M_{2}\right)$. Note that each of such integer points corresponds to an independent set in $\mathcal{J}\left(M_{1}\right) \cap$ $\mathcal{J}\left(M_{2}\right)$, which is of size at most $|S|$ due to the constraints of $M_{1}$. In fact, the size must be exactly $|S|$, since $\sum_{e \in S} \sum_{t \in[n]} x_{e, t}=|S|$. By the constraints of $M_{1}$ and the first constraints of $M_{2}$, we conclude that each of such integer points corresponds to a configuration $F \in \mathcal{F}(S)$. Hence we have shown the following lemma.

Lemma 4.6. There exist $\mathcal{F}^{\prime}(S) \subseteq \mathcal{F}(S)$ and positive constants $\theta_{S}^{\mathrm{F}}, \mathrm{F} \in \mathcal{F}^{\prime}(S)$ that satisfy

$$
\sum_{\mathrm{F} \in \mathcal{F}^{\prime}(S)} \theta_{S}^{\mathrm{F}}=1 ;
$$

- For any $e \in S, t \in[n], x_{e, t}=\sum_{F \in \mathcal{F}^{\prime}(S)} \theta_{S}^{F} \cdot \mathbf{1}[(e, t) \in F]$;

where an indicator variable $\mathbf{1}[(e, t) \in F]=1$ if and only if $(e, t) \in F$.

We let $y_{S}^{F}=\theta_{S}^{F}$ for all $F \in \mathcal{F}^{\prime}(S)$ and $y_{S}^{F}=0$ for all $F \in \mathcal{F}(S) \backslash \mathcal{F}^{\prime}(S)$. Note that $x$ and $y$ satisfy constraints (3) and (4).

It remains to show that $y$ satisfy (6). Now the second and third constraints of $M_{2}$ play a crucial role. We make the following observation.

Lemma 4.7. For any $\mathrm{F} \in \mathcal{F}^{\prime}(\mathrm{S})$ exactly one of the following holds.

- $|\mathrm{F} \cap\{(e, t) \mid e \in S, 1 \leqslant \mathrm{t} \leqslant \mathrm{C}-1\}|=\kappa(S)$.

- $|\mathrm{F} \cap\{(e, t) \mid e \in S, 1 \leqslant \mathrm{t} \leqslant \mathrm{C}-1\}|=\kappa(\mathrm{S})-1$ and $(e, C) \in \mathrm{F}$ for some $e \in S$.

Proof. Recall that $|\mathrm{F}|=|\mathrm{S}|$. By the third constraints of $\mathrm{M}_{2}$, we know that $N_{\geqslant C+1}:=|F \cap\{(e, t) \mid e \in S, C+1 \leqslant t \leqslant n\}| \leqslant|S|-k(S)$, hence that $N_{\geqslant c}:=|F \cap\{(e, t) \mid e \in S, C \leqslant t \leqslant n\}| \leqslant|S|-k(S)+1$. Therefore, we have $\mathrm{N}_{\leqslant C-1}:=|\mathrm{F} \cap\{(e, t) \mid e \in S, 1 \leqslant \mathrm{t} \leqslant \mathrm{C}-1\}| \geqslant k(S)-1$. Further, we know $N_{\leqslant C-1} \leqslant k(S)$ from the second constraint of $M_{2}$. Thus unless $N_{\leqslant C-1}=\kappa(S)$, it must be the case that $N_{\leqslant C-1}=\kappa(S)-1$. In that case, since $N_{\geqslant C+1} \leqslant|S|-\kappa(S)$, we conclude that $(e, C) \in F$ for some $e \in S$. 
Motivated by the above lemma, we can now prove that our linear program is a valid relaxation for the preemptive version of the problem.

Proof of Theorem 4.2. Partition $\mathcal{F}^{\prime}(S)$ into $\mathcal{F}_{1}^{\prime}(S)$ and $\mathcal{F}_{2}^{\prime}(S)$ by letting $\mathcal{F}_{1}^{\prime}(S)$ denote all $\mathrm{F} \in \mathcal{F}^{\prime}(S)$ that fall in the first case in Lemma 4.7 and letting $\mathcal{F}_{2}^{\prime}(S)=\mathcal{F}^{\prime}(S) \backslash \mathcal{F}_{1}^{\prime}(S)$. Let $\theta^{\prime}=\sum_{F \in \mathcal{F}_{2}^{\prime}(S)} \theta_{S}^{F}$. Note that for any $F \in \mathcal{F}_{1}^{\prime}(S)$, $\mathrm{C}_{\mathrm{S}}^{\mathrm{F}} \leqslant \mathrm{C}-1$ and for any $\mathrm{F} \in \mathcal{F}_{2}^{\prime}(\mathrm{S}), \mathrm{C}_{\mathrm{S}}^{\mathrm{F}}=\mathrm{C}$. In words, the set $\mathrm{S}$ is completed no later than time $C-1$ for $\left(1-\theta^{\prime}\right)$ fraction of configurations in $\mathcal{F}^{\prime}(S)$ and exactly at time $C$ for $\theta^{\prime}$ fraction of configurations in $\mathcal{F}^{\prime}(S)$. Hence we have that

$$
\begin{aligned}
\sum_{F \in \mathcal{F}(S)} C_{S}^{F} y_{S}^{F} & =\sum_{F \in \mathcal{F}^{\prime}(S)} C_{S}^{F} \theta_{S}^{F}=\sum_{F \in \mathcal{F}_{1}^{\prime}(S)} C_{S}^{F} \theta_{S}^{F}+\sum_{F \in \mathcal{F}_{2}^{\prime}(S)} C_{S}^{F} \theta_{S}^{F} \\
& \leqslant\left(1-\theta^{\prime}\right)(C-1)+\theta^{\prime} C=C-1+\theta^{\prime} .
\end{aligned}
$$

Now we focus on upper-bounding $\theta^{\prime}$. From the definition of $C_{S}$ and the fact that $\sum_{e \in S} x_{e}(\tau) \leqslant 1$ for any $\tau$, we know that

$$
\begin{aligned}
\int_{\tau=0}^{C-1} \sum_{e \in S} x_{e}(\tau) d \tau & =\int_{\tau=0}^{C_{S}} \sum_{e \in S} x_{e}(\tau) d \tau-\int_{\tau=C-1}^{C_{S}} \sum_{e \in S} x_{e}(\tau) d \tau \\
& \geqslant k(S)-\left(C_{S}-(C-1)\right) .
\end{aligned}
$$

On the other hand, it follows that

$$
\begin{aligned}
& \left.\int_{\tau=0}^{C-1} \sum_{e \in S} x_{e}(\tau) d \tau=\sum_{t=1}^{C-1} \sum_{e \in S} x_{e, t} \quad \text { [Definition of } x_{e, t}\right] \\
= & \sum_{t=1}^{C-1} \sum_{e \in S} \sum_{F \in \mathcal{F}^{\prime}(S):(e, t) \in F} y_{S}^{F} \quad\left[\text { Decomposition of } x \text { into } y_{S}^{F}\right] \\
= & \sum_{F \in \mathcal{F}_{1}^{\prime}(S)} y_{S}^{F} \sum_{e \in S} \sum_{t=1}^{C-1} \mathbf{T}[(e, t) \in F] \\
& +\sum_{F \in \mathcal{F}_{2}^{\prime}(S)} y_{S}^{F} \sum_{e \in S} \sum_{t=1}^{C-1} \mathbf{T}[(e, t) \in F] \\
= & \sum_{F \in \mathcal{F}_{1}^{\prime}(S)} \theta_{S}^{F} \cdot \kappa(S)+\sum_{F \in \mathcal{F}_{2}^{\prime}(S)} \theta_{S}^{F} \cdot(\kappa(S)-1) \\
= & \left(1-\theta^{\prime}\right) \cdot k(S)+\theta^{\prime} \cdot(\kappa(S)-1)=\kappa(S)-\theta^{\prime} .
\end{aligned}
$$


From 8) and (9), we have $\theta^{\prime} \leqslant C_{S}-(C-1)$. By combining this with (7), we complete the proof of Theorem 4.2:

$$
\begin{aligned}
\sum_{\mathrm{F} \in \mathcal{F}(\mathrm{S})} \mathrm{C}_{S}^{\mathrm{F}} \mathrm{y}_{S}^{\mathrm{F}} & \leqslant \mathrm{C}-1+\theta^{\prime} \\
& \leqslant \mathrm{C}-1+\mathrm{C}_{S}-(\mathrm{C}-1) \quad[(8) \text { and }(9)] \\
& \leqslant \mathrm{C}_{S}
\end{aligned}
$$

\subsubsection{Solving the LP}

The linear programming relaxation $\mathrm{LP}_{\text {primal }}$ has exponentially many variables. Hence, we solve the dual LP and show there are only polynomially many non-zero variables in the primal LP that achieve the optimal LP value. The dual LP is as follows.

$$
\begin{aligned}
& \max \sum_{\mathbf{t} \in[\mathrm{n}]} \alpha_{\mathrm{t}}+\sum_{\mathbf{e} \in[\mathrm{n}]} \beta_{e}+\sum_{\mathrm{S} \in \mathcal{S}} \gamma_{S}
\end{aligned}
$$

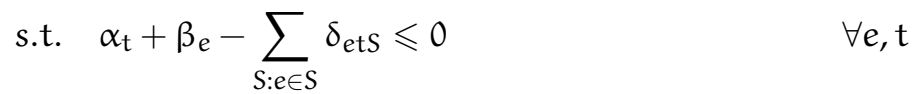

$$
\begin{aligned}
& \gamma_{S}+\sum_{(e, t) \in \mathrm{F}} \delta_{e t S} \leqslant \mathrm{C}_{S}^{\mathrm{F}} \quad \forall S \in \mathcal{S}, \mathrm{F} \in \mathcal{F}(\mathrm{S})
\end{aligned}
$$

To solve LP dual with the ellipsoid algorithm, we need a separation oracle for finding a violated constraint (Theorem 2.4 and see [51]). Since constraints (10) are easy to verify (there are only $n^{2}$ of them), we focus on constraints (II). We need a polynomial time algorithm that given $\gamma_{S}$ and $\delta_{\text {etS }}$-values, finds (if any) $S \in \mathcal{S}$ and $F \in \mathcal{F}(S)$ that violate constraints (II) or certifies that the solution is feasible.

We model this problem as a classical minimum cost $\mathrm{s}-\mathrm{t}$-flow problem. In this problem, we are given a digraph $G=(V, A)$, a capacity function $c: A \rightarrow \mathbb{Q}_{+}$, a cost function $k: A \rightarrow \mathbb{Q}$ and the volume $\phi \in \mathbb{Q}_{+}$. The goal is to send $\phi$ amount of flow from the source $s$ to the sink $t$, i.e. to find an $s-t$-flow $f$ of volume $\phi$, subject to capacity constraints $0 \leqslant f(e) \leqslant c(e)$ for all $e \in A$ and the standard flow conservation constraints, minimizing the costs $\sum_{e \in A} f(e) k(e)$.

It is known that if the volume $\phi$ and capacities $c_{e}, e \in E$ are integral then we can test in polynomial time if there is an $s-t$-flow of volume $\phi$. 


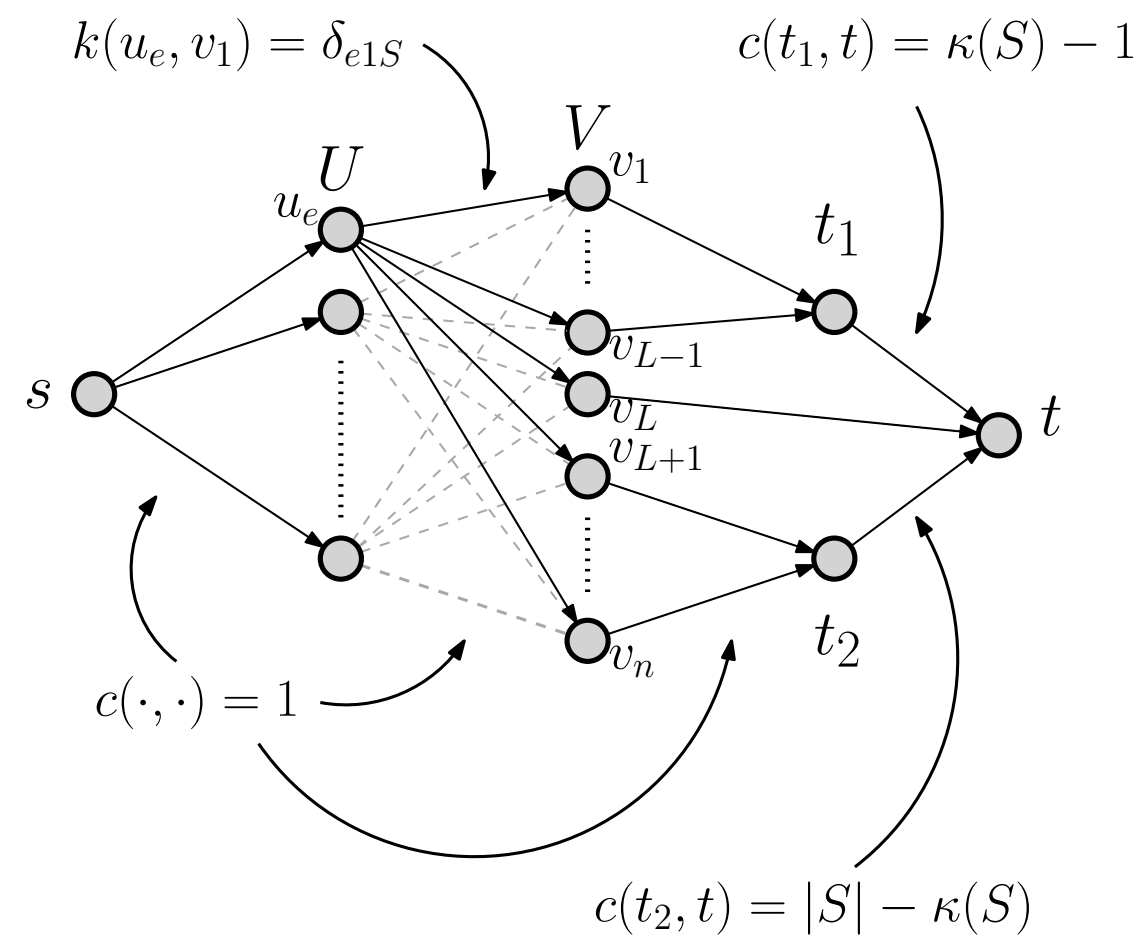

Figure 3: An illustration of the construction of the graph $G$, in which we want to find a maximum-value flow. 
Moreover, if there is such a flow (i.e. there is a feasible solution to the problem) then there is an integral minimum-cost $s-t$-flow, and it can be found in polynomial time (see Chapter 12 in [92]).

We now show how to reduce our separation problem for constraints (i1) to the minimum cost $s-t$-flow problem. It will be convenient for us to consider an equivalent maximum cost $s-t$-flow problem where the goal is to maximize the value of the objective function $\sum_{e \in A} f(e) k(e)$.

Fix a set $S$ and an integer $L \in[n]$. We will try to find a violated constraint for the constraints (II) corresponding to the set $S$ and configurations $F \in \mathcal{F}(S)$ with $C_{S}^{F}=L$. Create a directed complete bipartite graph $\mathrm{G}_{\mathrm{L}}=(\mathrm{U}, \mathrm{V}, \mathrm{A})$ where part $\mathrm{U}$ has vertex $\mathfrak{u}_{e}$ for each $e \in S$, part $\mathrm{V}$ has vertex $v_{i}$ for each time slot $i \in[n]$. Arc $a=\left(u_{e}, v_{i}\right) \in A$ has cost $k(e)=\delta_{\text {eis }}$ and capacity $c(e)=1$. We augment $G_{L}$ as follows. We add a source vertex $s$ and connect it to all vertices in $U$. There are two "intermediate" sinks $t_{1}$ and $t_{2}$, both connected to the "final" sink $t$. The vertices $v_{1}, v_{2}, \ldots, v_{\mathrm{L}-1}$ in $\mathrm{V}$ are connected to $t_{1}$ and the vertices $v_{\mathrm{L}+1}, v_{\mathrm{L}+2}, \ldots, v_{\mathrm{n}}$ in $\mathrm{V}$ are connected to the other intermediate sink $t_{2}$. The arcs a between the source $s$ and part $U$ have cost $k(a)=0$ and capacity $c(a)=1$. Analogously, all arcs a between part $V$ and intermediate sinks $t_{1}$ and $t_{2}$ have cost $k(a)=0$ and capacity $c(a)=1$. Arcs $a^{\prime}=\left(t_{1}, t\right)$ and $a^{\prime \prime}=\left(t_{2}, t\right)$ have capacities $c\left(a^{\prime}\right)=k(S)-1$ and $c\left(a^{\prime \prime}\right)=|S|-k(S)$ respectively, and all of them have zero costs. The vertex $v_{\mathrm{L}}$ is special and is directly connected to $t$. The $\operatorname{arc}\left(v_{\mathrm{L}}, \mathrm{t}\right)$ has a unit capacity and zero cost. The goal is to find the $s-t$-flow of volume $\phi=|S|$ of maximum cost. See Figure 3 for an illustration of this construction.

Note that any integral $s-t$-flow $f$ of value $|S|$ in digraph $G_{L}$ corresponds to a valid configuration $F$ for set $S$ such that $C_{S}^{F}=L$, and vice versa. Hence, if the maximum-cost $s-t$-flow in $G_{L}$ has cost more than $L-\gamma_{S}$, the constraint (II) is violated for $S$ and $F \in \mathcal{F}(S)$ that corresponds to the flow. The converse also holds: if the maximum-cost $s-t$-flow has cost less than or equal to $L-\gamma_{S}$ there is no configuration $F \in \mathcal{F}(S)$ with $C_{S}^{F}=L$ that violates (i1). With the help of this separation oracle and classical connection between separation and optimization [51], we can solve $L P_{\text {dual }}$ in polynomial time.

Then we can optimally solve $L P_{\text {primal }}$ by focusing only on $y_{S}^{F}$ variables that correspond to the constraints that were considered by the ellipsoid method in solving $L P_{\text {dual }}$. A more formal (and well-known) argument is that the $L P_{\text {dual }}$ with the subset of constraints considered by the ellipsoid method is a relaxation of the original problem but it has the same optimal solution. The dual of the relaxed problem is $L P_{\text {primal }}$ restricted to the subset 


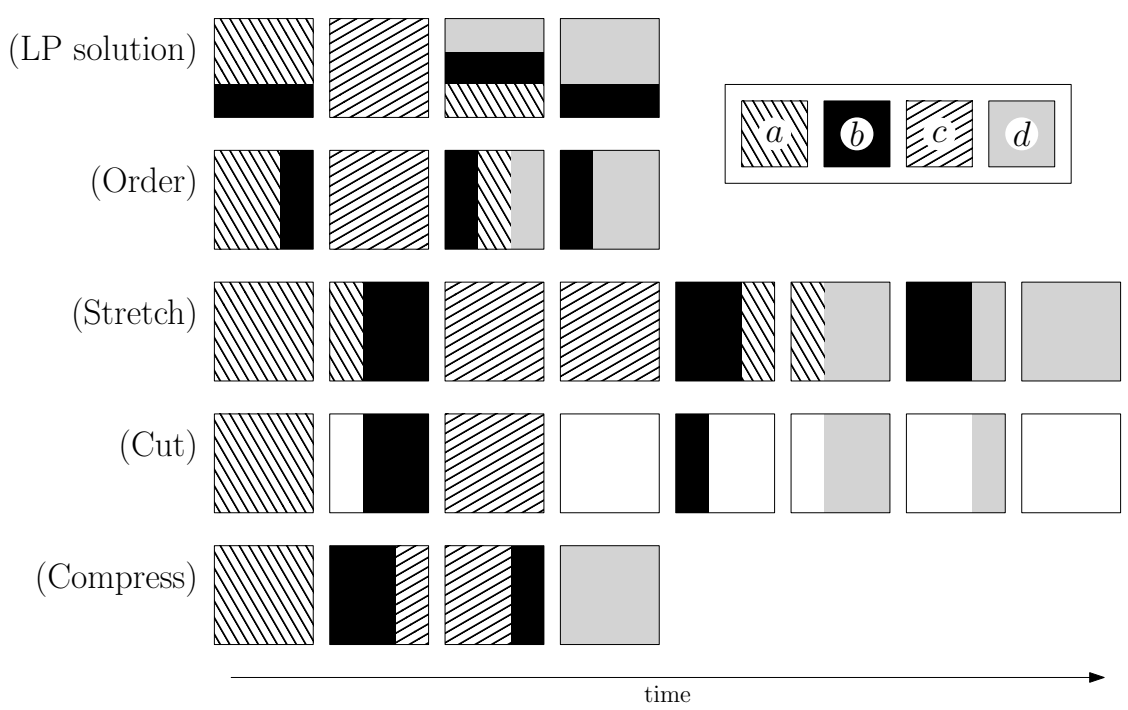

Figure 4: This figure illustrates the distinct steps for rounding a fractional schedule and depicts Example 4.1.

of corresponding variables which by the strong duality theorem has the same optimal value.

\subsubsection{Rounding procedure}

Let $x_{e, t}$ and $y_{S}^{F}$ be a extreme point optimal solution of the linear programming relaxation $L P_{\text {primal }}$. In particular we know that there are at most $2 n+m+n^{2} m$ non-zero variables (this is the number of constraints (1)-(4), Theorem 2.2). Let $C_{S}^{\mathrm{LP}}$ denote the cover time of set $S$ in the linear program solution. That is, $C_{S}^{L P}=\sum_{F \in \mathcal{F}(S)} C_{S}^{F} y_{S}^{F}$. We create a schedule parameterized by $\lambda \in(0,1]$, where $\lambda$ is randomly drawn from $(0,1]$ according to the density function $f(v)=2 v$.

Create an arbitrary continuous schedule $x_{e}(t), e \in[n], t \in[0, n]$ from $x_{e, t}, e, t \in[n]$ such that for any $e, t \in[n]$,

$$
\int_{\tau=t-1}^{t} x_{e}(\tau) d \tau=x_{e, t} .
$$

For example, this can be done by processing each element $e$ for the amount $x_{e, t}$ during the time step $t$ in an arbitrary order between the elements. For 
notational convenience, let $\sigma$ denote the continuous schedule $x_{e}(t)$. We will also use the standard machine scheduling terminology. The new schedule $\sigma(\lambda)$ is defined as follows. Stretch out the schedule $\sigma$ by a factor of $\frac{1}{\lambda}$. In other words, map every point $\tau$ in time onto $\tau / \lambda$. For each element $e$ define $\tau_{e} \in[1, n / \lambda]$ to be the earliest point in time when the element has been processed for one time unit (out of total $1 / \lambda$ ). Leave the machine idle whenever it processes the element $e$ after time $\tau_{e}$. After repeating this procedure for all elements $e \in[n]$, we shift the whole schedule to the left to eliminate all idle times. The final schedule $\sigma(\lambda)$ has total length $n$. Let $x_{e}^{(\lambda)}(t), e \in[n], t \in[0, n]$ be the resulting continuous schedule $\sigma(\lambda)$. Note that similar algorithms were used in scheduling before to design approximation algorithms for various preemptive scheduling problems with total cover time objective $[93,86]$.

Example 4.1. See Figure 4 for an illustration. Consider an instance with $4 \mathrm{el}-$ ements $\{a, b, c, d\}$, with the LP solution $x_{a, 1}=2 / 3, x_{b, 1}=1 / 3, x_{c, 2}=1$, $x_{\mathrm{d}, 3}=1 / 3, x_{\mathrm{b}, 3}=1 / 3, x_{\mathrm{a}, 3}=1 / 3, x_{\mathrm{d}, 4}=2 / 3, x_{\mathrm{b}, 4}=1 / 3$. Construct $a$ continuous schedule by randomly ordering the elements in each time step. For example in time step 3 , three elements, $\mathrm{a}, \mathrm{b}, \mathrm{d}$ are scheduled seamlessly, each for $1 / 3$ time steps. Then stretch the whole schedule by a factor two $(\lambda=1 / 2)$, and cut out each element after being scheduled by a unit amount. Finally, compress the schedule, by shifting everything to the left removing the idle times.

Let $C_{S}(\lambda)$ denote the cover time of $S$ in the new schedule $\sigma(\lambda)$. Order all configurations $F_{i} \in \mathcal{F}(S)$ for $y_{S}^{F}>0$ in non-decreasing order of $C_{S}^{F}$. Let $F_{1}, F_{2}, \ldots, F_{k}$ be such an ordering. Define $\tilde{C}_{S}(\lambda):=C_{S}^{F_{j}}$ where $\sum_{i=1}^{j-1} y_{S}^{F_{i}}<\lambda$ and $\sum_{i=1}^{j} y_{S}^{F_{i}} \geqslant \lambda$. See Figure 5 for an illustration.

Lemma 4.8. For any $S \in \mathcal{S}$ and $0<\lambda \leqslant 1, \mathrm{C}_{S}(\lambda) \leqslant \frac{1}{\lambda} \cdot \tilde{\mathrm{C}}_{S}(\lambda)$.

Proof. To simplify the proof we assume that there exists $j$ such that $\sum_{1 \leqslant l \leqslant j} y_{S}^{F_{l}}=$ $\lambda$. Otherwise, let $j$ be the lowest index such that $\sum_{1 \leqslant l \leqslant j} y_{S}^{F_{l}}>\lambda$, then we define two copies $F_{j}^{\prime}$ and $F_{j}^{\prime \prime}$ of configuration $F_{j}$, with $y_{S}^{F_{j}^{\prime}}=\lambda-\sum_{1 \leqslant l \leqslant j-1} y_{S}^{F_{l}}$ and $y_{S}^{F_{j}^{\prime \prime}}=\sum_{1 \leqslant l \leqslant j} y_{S}^{F_{l}} \lambda$. Here $F_{j}^{\prime}$ and $F_{j}^{\prime \prime}$ are the same configurations as $F_{j}$. Now, $\sum_{1 \leqslant l \leqslant j-1}\left(y_{S}^{F_{l}}\right)+y_{S}^{F_{j}^{\prime}}=\lambda$.

We will show the following inequality:

$$
\int_{\tau=0}^{\tilde{C}_{S}(\lambda) / \lambda} \sum_{e \in S} x_{e}^{(\lambda)}(\tau) d \tau \geqslant \kappa(S)
$$




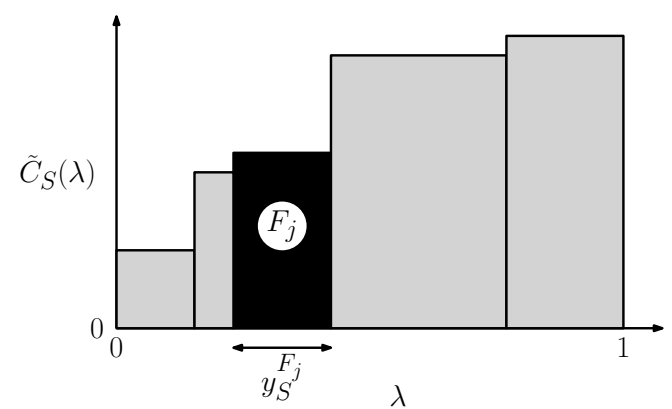

Figure 5: An illustration of the function $\tilde{C}_{S}(\lambda)$. The jth leftmost rectangle, which corresponds to $F_{j}$, has width $y_{S}^{F_{j}}$ and height $C_{S}^{F_{j}}$.

that would imply that the cover time $C_{S}(\lambda)$ of the set $S$ in the schedule $\sigma(\lambda)$ must be no later than $\tilde{C}_{S}(\lambda) / \lambda$. Since for every $e \in S$ we have

$$
\begin{aligned}
\int_{\tau=0}^{\tilde{C}_{s}(\lambda) / \lambda} x_{e}^{(\lambda)}(\tau) d \tau & \geqslant \min \left\{1, \frac{1}{\lambda} \int_{\tau=0}^{\tilde{C}_{s}(\lambda)} x_{e}(\tau) d \tau\right\} \\
& \geqslant \min \left\{1, \frac{1}{\lambda} \sum_{t \leqslant\left\lfloor\tilde{C}_{s}(\lambda)\right\rfloor} x_{e, t}\right\},
\end{aligned}
$$

and $\tilde{C}_{S}(\lambda)$ is integral by definition for any $\lambda \in(0,1]$, it is sufficient to show the inequality

$$
\sum_{e \in S} \min \left\{\lambda, \sum_{t \leqslant \tilde{C}_{S}(\lambda)} x_{e, t}\right\} \geqslant \lambda \kappa(S)
$$


to derive (12). We now derive the inequality (13).

$$
\begin{aligned}
& \sum_{e \in S} \min \left\{\lambda, \sum_{t \leqslant \tilde{C}_{S}(\lambda)} x_{e, t}\right\} \\
\geqslant & \sum_{e \in S} \min \left\{\lambda, \sum_{t \leqslant \tilde{C}_{S}(\lambda)} \sum_{l=1}^{j} y_{S}^{F_{l}} \cdot \mathbf{I}\left[(e, t) \in F_{l}\right]\right\} \\
= & \sum_{e \in S} \min \left\{\lambda, \sum_{l=1}^{j} y_{S}^{F_{l}} \cdot \mathbf{r}\left[(e, t) \in F_{l} \text { for some } t \leqslant \tilde{C}_{S}(\lambda)\right]\right\} \\
= & \sum_{e \in S} \sum_{l=1}^{j} y_{S}^{F_{l}} \cdot \mathbf{r}\left[(e, t) \in F_{l} \text { for some } t \leqslant \tilde{C}_{S}(\lambda)\right] \\
= & \sum_{l=1}^{j} y_{S}^{F_{l}} \sum_{e \in S} \mathbf{r}\left[(e, t) \in F_{l} \text { for some } t \leqslant \tilde{C}_{S}(\lambda)\right] \\
\geqslant & \sum_{l=1}^{j} y_{S}^{F_{l}} \kappa(S)=\lambda \kappa(S)
\end{aligned}
$$

The first inequality follows from constraints (4). The second equality holds because $\sum_{l=1}^{j} y_{S}^{F_{l}}=\lambda$. The last inequality holds because for any $F_{l}$, $l \leqslant j, C_{S}^{F_{l}} \leqslant \tilde{C}_{S}(\lambda)$.

The following lemma can be easily shown from the definition of $\tilde{C}_{S}(\lambda)$ (see also Figure 5).

Lemma 4.9. For any $S \in \mathcal{S}, \int_{\lambda=0}^{1} \tilde{C}_{S}(\lambda) d \lambda=C_{S}^{L P}$.

Proof. As before order all configurations $F_{i} \in \mathcal{F}(S)$ for $y_{S}^{F}>0$ in nondecreasing order of $C_{S}^{F}$. Let $F_{1}, F_{2}, \ldots, F_{k}$ be such an ordering.

Let $t_{0}, \ldots, t_{k}$ such that $t_{i}=\sum_{j=1}^{i} y_{S}^{F}$. Then

$$
\begin{aligned}
\int_{\lambda=0}^{1} \tilde{C}_{S}(\lambda) d \lambda & =\sum_{i=1}^{k} \int_{t_{i-1}}^{t_{i}} \tilde{C}_{S}(\lambda) d \lambda \\
& =\sum_{i=1}^{k} y_{S}^{F_{i}} \cdot C_{S}^{F_{i}} . \\
& =C_{S}^{L P} .
\end{aligned}
$$

The first equality is because we simply split the interval of integration into some multiple intervals and $t_{0}=0$ and $t_{k}=1$. The second equality is 
because $\tilde{C}_{S}(\lambda)$ is constant for $\lambda \in\left[t_{i-1}, t_{i}\right]$ and the final equality is by definition.

We now have all ingredients needed for the main theorem of this section. Proof of Theorem 4.I. By Theorem 4.2, $\mathrm{LP}_{\text {primal }}$ is a valid relaxation, and we now estimate the expected set cover time in the approximate solution.

$$
\begin{aligned}
\mathbb{E}\left[\mathrm{C}_{S}(\lambda)\right] & =\int_{\lambda=0}^{1} \mathrm{C}_{S}(\lambda) \cdot 2 \lambda d \lambda \quad[\text { By definition] } \\
& \leqslant \int_{\lambda=0}^{1} \frac{1}{\lambda} \cdot \tilde{\mathrm{C}}_{S}(\lambda) \cdot 2 \lambda \mathrm{d} \lambda \quad[\text { By Lemma } 4.8] \\
& =2 \int_{\lambda=0}^{1} \tilde{\mathrm{C}}_{S}(\lambda) \mathrm{d} \lambda \quad=2 \mathrm{C}_{S}^{\mathrm{LP}} \quad[\text { By Lemma 4.9] }
\end{aligned}
$$

We shortly indicate how our approximation algorithm can be derandomized. The function $\tilde{\mathrm{C}}_{S}(\lambda)$ is a piecewise constant function, with at most a polynomial number of pieces since there are at most polynomially many non-zero variables $y_{S}^{F}$ for each $S$. This implies that there are at most polynomially many "interesting" $\lambda$-values that we need to consider, among which at least one gives the desired approximation ratio.

\subsection{HARDNESS OF APPROXIMATION}

We show that for Preemptive Generalized Min Sum Set Cover there is no $2-\epsilon$ approximation for any $\epsilon>0$ assuming a variant of the Unique Games Conjecture.

The scheduling problem $1 \mid$ prec $\mid \sum w_{j} C_{j}$ is defined as follows. A set [ $\left.\mathrm{n}\right]$ of jobs has to be processed on a single machine, which can handle at most one job at a time. Each job $j$ has a positive processing time $p_{j}>0$ and a nonnegative weight $w_{j}>0$, and we want to find a schedule of the jobs that minimizes the weighted sum of job completion times, $\sum_{j \in[n]} w_{j} C_{j}$. Here, $\mathrm{C}_{j}$ denotes the time at which job $\mathrm{j}$ is completed in a feasible schedule. The precedence constraints are given in the form of a directed acyclic graph (i.e., a partial order) $G=([n], P)$, where $(i, j) \in P$ implies that job $i$ must be completed before job $j$ can be started. The precedence relation is transitive, so we assume that if $(i, j) \in P$ and $(j, k) \in P$ then $(i, k) \in P$. Further, the processing times are polynomial in $n$. 

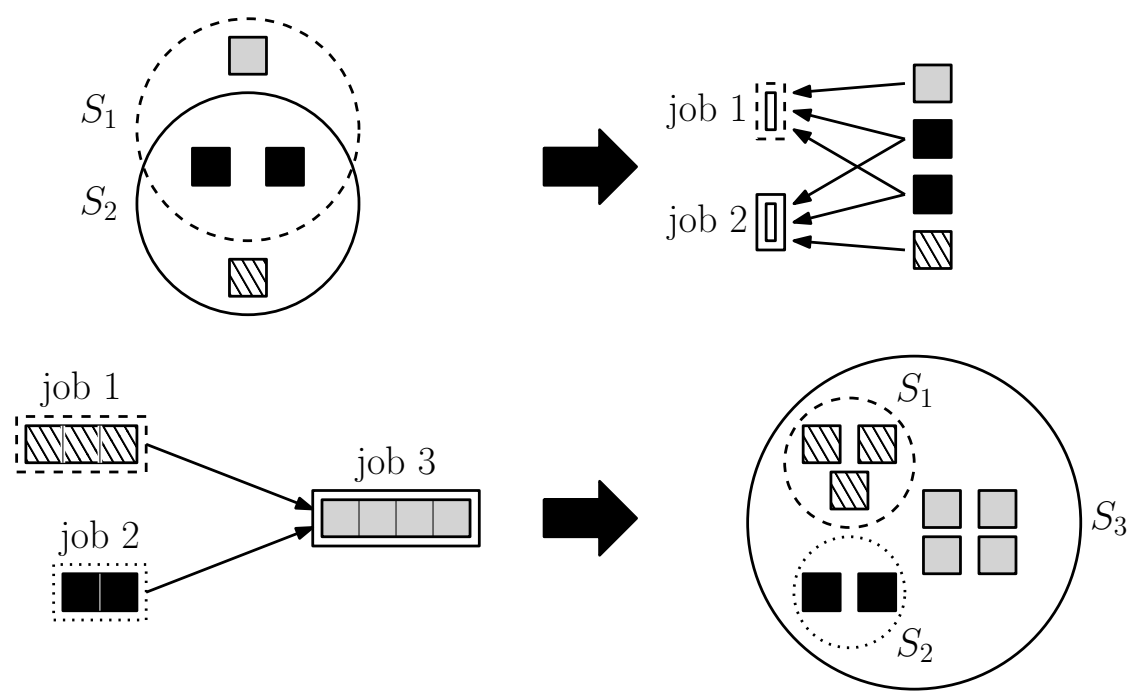

Figure 6: The reduction between Min Latency Set Cover and the single machine scheduling problem with precedence constraints. The figure on top depicts the transformation from a Min Latency Set Cover instance to the single machine scheduling problem, and the bottom figure depicts the reverse transformation.

Theorem 4.10 (Bansal and Khot, [9]). There is no polynomial time $2-\epsilon$ approximation algorithm for $1|\mathrm{prec}| \sum w_{j} \mathrm{C}_{\mathrm{j}}$ for any $\epsilon>0$ assuming the Unique Games Conjecture (Conjecture 2.4).

The Min Latency Set Cover problem is a variant where the cover time is defined as the time where all elements in the set are covered i.e. $\operatorname{cov}\left(S_{i}\right)=$ $\max _{e \in S_{i}} \operatorname{cov}(e)$.

Lemma 4.11. Min Latency Set Cover is equivalent to the single machine scheduling problem with precedence constraints $\left(1 \mid\right.$ prec $\left.\mid \sum w_{j} C_{j}\right)$.

Proof. The direction from Min Latency Set Cover to the machine scheduling problem $\left(1 \mid\right.$ prec $\left.\mid \sum w_{j} C_{j}\right)$ is simple. Given an instance with elements $[n]$ and sets $S_{1}, \ldots, S_{m}$ with weights $w_{1}, \ldots, w_{m}$. For each set $j$ create a job $a_{i}$ with weight $w_{j}$ and processing time 0 . For each element $i \in[n]$ create a job $b_{i}$ with processing time 1 and weight 0 . For every set $S_{j}$ and $i \in S_{j}$ we have that $b_{i}$ precedes $a_{j}$ i.e. $P=\left\{\left(b_{i}, a_{j}\right) \mid i \in S_{j}\right\}$. Correctness is easily verified.

The other direction is slightly more involved. Given jobs $1, \ldots, n$ with processing times $p_{1}, \ldots, p_{n}$ and weights $w_{1}, \ldots, w_{n}$ and precedence constraints 
$P=\{(i, j) \mid i$ precedes $j\}$. Without loss of generality we can assume that the processing times are integers. For each job $i$ create $p_{i}$ elements $P_{i}$ and for each job $i$ create a set $S_{i}$ with weight $w_{i}$ and with elements corresponding to all job that precede $i$ e.g. $S_{i}=P_{i} \cup \bigcup_{(j, i) \in P} P_{j}$.

Notice that the sets $S_{1}, \ldots, S_{n}$ form a laminar family, therefore any schedule can be transformed into a schedule that respects the precedence constraints of the original instance. Fix a set $S_{i}$ and ignore all time slots where no elements from $S_{i}$ are processed. Then, the elements $P_{i}$ that are specific to $S_{i}$ can be moved to the end of the schedule and the other elements can be pushed to earlier time slots and the cover times of the sets do not increase by this operation.

First we show with a simple exchange argument that preemption does not improve the solution quality.

Lemma 4.12. The optimal preemptive and non-preemptive schedule to Min Latency Set Cover have the same value.

Proof. Consider any optimal preemptive schedule and assume without loss of generality that the weights of all sets are 1 . Let $t_{1}$ and $t_{2}$ be two points in time such that: (a) at both $t_{1}$ and $t_{2}$ at least one set is covered and (b) no set is covered in the interval $\left(t_{1}, t_{2}\right)$.

Suppose that there is an element $e$ which is partially scheduled before $t_{1}$, then no set that is covered before or at $t_{1}$ contains $e$. Therefore, we can remove the parts of $e$ that are scheduled before $t_{1}$ and insert it at time $t_{1}$, shift the schedule to the left to remove the idle time. The schedule is still feasible and the value of the schedule decreased, therefore this contradicts with the premise that the schedule was optimal.

By the previous argument, for the interval $\left[t_{1}, t_{2}\right]$ we can assume that if part of an element $e$ is scheduled in $\left[t_{1}, t_{2}\right]$ then the whole element is scheduled in that interval. Clearly, we can reorder the elements in $\left[t_{1}, t_{2}\right]$ such that it is integral without increasing the cover times. Applying this argument for all intervals yields the lemma.

Hence we have the following theorem.

Theorem 4.13. For Preemptive Generalized Min Sum Set Cover there is no $2-\epsilon$ approximation for any $\epsilon>0$ assuming the Unique Games Conjecture (Conjecture 2.4). 


\subsection{GAP BETWEEN PREEMPTIVE AND NON-PREEMP- TIVE SCHEDULES}

In this section, we study the lower bound quality of the preemptive problem for the non-preemptive problem. Note that if we show a way to convert any given preemptive schedule into a non-preemptive one losing a factor of $\eta$, we would immediately obtain a $2 \eta$-approximation algorithm for the non-preemptive Generalized Min Sum Set Cover.

Our scheme for transforming a preemptive schedule into a non-preemptive one is similar to the one used by Skutella and Williamson [98]. We obtain a better gap by utilizing several additional tricks and starting from a preemptive schedule. Formally we will prove the following theorem.

Theorem 4.14. Given a preemptive schedule with cost $\mathrm{C}$, there exists a nonpreemptive schedule with expected cost at most 6.2C. Furthermore, this nonpreemptive schedule can be found in polynomial time.

Combining Theorem 4.1 and Theorem 4.14 we derive

Theorem 4.15. There exists an randomized polynomial time 12.4-approximation algorithm for Generalized Min Sum Set Cover.

Theorem 4.14 implies an upper bound on the gap of 6.2, and any gap lower than 2 would result in an approximation factor strictly less than 4 for the non-preemptive problem, which is impossible unless $\mathrm{P}=\mathrm{NP}$ [41].

The remaining section is devoted to proving Theorem 4.14. We start with defining several schedules that will be used throughout the analysis. Let $x_{e}(t), e \in[n], t \in[0, n]$ be a given preemptive solution where $x_{e}(t) \in\{0,1\}$ is the indicator function if an element $e$ is scheduled at time $t$. It would be convenient to extend the domain of $x_{e}(t)$ to $[0, \infty)$ by setting $x_{e}(t)=0$ for $t>n$. Let $C_{S}^{P}$ be the cover time of set $S$ in the preemptive schedule corresponding to the solution $x_{e}(t)$; the superscript $P$ stands for the preemptive schedule. Our goal is to randomly construct a non-preemptive schedule that completes set $S$ at time $C_{S}^{R}$ such that $\mathbb{E}\left[C_{S}^{R}\right]=O(1) C_{S}^{P}$. In the following we will use the notion of a solution (feasible or infeasible) interchangeably with the notion of a schedule.

We will define a new fractional solution (and a schedule) $\tilde{x}$ from $x$. Let $\sigma:=\sigma_{0}$ denote the schedule defined by $x$. For each integer $i \geqslant 1$, stretch out $\sigma$ horizontally by a factor of $r^{i}$ and let $\sigma_{i}$ be the resulting schedule. Here $r \geqslant 1$ is a constant to be fixed later. More formally, $x_{e}^{(i)}\left(r^{i} t\right)=\frac{1}{r^{i}} x_{e}(t)$ 
for all $e \in[n]$, defines the schedule $\sigma_{i}$. Note that now we allow $x_{e}^{(i)}(\tau)$ to be non-boolean: it denotes the rate at which we process element $e$. Note that $\sigma_{i}$ schedules element e during $\left[r^{i} t, r^{i}(t+d t)\right]$ by the same amount as $\sigma$ does during $[t, t+d t]$. For two parameters $Q \geqslant 0$ and $\rho \geqslant 0$, which we will fix later, define $\tilde{x}_{e}(t)$ as follows:

$$
\tilde{x}_{e}(t):=Q\left(x_{e}(t)+\rho \sum_{i=1}^{\infty} x_{e}^{(i)}(t)\right) .
$$

Note that $\tilde{x}_{e}(t)$ may not yield a feasible preemptive schedule because it may schedule elements at a rate of more than one at an instantaneous time. Let $\tilde{\sigma}$ denote the (infeasible) fractional schedule defined by $\tilde{x}_{e}(t)$. Via a randomized rounding, we will first obtain an intermediate infeasible integral schedule $\sigma^{\mathrm{I}}$ and then the final feasible integral schedule $\sigma^{\mathrm{R}}$. Throughout the analysis, we will be mostly concerned with the intermediate schedules $\tilde{\sigma}$ and $\sigma^{\mathrm{I}}$. In these schedules, we are allowed to schedule more than one elements at some times, and will define the cover time of sets in a natural way; the formal definitions will be given later when necessary.

Example 4.2. See Figure 7 for an illustration. We set $Q=2, \rho=1$ and $\mathrm{r}=2$. Consider an instance with 4 elements $\{\mathrm{a}, \mathrm{b}, \mathrm{c}, \mathrm{d}\}$, with a preemptive schedule $\left(x_{e}(t)\right)_{e \in[n]}:=\frac{3}{4} a, \frac{1}{4} b, 1 c, \frac{1}{2} b, \frac{1}{4} a, \frac{1}{4} d, \frac{1}{4} b, \frac{3}{4} d ;$ for example, $\frac{3}{4} a$ implies that element $\mathrm{a}$ is schedule for $\frac{3}{4}$ unit times. Then we create schedules $x^{(i)}$ from $x$ by stretching out $x$ horizontally by a factor $r^{i}$. By adding $Q$ copies of $x^{(0)}$ and $Q \rho$ copies of each $x^{(i)}, i \geqslant 1$, we obtain the "thick" schedule $\tilde{x}$.

Suppose $\alpha_{\mathrm{a}}=1 / 2, \alpha_{\mathrm{b}}=1 / 4, \alpha_{\mathrm{c}}=1$ and $\alpha_{\mathrm{d}}=1 / 2$. Then, $\mathrm{t}_{\mathrm{a}, \alpha_{\mathrm{a}}}=1 / 8$, $\mathrm{t}_{\mathrm{b}, \alpha_{\mathrm{b}}}=7 / 8, \mathrm{t}_{\mathrm{c}, \alpha_{\mathrm{c}}}=3 / 2$ and $\mathrm{t}_{\mathrm{d}, \alpha_{\mathrm{d}}}=3$.

The following lemma easily follows from the definition of $\tilde{x}_{e}(t)$.

Lemma 4.16. For any time $t \in[0, \infty)$,

$$
\int_{\tau=0}^{t} \sum_{e \in[n]} \tilde{x}_{e}(\tau) d \tau \leqslant Q\left(1+\frac{\rho}{r-1}\right) t .
$$

Proof. The desired bound easily follows by the definition of $\tilde{x}$ and the fact that $\sigma_{i}, i \geqslant 0$ schedules elements by an amount of at most $t / r^{i}$ until time t.

We now give our rounding procedure. For each $e \in[n]$, choose $\alpha_{e} \in[0,1]$ uniformly at random. Let $t_{e, \alpha_{e}}$ be the first time $t$ such that $\int_{0}^{t} \tilde{x}_{e}(\tau) d \tau \geqslant \alpha_{e}$. 


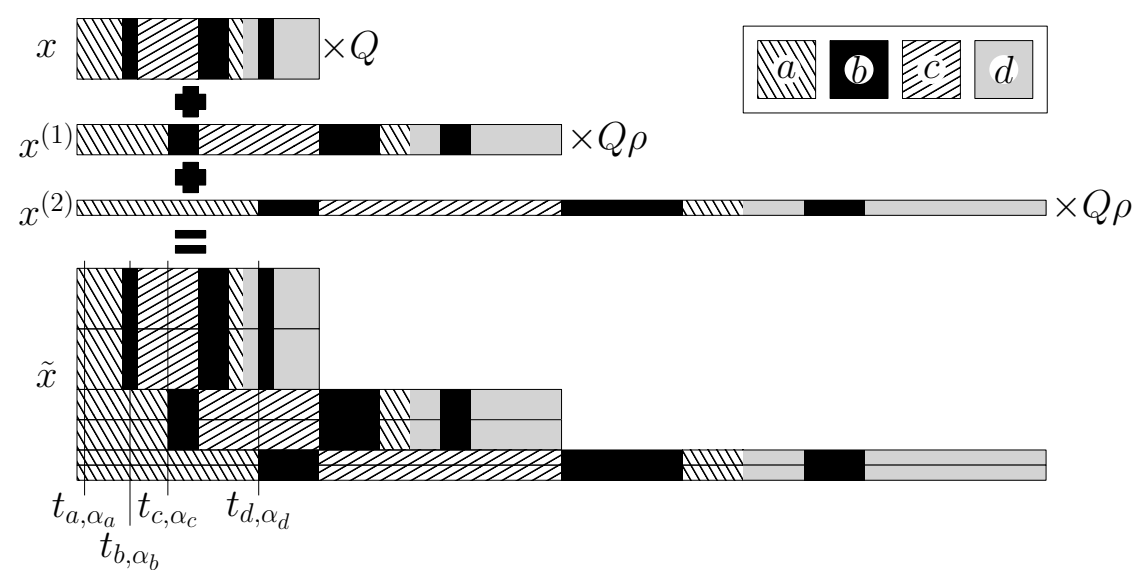

Figure 7: An illustration of the construction of schedule $\tilde{x}$ from $x$, with parameters $r=2, \rho=1$ and $Q=2$ and alpha values $\alpha_{a}=1 / 2, \alpha_{b}=1 / 4, \alpha_{c}=1$ and $\alpha_{\mathrm{d}}=1 / 2$.

Let $\sigma^{\mathrm{I}}$ denote the resulting (infeasible) schedule where $e$ is scheduled at time $t_{e, \alpha_{e}}$. Here when we say that element $e$ is scheduled at time $t_{e, \alpha_{e}}$ in $\sigma^{\mathrm{I}}$, we ignore that element $e$ takes a unit amount of time to be completely scheduled; this will be taken care of in the final schedule. Rather, we think of element $e$ as being tied to the instantaneous time $t_{e, \alpha_{e}}$. In the same spirit, we define the cover time $C_{S}^{\mathrm{I}}$ of $S$ in $\sigma^{\mathrm{I}}$ as the first time $t$ such that $\left|\left\{e \in S \mid t_{e, \alpha_{e}} \leqslant t\right\}\right| \geqslant k(S)$. We will schedule the elements in nondecreasing order of $t_{e, \alpha_{e}}$ as our final schedule $\sigma^{R}$, breaking ties arbitrarily. The algorithm is based on a popular scheduling concept of $\alpha$-points and similar to the one in [98].

Let $p_{S, i}$ denote the probability that $S$ is not satisfied until time $r^{i} \cdot C_{S}^{P}$ in $\sigma^{\mathrm{I}}$, i.e.

$$
p_{S, i}:=\operatorname{Pr}\left[\left|\left\{e \in S \mid t_{e, \alpha_{e}} \leqslant r^{i} \cdot C_{S}^{P}\right\}\right|<\kappa(S)\right]
$$

In the following lemma we upper bound $p_{i}:=\max _{S \in S} p_{S, i}$.

Lemma 4.17. Consider any $Q \geqslant 1, r>1$ and $\rho \leqslant 1$. Then we have $\max _{S} p_{S, i}=$ $p_{i} \leqslant \max \left\{K_{1 i}, K_{2 i}, K_{3 i}\right\}$ where

$$
\begin{aligned}
& \mathrm{K}_{1 i}=\exp (-\mathrm{Q}(1+\rho \mathrm{i})), \\
& \mathrm{K}_{2 \mathrm{i}}=\exp (-2 \mathrm{Q}(1+\rho \mathrm{i}))+2 \mathrm{Q}(1+\rho \mathrm{i}) \exp (-2 \mathrm{Q}(1+\rho \mathrm{i})+1), \\
& \mathrm{K}_{3 i}=\exp \left(-1.5 \mathrm{Q}\left(\left(1-\frac{1}{(1+\rho i) \mathrm{Q}}\right)^{2}(1+\rho i)\right)\right) .
\end{aligned}
$$


Proof. Consider any $\mathrm{S}$ and fixed $i \geqslant 0$. Let

$$
A:=\left\{e \in S: \int_{0}^{r^{i} \cdot C_{S}^{P}} \tilde{x}_{e}(\tau) d \tau \geqslant 1\right\} .
$$

Observe that any element $e \in A$ is scheduled no later than time $r^{i} \cdot C_{S}^{P}$ in the schedule $\sigma^{I}$. Note by definition of $C_{S}^{P}$ that $\sum_{e \in S} \int_{\tau=0}^{C_{S}^{P}} x_{e}(\tau) d \tau \geqslant k(S)$. Hence it follows (for all $A \subseteq S$ ) that

$$
\sum_{e \in S \backslash A} \int_{\tau=0}^{C_{S}^{P}} x_{e}(\tau) d \tau \geqslant \kappa(S)-|A| .
$$

Then from the definition of $\tilde{x}$ and by observing that for every $i \geqslant 0$ each $\sigma_{i}$ schedules elements in $S \backslash A$ by an amount of at least $\kappa(S)-|A|$ until time $r^{i} \cdot C_{S}^{P}$, for any $i \geqslant 0$,

$$
\sum_{e \in S \backslash A} \int_{\tau=0}^{r^{i} \cdot C_{S}^{P}} \tilde{x}_{e}(\tau) d \tau \geqslant Q(\kappa(S)-|A|)(1+\rho i) .
$$

For any $e \in S \backslash A$, let $X_{e}$ denote an indicator random variable such that $X_{e}=1$ if and only if $t_{e, \alpha_{e}} \leqslant r^{i} \cdot C_{S}^{P}$. Observe that $X_{e}$ are independent of each other, since the value of $X_{e}$ is determined by $\alpha_{e}$, which is randomly chosen independent of the other elements. Let $X:=\sum_{e \in S \backslash A} X_{e}$ and $\mu:=\mathbb{E}[X]$. Then, by observing that $\operatorname{Pr}\left[X_{e}=1\right]=\int_{\tau=0}^{r^{i} \cdot C_{S}^{P}} \tilde{X}_{e}(\tau) d \tau$, it follows that

$$
\mu=\mathbb{E}[X]=\sum_{e \in S \backslash A} \int_{\tau=0}^{r^{i} \cdot C_{S}^{P}} \tilde{x}_{e}(\tau) d \tau \geqslant Q \cdot(\kappa(S)-|A|) \cdot(1+\rho i) .
$$

Note that $p_{S, i} \leqslant \operatorname{Pr}[X<\kappa(S)-|A|]$. We consider a few cases depending on the value of $\kappa(S)-|A|$. Assume that $k(S)>|A|$, since otherwise $p_{S, i}=0$.

Case (a) : $\kappa(S)-|A|=1$. Using the fact that $X_{e}$ are independent of each other, we have

$$
\begin{aligned}
\operatorname{Pr}[X<\kappa(S)-|A|] & =\operatorname{Pr}[X=0] \\
& =\prod_{e \in S \backslash A}\left(1-\operatorname{Pr}\left[X_{e}=1\right]\right) \\
& \leqslant \exp \left(-\sum_{e \in S \backslash A} \operatorname{Pr}\left[X_{e}=1\right]\right)=\exp (-\mu) \\
& \leqslant \exp (-Q(1+\rho i)) .
\end{aligned}
$$


Case (b): $\kappa(S)-|A|=2$.

$$
\begin{aligned}
& \operatorname{Pr}[\mathrm{X}<\kappa(S)-|A|] \\
= & \operatorname{Pr}[\mathrm{X}=0]+\operatorname{Pr}[\mathrm{X}=1] \\
\leqslant & \exp (-\mu)+\sum_{e^{\prime} \in S \backslash A} \operatorname{Pr}\left[\mathrm{X}_{e^{\prime}}=1\right] \prod_{e \in S \backslash\left(A \backslash\left\{e^{\prime}\right\}\right)}\left(1-\operatorname{Pr}\left[X_{e}=1\right]\right) \\
\leqslant & \exp (-2 \mathrm{Q}(1+\rho i)) \\
& +\sum_{e^{\prime} \in S \backslash A} \operatorname{Pr}\left[X_{e^{\prime}}=1\right] \exp \left(-\sum_{e \in S \backslash\left(A \backslash\left\{e^{\prime}\right\}\right)} \operatorname{Pr}\left[X_{e}=1\right]\right) \\
\leqslant & \exp (-2 \mathrm{Q}(1+\rho i))+\mu \cdot \exp (-\mu+1) \\
\leqslant & \exp (-2 \mathrm{Q}(1+\rho i))+2 \mathrm{Q}(1+\rho i) \exp (-2 \mathrm{Q}(1+\rho i)+1)
\end{aligned}
$$

Case $(c): k(S)-|A| \geqslant 3$. This case can be done similarly as is done in [98]. The main difference is that here we are using the fact that $K-|A| \geqslant 3$, which helps to obtain a tighter bound.

$$
\begin{aligned}
\operatorname{Pr}[X<\kappa(S)-|A|] & \leqslant \operatorname{Pr}\left[X<\frac{\mu}{Q(1+\rho i)}\right] \\
& =\operatorname{Pr}\left[X<\mu\left(1-\left(1-\frac{1}{Q(1+\rho i)}\right)\right)\right] \\
& \leqslant \exp \left(-\frac{1}{2}\left(1-\frac{1}{Q(1+\rho i)}\right)^{2} \cdot \mu\right) \\
& \leqslant \exp \left(-\frac{1}{2}\left(1-\frac{1}{Q(1+\rho i)}\right)^{2} \cdot 3 Q(1+\rho i)\right)
\end{aligned}
$$

The second inequality comes from the Chernoff Bounds $\operatorname{Pr}[X<\mu(1-\delta)] \leqslant$ $\exp \left(-\frac{1}{2} \delta^{2} \mu\right)$ [82]. Taking the maximum of the bounds in the above three cases completes the proof.

In the following lemma, we bound the total expected cover time in the final schedule $\sigma^{R}$. Before giving a formal proof, we give a high-level explanation on how we obtain the upper bound in the lemma. First, the loss of factor $\mathrm{Q}\left(1+\frac{\rho}{\mathrm{r}-1}\right)$ comes from flatenning out the "thick" schedule $\tilde{\sigma}$ which was obtained by overlaying multiple schedules $\sigma_{i}$. The thickness is upperbounded in Lemma 4.16. The other term $\left(1+(r-1) \sum_{i=0}^{\infty} r^{i} p_{i}\right)$ follows from the definition of $p_{i}$ : Set $S$ is covered no later than time $r^{i} \cdot C_{S}^{P}$ with a probability of at least $1-p_{i}$. The final term comes from the following. 
In obtaining the final schedule $\sigma^{R}$, we count the expected number of elements that are scheduled before $C_{S}^{I}$ for each $S$. For a technical reason, those elements in $S$ are separately counted, which results in the final term.

Lemma 4.18. For any $\rho \leqslant 1, r>1$ and $Q \geqslant 1$, we have

$$
\mathbb{E}\left[\sum_{S \in \mathcal{S}} C_{S}^{R}\right] \leqslant Q\left(1+\frac{\rho}{r-1}\right)\left(1+(r-1) \sum_{i=0}^{\infty} r^{i} p_{i}\right) \sum_{S \in \mathcal{S}} C_{S}^{P}+\sum_{S \in \mathcal{S}} k(S) .
$$

Proof. Recall that $C_{S}^{I}$ is the earliest time $t$ such that $\mid\left\{e \in S: t_{e, \alpha_{e}} \leqslant\right.$ $t\} \mid \geqslant k(S)$. We first upper bound $\mathbb{E}\left[C_{S}^{I}\right]$. By definition of $p_{i}$, we have $\operatorname{Pr}\left[C_{S}^{I}>r^{i-1} \cdot C_{S}^{P}\right] \leqslant p_{i-1}$ for any $i \geqslant 1$. Thus it follows that

$$
\begin{aligned}
\mathbb{E}\left[C_{S}^{\mathrm{I}}\right] & \leqslant\left(\left(1-p_{0}\right)+\sum_{i=1}^{\infty} r^{i}\left(p_{i-1}-p_{i}\right)\right) C_{S}^{P} \\
& =\left(1+(r-1) \sum_{i=0}^{\infty} r^{i} p_{i}\right) C_{S}^{P} .
\end{aligned}
$$

As mentioned before, $C_{S}^{I}$ is not the cover time $C_{S}^{R}$ of $S$ in the final schedule $\sigma^{R}$. This is because we might have scheduled more than $C_{S}^{I}$ elements until time $C_{S}^{I}$. By counting the number of elements appearing no later than time $C_{S}^{I}$, we obtain the following relation between $C_{S}^{R}$ and $C_{S}^{I}$. Such elements are counted separately depending on whether they are in $S$ or not. We obtain

$$
C_{S}^{R} \leqslant\left|\left\{e \in[n] \backslash S \mid t_{e, \alpha_{e}} \leqslant C_{S}^{I}\right\}\right|+\kappa(S)
$$

Since for any fixed $e \notin S$ and time $t \in \mathbb{R}_{+}$,

$$
\operatorname{Pr}\left[t_{e, \alpha_{e}} \leqslant \mathrm{t}\right]=\min \left\{1, \int_{\tau=0}^{\mathrm{t}} \tilde{x}_{e}(\tau) \mathrm{d} \tau\right\}
$$

by Lemma 4.16, we have

$$
\begin{aligned}
\mathbb{E}\left[\left\{\left[e \in[n] \backslash S: t_{e, \alpha_{e}} \leqslant \mathrm{t}\right\}\right]\right] & \leqslant \sum_{e \in[n]} \int_{\tau=0}^{t} \tilde{x}_{e}(\tau) d \tau \\
& \leqslant Q\left(1+\frac{\rho}{r-1}\right) t .
\end{aligned}
$$


Therefore we have

$$
\begin{aligned}
& \left.\mathbb{E}\left[\left\{e \in[n] \backslash S \mid t_{e, \alpha_{e}} \leqslant C_{S}^{\mathrm{I}}\right\}\right]\right] \\
= & \int_{\tau=0}^{\infty}\left(\mathbb{E}\left[\left\{\left[e \in[n] \backslash S: t_{e, \alpha_{e}} \leqslant \tau\right\}\right] \cdot \operatorname{Pr}\left[C_{S}^{\mathrm{I}}=\tau\right]\right) d \tau\right. \\
\leqslant & \int_{\tau=0}^{\infty}\left(\mathrm{Q}\left(1+\frac{\rho}{r-1}\right) \tau \cdot \operatorname{Pr}\left[C_{S}^{\mathrm{I}}=\tau\right]\right) d \tau \\
\leqslant & Q\left(1+\frac{\rho}{r-1}\right) \int_{\tau=0}^{\infty}\left(\tau \cdot \operatorname{Pr}\left[C_{S}^{\mathrm{I}}=\tau\right]\right) d \tau \\
= & Q\left(1+\frac{\rho}{r-1}\right) \mathbb{E}\left[C_{S}^{\mathrm{I}}\right] .
\end{aligned}
$$

Hence it follows that

$$
\begin{aligned}
\mathbb{E}\left[C_{S}^{R}\right] & \leqslant Q\left(1+\frac{\rho}{r-1}\right) \mathbb{E}\left[C_{S}^{I}\right]+\kappa(S) \\
& \leqslant Q\left(1+\frac{\rho}{r-1}\right)\left(1+(r-1) \sum_{i=1}^{\infty} r^{i} p_{i}\right) C_{S}^{P}+\kappa(S) .
\end{aligned}
$$

Summing over all sets $S$ completes the proof.

We are now ready to complete the proof of Theorem 4.I4.

Proof of Theorem 4.14. Observe that $\sum_{S \in \mathcal{S}} K(S)$ is a lower bound on the cost of any preemptive schedule. From Lemma 4.18, it suffices to show

$$
\mathrm{Q}\left(1+\frac{\rho}{\mathrm{r}-1}\right)\left(1+(\mathrm{r}-1) \sum_{i=0}^{\infty} \mathrm{r}^{i} \mathrm{p}_{\mathrm{i}}\right) \leqslant 5.2
$$

We set $\mathrm{Q}=2.65, \mathrm{r}=1.40$ and $\rho=0.22$. By applying the upper bound in Lemma 4.17, we obtain $Q\left(1+\frac{\rho}{r-1}\right)\left(1+(r-1) \sum_{i=0}^{30} r^{i} p_{i}\right) \leqslant 5.13$ (the computation was done numerically). For $i>30$, we now prove that

$$
\begin{aligned}
r^{i} p_{i} & \leqslant r^{i} \max \left(K_{1 i}, K_{2 i}, K_{3 i}\right) \\
& \leqslant 2 \exp (-Q(1+\rho i)) r^{i} \\
& <1.145 \cdot\left(e^{-Q \rho} r\right)^{i} \\
& <1.145 \cdot(0.782)^{i} .
\end{aligned}
$$

From Lemma 4.17, we derive

1. $\mathrm{K}_{1 i}=\leqslant 2 \exp (-\mathrm{Q}(1+\rho i))$; 
2. Using the fact that $2 e \frac{z}{\exp (z)} \leqslant 1$ for $z \geqslant 3$ and $Q(1+\rho i) \geqslant 2.65(1+30$. $0.22)>10$, it follows that

$$
\begin{aligned}
\mathrm{K}_{2 i} & =\exp (-2 \mathrm{Q}(1+\rho i))+2 \mathrm{Q}(1+\rho i) \exp (-2 \mathrm{Q}(1+\rho i)+1) \\
& =\exp (-2 \mathrm{Q}(1+\rho i))+2 e \frac{\mathrm{Q}(1+\rho i)}{\exp (\mathrm{Q}(1+\rho i))} \exp (-\mathrm{Q}(1+\rho i)) \\
& \leqslant 2 \exp (-\mathrm{Q}(1+\rho i)) .
\end{aligned}
$$

3. Finally, from the fact that $Q(1+\rho i)>10$, we know

$$
\begin{aligned}
\mathrm{K}_{3 i} & =\exp \left(-1.5 \mathrm{Q}\left(\left(1-\frac{1}{(1+\rho i) \mathrm{Q}}\right)^{2}(1+\rho i)\right)\right) \\
& \leqslant \exp \left(-1.5 \mathrm{Q}(1+\rho \mathrm{i})(1-1 / 10)^{2}\right) \\
& \leqslant \exp (-1.2 \mathrm{Q}(1+\rho \mathrm{i})) \\
& \leqslant 2 \exp (-\mathrm{Q}(1+\rho i)) .
\end{aligned}
$$

Hence it follows that

$$
\begin{aligned}
& \mathrm{Q}\left(1+\frac{\rho}{r-1}\right)\left((r-1) \sum_{i=31}^{\infty} r^{i} p_{i}\right) \\
< & \mathrm{Q}\left(1+\frac{\rho}{r-1}\right)\left(1.145(r-1) \sum_{i=31}^{\infty}(0.782)^{i}\right) \\
= & \mathrm{Q}\left(1+\frac{\rho}{r-1}\right)\left(1.145(r-1)(0.782)^{31} \sum_{i=0}^{\infty}(0.782)^{i}\right) \\
< & \mathrm{Q}\left(1+\frac{\rho}{r-1}\right)\left(1.145(r-1)(0.782)^{31} \cdot \frac{1}{1-0.782}\right)<0.02
\end{aligned}
$$

We also need to add one due to the last term in the Lemma 4.18. This establishes that the gap between between preemptive and non-preemptive schedules for the min sum objective is at most 6.2.

\subsection{COMPARISON OF LINEAR PROGRAMMING FOR- MULATIONS}

In this section, we demonstrate that our configuration LP (LP Primal $)$ is stronger for both the non-preemptive and preemptive problems than the LP (LPBGK) 
considered in $[8,98]$, which is based on the knapsack cover inequalities. For completeness, we present $L P_{B G K}$ as follows.

$$
\begin{aligned}
& \min \sum_{t \in[n]} \sum_{S \in \mathcal{S}}\left(1-z_{S, t}\right) \\
& \text { s.t. } \quad \sum_{e \in[n]} x_{e, t}=1 \\
& \sum_{t \in[n]} x_{e, t}=1 \\
& \sum_{e \in S \backslash A} \sum_{t^{\prime}<t} x_{e, t^{\prime}} \quad \geqslant(\kappa(S)-|A|) \cdot z_{S, t} \\
& \forall \mathrm{S} \in \mathcal{S}, \forall \mathrm{A} \subseteq \mathrm{S}, \forall \mathrm{t} \in[\mathrm{n}] \\
& x_{e, t} \geqslant 0 \\
& \forall e \in[\mathrm{n}], \forall \mathrm{t} \in[\mathrm{n}] \\
& z_{S, t} \in[0,1] \\
& \forall \mathrm{t} \in[\mathrm{n}] \\
& \forall e \in[\mathrm{n}] \\
& \forall \mathrm{S} \in \mathcal{S}, \forall \mathrm{t} \in[\mathrm{n}]
\end{aligned}
$$

Note that the constraints (I) and (2) in LP Primal are exactly as the same as (15) and (16) in $L P_{B G K}$. In $L P_{B G K}$, the objective is based on the variables $z_{S, t}$, the extent to which $S$ is covered until time $t-1$. Each set $S$ contributes to the objective by $\left(1-z_{S, t}\right)$ at each time $t$. The variables $z_{S, t}$ are defined by the knapsack cover inequalities. We show that $L P_{B G K}$ is also a valid relaxation for the preemptive problem as well as for the non-preemptive one, as is LPPrimal.

Proposition 4.19. $\mathrm{LP}_{\mathrm{BGK}}$ is a valid linear programming relaxation for Preemptive Generalized Min Sum Set Cover.

Proof. Consider any preemptive schedule $x_{e}(t), e \in[n], t \in[0, n]$. Define the value of $x_{e, t}:=\int_{\tau=t-1}^{t} x_{e}(\tau) d \tau$ from $x_{e}(t)$. Recall in Theorem 4.2 that we have shown that $x_{e, t}$ satisfy constraints (15) and (16). For each set $S \in \mathcal{S}$ and $\mathrm{t} \in[\mathrm{n}]$, let $z_{S, t}=\min _{\mathrm{A} \subseteq S,|A|<\mathrm{K}(S)} \frac{\sum_{e \in S \backslash A} \sum_{\mathrm{t}^{\prime}<\mathrm{t}} \mathrm{x}_{e, \mathrm{t}^{\prime}}}{\mathrm{k}(S)-|A|}$. Then, constraints (17) are clearly satisfied.

We now shift our attention to proving that for the $x_{e, t}, z_{S, t}$ values we defined above, $L P_{B G K}$ has an objective smaller than the total preemptive cover time under the continuous schedule $x_{e}(t)$. To this end, it suffices to show that for each set $S \in \mathcal{S}, \sum_{t \in[n]}\left(1-z_{S, t}\right) \leqslant C_{S}^{P}$, where $C_{S}^{P}$ be the (preemptive) cover time of $S$. Consider any set $S \in \mathcal{S}$. First observe that for any time $t \geqslant\left\lfloor C_{S}^{P}\right\rfloor+2, z_{S, t}=0$, since at least $k(S)$ elements are completely 
scheduled from $S$ by time $\left\lfloor C_{S}^{P}\right\rfloor+1$. Now we consider the time $\left\lfloor C_{S}^{P}\right\rfloor+1$. Let $S^{\prime} \subseteq S$ be a subset of $k(S)$ elements that are completely scheduled by time $C_{S}^{P}$. Hence it must be the case that for each element $e \in S^{\prime}, \int_{\tau=0}^{\left\lfloor C_{S}^{P}\right\rfloor} x_{e}(\tau) d \tau \geqslant$ $1-\left(C_{S}^{P}-\left\lfloor C_{S}^{P}\right\rfloor\right)$. As such, $z_{S,\left\lfloor C_{S}^{P}\right\rfloor} \geqslant 1-\left(C_{S}^{P}-\left\lfloor C_{S}^{P}\right\rfloor\right)$. Finally, for each time $t \leqslant\left\lfloor C_{S}^{P}\right\rfloor, S$ adds at most 1 to the $L P_{\text {Primal }}$ objective. In sum, we obtain $\sum_{t \in[n]}\left(1-z_{S, t}\right) \leqslant\left\lfloor C_{S}^{P}\right\rfloor+\left(1-z_{S,\left\lfloor C_{S}^{P}\right\rfloor}\right) \leqslant C_{S}^{P}$.

We now focus on showing that our relaxation $L P_{\text {Primal }}$ gives a stronger lower bound than $L P_{B G K}$. We first provide an instance for which $L P_{\text {Primal }}$ has an objective value strictly larger than $L P_{B G K}$.

Proposition 4.20. For any $\epsilon>0$, there exists an instance for which $\mathrm{LP}_{\text {Primal }}$ has an objective larger than the objective of $\mathrm{LP}_{\mathrm{BGK}}$ by a factor of more than $2-\epsilon$.

Proof. Consider the instance where there exists a single set $S=[n]$ with $\kappa(S)=n$. We first consider the objective of $L P_{\text {Primal }}$. Since all configurations $F \in \mathcal{F}(S)$ have $C_{S}^{F} \geqslant n, L P_{\text {Primal }}$ gives a solution with cost no smaller than $n$. Now we turn our attention to $L P_{B G K}$. Consider the schedule where all elements are equally scheduled in each time slot, i.e. $x_{e, t}=1 / n$ for all $e, t \in[n]$. Since $z_{S, t}=\frac{t-1}{n}$ satisfies the constraints, $L P_{B G K}$ will have an objective value of $\sum_{t=1}^{n}\left(1-\frac{t-1}{n}\right)=\frac{n+1}{2}$. The claim immediately follows.

We now show the following lemma. Since both linear programs, $L P_{\text {Primal }}$ and $L P_{B G K}$ are valid relaxations, it, together with the above proposition, will establish that $L P_{\text {Primal }}$ is stronger than $L P_{B G K}$.

Lemma 4.21. For any instance, $\mathrm{LP}_{\text {Primal }}$ has an objective no smaller than the objective of $L P_{\mathrm{BGK}}$.

Proof. Consider an arbitrary instance and let $x^{*}, y^{*}$ be a fixed optimal solution to $L P_{\text {Primal }}$. Define $z$ as follows:

$$
z_{S, t}:=\sum_{F \in \mathcal{F}(S) \text { and } C_{S}^{F}<t} y_{S}^{* F}
$$


We first show that $x^{*}, z$ satisfy all constraints in $L P_{B G K}$. We focus on showing constraints (17), since all the other constraints are trivially satisfied. Consider any $S$ and $A \subseteq S$. Then we have

$$
\begin{aligned}
& \sum_{e \in S \backslash A} \sum_{t^{\prime}<t} x_{e, t^{\prime}}^{*} \\
= & \sum_{e \in S \backslash A} \sum_{t^{\prime}<t} \sum_{F \in \mathcal{F}(S),\left(e, t^{\prime}\right) \in F} y_{S}^{* F} \quad[\text { From constraints (4)] } \\
= & \sum_{F \in \mathcal{F}(F)} y_{S}^{* F} \sum_{e \in S \backslash A} \sum_{t^{\prime}<t} \mathbf{I}\left[\left(e, t^{\prime}\right) \in F\right] \\
\geqslant & \sum_{F \in \mathcal{F}(F) \text { and }} C_{S}^{F}<t \\
\geqslant & \sum_{S}^{* F} \sum_{e \in S \backslash A} \sum_{t^{\prime}<t} \mathbf{I}\left[\left(e, t^{\prime}\right) \in F\right] \\
= & \left(K \in \mathcal{F}(F) \text { and } C_{S}^{F}<t\right. \\
& \left.y_{S}^{* F}(K)-|A|\right) \cdot z_{S, t}
\end{aligned}
$$

The last inequality holds because for any $t \in[n], S \in \mathcal{S}, F \in \mathcal{F}(S)$ with $C_{S}^{F}<t$, at least $k(S)$ elements in $S$ are scheduled before time $t$ under the configuration $\mathrm{F}$.

Let $\operatorname{LP}_{\text {Primal }}\left(x^{*}, y^{*}\right)$ denote the objective of $L P_{\text {Primal }}$ by the solution $x^{*}, y^{*}$. Likewise $\operatorname{LP}_{\mathrm{BGK}}\left(x^{*}, z\right)$ denotes the objective of $\operatorname{LP} \mathrm{BGK}_{\mathrm{Bg}}$ by the solution $x^{*}, z$. We now show that

$$
\operatorname{LP}_{\text {Primal }}\left(x^{*}, y^{*}\right)=\operatorname{LP} \operatorname{BGK}\left(x^{*}, z\right)
$$

Since $x^{*}, y^{*}$ is an optimal solution to $L P_{\text {Primal, }}$ and $x^{*}, z$ are a feasible solution to $L P_{B G K}$, the claim will follow. Consider any $S$ and $F \in \mathcal{F}(S)$. By viewing $C_{S}^{F}$ as adding one to the cost of $S$ at each time $t \leqslant C_{S}^{F}$, we have 


$$
\begin{aligned}
& \sum_{S \in \mathcal{S}} \sum_{F \in \mathcal{F}(S)}^{L} C_{S}^{F} y_{S}^{* F} \\
= & \sum_{S \in \mathcal{S}} \sum_{1 \leqslant t \leqslant n} \sum_{F \in \mathcal{F}(S): t \leqslant C_{S}^{F}} y_{S}^{* F}\left(x^{*}, y^{*}\right) \\
= & \left.\sum_{1 \leqslant t \leqslant n} \sum_{S \in \mathcal{S}}\left(1-\sum_{F \in \mathcal{F}(S), t>C_{S}^{F}} y_{S}^{* F}\right) \quad \text { [From constraints }(3)\right] \\
= & \sum_{1 \leqslant t \leqslant n} \sum_{S \in \mathcal{S}}\left(1-z_{S, t}\right) \\
= & \operatorname{LP} P_{B G K}\left(x^{*}, z\right)
\end{aligned}
$$




\subsection{CONCLUSIONS}

We believe that our gap between preemptive and non-preemptive schedules is not tight. In fact, we make the following conjecture:

Conjecture 4.1. Given a preemptive schedule with cost $\mathrm{C}$ then there is a nonpreemptive schedule with cost at most 2C. Further, such a non-preemptive schedule can be found in polynomial time.

It would be also interesting to show if the optimal gap between values of preemptive and non-preemptive schedules depends on parameter $\xi=$ $\min _{S}\{\kappa(S) /|S|\}$. For example, we know if $\xi=1$ then there is no advantage for preemptive schedules because this is equivalent to to Min Latency Set Cover for which preemption does not help.

A slightly different angle is to look at the minimum requirement. In Figure 8 the current knowledge is plotted together with two conjectures. Either the approximation ratios decrease or there is a slight bump and it decreases after the bump. In the figure the "bump" is positioned at minimum requirement 2. This is a little arbitrary: there is no concrete reason to assume it could not be 3 , or 4 for that matter. However, when looking at the analysis of when all requirements are exactly 1 (Min Sum Set Cover), it seems that going from 1 to 2 introduces the obstructions for good approximation ratios.

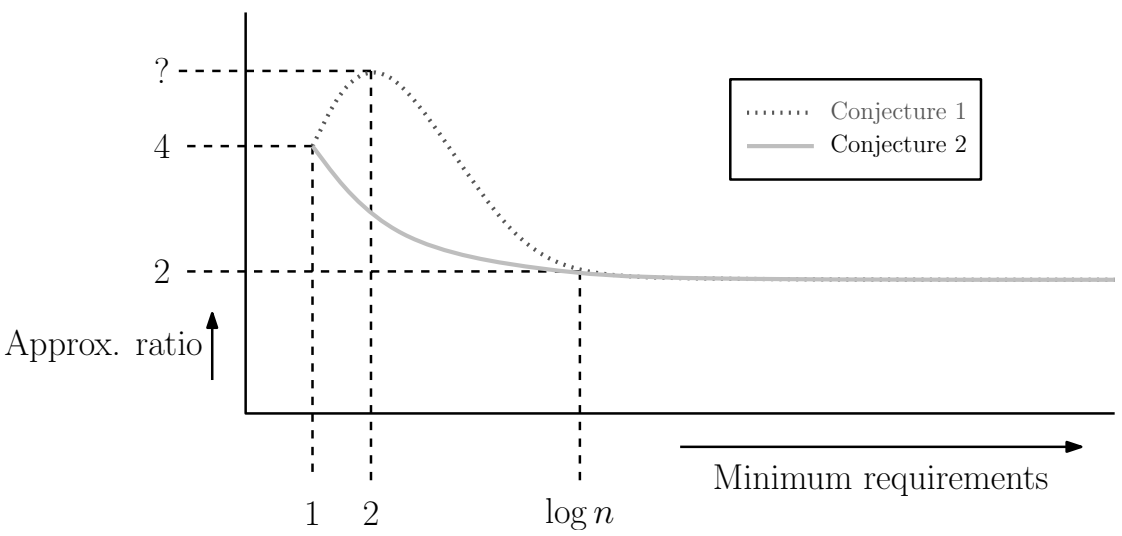

Figure 8: This is a graphical view on what the true approximation guarantee could be. 
We believe a good first step towards a better approximation algorithm for Generalized Min Sum Set Cover is to find a deterministic algorithm that transforms a preemptive schedule to a non-preemptive schedule only losing a constant factor. Such an algorithm should not be a derandomization of our algorithm, but rather a new construction that exploits some structural properties. 


\section{MINIMUM LATENCY SUBMODULAR COVER}

This chapter is based on part of the following paper:

S. Im, V. Nagarajan and R. van der Zwaan, "Minimum Latency Submodular Cover". Accepted at International Colloquium on Automata, Languages and Programming (ICALP) 2012.[63].

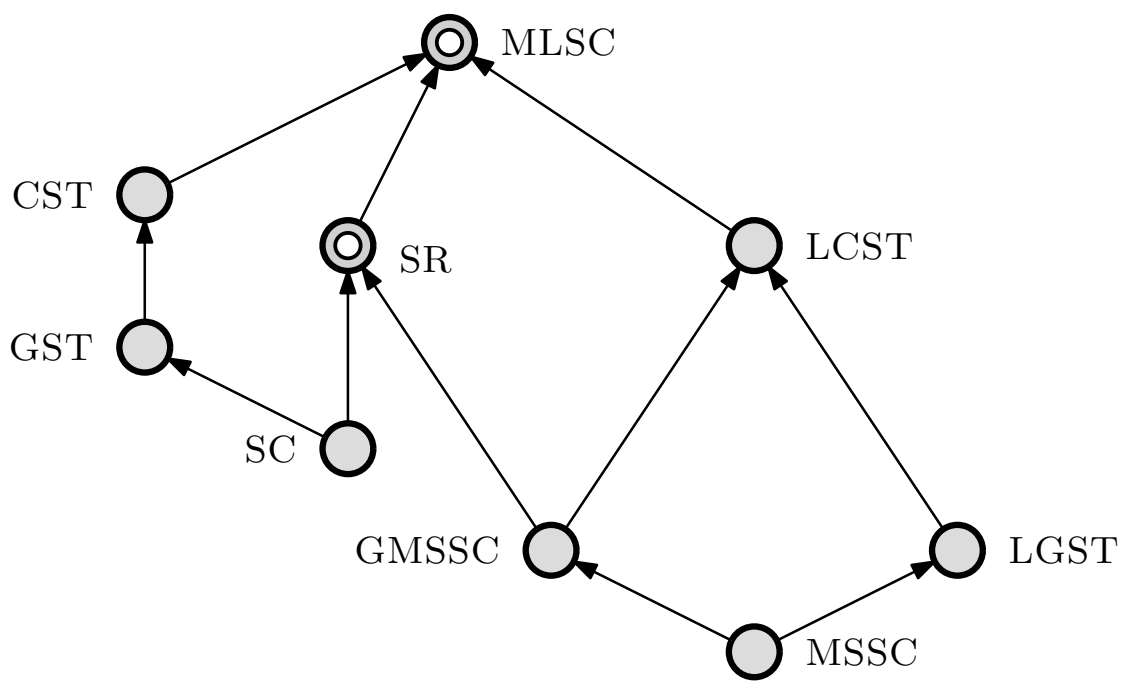




\subsection{INTRODUCTION}

Ordering a set of elements so as to be simultaneously good for several valuations is an important issue in web-search ranking and broadcast scheduling. A formal model for this was introduced by Azar, Gamzu and Yin [6] where they studied the Multiple Intents Re-ranking problem (a.k.a. Generalized Min Sum Set Cover [8]). Subsequently, Azar and Gamzu [5] studied the Submodular Ranking problem where the valuations can be arbitrary (monotone) submodular functions.

In this chapter, we extend both of these models to the setting of general metric switching costs. This allows us to handle additional issues such as:

- Data locality: the time taken to read or transmit data $j$ after data $i$ is $d(i, j)$.

- Context switching: it takes $d(i, j)$ time for a user to parse data $j$ when scheduled after data $i$.

Thus we study the Minimum Latency Submodular Cover problem (MLSC) which is the metric version of submodular ranking [5], and the Latency Covering Steiner Tree problem (LCST) in Chapter 5. We obtain poly-logarithmic approximation guarantees for both problems. We remark that due to a relation to the well-known Group Steiner Tree problem [43], any significant improvement on our results would lead to a similar improvement for Group Steiner Tree.

In a somewhat different direction, we also study the Weighted Stochastic Submodular Ranking problem, where the goal is to adaptively schedule stochastic elements so as to minimize the expected total cover time. We obtain an $\mathrm{O}\left(\log \frac{1}{\epsilon}\right)$-approximation algorithm for this problem, which is known to be best possible even in the deterministic setting [5].

We let $V$ denote the ground set of elements/vertices and $d:\left(\begin{array}{l}V \\ 2\end{array}\right) \rightarrow \mathbb{R}_{+}$ the distance function. We assume that $d$ is symmetric and satisfies the triangle inequality. In addition there is a specified root vertex $r \in V$. There are $m$ monotone submodular functions $f_{1}, \ldots, f_{m}: 2^{V} \rightarrow \mathbb{R}_{+}$representing the valuations of different users. We assume without loss of generalization by truncation that $f_{i}(V)=1$ for all $i \in[m]$. Function $f_{i}$ is said to be covered by set $S \subseteq V$ if $f_{i}(S)=1=f_{i}(V)$. The cover time of function $f_{i}$ in a ordering of $V$, say $\pi$, is the length of the shortest prefix of $\pi$ that has $f_{i}$ value one, i.e.

$\min t: f_{i}(\{v \in V: v$ appears within distance $t$ on $\pi\})=1$. 
The objective in the Minimum Latency Submodular Cover problem (MLSC) is to compute a path originating at $r$ visiting all vertices in $V$ that minimizes the sum of cover times of all functions. We recover the Submodular Ranking problem [5] as the special case when metric $d$ is uniform. A technical parameter that we use to measure performance (this was also used in [5]) is $\epsilon$ which is defined to be the smallest non-zero marginal increase of any function $\left\{f_{i}\right\}_{i=1}^{m}$.

We assume that the submodular functions are given as a value-oracle, so for any submodular function $f$ there is an oracle that returns the value of $f(S)$ in $O(1)$ time. A useful subroutine in our algorithm for MLSC is the Submodular Orienteering problem (SOP) [28] which is the following. The input is a metric $(\mathrm{V}, \mathrm{d})$, a root $r \in \mathrm{V}$, a monotone submodular function $f: 2^{V} \rightarrow \mathbb{R}_{+}$and a length bound $B$. The goal is to find a path originating at $r$ having length at most $B$ that maximizes $f(S)$, where $S \subseteq V$ is the set of vertices visited in the path.

\subsubsection{Our Results}

We start with the general Minimum Latency Submodular Cover problem (MLSC) problem, for which we obtain a poly-logarithmic approximation.

Theorem 5.1. For any constant $\delta>0$, there is an $\mathrm{O}\left(\log \frac{1}{\epsilon} \cdot \log ^{2+\delta}|\mathrm{V}|\right)$-approximation algorithm for the Minimum Latency Submodular Cover problem.

Note that in the special case of Group Steiner Tree, this result is only larger by a factor of $\mathrm{O}\left(\log ^{\delta}|\mathrm{V}|\right)$ than the best known algorithm by Garg et al. which has approximation ratio $\mathrm{O}\left(\log \mathrm{N} \log ^{2}|\mathrm{~V}|\right)$ [43]. In fact our algorithm for MLSC is essentially the algorithm of Azar and Gamzu but we use the algorithm for Submodular Orienteering (SOP) to augment the ordering. Specifically, we show that an $(\rho, \sigma)$-bicriteria approximation algorithm for SOP can used to obtain an $\mathrm{O}\left(\rho \sigma \cdot \log \frac{1}{\epsilon}\right)$-approximation algorithm for MLSC. An $(\rho, \sigma)$ bicriteria guarantee for SOP means that on every instance, the algorithm produces a path of length at most $\sigma$ times the bound and profit at least $\frac{1}{\rho}$ times the optimum that strictly satisfies the length bound. To obtain Theorem 5.I we use an $\left(\mathrm{O}(1), \mathrm{O}\left(\log ^{2+\delta}|\mathrm{V}|\right)\right)$-bicriteria approximation for SOP that follows from [21, 26]. Alternatively, we can use the quasi-polynomial time $\mathrm{O}(\log |\mathrm{V}|)$-approximation for SOP by Chekuri and Pal [28] within our algorithm to obtain: 
Theorem 5.2. There is a quasi-polynomial time $\mathrm{O}\left(\log \frac{1}{\epsilon} \cdot \log |\mathrm{V}|\right)$-approximation algorithm for the Minimum Latency Submodular Cover problem.

Our algorithm for MLSC is an extension of the elegant "adaptive residual updates scheme" of Azar and Gamzu [5] for Submodular Ranking (i.e. uniform metric MLSC). As shown in [5], an interesting aspect of this problem is that the natural greedy algorithm, based on absolute contribution of elements, performs very poorly. Instead they used a modified greedy algorithm that selects one element at a time according to residual coverage. In the MLSC setting of general metrics, our algorithm uses a similar residual coverage function to repeatedly augment the solution. However our augmentations are paths of geometrically increasing lengths, instead of just one element. A crucial point in our algorithm is that the residual coverage functions are always submodular, and hence we can use Submodular Orienteering (SOP) in the augmentation step. We remark that the approach of covering the maximum number of functions within geometrically increasing lengths fails because the residual coverage function here is non-submodular; in fact as noted in [8] this subproblem contains the difficult Dense-k-Subgraph problem (even for generalized min sum set cover with requirement two). We also note that the choice of our (submodular) residual coverage function ultimately draws on the Submodular Ranking algorithm [5].

The analysis in [5] was based on viewing the optimal and approximate solutions as histograms. This approach was first used in this line of work by Feige et al. [4I] for the Min Sum Set Cover problem (see also [1I]). This was also the main framework of analysis in [6] for Generalized Min Sum Set Cover and then for Submodular Ranking [5]. However, these proofs have been increasingly difficult as the problem in consideration adds more generality. Instead we follow a different and more direct approach that is similar to the analysis for the Minimum Latency TSP, see eg. [25, 38]. In Section 5.2 we first give a simpler proof for the algorithm of Azar and Gamzu for Submodular Ranking, which also implies the result in Azar et al. [6]. Then we present our algorithm for MLSC in Section 5.3, which yields Theorems 5.1 and 5.2.

Our second result is for the Weighted Stochastic Submodular Ranking problem. As shown in [46,50], even special cases of this problem have polynomially large adaptivity gap (ratio between the optimal non-adaptive and adaptive solutions). This motivates us to focus on adaptive algorithms, and we obtain the following result in Section 5.4. 
Theorem 5.3. There is an adaptive $\mathrm{O}\left(\log \frac{1}{\epsilon}\right)$-approximation algorithm for the Weighted Stochastic Submodular Ranking problem.

In particular, we show that the most natural stochastic extension of the algorithm from [5] achieves the approximation factor. We remark that the analysis in [5] for deterministic submodular ranking assumed unit costs, whereas Theorem 5.3 holds for the stochastic setting even with nonuniform costs $\left\{\ell_{i}\right\}$.

As mentioned before, our results generalize the results in $[46,83,78]$ which study (some variants of) stochastic set cover problems. Our analysis is arguably simpler and more transparent than [78], which gave the first tight analysis of these problems. We note that the charging scheme used in [78] makes use of "dual prices" and it does not seem directly applicable to general submodular functions.

\subsubsection{Organization}

The remainder of this chapter is organized as follows. In Section 5.2 we give a simple analysis of the approximation algorithm for the Submodular Ranking problem, and in Section 5.3 we extend this to the Minimum Latency Submodular Cover problem. Our final section before the conclusions is Section 5.4.1 where we study the Weighted Stochastic Submodular Ranking problem.

NOTATION We use ALG to denote the cost of the algorithm we consider. For notational simplicity, we let OPT denote the optimal solution itself or the optimal cost depending on the context.

\subsection{SIMPLER ANALYSIS OF THE SUBMODULAR RANK- ING PROBLEM}

In this section, we revisit the Submodular Ranking problem [5]. Recall that the input consists of a ground set $V:=[n]$ of elements and monotone submodular functions $f_{1}, f_{2}, \ldots f_{m}: 2^{[n]} \rightarrow[0,1]$ with $f_{i}(V)=1, \forall i \in[m]$. The goal is to find a complete linear ordering of the elements that minimizes the total cover time of all functions. The cover time $\operatorname{cov}\left(f_{i}\right)$ of $f_{i}$ is defined as the smallest index $t$ such that the function $f_{i}$ has value 1 for the first $t$ 
elements in the ordering. We also say that an element $e$ is scheduled at time $t$ if it is the $t$-th element in the ordering. It is assumed that each function $f_{i}$ satisfies the following property: for any $S \supseteq S^{\prime}$, if $f_{i}(S)-f_{i}\left(S^{\prime}\right)>0$ then it must be the case that $f_{i}(S)-f_{i}\left(S^{\prime}\right) \geqslant \epsilon$, where $\epsilon>0$ is a constant that is uniform for all functions $f_{i}$. This is a useful parameter in describing the performance guarantee.

Azar and Gamzu [5] gave a modified greedy-style algorithm with an approximation factor of $\mathrm{O}\left(\log \frac{1}{\epsilon}\right)$ for Submodular Ranking. Their analysis was histogram-based and fairly involved. In this section, we give an alternate proof of their result. We believe that our analysis is easier and perhaps more intuitive. Our analysis also extends to the more general MLSC problem which we study in the next section. The algorithm ALG-AG from [5] is given below. In the output, $\pi(t)$ denotes the element that appears in the t-th time slot.

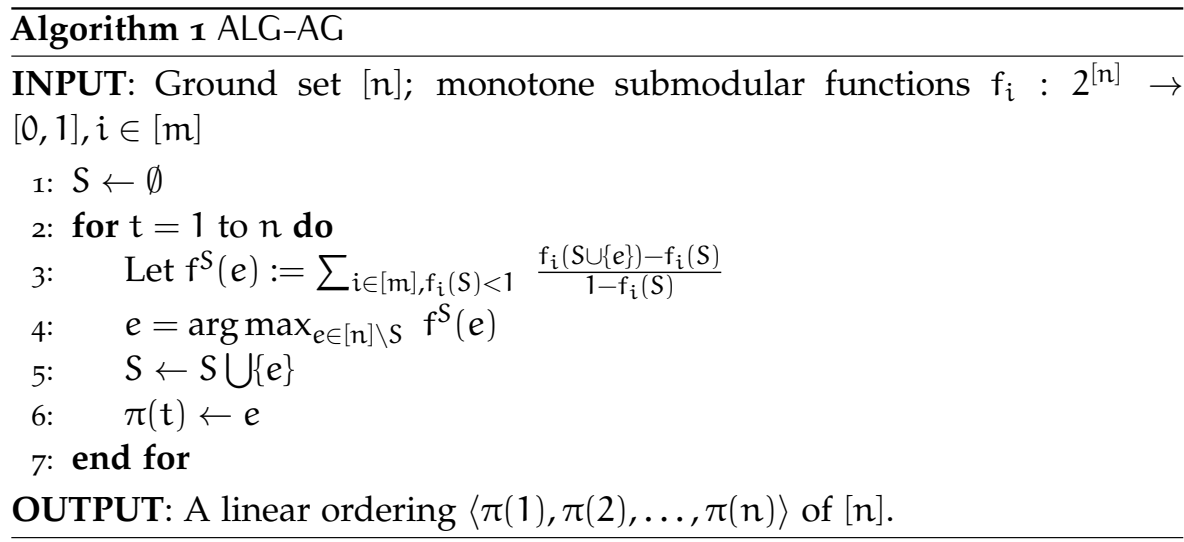

Theorem 5.4 ([5]). The algorithm $A L G-A G$ is an $\mathrm{O}\left(\ln \left(\frac{1}{\epsilon}\right)\right)$-approximation for submodular ranking.

Let $\alpha:=1+\ln \left(\frac{1}{\epsilon}\right)$. To simplify notation, without loss of generality, we assume that $\alpha$ is an integer. Let $R(t)$ denote the set of functions that are not satisfied by ALG-AG earlier than time $t ; R(t)$ includes the functions that are satisfied exactly at time $t$. For notational convenience, we use $i \in R(t)$ interchangeably with $f_{i} \in R(t)$. Analogously, set $R^{*}(t)$ is defined for the optimal solution. Note that $A L G=\sum_{t \geqslant 1}|R(t)|$ and OPT $:=\sum_{t \geqslant 1}\left|R^{*}(t)\right|$. We are interested in the number of unsatisfied functions at times $\left\{8 \alpha 2^{j}\right.$ : $\left.j \in \mathbb{Z}_{+}\right\}$by ALG-AG and the number of unsatisfied functions at times $\left\{2^{j}\right.$ : 
$\left.j \in \mathbb{Z}_{+}\right\}$by the optimal solution. Let $R_{j}:=R\left(8 \alpha 2^{j}\right)$ and $R_{j}^{*}=R^{*}\left(2^{j}\right)$. It is important to note that $R_{j}$ and $R_{j}^{*}$ are concerned with different times. For notational simplicity, we let $R_{-1}:=\emptyset$.

We show the following key lemma. Roughly speaking, it says that the number of unsatisfied functions by ALG-AG diminishes quickly unless it is comparable to the number of unsatisfied functions in OPT.

Lemma 5.5. For any $j \geqslant 0$, we have $\left|R_{j}\right| \leqslant \frac{1}{4}\left|R_{j-1}\right|+\left|R_{j}^{*}\right|$.

Proof. When $j=0$ the lemma trivially holds, therefore consider any time step $t \in\left[8 \alpha 2^{j-1}, 8 \alpha 2^{j}\right)$ for an integer $j \geqslant 1$. Let $S_{t-1}$ denote the set of elements that ALG-AG schedules before time $t$ and let $e_{t}$ denote the element that ALG-AG schedules exactly at time $t$. Let $E_{j}$ denote the set of elements that ALG-AG schedules until time $8 \alpha 2^{j}$. Let $E_{j}^{*}$ denote the set of elements that OPT schedules until time $2^{j}$. Recall that ALG-AG picks $e_{t}$ as an element $e$ that maximizes

$$
f^{S_{t-1}}(e):=\sum_{i \in[m]: f_{i}\left(S_{t-1}\right)<1} \frac{f_{i}\left(S_{t-1} \cup\{e\}\right)-f_{i}\left(S_{t-1}\right)}{1-f_{i}\left(S_{t-1}\right)}
$$

This leads us to the following proposition.

Proposition 5.6. Consider any $j \geqslant 1$ and any time step $t \in\left[8 \alpha 2^{j-1}, 8 \alpha 2^{j}\right)$. Then for any $e \in E_{j}^{*}$, we have $f^{S_{t-1}}\left(e_{t}\right) \geqslant f^{S_{t-1}}(e)$.

Proof. Since ALG-AG has chosen to schedule element $e_{t}$ over all elements $e \in E_{j}^{*} \backslash S_{t-1}$, we know that the claimed inequality holds for any $e \in$ $E_{j}^{*} \backslash S_{t-1}$. Further, the inequality holds for any element $e$ in $S_{t-1}$, since $f^{S_{t-1}}(e)=0$ for such an element $e$.

By taking an average over all elements in $E_{j}^{*}$, we derive

$$
\begin{aligned}
& f^{S_{t-1}}\left(e_{t}\right) \\
\geqslant & \frac{1}{\left|E_{j}^{*}\right|} \sum_{e \in E_{j}^{*}} f^{S_{t-1}}(e) \\
\geqslant & \frac{1}{\left|E_{j}^{*}\right|} \sum_{e \in E_{j}^{*}} \sum_{i \in R_{j} \backslash R_{j}^{*}} \frac{f_{i}\left(S_{t-1} \cup\{e\}\right)-f_{i}\left(S_{t-1}\right)}{1-f_{i}\left(S_{t-1}\right)}
\end{aligned}
$$

Observe that in the second inequality, in the summation we can sum over all functions not only for which $f_{i}\left(S_{t-1}\right)<1$, since $S_{t-1}$ does not cover 
$f_{i}$ for any $i \in R_{j}$ : by definition, $f_{i}$ is not covered before time $8 \alpha 2^{j}$ and $t<8 \alpha 2^{j}$. Due to submodularity of each function $f_{i}$ (more precisely by applying Proposition 2.5 with $A:=E_{j}^{*}$ and $B:=S_{t-1}$ ), we have that

$$
\begin{aligned}
(18) & \geqslant \frac{1}{\left|E_{j}^{*}\right|} \sum_{i \in R_{j} \backslash R_{j}^{*}} \frac{f_{i}\left(S_{t-1} \cup E_{j}^{*}\right)-f_{i}\left(S_{t-1}\right)}{1-f_{i}\left(S_{t-1}\right)} \\
& =\frac{1}{\left|E_{j}^{*}\right|} \sum_{i \in R_{j} \backslash R_{j}^{*}} 1 \geqslant \frac{\left|R_{j}\right|-\left|R_{j}^{*}\right|}{\left|E_{j}^{*}\right|}
\end{aligned}
$$

The equality is due to the fact that for any $i \notin R_{j}^{*}, f_{i}\left(E_{j}^{*}\right)=1$ and each function $f_{i}$ is monotone. Hence:

$$
\begin{aligned}
\sum_{8 \alpha \cdot 2^{j-1} \leqslant \mathrm{t}<8 \alpha \cdot 2^{j}} f^{S_{t-1}\left(e_{t}\right)} & \geqslant \frac{8 \alpha\left(2^{j}-2^{j-1}\right)}{\left|E_{j}^{*}\right|}\left(\left|R_{j}\right|-\left|R_{j}^{*}\right|\right) \\
& =4 \alpha\left(\left|R_{j}\right|-\left|R_{j}^{*}\right|\right)
\end{aligned}
$$

where we used $\left|E_{j}^{*}\right|=2^{j}$. We now upper bound the left-hand-side of (19). To this end, we need the following claim from [5].

Claim 5.7 (Claim 2.3 in [5]). Given a monotone function $\mathrm{f}: 2^{[\mathrm{n}]} \rightarrow[0,1]$ and sets $\emptyset=\mathrm{S}_{0} \subseteq \mathrm{S}_{1} \subseteq \cdots \subseteq \mathrm{S}_{\ell} \subseteq[\mathrm{n}]$, we have (using the convention $0 / 0=0$ )

$$
\sum_{k=1}^{\ell} \frac{f\left(S_{k}\right)-f\left(S_{k-1}\right)}{1-f\left(S_{k-1}\right)} \leqslant 1+\ln \frac{1}{\delta} .
$$

Here $\delta>0$ is such that for any $A \subseteq B$, if $f(B)-f(A)>0$ then $f(B)-f(A) \geqslant \delta$.

Proof. We give a proof for completeness. Order the values in the set $\left\{f\left(S_{k}\right) \mid 0 \leqslant\right.$ $k \leqslant \ell\} \backslash\{1\}$ in increasing order to obtain $\beta_{0}<\beta_{1}<\ldots<\beta_{H}$. By the assumption, we have $\beta_{0}=0,1-\beta_{H} \geqslant \delta$ and $\beta_{h}-\beta_{h-1} \geqslant \delta, \forall h \in[H]$. Note that the desired inequality is reduced to:

$$
\sum_{h=1}^{H} \frac{\beta_{h}-\beta_{h-1}}{1-\beta_{h-1}} \leqslant \ln \frac{1}{\delta}
$$

If $\mathrm{f}\left(\mathrm{S}_{\ell}\right)=1$ then the summation we want to bound has an additional term of $\frac{1-\beta_{H}}{1-\beta_{H}}=1$. 
Knowing that the function $x \rightarrow \frac{1}{1-x}$ is increasing for $x \in[0,1)$, we derive

$$
\begin{aligned}
\sum_{h=1}^{H} \frac{\beta_{h}-\beta_{h-1}}{1-\beta_{h-1}} & =\sum_{h=1}^{H} \int_{x=\beta_{h-1}}^{\beta_{h}} \frac{1}{1-\beta_{h-1}} d x \\
& \leqslant \sum_{h=1}^{H} \int_{x=\beta_{h-1}}^{\beta_{h}} \frac{1}{1-x} d x \\
& =\int_{x=0}^{\beta_{H}} \frac{1}{1-x} d x \\
& =-\ln \left(1-\beta_{H}\right) \leqslant \ln \frac{1}{\delta}
\end{aligned}
$$

This proves the claim.

Note that any function $f_{i}$ not in $R_{j-1}$ does not contribute to the left-handside of (19). This is because such a function $f_{i}$ was already covered before time $8 \alpha 2^{j-1} \leqslant$ t. Further, knowing by Claim 5.7 that each function $f_{i} \in R_{j-1}$ can add at most $\alpha:=1+\ln \frac{1}{\epsilon}$, we can upper bound the left-hand-side of (19) by $\alpha\left|R_{j-1}\right|$. Formally,

$$
\begin{aligned}
& \sum_{8 \alpha \cdot 2^{j-1}<t \leqslant 8 \alpha \cdot 2^{j}} f^{S_{t-1}\left(e_{t}\right)} \\
= & \sum_{8 \alpha \cdot 2^{j-1}<t \leqslant 8 \alpha \cdot 2^{j}} \sum_{i \in R_{j-1}: f_{i}\left(S_{t-1}\right)<1} \frac{f_{i}\left(S_{t-1} \cup\left\{e_{t}\right\}\right)-f_{i}\left(S_{t-1}\right)}{1-f_{i}\left(S_{t-1}\right)} \\
\leqslant & \sum_{i \in R_{j-1}} \sum_{t \geqslant 1: f_{i}\left(S_{t-1}\right)<1} \frac{f_{i}\left(S_{t-1} \cup\left\{e_{t}\right\}\right)-f_{i}\left(S_{t-1}\right)}{1-f_{i}\left(S_{t-1}\right)} \\
\leqslant & \alpha\left|R_{j-1}\right|
\end{aligned}
$$

From (19) and (21) we obtain

$$
4 \alpha\left(\left|R_{j}\right|-\left|R_{j}^{*}\right|\right) \leqslant \alpha\left|R_{j-1}\right|
$$

which simplifies to the desired inequality in Lemma 5.5.

Now we can prove Theorem 5.4 using Lemma 5.5. 
Proof of Theorem 5.4 .

$$
\begin{aligned}
A L G= & \sum_{j \geqslant 0} \sum_{8 \alpha 2^{j} \leqslant t<8 \alpha 2^{j+1}}|R(t)|+\sum_{1 \leqslant t<8 \alpha}|R(t)| \\
\leqslant & \sum_{j \geqslant 0} 8 \alpha\left(2^{j+1}-2^{j}\right)\left|R_{j}\right|+8 \alpha O P T \\
& {[|R(t)| \text { is non-increasing, and } \forall t \geqslant 1,|R(t)| \leqslant m \leqslant O P T] } \\
= & 8 \alpha \sum_{j \geqslant 0} 2^{j+1}\left(\left|R_{j}\right|-\frac{1}{4}\left|R_{j-1}\right|\right)+8 \alpha O P T \\
\leqslant & 8 \alpha \sum_{j \geqslant 0} 2^{j+1}\left|R_{j}^{*}\right|+8 \alpha O P T \quad[\text { By Lemma 5.5] } \\
\leqslant & 8 \alpha \sum_{j \geqslant 1} 4 \sum_{2^{j-1} \leqslant t<2^{j}}\left|R^{*}(t)\right|+16 \alpha\left|R_{0}^{*}\right|+8 \alpha O P T \\
\leqslant & 32 \alpha O P T+24 \alpha O P T \quad\left[\text { Since }\left|R^{*}(t)\right|\right. \text { is non-increasing] }
\end{aligned}
$$

Thus we obtain ALG $\leqslant 56 \alpha \mathrm{OPT}$, which proves Theorem 5.4.

\subsection{MINIMUM LATENCY SUBMODULAR COVER}

Recall that in the Minimum Latency Submodular Cover problem (MLSC), we are given a metric $(\mathrm{V}, \mathrm{d})$ with root $r \in \mathrm{V}$ and $\mathrm{m}$ monotone submodular functions $f_{1}, f_{2}, \ldots, f_{m}: 2^{V} \rightarrow[0,1]$. Each function $f_{i}$ satisfies the following properties: $(1) f_{i}(V)=1$ and (2) for any $S^{\prime} \supset S$, if $f_{i}\left(S^{\prime}\right)-f_{i}(S)>0$, then it must be the case that $f_{i}\left(S^{\prime}\right)-f_{i}(S) \geqslant \epsilon$, where $\epsilon>0$ is a value that is uniform for all functions. Without loss of generality, we assume that $\mathrm{d}(\cdot, \cdot)$ is an integer. The goal is to find a path starting at $r$ that minimizes the total cover time of all functions.

As mentioned earlier, our algorithm for MLSC uses as a subroutine an algorithm for the Submodular Orienteering problem (SOP). Here given metric $(\mathrm{V}, \mathrm{d})$, root $\mathrm{r}$, monotone submodular function $\mathrm{g}: 2^{\mathrm{V}} \rightarrow \mathbb{R}_{+}$and bound $B$, the goal is to compute a path $P$ originating at $r$ having length at most $B$ that maximizes $g(V(P))$ where $V(P)$ denotes the set of vertices covered by $P$. We assume a $(\rho, \sigma)$-bicriteria approximation algorithm ALG-SOP for SOP, i.e. on any SOP instance returns a path $P$ of length at most $\sigma \cdot B$ and $g(V(P)) \geqslant O P T / \rho$. We recall the following known results on SOP. 
Theorem $5.8([21])$. For any constant $\delta>0$ there is a polynomial time $(\mathrm{O}(1)$, $\left.\mathrm{O}\left(\log ^{2+\delta}|\mathrm{V}|\right)\right)$ bicriteria approximation algorithm for the Submodular Orienteering problem.

Theorem 5.9 ([28]). There is a quasi-polynomial time $\mathrm{O}(\log |\mathrm{V}|)$ approximation algorithm for the Submodular Orienteering problem.

We now describe our algorithm ALG-LSCP for MLSC that uses the $(\rho, \sigma)$ bicriteria approximation algorithm ALG-SOP. Here $\alpha=1+\ln \frac{1}{\epsilon}$. Note the difference from the submodular ranking algorithm [5]: here each augmentation is a path possibly covering several vertices. Despite the similarity of ALG-LSCP to the min-latency TSP type algorithms [25, 38] an important difference is that we do not try to directly maximize the number of covered functions in each augmentation: as noted before this subproblem is at least as hard as dense-k-subgraph, for which the best approximation ratio known is only polynomial [16]. Instead we maximize in each step some proxy residual coverage function $f^{S}$ that suffices to eventually cover all functions quickly. This function is a natural extension of the singleelement coverage values used in ALG-AG [5]. It is important to note that in Line $(4), f^{S}(\cdot)$ is defined adaptively based on the current set $S$ of visited vertices in each iteration. Moreover, since each function $f_{i}$ is monotone and submodular, so is $f^{S}$ for any $S \subseteq V$. In Line (6), $\pi \cdot P$ implies the concatenation of $\pi$ and P.

We prove the following theorem, which implies Theorem 5.1 and 5.2.

Theorem 5.10. $A L G-L S C P$ is an $\mathrm{O}(\alpha \rho \sigma)$-approximation algorithm for Minimum Latency Submodular Cover.

We now analyze ALG-LSCP. We say that the algorithm is in the $j$-th phase, when the variable $k$ of the for loop in Line (2) has value $j$. Observe that the final solution visits all vertices that are added in the $j$-th phase within time $16 \alpha \rho 2^{j}$. This can be easily shown as follows: the final solution is a concatenation of the paths that were found in Line (5). Since all these paths are stitched at the root $r$, the length of $\pi$ at the end of phase $j$ is at most $\sum_{k=1}^{j} 2 \cdot 4 \alpha \rho \cdot \sigma 2^{k} \leqslant 16 \alpha \rho \sigma \cdot 2^{j}$. The following proposition easily follows.

Proposition 5.11. Any vertex $v$ added to $S$ in the $\mathrm{j}$-th phase is visited by $\pi$ within $16 \alpha \rho \sigma \cdot 2^{j}$.

Let $R(t)$ denote the set of (indices of) the functions that are not covered by ALG-LSCP earlier than time $t ; R(t)$ includes the functions that are covered 


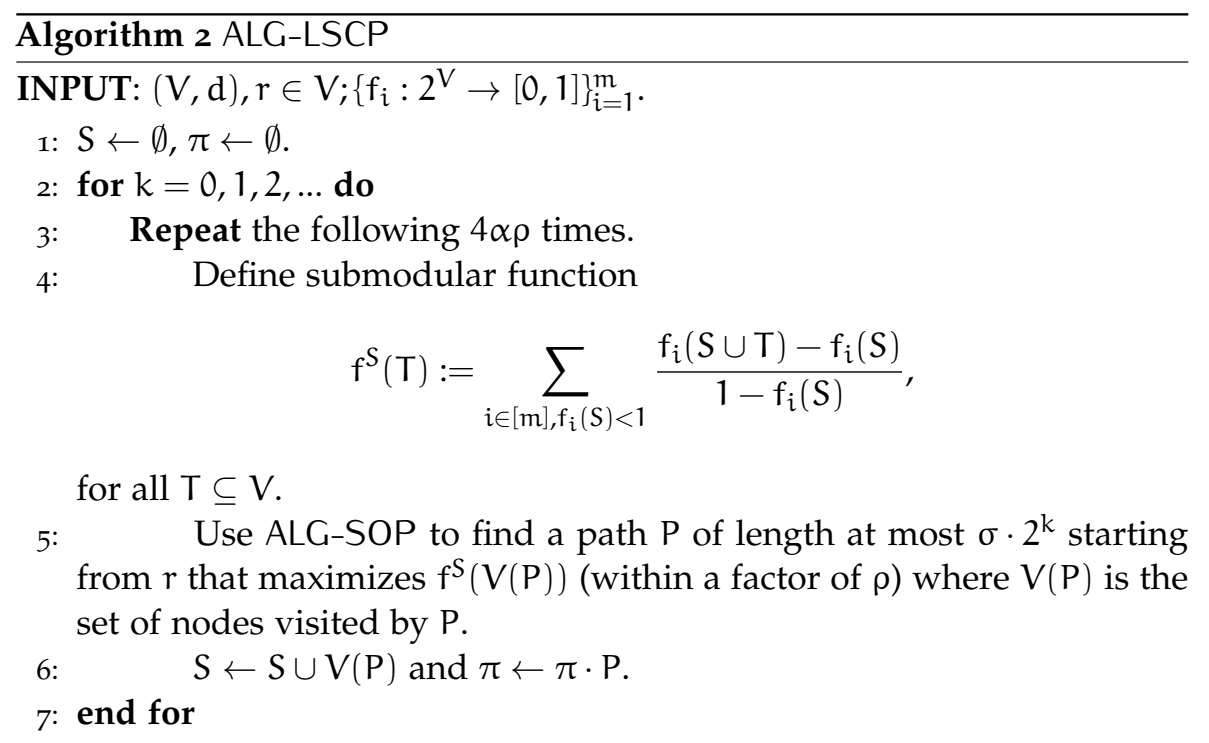

OUTPUT: Output solution $\pi$.

exactly at time $t$ as well. We interchangeably use $i \in R(t)$ from $f_{i} \in R(t)$ for notational simplicity. Let $R_{j}:=R_{j}\left(16 \alpha \rho \sigma 2^{j}\right)$. Similarly, we let $R^{*}(t)$ denote the set of functions that are not covered by OPT earlier than time $t$ and let $R_{j}^{*}=R^{*}\left(2^{j}\right)$. For notational convenience, we let $R_{-1}:=\emptyset$.

We show the following key lemma. It shows that the number of uncovered functions by ALG-LSCP must decrease fast as $j$ grows, unless the corresponding number by the optimal solution is comparable.

Lemma 5.12. Consider any $j \geqslant 0$. Then we have $\left|R_{j}\right| \leqslant \frac{1}{4}\left|R_{j-1}\right|+\left|R_{j}^{*}\right|$.

Proof. The lemma trivially holds when $j=0$, hence consider any fixed phase $j \geqslant 1$. Let $S_{0}$ denote the set of vertices that were added to $S$ up to the end of phase $j-1$. Let $H=4 \alpha \rho$ and $T_{1}, T_{2}, \ldots, T_{H}$ be the sets of vertices that were added in Line (6) in the $j$-th phase. Let $S_{h}=S_{0} \cup T_{1} \cup T_{2} \cup \ldots \cup T_{h}$, $\forall 1 \leqslant h \leqslant H$. We prove Lemma 5.12 by lower and upper bounding the quantity

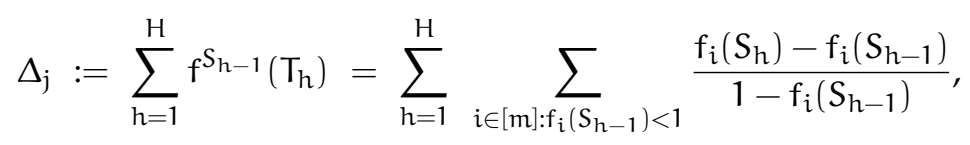

which is roughly the total amount of "residual requirement" that is covered by the algorithm in phase $j$. 
We first lower bound $\Delta_{j}$. Let $\mathrm{T}^{*}$ denote the set of vertices that OPT visited within time $2^{j}$. Observe that in Line (5), ALG-LSCP could have visited all nodes in $\mathrm{T}^{*}$ by choosing $\mathrm{P}$ as the prefix of length $2^{\mathrm{j}}$ of OPT. Via the approximation guarantee of ALG-SOP, we obtain

Proposition 5.13. For any $h \in[H]$ and $i \in R_{j}$, we have $f^{S_{h-1}}\left(S_{h}\right) \geqslant \frac{1}{\rho}$. $f^{S_{h-1}\left(T^{*}\right) .}$

We restrict our concern to the functions in $R_{j} \backslash R_{j}^{*}$. Observe that for any $i \in R_{j}$ and $h \in[H], f_{i}\left(S_{h-1}\right)<1$ and that for any $i \notin R_{j}^{*}, f_{i}\left(T^{*}\right)=1$. Hence by summing the inequality in the above proposition over all functions $f_{i}$ in $R_{j} \backslash R_{j}^{*}$, we have

$$
\begin{aligned}
\Delta_{j} & \geqslant \frac{1}{\rho} \sum_{h=1}^{H} f^{S_{h-1}\left(T^{*}\right)} \\
& \geqslant \frac{1}{\rho} \sum_{h=1}^{H} \sum_{i \in R_{j} \backslash R_{j}^{*}} \frac{f_{i}\left(T^{*} \cup S_{h-1}\right)-f_{i}\left(S_{h-1}\right)}{1-f_{i}\left(S_{h-1}\right)} \\
& \geqslant \frac{1}{\rho} \sum_{h=1}^{H} \sum_{i \in R_{j} \backslash R_{j}^{*}} 1 \\
& \geqslant \frac{H}{\rho}\left(\left|R_{j}\right|-\left|R_{j}^{*}\right|\right)=4 \alpha\left(\left|R_{j}\right|-\left|R_{j}^{*}\right|\right)
\end{aligned}
$$

We now upper bound $\Delta_{j}$. Note by definition that for any $i \notin R_{j-1}$, $f_{i}\left(S_{0}\right)=1$ and therefore $f_{i}$ does not contribute to $\Delta_{j}$. For any $i \in R_{j-1}$, the total contribution of $f_{i}$ to $\Delta_{j}$ is at most $\alpha$ by Claim 5.7. Hence it follows that

$$
\Delta_{j} \leqslant \alpha\left|R_{j-1}\right|
$$

Combining (22) and (23) completes the proof of Lemma 5.12.

We prove Theorem 5.10 exactly as we proved Theorem 5.4 in the previous section using Lemma $5 \cdot 5$. 
Proof of Theorem 5.10.

$$
\begin{aligned}
= & \sum_{j \geqslant 0}^{\text {ALG }} \sum_{16 \alpha \rho \sigma 2^{j} \leqslant \mathrm{t}<16 \alpha \rho \alpha^{j+1}}|\mathrm{R}(\mathrm{t})|+\sum_{0 \leqslant \mathrm{t}<16 \alpha \rho \sigma}|\mathrm{R}(\mathrm{t})| \\
\leqslant & \sum_{j \geqslant 0} 16 \alpha \rho \sigma\left(2^{j+1}-2^{j}\right)\left|R_{j}\right|+16 \alpha \rho \sigma \mathrm{OPT} \\
& {[|\mathrm{R}(\mathrm{t})| \text { is non-increasing, and } \forall \mathrm{t} \geqslant 0,|\mathrm{R}(\mathrm{t})| \leqslant \mathrm{m} \leqslant \mathrm{OPT}] } \\
= & 16 \alpha \rho \sigma \sum_{j \geqslant 0} 2^{j+1}\left(\left|R_{j}\right|-\frac{1}{4}\left|\mathrm{R}_{\mathrm{j}-1}\right|\right)+16 \alpha \rho \sigma \mathrm{OPT} \\
\leqslant & 16 \alpha \rho \sigma \sum_{j \geqslant 0} 2^{j+1}\left|\mathrm{R}_{\mathrm{j}}^{*}\right|+16 \alpha \rho \sigma \mathrm{OPT} \quad[\text { Lemma 5.12] } \\
\leqslant & 16 \alpha \rho \sigma \sum_{j \geqslant 1} 4\left(\sum_{2^{j-1} \leqslant \mathrm{t}<2^{j}}\left|\mathrm{R}^{*}(\mathrm{t})\right|\right)+32 \alpha \rho \sigma\left|\mathrm{R}_{0}^{*}\right|+16 \alpha \rho \sigma \mathrm{OPT} \\
\leqslant & 56 \alpha \rho \sigma \mathrm{OPT}+48 \alpha \rho \sigma \mathrm{OPT} \quad\left[\left|\mathrm{R}^{*}(\mathrm{t})\right|\right. \text { is non-increasing] }
\end{aligned}
$$

\subsection{WEIGHTED STOCHASTIC SUBMODULAR RANK- ING}

In this section, we study the Weighted Stochastic Submodular Ranking problem. The input consists of a set $\mathcal{A}=\left\{X_{1}, \ldots, X_{n}\right\}$ of $n$ independent random variables (stochastic elements, each over domain $\Delta$ ) with integer lengths $\left\{\ell_{i}\right\}_{i=1}^{n}$ (deterministic), and $m$ monotone submodular functions $f_{1}, \ldots, f_{m}: 2^{\Delta} \rightarrow[0,1]$ on groundset $\Delta$. We are also given the distribution (over $\Delta$ ) of each stochastic element $\left\{X_{i}\right\}_{i=1}^{n}$. The realization $x_{i} \in \Delta$ of the random variable $X_{i}$ is known immediately after scheduling $X_{i}$. Here, $X_{i}$ (and $x_{i}$ ) requires $\ell_{i}$ units of time to be scheduled; recall that in the non-stochastic submodular ranking, elements were assumed to have unit sizes. A feasible solution/policy is an adaptive ordering of $\mathcal{A}$, represented naturally by a decision tree with branches corresponding to the realization of the stochastic elements. We use $\langle\pi(1), \ldots, \pi(n)\rangle$ to denote this ordering, where each $\pi(l)$ is a random variable denoting the index of the l-th scheduled element. 
Note that we did not state how the distribution is given. The only assumption that is needed for the algorithm to run in polynomial time is that we can sample according to the distribution in polynomial time.

The cover time $\operatorname{cov}\left(f_{i}\right)$ of $f_{i}$ is defined as the earliest time $t$ such that $f_{i}$ has value one for the realization of the elements completely scheduled within time $t$. More formally, $\operatorname{cov}\left(f_{i}\right)$ is the earliest time $t$ such that $f_{i}\left(\left\{x_{\pi(1)}, \ldots, x_{\pi\left(k_{t}\right)}\right\}\right)$ is equal to 1 where $k_{t}$ is the maximum integer such that $\ell_{\pi(1)}+\ell_{\pi(2)}+\ldots+$ $\ell_{\pi\left(k_{t}\right)} \leqslant t$. If the function value never reaches one (due to the stochastic nature of elements) then $\operatorname{cov}\left(f_{i}\right)=\ell_{1}+\ell_{2}+\ldots+\ell_{n}$. Note that the cover time is a random value. The goal is to find a policy that (approximately) minimizes the expected total cover time $\mathbb{E}\left[\sum_{i \in[m]} \operatorname{cov}\left(f_{i}\right)\right]$. As in the previous sections, each function $f_{i}$ satisfies the following property: for any $S \supseteq S^{\prime}$, if $f_{i}(S)-f_{i}\left(S^{\prime}\right)>0$ then it must be the case that $f_{i}(S)-f_{i}\left(S^{\prime}\right) \geqslant \epsilon$, where $\epsilon>0$ is a uniform value for all functions $f_{i}$.

Our stochastic extension captures many interesting applications.

Adaptive Set Cover: We are given as input a ground set $\Delta$, and a collection $\mathcal{S} \subseteq 2^{\Delta}$ of (non-stochastic) sets. There are stochastic elements $\left\{X_{i}: i \in[n]\right\}$, each defined over $\Delta$, and having costs. The goal is to give an adaptive policy that hits all sets in $\mathcal{S}$ using the minimum cost of (stochastic) elements. This problem was studied in $[46,83,78]$. The problem can be shown as an instance of stochastic SR: There is a single monotone submodular function $f_{1}(A):=\frac{1}{|\mathcal{S}|} \sum_{S \in \mathcal{S}} \min \{1,|A \cap S|\}$ and $\epsilon=1 /|\mathcal{S}|$.

Shared Filter Evaluation: This problem was introduced by [83], and the result was improved to an essentially optimal solution in [78]. In this problem, there is a collection of independent filters $X_{1}, X_{2}, \ldots ., X_{n}$, each gets evaluated either to True or False. For each filter $i \in[n]$, we are given the "selectivity" $p_{i}=\operatorname{Pr}\left[X_{i}\right.$ is true $]$ and the cost $\ell_{i}$ of running the filter. We are also given a collection $\mathcal{Q}$ of queries, where each query $Q_{j}$ is a conjunction of a subset of queries. We would like to determine each query in $Q$ to be True or False by (adaptively) testing filters of the minimum cost. In order to cast this problem as stochastic $S R$, we use $\Delta=\bigcup_{i=1}^{n}\left\{t_{i}, f_{i}\right\}$; for each $i \in[n], X_{i}=t_{i}$ with probability $p_{i}$, and $X_{i}=f_{i}$ with the remaining probability $1-p_{i}$. We create one monotone submodular function:

$$
f_{1}(A):=\frac{\sum_{Q_{j} \in \mathcal{Q}} \min \left\{1,\left|A \cap\left\{f_{i}: i \in Q_{j}\right\}\right|+\frac{1}{\left|Q_{j}\right|} \cdot\left|A \cap\left\{t_{i}: i \in Q_{j}\right\}\right|\right\}}{|Q|}
$$


We note that the Shared Filter Evaluation problem can be studied for a latency type of objective also. In this case, for each query $Q_{j} \in 2$, we create a separate submodular function:

$$
f_{j}(A):=\min \left\{1,\left|A \cap\left\{f_{i}: i \in Q_{j}\right\}\right|+\frac{1}{\left|Q_{j}\right|} \cdot\left|A \cap\left\{t_{i}: i \in Q_{j}\right\}\right|\right\}
$$

In this case, the stochastic SR problem corresponds precisely to filter evaluation that minimizes the average time to answer queries in $Q$.

Stochastic Generalized Min Sum Set Cover: We are given as input a ground set $\Delta$, and a collection $\mathcal{S} \subseteq 2^{\Delta}$ of (non-stochastic) sets with requirement $k(S)$ for each $S \in \mathcal{S}$. There are stochastic elements $\left\{X_{i}: i \in[n]\right\}$, each defined over $\Delta$. Set $S \in \mathcal{S}$ is said to be completed when at least $k(S)$ elements from $S$ have been scheduled. The goal is to find an adaptive ordering of $[n]$ so as to minimize the expected total completion time. This can be reduced to stochastic $S R$ by defining function $f^{S}(A):=\min \{1,|A \cap S| / k(S)\}$ for each $S \in \mathcal{S}$; here $\epsilon=1 / k_{\max }$ where $k_{\max }$ denotes the maximum requirement.

We consider adaptive policies: this chooses at each time $\ell_{\pi(1)}+\ell_{\pi(2)}+$ $\ldots+\ell_{\pi(k-1)}$, the element

$$
\mathrm{X}_{\pi(\mathrm{k})} \in \mathcal{A} \backslash\left\{\mathrm{X}_{\pi(1)}, \mathrm{X}_{\pi(2)}, \mathrm{X}_{\pi(3)}, \ldots, \mathrm{X}_{\pi(\mathrm{k}-1)}\right\}
$$

after observing the realizations $x_{\pi(1)}, \ldots, x_{\pi(k-1)}$. So it can be described as a decision tree. Our main result is an $\mathrm{O}\left(\log \frac{1}{\epsilon}\right)$-approximate adaptive policy. This result is again inspired by our simpler analysis of [5].

By definition, the Weighted Stochastic Submodular Ranking problem generalizes its deterministic counterpart where elements have non-uniform sizes. We note that [5] studied the deterministic submodular ranking only when all elements have unit sizes. For the Stochastic Generalized Min Sum Set Cover problem, our result implies an $\mathrm{O}\left(\log \mathrm{k}_{\max }\right)$-approximation to adaptive policies. However for non-adaptive policies, one can obtain a better $\mathrm{O}(1)$-approximation algorithm by combining the Sample Average Approximation (SAA) method [73, 24] with $\mathrm{O}(1)$-approximations known for the non-stochastic version $[8,98]$.

\subsubsection{Algorithm and Analysis}

To formally describe our algorithm, we quickly define the probability spaces we are concerned with. We use $\Omega=\Delta^{n}$ to denote the outcome space of 
$\mathcal{A}$. We use the same notation $\Omega$ to denote the probability space induced by this outcome space. For any $S \subseteq \mathcal{A}$ and its realization $s$, let $\Omega(s)$ denote the outcome subspace that conforms to $\mathrm{s}$. We can naturally define the probability space defined by $\Omega(s)$ as follows: The probability that $w \in \Omega(s)$ occurs is $\operatorname{Pr}_{\Omega}[w] / \operatorname{Pr}_{\Omega}[\Omega(s)]$. We also use $\Omega(s)$ to denote this probability space.

The main algorithm is given below and is a natural extension of the algorithm by Azar and Gamzu [5]. In the algorithm, Let $\alpha:=1+\ln \left(\frac{1}{\epsilon}\right)$. In the output, $\pi(l)$ denotes the lth element (random variable) in $\mathcal{A}$ that appears in the output.

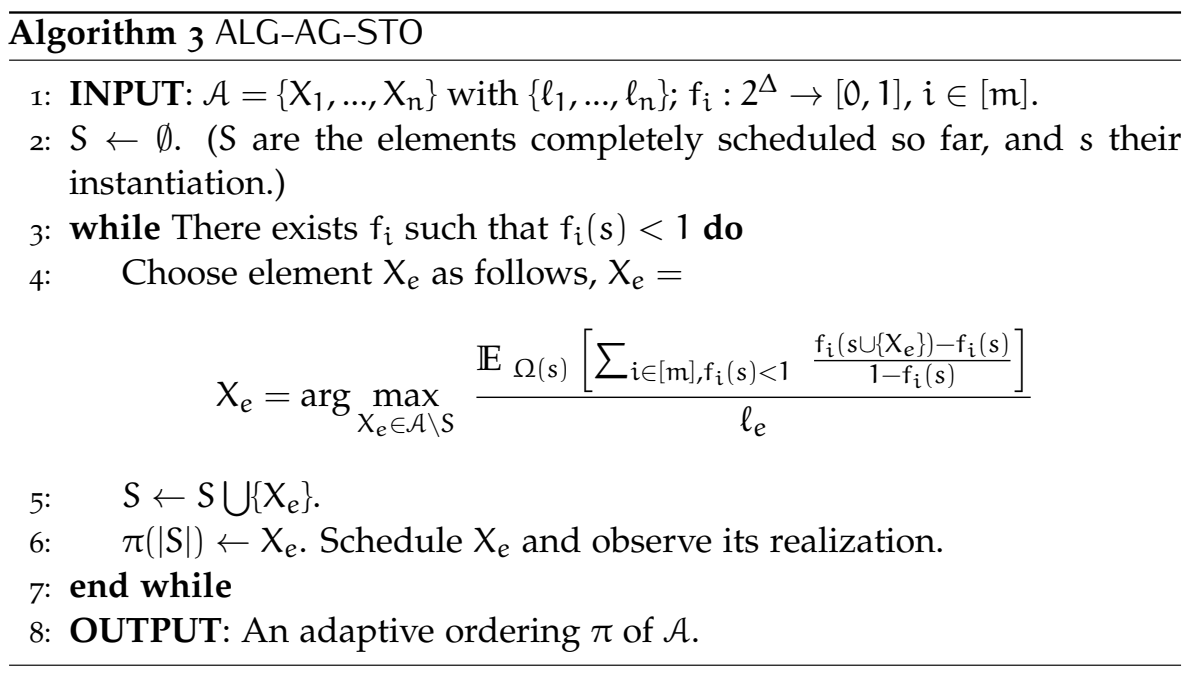

Observe that taking expectation over $\Omega(s)$ in Step 4 is the same as expectation over the distribution of $X_{e}$ since $X_{e} \notin S$ and the elements are independent. This value can be computed almost exactly by sampling, and the small error incurred is disregarded in the following analysis. Also note that this algorithm implicitly defines a decision tree.

Theorem 5.14. ALG-AG-STO is an $\mathrm{O}\left(\ln \left(\frac{1}{\epsilon}\right)\right)$-approximation algorithm for stochastic submodular ranking.

To simplify notation, without loss of generality, we assume that $\alpha$ is an integer. Let $R(t)$ denote the (random) set of functions that are not satisfied by ALG-AG-STO before time $t$. Note that the set $R(t)$ includes the functions that are satisfied exactly at time $t$. Analogously, the set $R^{*}(t)$ is defined for the optimal (in expectation) decision tree. For notational convenience, we 
use $i \in R(t)$ interchangeably with $f_{i} \in R(t)$. Let $C(t):=\left\{f_{1}, \ldots, f_{m}\right\} \backslash R(t)$ and $C^{*}(t):=\left\{f_{1}, \ldots, f_{m}\right\} \backslash R^{*}(t)$. Note that all the sets $C(\cdot), C^{*}(\cdot), R(\cdot), R^{*}(\cdot)$ are stochastic. We have that $A L G=\sum_{t \in[n]}|R(t)|$ and OPT $:=\sum_{t \in[n]}\left|R^{*}(t)\right|$ and hence $A L G$ and OPT are stochastic quantities. We show that $\mathbb{E}[A L G]=$ $\mathrm{O}(\alpha) \cdot \mathbb{E}[\mathrm{OPT}]$ which suffices to prove Theorem 5.14.

We are interested in the number of unsatisfied functions at times $\left\{8 \alpha 2^{j}\right.$ : $\left.j \in \mathbb{Z}_{+}\right\}$by ALG-AG-STO and the number of unsatisfied functions at times $\left\{2^{j}: j \in \mathbb{Z}_{+}\right\}$by the optimal solution. Let $R_{j}:=R\left(8 \alpha 2^{j}\right)$ and $R_{j}^{*}=R^{*}\left(2^{j}\right)$. It is important to note that $R_{j}$ and $R_{j}^{*}$ are concerned with different times, and they are stochastic. For notational simplicity, we let $R_{-1}:=\emptyset$.

We show the following key lemma. Once we get this lemma, we can prove Theorem 5.14 similar to the proof of Theorem 5.4.

Lemma 5.15. For any $j \geqslant 0$, we have $\mathbb{E}\left[\left|R_{j}\right|\right] \leqslant \frac{1}{4} \mathbb{E}\left[\left|R_{j-1}\right|\right]+\mathbb{E}\left[\left|R_{j}^{*}\right|\right]$.

Proof. The lemma trivially holds for $j=0$, so we consider any $j \geqslant 1$. For any $t \geqslant 1$, we use $s_{t-1}$ to denote the set of elements completely scheduled by ALG-AG-STO by time $t-1$ along with their instantiations; clearly this is a random variable. Also, for $t \geqslant 1$ let $\sigma(t) \in[n]$ denote the (random) index of the element being scheduled during time slot $(t-1, t] .^{1}$ Note that $s_{t-1}$ determines $\sigma(t)$ precisely, but not the instantiation of $X_{\sigma(t)}$.

Let $\mathrm{E}_{j}^{*} \subseteq \mathcal{A}$ be the (stochastic) set of elements that is completely scheduled by the optimal policy within time $2^{j}$. For a certain stochastic set (or elements) $S$, we denote its realization under an outcome $w$ as $S(w)$. For example, $X_{i}(w) \in \Delta$ is the realization of element $X_{i}$ for outcome $w$; and $E_{j}^{*}(w)$ is the set of first $2^{j}$ elements completely scheduled by OPT (under $w)$ along with their realizations.

For any time $t$ and corresponding outcome $s_{t-1}$, define a set function:

$$
f^{s_{t-1}}(D):=\sum_{i \in[m], f_{i}\left(s_{t-1}\right)<1} \frac{f_{i}\left(s_{t-1} \cup D\right)-f_{i}\left(s_{t-1}\right)}{1-f_{i}\left(s_{t-1}\right)}, \quad \forall D \subseteq \Delta .
$$

We also use $f_{i}^{s_{t-1}}(D)$ to denote the term inside the above summation.

The function $f^{s_{t-1}}: 2^{\Delta} \rightarrow \mathbb{R}_{+}$is monotone and submodular since it is a summation of monotone and submodular functions. We also define

$$
\mathrm{F}^{\mathrm{s}_{\mathrm{t}-1}}\left(\mathrm{X}_{e}\right):=\mathbb{E}_{w \leftarrow \Omega\left(s_{\mathrm{t}-1}\right)}\left[\mathrm{f}^{\mathrm{s}_{\mathrm{t}-1}}\left(\mathrm{X}_{\mathrm{e}}(w)\right)\right], \quad \forall \mathrm{X}_{e} \in \mathcal{A} .
$$

Observe that this is zero for elements $X_{e} \in s_{t-1}$.

I Since elements have different sizes, note that $\sigma(t)$ is different from $\pi(t)$ which is the $t$-th element scheduled by ALG-AG-STO. 
Proposition 5.16. Consider any time $\mathrm{t} \in[\mathrm{n}]$ and outcome $\mathrm{s}_{\mathrm{t}-1}$. Note that $\mathrm{s}_{\mathrm{t}-1}$ determines $\sigma(\mathrm{t})$. Then:

$$
\frac{1}{\ell_{\sigma(t)}} \cdot F^{s_{t-1}}\left(X_{\sigma(t)}\right) \geqslant \frac{1}{\ell_{i}} \cdot F^{s_{t-1}}\left(X_{i}\right), \quad \forall X_{i} \in \mathcal{A}
$$

Proof. At some time $\mathrm{t}^{\prime} \leqslant \mathrm{t}$ (right after $\mathrm{s}_{\mathrm{t}-1}$ is observed) ALG-AG-STO chose to schedule element $X_{\sigma(t)}$ over all elements $X_{i} \in \mathcal{A} \backslash s_{t-1}$. By the greedy rule we know that the claimed inequality holds for any $X_{i} \in \mathcal{A} \backslash s_{t-1}$. Furthermore, the inequality holds for any element $X_{i} \in s_{t-1}$, since $F^{s_{t-1}}\left(X_{i}\right)=0$ for such an element.

We now define the expected gain by ALG-AG-STO in step $t$ as:

$$
\mathrm{G}_{\mathrm{t}}:=\mathbb{E}_{\mathrm{s}_{\mathrm{t}-1}}\left[\frac{1}{\ell_{\sigma(\mathrm{t})}} \mathrm{F}^{s_{\mathrm{t}-1}\left(\mathrm{X}_{\sigma(\mathrm{t})}\right)}\right] .
$$

And the expected total gain:

$$
\Delta_{j}:=\sum_{t=8 \alpha 2^{j-1}}^{8 \alpha 2^{j}} G_{t}
$$

We complete the proof of Lemma 5.15 by upper and lower bounding $\Delta_{j}$.

UPPER BOUND FOR $\Delta_{j}$. Fix any outcome $w \in \Omega$. Below, all variables are conditioned on $w$ and hence they are all deterministic. (For ease of notation we do not write $w$ in front of the variables).

$$
\begin{aligned}
& \Delta_{j}:=\sum_{\mathrm{t}=8 \alpha 2^{j-1}}^{8 \alpha 2^{j}} \frac{1}{\ell_{\sigma(\mathrm{t})}} \mathrm{f}^{\mathrm{s}_{\mathrm{t}-1}}\left(\mathrm{x}_{\sigma(\mathrm{t})}\right) \\
& =\sum_{\mathrm{t}=8 \alpha 2^{j}-1}^{8 \alpha 2^{j}} \frac{1}{\ell_{\sigma(\mathrm{t})}} \sum_{i \in[\mathrm{m}]: \mathrm{f}_{i}\left(s_{\mathrm{t}-1}\right)<1} f_{i}^{s_{\mathrm{t}-1}}\left(\mathrm{x}_{\sigma(\mathrm{t})}\right) \\
& \leqslant \sum_{t=8 \alpha 2^{j-1}}^{8 \alpha 2^{j}} \frac{1}{\ell_{\sigma(t)}} \sum_{i \in R_{j-1}} f_{i}^{s_{t-1}}\left(x_{\sigma(t)}\right) \\
& \leqslant \sum_{t \geqslant 1} \frac{1}{\ell_{\sigma(t)}} \sum_{i \in R_{j-1}} f_{i}^{s_{t-1}}\left(x_{\sigma(t)}\right) \\
& =\sum_{i \in R_{j-1}} \sum_{k=1}^{n} \frac{f_{i}\left(T_{k}\right)-f_{i}\left(T_{k-1}\right)}{1-f_{i}\left(T_{k-1}\right)}
\end{aligned}
$$


The first inequality uses the fact that any $i \notin R_{j-1}$ has $f_{i}$ already covered before time $8 \alpha 2^{j-1}$, and so it never contributes to $\Delta_{j}$. In the last expression, $\mathrm{T}_{\mathrm{k}}:=\left\{\mathrm{x}_{\pi(1)}, \ldots, \mathrm{x}_{\pi(\mathrm{k})}\right\} \subseteq \Delta$, the first $\mathrm{k}$ instantiations seen under $w$. The equality uses the fact that for each $\sum_{j=1}^{k-1} \ell_{\pi(j)}<t \leqslant \sum_{j=1}^{k} \ell_{\pi(j)}$ we have $s_{t-1}=T_{k-1}$ and $\sigma(t)=k$. Finally, by Claim 5.7 , the contribution of each function $f_{i} \in R_{j-1}$ is at most $\alpha:=1+\ln \frac{1}{\epsilon}$. Thus we obtain $\Delta_{j}(w) \leqslant \alpha\left|R_{j-1}(w)\right|$, and taking expectations,

$$
\Delta_{j} \leqslant \alpha \mathbb{E}\left[\left|R_{j-1}\right|\right]
$$

LOWER BOUND FOR $\Delta_{j}$. Consider any $8 \alpha 2^{j-1} \leqslant t \leqslant 8 \alpha 2^{j}$. We lower bound $G_{t}$. Condition on $s_{t-1}$; this determines $\sigma(t)$ (but not $x_{\sigma(t)}$ ). Note that $\sum_{i=1}^{n} \ell_{i} \cdot \operatorname{Pr}\left[X_{i} \in E_{j}^{*} \mid s_{t-1}\right] \leqslant 2^{j}$ by definition of $E_{j}^{*}$ being the elements that are completely scheduled by time $2^{j}$ in OPT. Hence we have

$$
\sum_{X_{i} \in \mathcal{A}} \frac{\ell_{i}}{2^{j}} \cdot \operatorname{Pr}\left[X_{i} \in E_{j}^{*} \mid s_{t-1}\right] \leqslant 1 .
$$

By applying Proposition 5.16 with the convex multipliers (over i) given above,

$$
\begin{aligned}
& \frac{1}{\ell_{\sigma(t)}} F^{s_{t-1}}\left(X_{\sigma(t)}\right) \\
& \geqslant \sum_{X_{i} \in \mathcal{A}} \frac{\ell_{i}}{2^{j}} \operatorname{Pr}\left[X_{i} \in E_{j}^{*} \mid s_{t-1}\right] \cdot \frac{1}{\ell_{i}} F^{s_{t-1}}\left(X_{i}\right) \\
& =\frac{1}{2^{j}} \sum_{X_{i} \in \mathcal{A}} \operatorname{Pr}\left[X_{i} \in E_{j}^{*} \mid s_{t-1}\right] \sum_{x_{i} \in \Delta} \operatorname{Pr}\left[X_{i}=x_{i} \mid s_{t-1}\right] \cdot f^{s_{t-1}}\left(x_{i}\right) \\
& =\frac{1}{2^{j}} \sum_{X_{i} \in \mathcal{A}} \sum_{x_{i} \in \Delta} \operatorname{Pr}\left[X_{i} \in E_{j}^{*} \wedge X_{i}=x_{i} \mid s_{t-1}\right] \cdot f^{s_{t-1}}\left(x_{i}\right) \\
& =\frac{1}{2^{j}} \sum_{w \in \Omega\left(s_{t-1}\right)} \operatorname{Pr}\left[w \mid s_{t-1}\right] \sum_{X_{i} \in E_{j}^{*}(w)} f^{s_{t-1}\left(X_{i}(w)\right)}
\end{aligned}
$$

The first equality is by definition of $F^{s_{t-1}}(\cdot)$ from (24). The second equality holds since the optimal policy must decide whether to schedule $X_{i}$ (by 
time $\left.2^{j}\right)$ without knowing the realization of $X_{i}$. Now for each $w \in \Omega\left(s_{t-1}\right)$, due to submodularity of the function $f^{s_{t-1}}(\cdot)$, we get

$$
\begin{aligned}
\sum_{X_{i} \in E_{j}^{*}(w)} f^{s_{t-1}}\left(X_{i}(w)\right) & \geqslant f^{s_{t-1}}\left(E_{j}^{*}(w)\right) \\
& =\sum_{i \in[m], f_{i}\left(s_{t-1}\right)<1} \frac{f_{i}\left(E_{j}^{*}(w)\right)-f_{i}\left(s_{t-1}\right)}{1-f_{i}\left(s_{t-1}\right)} \\
& \geqslant\left|C_{j}^{*}(w)\right|-|C(t, w)|
\end{aligned}
$$

Recall that $E_{j}^{*}(w)$ denotes the set of elements scheduled by time $2^{j}$ in OPT (conditional on $w$ ), as well as the realizations of these elements. The equality comes from the definition of $\mathrm{f}^{s_{t-1}}$. The last inequality holds because $C(t, w)=\left\{i \in[m]: f_{i}\left(s_{t-1}\right)=1\right\}$ and set $E_{j}^{*}(w)$ covers functions $\mathrm{C}_{\mathrm{j}}^{*}(\boldsymbol{w})$. Combining (28) and (29) gives:

$$
\frac{1}{\ell_{\sigma(t)}} F^{s_{t-1}}\left(X_{\sigma(t)}\right) \geqslant \frac{\left(\mathbb{E}\left[\left|C_{j}^{*}\right| \mid s_{t-1}\right]-\mathbb{E}\left[|C(t)| \mid s_{t-1}\right]\right)}{2^{j}} .
$$

By deconditioning the above inequality (taking expectation over $s_{t-1}$ ) and using (25), we derive:

$$
G_{t} \geqslant \frac{1}{2^{j}} \cdot\left(\mathbb{E}\left[\left|C_{j}^{*}\right|\right]-\mathbb{E}[|C(t)|]\right) \geqslant \frac{1}{2^{j}} \cdot\left(\mathbb{E}\left[\left|C_{j}^{*}\right|\right]-\mathbb{E}\left[\left|C_{j}\right|\right]\right),
$$

where the last inequality uses $\mathbb{E}[C(t)]$ is non-decreasing and $t \leqslant 8 \alpha 2^{j}$.

Now summing over all $t \in\left[8 \alpha 2^{j-1}, 8 \alpha 2^{j}\right)$ yields:

$$
\begin{aligned}
\Delta_{j} & =\sum_{t=8 \alpha 2^{j}-1}^{8 \alpha 2^{j}} G_{t} \geqslant 4 \alpha\left(\mathbb{E}\left[\left|C_{j}^{*}\right|\right]-\mathbb{E}\left[\left|C_{j}\right|\right]\right) \\
& =4 \alpha\left(\mathbb{E}\left[\left|R_{j}\right|\right]-\mathbb{E}\left[\left|R_{j}^{*}\right|\right]\right)
\end{aligned}
$$

Combining (31) and (27), we obtain:

$$
4 \alpha\left(\mathbb{E}\left[\left|R_{j}\right|\right]-\mathbb{E}\left[\left|R_{j}^{*}\right|\right]\right) \leqslant \alpha \mathbb{E}\left[\left|R_{j-1}\right|\right]
$$

which simplifies to the desired inequality in Lemma 5.15.

Theorem 5.14 now follows almost directly from Lemma 5.15, similar to the proof of Theorem 5.4 for Submodular Ranking in the first section of this chapter. 


\subsection{CONCLUSIONS}

In this chapter we claimed an significantly easier proof of the algorithm of Azar and Gamzu for Submodular Ranking [5]. This claim is supported by the ease we generalized the proof to obtain good results for Minimum Latency Submodular Cover and Weighted Stochastic Submodular Cover. In fact, our results for Weighted Stochastic Submodular Cover are tight, generalize and unify previous results. Further, our result for Minimum Latency Submodular Cover is almost tight, and any significant improvement would improve the approximation ratio for Group Steiner Tree: a long open-standing question.

As remarked before by Chekuri and Pál, their result gives a quasi-polynomial time $\mathrm{O}\left(\log ^{2}|\mathrm{~V}|\right)$-approximation algorithm. This implies, that unless NP has quasi-polynomial time algorithms, there is a polynomial time algorithm for Group Steiner Tree that gives a $\mathrm{O}\left(\log ^{2}|\mathrm{~V}|\right)$-approximation. 


\section{LATENCY COVERING STEINER TREE}

This chapter is based on part of the following paper:

S. Im, V. Nagarajan and R. van der Zwaan, "Minimum Latency Submodular Cover". Accepted at International Colloquium on Automata, Languages and Programming (ICALP) 2012.[63].

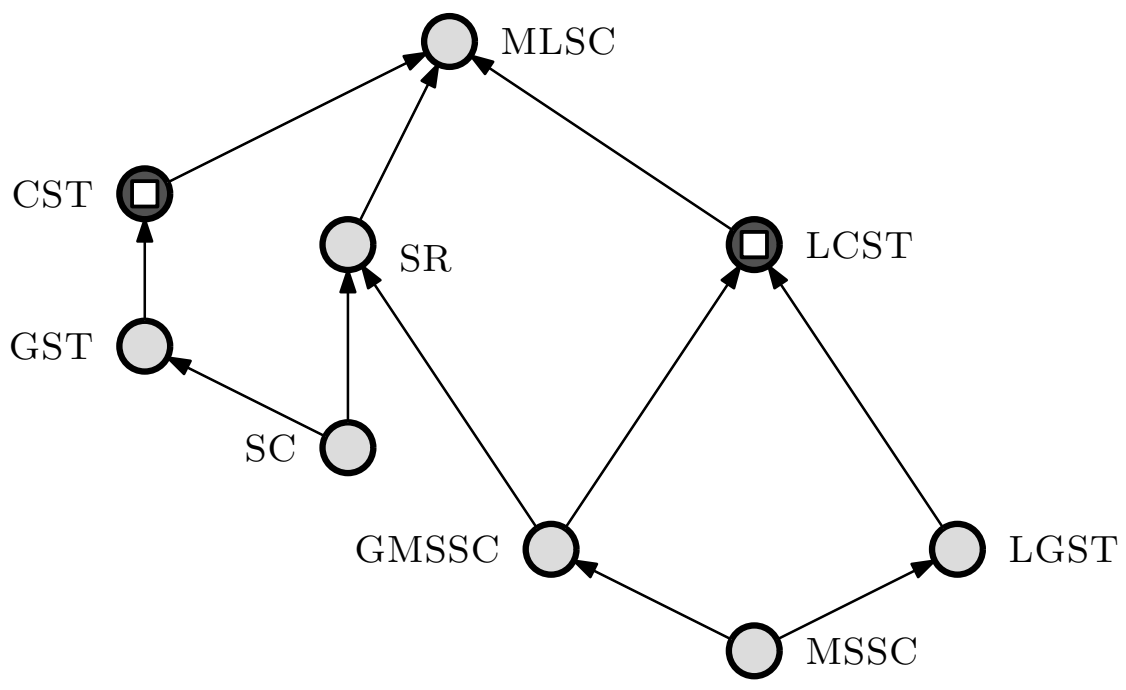




\subsection{INTRODUCTION}

The Latency Covering Steiner Tree problem (LCST) is a natural special case of Minimum Latency Submodular Cover (MLSC), where each function $f_{i}$ is associated with a group $g_{i} \subseteq V$ and requirement $k_{i} \leqslant\left|g_{i}\right|$ such that $f_{i}(S)=\min \left\{\frac{\left|g_{i} \cap S\right|}{k_{i}}, 1\right\}$. Note that in this case, $\epsilon=1 / \max _{i=1}^{m} k_{i}$. The uniform metric special case of LCST reduces to the Generalized Min-sum Set Cover problem $[6,8]$. When $\max _{i=1}^{m} k_{i}=1$ in LCST, we obtain the Latency Group Steiner Tree problem, studied in Gupta et al. [53] (see also [84]) and Chakrabarty-Swamy [23].

\subsubsection{Our results}

Our main result of this chapter is for the Latency Covering Steiner Tree (LCST) problem. Note that our result for the more general MLSC problem in Chapter 5 implies directly for LCST (i) polynomial time $\mathrm{O}\left(\log \mathrm{k}_{\max }\right.$. $\left.\log ^{2+\delta}|\mathrm{V}|\right)$-approximation and (ii) quasi-polynomial time $\mathrm{O}\left(\log \mathrm{k}_{\max } \cdot \log |\mathrm{V}|\right)$ approximation. Here $k_{\max }$ denotes the maximum requirement of any group in LCST.

Theorem 6.1. There is an $\mathrm{O}\left(\log ^{2}|\mathrm{~V}|\right)$-approximation algorithm for Latency Covering Steiner Tree.

Theorem 6.I improves on these bounds by providing a double logarithmic approximation in polynomial time. As shown in [84], any improvement over Theorem 6.I even in the $k_{\max }=1$ special case (i.e. Latency Group Steiner Tree ) would yield an improved approximation ratio for Group Steiner Tree, which is a long-standing open question.

Recall that LCST on a uniform metric is the Generalized Min Sum Set Cover problem, for which a constant factor approximation is known due to Bansal et al. [8]; the previous greedy-type algorithm [6] had a logarithmic ratio. A natural attempt at solving LCST is to extend the algorithm in [8] to general metrics. However, this is not straightforward since the [8] algorithm is LP-based, and there is no good LP relaxation known for the underlying Covering Steiner Tree problem. Although the $\mathrm{O}\left(\log N \log ^{2}|\mathrm{~V}|\right)$ approximation algorithm for Covering Steiner Tree [54, 76] uses an LP relaxation, that LP is known to have a large integrality gap [76]. The algorithms [54, 76] get around this integrality gap by iteratively (and adaptively) solving a sequence of such LPs; thus the final solution cost cannot 
be compared against a fixed LP relaxation. In context of the LCST problem it is unclear how to use this approach to get an $\mathrm{O}\left(\log ^{2}|\mathrm{~V}|\right)$-approximation. The adaptive residual coverage algorithm for MLSC is somewhat similar to adaptively solving LPs for covering Steiner tree; but that approach gives us only a weaker bound for LCST. We overcome this issue by proposing a new LP relaxation for Covering Steiner Tree, that has a small (poly-logarithmic) integrality gap. The new LP makes use of Knapsack Cover (KC) type inequalities [22]. We note that KC-inequalities (for set multicover) were also used in [8]. Still, it is non-trivial to formulate these stronger inequalities for Covering Steiner Tree in a way that they are useful in rounding. In particular, the separation oracle for the resulting $\mathrm{KC}$-inequalities is more complicated than for Set Multicover (which was the knapsack problem). Once we have the stronger LP relaxation for Covering Steiner Tree, we can use it in an LPbased algorithm for LCST using fairly standard techniques. These results are presented in Section 6.2 and Section 6.3, respectively.

\subsection{NEW LP RELAXATION FOR COVERING STEINER TREE}

We revisit the covering Steiner tree problem (CST) [54, 76]. Recall that its input consists of a metric $(\mathrm{V}, \mathrm{d})$ with root $\mathrm{r}$ and a collection of groups $\mathcal{G} \subseteq 2^{V}$ where each group $g \in \mathcal{G}$ is associated with a requirement $k_{g}$. The goal is to find a minimum cost $r$-rooted tree $\tau$ that includes $r$ and covers all groups. A group $g$ is covered if $\tau$ contains at least $k_{g}$ nodes from $g$.

Konjeved et al. [76] gave an $\mathrm{O}\left(\left(\log \mathrm{m}+\log \mathrm{k}_{\max }\right) \cdot \log g_{\max } \cdot \log n\right)$-approximation where $m$ is the number of groups, $k_{\max }:=\max _{g \in \mathcal{G}} k_{g}$ is the maximum requirement and $g_{\max }=\max _{g \in \mathcal{G}}|g|$ is the maximum group size. Later, Gupta and Srinivasan [54] gave an improved $\mathrm{O}\left(\log \mathrm{m} \cdot \log g_{\max }\right.$. $\log n)$-approximation that has no dependence on $k_{\max }$. In certain settings of parameters, the result [54] yields an improvement of $\Theta\left(\frac{\log n}{\log \log n}\right)$ over [76]. Both these previous works use the same LP that has a large integrality gap of $\Omega\left(k_{\max }\right)$ [76]. To circumvent this, they solve a sequence of LPs adaptively: In each step, the requirement of each group is partially satisfied (by a constant fraction), and the subsequent LP is defined over the residual requirement and the unvisited vertices. 

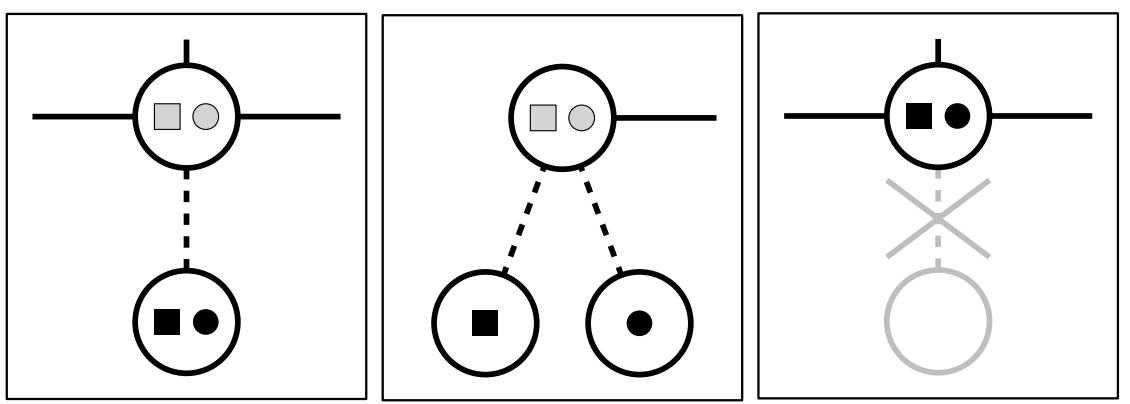

Figure 9: Simplifying assumptions 2, 3 and 4. The light square means that the vertex is removed from the group "square" and the dark square indicates the vertex is added to the group "square".

We introduce stronger constraints, that yield an LP for CST with integrality gap $\mathrm{O}\left(\log \mathrm{m} \cdot \log g_{\max } \cdot \log n\right)$. This new LP is an important ingredient in our algorithm for LCST (next section), and might be useful in other contexts as well.

\subsubsection{Simplifying assumptions}

Following $[76,54]$, without loss of generality, we proceed our analysis under the following simplifying assumptions.

1. The given graph is a (weighted) tree $T=(V, E)$ with root $r$ and weight $w_{e}$ on each edge $e \in E$.

2. Every vertex in a group is a leaf, i.e. has degree 1.

3. The groups in $\mathcal{G}$ are disjoint.

4. Every vertex of degree 1 is in a group.

For assumption (1), the tree embedding in [39], a $\rho$-approximation on tree instances immediately implies a randomized $\mathrm{O}(\rho \log |\mathrm{V}|)$-approximation on general instances. Hence we focus on giving an $\mathrm{O}\left(\log \mathrm{m} \cdot \log g_{\max }\right)$ approximation on tree instances. The simplifying assumptions (2) to (4) are depicted in Figure 9. Assumption (2) follows from the following operation. Suppose there is a vertex $v$ that is in at least one group but with degree at least two. Create an extra vertex $v^{\prime}$ and insert the edge $\left\{v, v^{\prime}\right\}$ 
with weight 0 and finally replace $v$ by $v^{\prime}$ in all groups $v$ was present. Assumption (3) we have a degree 1 vertex $v$ that is in groups $g_{1}, \ldots, g_{k}$. Add a vertex $v_{i}$ and edge $\left\{v, v_{i}\right\}$ with weight 0 and add $v_{i}$ to group $g_{i}$ for $1 \leqslant i \leqslant k$, finally remove $v$ from all groups. Assumption (3) is due to the fact that if a vertex of degree 1 is not in any group, the incident edge is not needed in the optimal solution.

\subsubsection{Linear Program}

We let L denote the set of leaves in V. Because of the above simplifying assumptions, we can label each vertex $v$ in a group with a unique leaf-edge incident on it, and vice versa. We abuse notation by allowing $j \in L$ to denote both the leaf-vertex and its unique incident edge. For any subset of leaves $L^{\prime} \subseteq L$, let cut $\left(r, L^{\prime}\right)$ denote the family of all sets of edges such that their removal separates the root $r$ from all vertices in $L^{\prime}$. For notational convenience, we assume that there is a pseudo-edge incident on $r$ that is an ancestor of all edges in E. We call this edge super-root and assume that it is alway included in our solution and has zero cost. Hence for any edge $j \in E$, its parent edge, denoted as $p e(j)$, is well-defined.

We formulate the following linear programming relaxation for CST on tree instances.

$$
\begin{array}{lll}
\min & \sum_{e \in \mathrm{E}} w_{e} x_{e} & \\
\text { s.t. } & x_{p e(e)} \geqslant x_{e} & \\
& \left(k_{g}-|A|\right) \sum_{j \in B \backslash L} x_{j}+\sum_{j \in B \cap(L \backslash A)} x_{j} \geqslant k_{g}-|A| & \\
& \forall g \in \mathcal{G}, \forall A \subseteq g, \forall B \in \operatorname{cut}(r, g \backslash A) & \\
& x_{e} \in[0,1] & \forall e \in E
\end{array}
$$

We first argue that this is a valid relaxation. Consider any instance of CST on trees and a fixed feasible solution (tree) $\tau^{*}$, which gives a natural integral solution: $x_{e}=1$ if and only if $e \in \tau^{*}$. We focus on constraints (33), since the other constraints are obviously satisfied. Consider any $g \in \mathcal{G}$, $A \subseteq g$ and $B \in \operatorname{cut}(r, g \backslash A)$. Let $\tau^{*}(E \backslash A)$ denote the subtree induced by the edges in both $\tau^{*}$ and $(E \backslash A)$, i.e. $\tau^{*} \cap(E \backslash A)$. Note that $\tau^{*}(E \backslash A)$ is connected, since $A$ consists only of leaf edges. Since $\tau^{*}$ has at least $k_{g}$ edges in $g$ (it is a feasible CST solution), $\left|\tau^{*}(E \backslash A) \cap(g \backslash A)\right| \geqslant k_{g}-|A|$. 
- Suppose that there exists $\bar{j} \in \tau^{*}(E \backslash A) \cap B$ such that $\bar{j} \notin$ L. Then since $\bar{j} \in B \backslash L$, it follows that $\left(k_{g}-|A|\right) \sum_{j \in B \backslash L} x_{j} \geqslant k_{g}-|A|$, hence the constraint is satisfied.

- The remaining case has $\tau^{*}(E \backslash A) \cap B \subseteq$ L. In words, B cuts $g \backslash$ $A$ from $r$ using only leaf edges; so $B \supseteq \tau^{*}(E \backslash A) \cap(g \backslash A)$. Thus $\sum_{j \in B \cap(L \backslash A)} x_{j} \geqslant\left|\tau^{*}(E \backslash A) \bigcap(g \backslash A)\right| \geqslant k_{g}-|A|$.

In either of the above cases, constraint (33) is satisfied.

\subsubsection{Solving the LP}

Since LP CST has exponentially many constraints, in order to solve it in polynomial time, we need a separation oracle. Again we focus on constraints (33), since other constraints are only polynomially many. We observe such an oracle is reduced to finding a polynomial time algorithm for the following problem.

Problem MinCutWithExceptions: In this problem, we are given as input a tree $T$ rooted at $r$ with leaves $L$ and cost $\ell(e)$ on each edge $e$. Further, an integer $\mathrm{D} \geqslant 0$ is given. The goal is to find a minimum cost cut that separates $r$ from any $D$ leaves.

We first explain how the desired separation oracle is reduced to solving the above problem. Consider the constraints (33) for a fixed $g \in \mathcal{G}$ and all $A \subseteq g$ with $|A|=\eta$ (finally we iterate over all $g \in \mathcal{G}$ and $0 \leqslant \eta \leqslant n$ ). Then $k_{g}-|A|$ (the right-hand-side of the constraints) is also fixed. Given $x_{j}$ values, we would like to find $A \subseteq g$ with $|A|=\eta$ and $B \in \operatorname{cut}(r, g \backslash A)$ that minimizes the left-hand-side, and test if this is smaller than $k_{g}-\eta$. Formally, we can recast this into MinCutWithExceptions as follows: Remove all edges from $E$ that are not on any path from the root $r$ to a vertex in $g$, and let $T^{\prime}$ be the resulting tree and this is the input tree to the problem. Note that leaves of $T^{\prime}$ are precisely $g$. For all leaf-edges $j \in g$, let $\ell(j):=x_{j}$; and for all non-leaf $j \in T^{\prime} \backslash g, \ell(j):=\left(k_{g}-\eta\right) \cdot x_{j}$. Also set bound $D:=|g|-\eta$.

We next show that MinCutWithExceptions can be solved via a dynamic programming.

Lemma 6.2. The problem MinCutWithExceptions can be solved in polynomial time.

Proof. To formally describe our dynamic program, we make some simplifying assumptions. By introducing dummy edges of infinite cost, we assume 


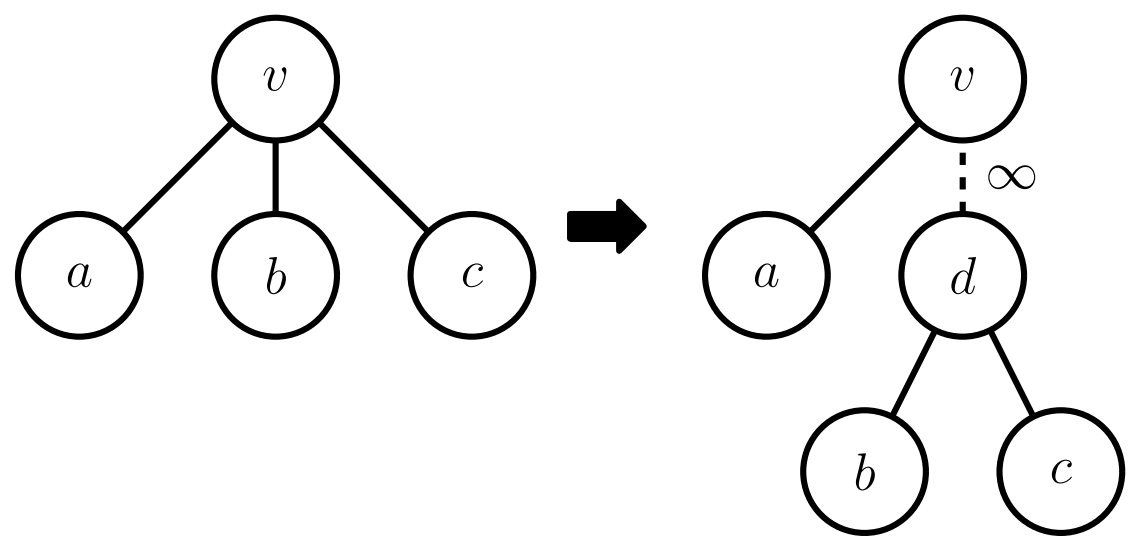

Figure 10: Illustration of how the tree can be ensured to be binary by inserting a dummy node $d$.

without loss of generality that the tree $T$ is binary and the root $r$ is incident to exactly one edge $e_{r}$ (See Figure 10). Hence every non-leaf edge $e$ has exactly two child-edges $e_{1}$ and $e_{2}$. For any edge $e \in T$ let $T_{e}$ denote the subtree of $T$ rooted at $e$, i.e. $T_{e}$ contains edge $e$ and all its descendants.

We define a recurrence for $\mathrm{C}[e, \mathrm{k}]$ which denotes the minimum cost cut that separates the root of $T_{e}$ from exactly $k$ leaves in $T_{e}$. Note that $C\left[e_{r}, D\right]$ gives the optimal value in polynomial time.

For any leaf-edge $f$ set:

$$
C[f, k]= \begin{cases}0 & \text { if } k=0, \\ \ell(f) & \text { if } k=1, \text { and } \\ \infty & \text { otherwise. }\end{cases}
$$

For any non-leaf edge $e$ with children $e_{1}$ and $e_{2}$, set:

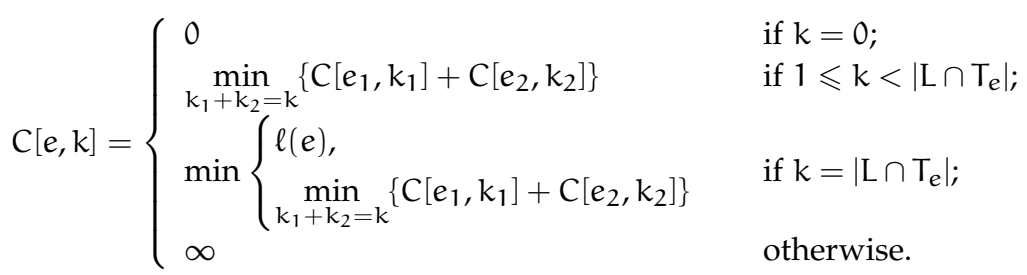

It can be checked directly that this recurrence computes the desired values. 


\subsubsection{Algorithm}

Our algorithm uses threshold rounding and then applies the elegant rounding scheme from [76] (to a suitably modified fractional solution). In this respect it is somewhat similar to the algorithm in [54]; however that approach solves linear programs and rounds them iteratively. Whereas our algorithm uses a single LP. We describe the Konjevod-Ravi-Srinivasan (KRS) rounding scheme first and then our main algorithm.

The rounding scheme in [76]

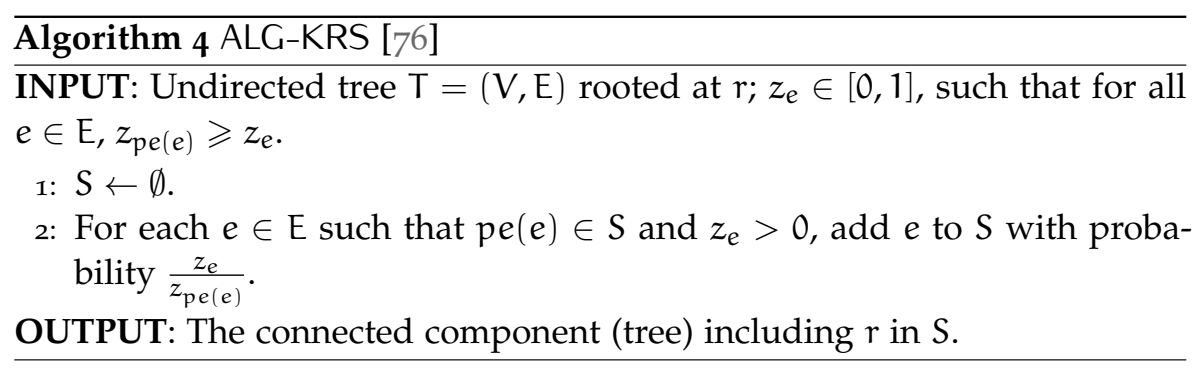

We have the following proposition from Konjevod, Ravi and Srinivasan.

Proposition 6.3 ([76]). Each edge e is included in the final solution of ALG-KRS with probability $z_{e}$.

Proof. We prove this by induction. Assume that for the induction hypothesis that an edge $e$ its parent edge pe(e) is included in the final solution with probability $z_{\text {pe(e) }}$. Then, by the algorithm $e$ is included with probability $z_{\text {pe }(e)} \cdot \frac{z_{e}}{z_{p e(e)}}=z_{e}$. The super-root edge, the only edge without a parent edge, is included with probability 1, so the base case for the induction holds.

Definition 6.4 (KRS properties). For all $\mathrm{g} \in \mathcal{G}$, consider any $\mathrm{R}(\mathrm{g}) \subseteq \mathrm{g}$ and any $0 \leqslant r_{g} \leqslant|R(g)|$. We say that $\left(z, R(g), r_{g}\right)$ satisfies the KRS properties if it satisfies the following properties.

$$
\begin{aligned}
z_{p e(e)} \geqslant z_{e} & \forall e \in E \\
\sum_{j \in T(e) \cap R(g)} z_{j} \leqslant r_{g} z_{e} & \forall e \in E
\end{aligned}
$$

where $\mathrm{T}(\mathrm{e})$ is the subtree below (and including) edge e. 
The first property (34) is the same as the constraints (32). The second property (35) is a Lipschitz-type condition that says conditional on any edge $e$ being chosen, its subtree $T(e)$ can contribute at most $r_{g}$ to the requirement of $R(g)$. We remark that this property (35) was enforced explicitly in $[76,54]$ as constraints (for specific $R(g)$ and $r_{g}$ ) in their sequence of LPs. On the other hand, as we show later, our LP constraint (33) directly gives this property for all relevant $R(g)$ and $r_{g}$ values.

Lemma $6.5([76])$. Suppose that $\left(z, R(g), r_{g}\right)$ satisfies the KRS properties. Let $\mathrm{L}_{\mathrm{krs}}$ denote the set of leaves that are covered by $A L G-K R S$ with the input $z_{e}, e \in \mathrm{E}$. Consider any constant $\delta \in[0,1]$. Then for any $\mathrm{g} \in \mathcal{G}$, we have

$$
\operatorname{Pr}\left[\left|L_{k r s} \cap R(g)\right| \leqslant(1-\delta) \mu_{g}\right] \leqslant \exp \left(-\frac{\delta^{2} \cdot \mu_{g}}{2+r_{g}(1+\ln |R(g)|)}\right)
$$

where $\mu_{g}:=\mathbb{E}\left[\left|\mathrm{L}_{k r s} \cap R(g)\right|\right]=\sum_{j \in R(g)} z_{j}$.

Proof. We only give a quick sketch of the proof, since this is implicit in [76]. For any $j, j^{\prime} \in R(g)$, we say that $j \sim j^{\prime}$ if and only if $(1) j \neq j^{\prime}$ and (2) the least common ancestor $l c a\left(j, j^{\prime}\right)$ of $j$ and $j^{\prime}$ is not $r$. Define

$$
\Delta_{g}:=\sum_{j, j^{\prime} \in R(g): j j^{\prime}, z_{l c a\left(j, j^{\prime}\right)}>0} \frac{z_{j} \cdot z_{j^{\prime}}}{z_{l c a\left(j, j^{\prime}\right)}}
$$

In Theorem 3.2 in [76], Konjevod et al. showed using the KRS properties that

$$
\Delta_{g} \leqslant \mu_{g}\left(r_{g}-1+r_{g} \ln |R(g)|\right)
$$

We note that the proof of Theorem 3.2 implies this, although it is stated only for $\mu_{g}=r_{g}$. Further, they used this bound in Jansen's inequality to obtain for any $\delta \in[0,1]$,

$$
\operatorname{Pr}\left[\left|\mathrm{L}_{k r s} \cap R(g)\right| \leqslant(1-\delta) \mu_{g}\right] \leqslant \exp \left(-\frac{\delta^{2} \mu_{g}}{2+\Delta_{g} / \mu_{g}}\right)
$$

Above $\mu_{g}=\mathbb{E}\left[\left|L_{k r s} \cap R(g)\right|\right]=\sum_{j \in R(g)} \operatorname{Pr}\left[j \in L_{k r s}\right]=\sum_{j \in R(g)} z_{j}$, by Proposition 6.3. Combining the above two inequalities yields the lemma.

\section{Main algorithm}

The algorithm first preprocesses an optimal solution $x^{*}$ to LP CST to obtain another solution $x^{\prime}$ that is used in the KRS-rounding. Let $E^{*}:=\left\{e \in E \mid x_{e}^{*} \geqslant\right.$ 
$\left.\frac{1}{2}\right\}$; all edges in $E^{*}$ are included in our solution. Observe that $E^{*}$ forms a tree rooted at $r$ due to the constraints (33). We let $\mathrm{T}^{\prime}=\mathrm{T} / \mathrm{E}^{*}$ denote the tree obtained by contracting edges $E^{*}$ in the original tree $T$; and $E^{\prime}=E \backslash E^{*}$ the edges of $T^{\prime}$. For any group $g \in \mathcal{G}$, define $R(g):=g \backslash E^{*}$ and $r_{g}:=$ $k_{g}-\left|g \cap E^{*}\right|$. Since $E^{*}$ already covers the vertices in $g \cap E^{*}$, we are henceforth concerned with getting coverage of at least $r_{g}$ from vertices $R(g)$. Our algorithm ALG-CST is formally described below. Note that for any $e \in E^{\prime}$, $z_{e}^{\prime}=2 x_{e}^{\prime} \leqslant 1$ and $z_{p e(e)}^{\prime} \geqslant z_{e}^{\prime}$, hence $z^{\prime}$ is a valid input to ALG-KRS with respect to $T^{\prime}$. The final output of the algorithm is the union of all sampled trees $\tau$ plus the edges in $E^{*}$ that are initially shrunk.

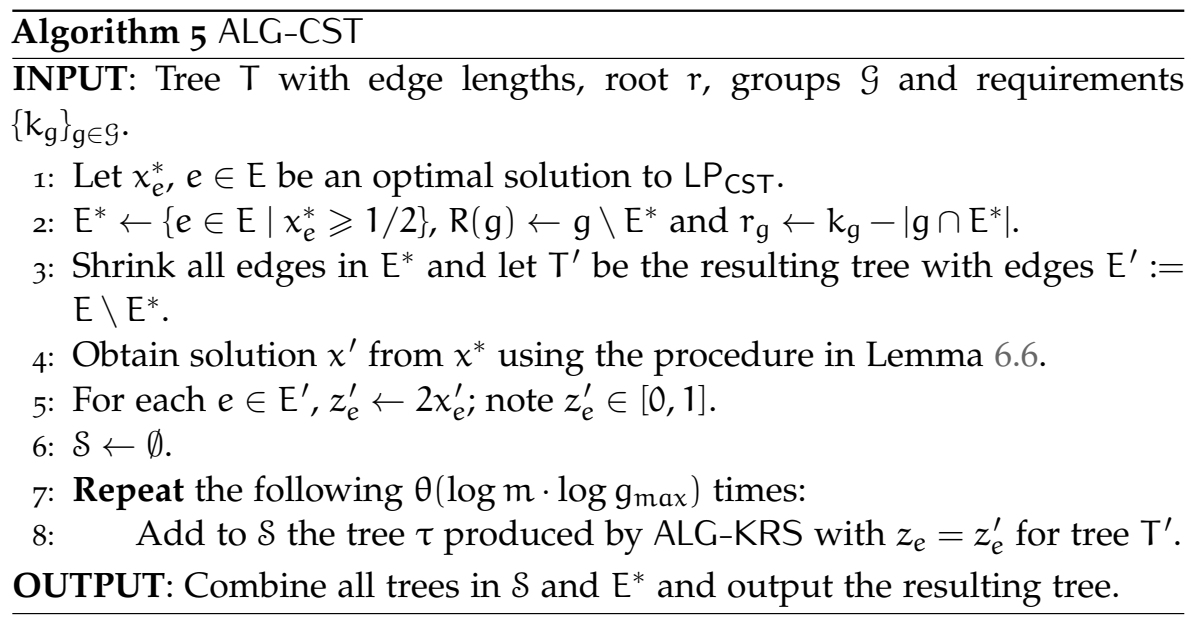

Lemma 6.6. There exist $x_{e}^{\prime} \in\left[0, x_{e}^{*}\right]$ for all $e \in E \backslash E^{*}$ such that for all $g \in \mathcal{G}$, $\left(x^{\prime}, R(g), r_{g}\right)$ satisfies the KRS properties in tree $T^{\prime}$ and $\sum_{j \in R(g)} x_{j}^{\prime} \geqslant r_{g}$. Furthermore, such $\mathrm{x}^{\prime}$ can be computed in polynomial time.

Proof. Consider constraints (33) of LPCST. Fix a group g $\in \mathcal{G}$ and let $A:=$ $g \cap E^{*}$. Consider tree $T^{\prime}$ as a flow network with each leaf edge $f$ having capacity $\chi_{f}^{*}$ and each non-leaf edge $e$ having capacity $r_{g} \cdot x_{e}^{*}$. The root $r$ is the source and leaves $R(g)=g \backslash A$ are the sinks. Then constraints (33) imply that the min cut separating $r$ from $R(g)$ has value at least $r_{g}$ : note that although these constraints are for the original tree $T$, they imply similar constraints for $T^{\prime}$ since $T^{\prime}$ is obtained from $T$ by edge-contraction. ${ }^{1}$ Hence

I In particular every cut $B^{\prime}$ separating $r$ from $g \backslash A$ in $T^{\prime}$ is also a cut separating $r$ from $g \backslash A$ in $\mathrm{T}$. 
there must exist a max-flow of volume at least $r_{g}$ from $r$ to $R(g)$ in the above network. Let $x_{f}^{\prime}$ denote the volume of this flow into each leaf edge $f \in R(g)$; clearly we have that $x_{f}^{\prime} \leqslant x_{f}^{*}$ (due to capacity on leaves) and:

$$
\sum_{j \in R(g)} x_{j}^{\prime} \geqslant r_{g} .
$$

Moreover, by the capacities on non-leaves,

$$
\sum_{j \in T(e) \cap R(g)} x_{j}^{\prime} \leqslant r_{g} x_{e}^{*} \quad \forall e \in E
$$

We can use the above procedure on each group $g \in \mathcal{G}$ separately to compute $x_{f}^{\prime}$ for all leaf edges $f$; this is well-defined since groups are disjoint. For each non-leaf edge $e \in E^{\prime}$ set $x_{e}^{\prime}:=x_{e}^{*}$. Thus we have $0 \leqslant x_{e}^{\prime} \leqslant x_{e}^{*}$ for all $e \in E^{\prime}$. Observe that this computation can easily be done in poly-time.

Now, (37) implies the second KRS property (35). Property (34) follows, since for each $e \in E^{\prime}$, we have $x_{p e(e)}^{\prime}=x_{p e(e)}^{*} \geqslant x_{e}^{*} \geqslant x_{e}^{\prime}$; the first inequality is due to constraint (32) of LPCST. Finally (36) implies the coverage property claimed in the lemma.

\subsubsection{Analysis}

Consider a random tree $\tau$ in Line (8) of ALG-CST. For a formal analysis, we assume that ALG-CST samples $\tau, 4 \log m\left(3+\ln g_{\text {max }}\right)$ times independently. For each group $g \in \mathcal{G}$, we focus on lower bounding the probability that $\tau$ satisfies the residual requirement $r_{g}$ for $R(g)$.

Lemma 6.7. For any group $\mathrm{g} \in \mathcal{G}$,

$$
\operatorname{Pr}\left[|\tau \cap R(g)|<r_{g}\right] \leqslant \exp \left(-\frac{1}{2\left(3+\ln g_{\max }\right)}\right) .
$$

Proof. Consider any group $g \in \mathcal{G}$. By Lemma 6.6, we know $\left(x^{\prime}, R(g), r_{g}\right)$ satisfies the KRS properties with respect to tree $T^{\prime}$. From the definition of $z_{e}^{\prime}=2 \cdot x_{e}^{\prime}$ and that $z_{e}^{\prime} \leqslant 1$ for all $e \in \mathrm{E}^{\prime}$, it immediately follows that $\left(z^{\prime}, R(g), r_{g}\right)$ also satisfies the KRS properties on $T^{\prime}$. Further, by Proposition 6.3 , it follows

$$
\mu_{g}:=\mathbb{E}[|\tau \cap R(g)|]=\sum_{j \in R(g)} z_{j}^{\prime}=2 \sum_{j \in R(g)} x_{j}^{\prime} \geqslant 2 r_{g},
$$


where we used the coverage property $\sum_{j \in R(g)} x_{j}^{\prime} \geqslant r_{g}$ given by Lemma 6.6. By applying Lemma 6.5 with $\delta=1 / 2$, we have

$$
\begin{aligned}
& \operatorname{Pr}\left[|\tau \cap R(g)|<r_{g}\right] \leqslant \exp \left(-\frac{r_{g}}{2\left(2+r_{g}(1+\ln |R(g)|)\right)}\right) \\
= & \exp \left(-\frac{1}{2(3+\ln |R(g)|}\right) \leqslant \exp \left(-\frac{1}{2\left(3+\ln g_{\max }\right)}\right)
\end{aligned}
$$

Above we used $r_{g} \geqslant 1$ and $|R(g)| \leqslant g_{\text {max }}$.

It follows that upon sampling the tree $\tau$ independently $4 \ln \mathrm{m} \cdot\left(3+\ln g_{\text {max }}\right)$ times, each group $g \in \mathcal{G}$ is covered with probability at least $1-\frac{1}{\mathrm{~m}^{2}}$. A simple union bound yields that all groups are covered by the output of ALG-CST with probability at least $1-1 / \mathrm{m}$.

Now we upper bound the expected cost of the final solution.

Lemma 6.8. With at least probability $1 / 2$, the final solution of ALG-CST does not cost more than $8 \log \mathrm{m}\left(3+\ln \mathrm{g}_{\max }\right)$ times the optimum.

Proof. From Proposition 6.3, we know that the expected cost of $\tau$ is

$$
\sum_{e \in E \backslash E^{*}} z_{e}^{\prime} \leqslant 2 \sum_{e \in E \backslash E^{*}} x_{e}^{\prime} \leqslant 2 \sum_{e \in E \backslash E^{*}} x_{e}^{*} .
$$

Hence, knowing that $x_{e}^{*} \geqslant 1 / 2$ for all $e \in E^{*}$, the expected cost of the final solution is at most

$$
4 \log m\left(3+\ln g_{\max }\right) \sum_{e \in E \backslash E^{*}} w_{e} x_{e}^{*}+\sum_{e \in E^{*}} w_{e} \leqslant 4 \log m\left(3+\ln g_{\max }\right) \text { OPT. }
$$

Markov inequality yields the lemma.

Hence we derive the following theorem.

Theorem 6.9 ([54]). There is a randomized $\mathrm{O}\left(\log \mathrm{m} \cdot \log \mathrm{g}_{\max }\right)$-approximation algorithm for CST on tree metrics. For general metrics, there is a randomized $\mathrm{O}\left(\log \mathrm{m} \cdot \log \mathrm{g}_{\max } \cdot \log |\mathrm{V}|\right)$-approximation algorithm.

\subsection{LATENCY COVERING STEINER TREE PROBLEM}

In this section, we give an improved algorithm for the Latency Covering Steiner Tree problem (LCST), which is an interesting special case of 
MLSC. Recall that the input to LCST consists of a symmetric metric $(V, d)$, root $r \in \mathrm{V}$ and a collection $\mathcal{G}$ of $\mathrm{m}$ groups with associated requirements $\left\{k_{g}\right\}_{g \in \mathcal{G}}$. The goal is find a path staring from $r$ that minimizes the total cover time of all groups. We say that group $g$ is covered at the earliest time $t$ when the path within distance $t$ visits at least $k_{g}$ vertices in $g$. We give an $\mathrm{O}\left(\log g_{\max } \cdot \log |\mathrm{V}|\right)$-approximation algorithm for this problem where $g_{\max }:=\max _{\mathrm{g} \in \mathcal{G}}|\mathrm{g}|$ is the maximum group size.

The stronger constraints (33), which we introduced in the previous section, play a crucial role in our algorithm and analysis for LCST as well. We make the same simplifying assumptions that we made in Section 6.2.I. In particular, in order to obtain an $\mathrm{O}\left(\log g_{\max } \cdot \log |\mathrm{V}|\right)$-approximation on general instances, it suffices to give an $\mathrm{O}\left(\log g_{\max }\right)$-approximation on tree instances. We follow most of the notation and explain any difference in notation if and when necessary.

\subsubsection{Linear Programming Relaxation}

We formulate the following linear relaxation for tree instances of LCST.

$$
\begin{aligned}
& \min \frac{1}{2} \cdot \sum_{\ell \geqslant 0} 2^{\ell} \sum_{g \in \mathcal{G}}\left(1-y_{g}^{\ell}\right) \\
& \text { s.t. } \quad x_{p e(e)}^{\ell} \geqslant x_{e}^{\ell} \quad \forall \ell \geqslant 0, e \in E \\
& \sum_{j \in E} w_{e} x_{e}^{\ell} \leqslant 2^{\ell} \quad \forall \ell \geqslant 0 \\
& \left(k_{g}-|A|\right) \sum_{j \in B \backslash L} x_{j}^{\ell}+\quad \sum_{j \in B \cap L \backslash A} x_{j}^{\ell} \geqslant\left(k_{g}-|A|\right) \cdot y_{g}^{\ell} \\
& \forall \ell \geqslant 0, g \in \mathcal{G}, A \subseteq g, \quad B \in \operatorname{cut}(r, g \backslash A) \\
& y_{g}^{\ell+1} \geqslant y_{g}^{\ell} \quad \forall \ell \geqslant 0, g \in \mathcal{G} \\
& x_{e}^{\ell} \in[0,1] \quad \forall \ell \geqslant 0, e \in E \\
& y_{g}^{\ell} \in[0,1] \quad \forall \ell \geqslant 0, g \in \mathcal{G}
\end{aligned}
$$

To see that this is a valid relaxation, let OPT denote the optimal path. For any $\ell \geqslant 0$ let OPT $\left(2^{\ell}\right)$ denote the prefix of length $2^{\ell}$ of OPT. We construct a feasible integral solution for OPTas follows. The variable $x_{e}^{\ell}$ indicates if edge $e$ is covered by OPT $\left(2^{\ell}\right)$. The indicator variable $y_{g}^{\ell}$ has value one if and only if group $\mathrm{g}$ is covered by $\mathrm{OPT}\left(2^{\ell}\right)$. Constraints (38) come from the 
fact that OPT $\left(2^{\ell}\right)$ is a path starting at $r$. Constraints (39) say that the edges in $\mathrm{OPT}\left(2^{\ell}\right)$ have a total weight of at most $2^{\ell}$, which is clearly true. Note that for each $\ell \geqslant 0$, we created a set of constraints (40) that are essentially equivalent to the constraints (33) of LP CST. Constraints (40) can be shown to be valid similarly as are the constraints (33). Constraints (4I) enforce the fact that if group $g$ is covered by $\mathrm{OPT}\left(2^{\ell}\right)$ and it must be covered by $\mathrm{OPT}\left(2^{\ell+1}\right)$ as well. Now consider the objective value: the total contribution of a group $g$ that is covered by OPTat time $\in\left(2^{k}, 2^{k+1}\right]$ is $\frac{1}{2} \cdot \sum_{\ell=0}^{k} 2^{\ell} \leqslant 2^{k}$. Thus the objective value of this integral solution is at most OPT.

It is easy to see that it suffices to consider $\ell$ of polynomial value: so the number of variables is polynomial. Note that constraints (40) are exponentially many. However, for each fixed $\ell$, we can use essentially the same separation oracle that we used for the constraints (33) of LPCST.

\subsubsection{Algorithm}

Let $(\bar{x}, \bar{y})$ denote a fixed optimal solution to LP LCST. Let $\bar{E}^{\ell}:=\left\{e \in E \mid \bar{\chi}_{e}^{\ell} \geqslant\right.$ $1 / 4\}$. Observe that $\bar{E}^{\ell}$ forms a tree rooted at $r$ due to the constraints (38). The edges in $\bar{E}^{\ell}$ are added to our solution with probability 1 by the $\ell$-th phase of our algorithm. Let $R^{\ell}(g):=g \backslash \bar{E}^{\ell}$ and $r_{g}^{\ell}=k_{g}-\left|g \cap \bar{E}^{\ell}\right|$. Our algorithm tries to satisfy the residual requirement $r_{g}^{\ell}$ of group $g$ by covering vertices in $R^{\ell}(g)$ when the group is substantially covered (say to extent $\frac{1}{2}$ ) by the optimal fractional solution $(\bar{x}, \bar{y})$.

For each $\ell \geqslant 0$ we preprocess $\bar{x}^{\ell}$ and obtain $\tilde{x}^{\ell}$ that have "good" properties.

Lemma 6.10. For any $\ell \geqslant 0$, we can find in polynomial time $\tilde{x}_{e}^{\ell} \in\left[0, \bar{x}_{e}^{\ell}\right], \forall e \in$ $\mathrm{E} \backslash \overline{\mathrm{E}}^{\ell}$ such that $\forall \mathrm{g} \in \mathrm{G}$ :

1. $\left(\tilde{x}, R^{\ell}(g), r_{g}^{\ell}\right)$ satisfies the KRS-properties in tree $\tilde{T}^{\ell}$.

2. $\sum_{j \in R^{\ell}(g)} \tilde{x}_{j}^{\ell} \geqslant r_{g}^{\ell} \cdot \bar{y}_{g}^{\ell}$ (coverage property).

Proof. The proof of this lemma is very similar to Lemma 6.6. For each $\ell$, we obtain the desired $\tilde{x}^{\ell}$ values from $\bar{x}^{\ell}$ by taking similar steps as we did to set $x^{\prime}$ from $x^{*}$ in the proof of Lemma 6.6.

We now describe our algorithm ALG-LCST as follows. We say that ALG-LCST is in phase $\ell$ based on the value of $\ell$ in the description. 


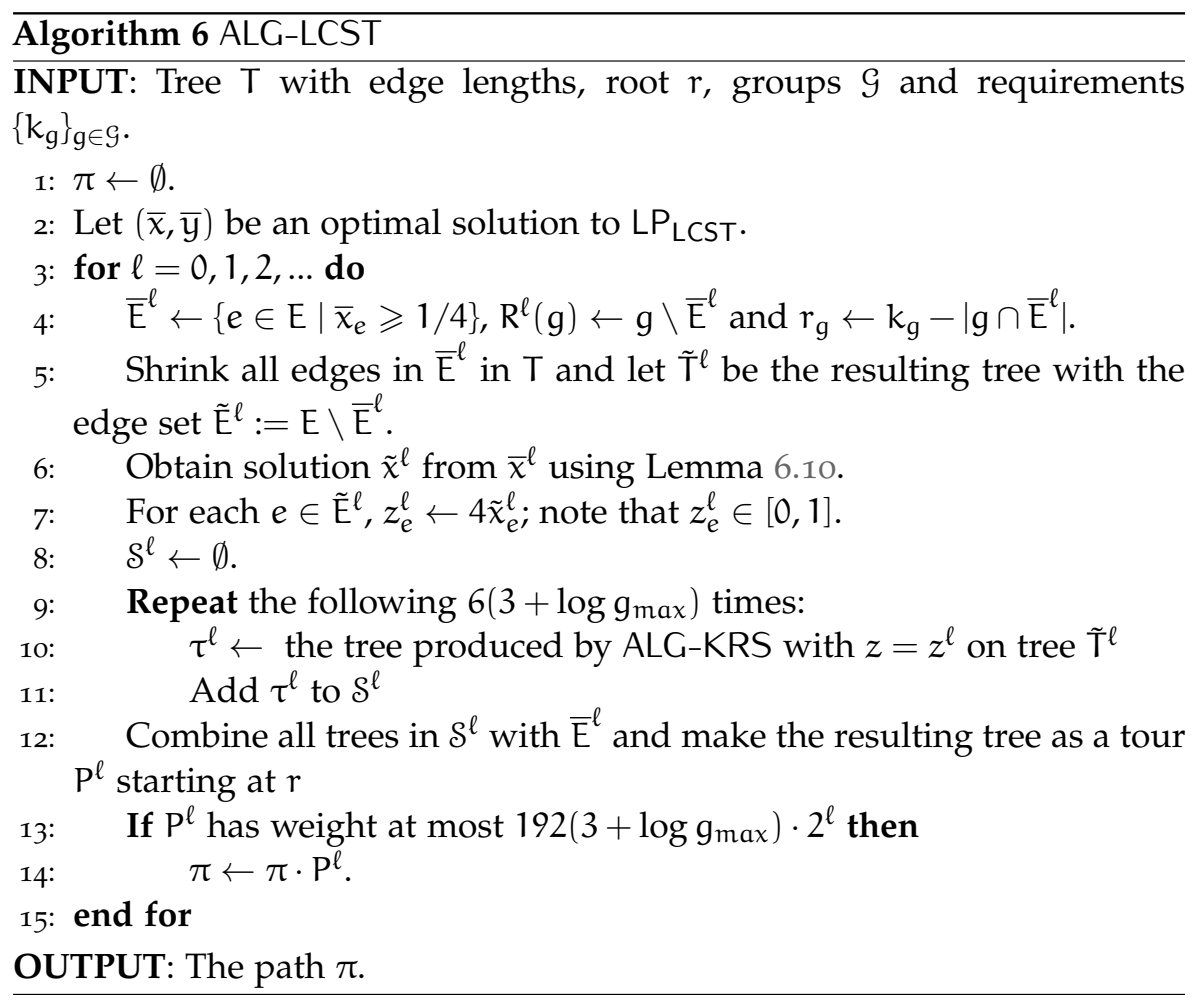

\subsubsection{Analysis}

For any group $g$, define $\ell(g)$ as the smallest $\ell \geqslant 0$ such that $\bar{y}_{g}^{\ell} \geqslant 1 / 2$. Then it follows that for any $\ell \geqslant \ell(g), \bar{y}_{g}^{\ell} \geqslant 1 / 2$ due to constraints (4I) of LPLCST. In words, the optimal fractional solution covers group $g$ by at least half within time $2^{\ell(g)}$. Consider any group $g \in \mathcal{G}, \ell \geqslant \ell(g)$ and a tree $\tau^{\ell}$ in Line (10) of ALG-LCST. Since all edges in $\overline{\mathrm{E}}^{\ell}$ are included in $\mathrm{P}^{\ell}$ with probability 1 , group $g$ is covered by $P^{\ell}$ if and only if at least $r_{g}^{\ell}$ vertices in the residual group $R^{\ell}(g)$ are covered by $\tau^{\ell}$. This motivates us to derive the following lemma.

Lemma 6.11. For any $\mathrm{g} \in \mathcal{G}$ and $\ell \geqslant \ell(\mathrm{g})$,

$$
\operatorname{Pr}\left[\left|\tau^{\ell} \cap R(g)\right|<r_{g}\right] \leqslant \exp \left(-\frac{1}{2\left(3+\ln g_{\max }\right)}\right) .
$$


Proof. From Lemma 6.10 it follows that $\left(\tilde{\chi}^{\ell}, R^{\ell}(g), r_{g}^{\ell}\right)$ satisfies the KRS properties on tree $\tilde{T}^{\ell}$. Furthermore, using $\bar{y}_{g}^{\ell} \geqslant \frac{1}{2}$ and the coverage property in Lemma 6.10:

$$
\begin{aligned}
\mu_{g}^{\ell} & :=\mathbb{E}\left[\left|\tau^{\ell} \cap R^{\ell}(g)\right|\right]=\sum_{j \in R^{\ell}(g)} z_{j}^{\ell} \\
& =4 \cdot \sum_{j \in R^{\ell}(g)} \tilde{x}_{j}^{\ell} \geqslant 4 \cdot r_{g}^{\ell} \cdot \bar{y}_{g}^{\ell} \geqslant 2 r_{g}^{\ell} .
\end{aligned}
$$

Here we also used Proposition 6.3 that $\operatorname{Pr}\left[j \in \tau^{\ell}\right]=z_{j}^{\ell}$. By applying Lemma 6.5 with $\delta=1 / 2$, we have

$$
\begin{aligned}
\operatorname{Pr}\left[\left|\tau^{\ell} \cap R(g)\right|<r_{g}\right] & \leqslant \exp \left(-\frac{r_{g}^{\ell}}{2\left(2+r_{g}^{\ell}\left(1+\ln \left|R^{\ell}(g)\right|\right)\right)}\right) \\
& \leqslant \exp \left(-\frac{1}{2\left(3+\ln g_{\max }\right)}\right) .
\end{aligned}
$$

This proves Lemma 6.11.

We now show the following lemma.

Lemma 6.12. Consider any group $\mathrm{g} \in \mathcal{G}$ and $\ell \geqslant \ell(\mathrm{g})$. The probability that $\mathrm{P}^{\ell}$ has a total weight of at most $192\left(3+\log g_{\max }\right) \cdot 2^{\ell}$ and covers $g$ is at least $3 / 4$.

Proof. By Proposition 6.3, we know that each edge $e \in \tilde{E}^{\ell}$ is included in $\tau^{\ell}$ with probability $z_{e}^{\ell}=4 x_{e}^{\prime \ell}$. Since for all $e \in \overline{\mathrm{E}}^{\ell}, \bar{x}_{e} \geqslant 1 / 4$, the expected total weight of the edges in $\overline{\mathrm{E}}^{\ell}$ and $\tau^{\ell}$ is upper bounded by

$$
\sum_{e \in \overline{\mathrm{E}}^{\ell}} w_{e}+\sum_{e \in \tilde{\mathrm{E}}^{\ell}} w_{e} \cdot 4 \tilde{x}^{\ell} \leqslant 4 \sum_{e \in \mathrm{E}} w_{e} \cdot \bar{x}_{e}^{\ell} \leqslant 4 \cdot 2^{\ell}
$$

The last inequality is due to the constraints (39). Hence the expected cost of $\mathrm{P}^{\ell}$ is at most $24\left(3+\log g_{\max }\right) \cdot 2^{\ell}$. Markov inequality immediately gives that the total weight of $\mathrm{P}^{\ell}$ is greater than $192\left(3+\log g_{\max }\right) \cdot 2^{\ell}$ with probability at most $1 / 8$. Since $\tau_{\ell}$ is sampled $6\left(3+\log g_{\max }\right) \cdot 2^{\ell}$ times, from Lemma 6.11, we know that group $g$ is not covered by $\mathrm{P}^{\ell}$ with a probability of at most $1 / e^{3} \leqslant 1 / 8$. Hence the claim follows.

Fix any group $\mathrm{g} \in \mathcal{G}$, and $\ell \geqslant \ell(g)$. Among $\mathrm{P}^{\ell(\mathrm{g})}, \mathrm{P}^{\ell(\mathrm{g})+1}, \ldots, \mathrm{P}^{\ell}$, consider the paths that are added to $\pi$. Clearly the total weight of such paths is at most $\mathrm{O}\left(\log g_{\max } \cdot 2^{\ell}\right)$. By Lemma 6.12, the probability that none of these 
paths covers $g$ is at most $\frac{1}{4^{\ell-\ell(g)+1}}$. Hence the expected cover time of $g$ is at most

$$
\sum_{\ell \geqslant \ell(g)} \mathrm{O}\left(\log g_{\max }\right) \cdot 2^{\ell} \cdot \frac{1}{4^{\ell-\ell(g)+1}}=\mathrm{O}\left(\log g_{\max } \cdot 2^{\ell(g)}\right) .
$$

Thus the expected total cover time

$$
\text { ALG-LCST } \leqslant \mathrm{O}\left(\log g_{\max }\right) \cdot \sum_{\mathrm{g} \in \mathcal{G}} 2^{\ell(g)}
$$

By the definition of $2^{\ell(g)}$ being $\frac{1}{2}$-completion time in the LP, we know

$$
\begin{aligned}
\mathrm{OPT} & \geqslant \frac{1}{2} \cdot \sum_{\ell \geqslant 0} 2^{\ell} \sum_{\mathrm{g} \in \mathcal{G}}\left(1-\overline{\mathrm{y}}_{\mathrm{g}}^{\ell}\right) \\
& \geqslant \frac{1}{2} \sum_{\mathrm{g} \in \mathcal{G}} 2^{\ell(g)-1}\left(1-\overline{\mathrm{y}}_{\mathrm{g}}^{\ell(\mathrm{g})-1}\right) \quad \geqslant \frac{1}{8} \cdot \sum_{\mathrm{g} \in \mathcal{G}} 2^{\ell(g)} .
\end{aligned}
$$

These two inequalities show that ALG-LCST is an $\mathrm{O}\left(\log g_{\max }\right)$-approximation on tree instances. By the tree embedding due to [39], we conclude that ALG-LCST can be used to yield an $\mathrm{O}\left(\log \mathrm{g}_{\max } \cdot \log |\mathrm{V}|\right)$-approximation for general metrics, thus proving Theorem 6.1. 

Part II

ROUTING AND CONNECTIVITY 



\section{PATH TRADING}

This chapter is based on the following paper:

A. Berger, H. Röglin and R. van der Zwaan, "Path Trading: Fast Algorithms, Smoothed Analysis, and Hardness Results", in Experimental Algorithms (SEA) 2011. Lecture Notes in Computer Science 6630:43-53, 2011. [15].

\subsection{INTRODUCTION}

The Border Gateway Protocol (BGP) serves as the main routing protocol on the top level of the Internet and ensures network reachability among autonomous systems (ASes). When traffic is forwarded from a source to a destination, these ASes cooperate in order to provide the necessary infrastructure needed to ensure the desired services. However, ASes do also compete and therefore follow their individual strategies and policies when it comes to routing the traffic within their own network. Such locally preferable routing decisions can be globally disadvantageous. Particularly, the way how one AS forwards traffic and through which node another AS may therefore receive the traffic can make a huge difference in the costs for that other AS. Behaving selfishly usually means that an AS routes its traffic according to the least expensive route, also known as hot-potato routing, without regarding the costs of the next AS in the BGP path. This is supported by strong evidence by Teixeira et al. [101].

Quite a number of protocols have been suggested that require the exchange of information and coordination in order to overcome global suboptimality while at the same time improving the costs for each individual AS [77, 79, 105]. Recently, Shavitt and Singer [96] considered the case where ASes might be willing to trade traffic in such a way that the costs for both ASes do not increase w.r.t. the hot-potato routing, and term this problem path trading. They prove that for two ASes the problem of deciding whether there is a feasible path trade is weakly NP-hard. Further, they develop an 
algorithm based on dynamic programming to find the "best" trading between a pair. Lastly, they give experimental evidence that path trading can have benefits to autonomous systems.

In this paper we extend their work in the following way. We show that path trading is also strongly NP-hard when an arbitrary number of ASes is considered. This justifies the approach taken by Shavitt and Singer as well as the approach taken in this paper to concentrate on path trades between pairs of ASes. We propose a new algorithm for finding path trades between pairs of ASes that is based on the concept of Pareto efficiency. We have implemented both, our algorithm and the algorithm of Shavitt and Singer, and tested them on real Internet instances stemming from [95]. Besides the added advantage that our algorithm obtains all Pareto-optimal path trades, it is very fast and has low memory consumption. As the problem is NPhard, we cannot expect that the algorithm performs well on all possible inputs. However, in order to support the experimental results we consider our algorithm in the framework of smoothed analysis, which was introduced in 2001 by Spielman and Teng [100] to explain why many heuristics with a bad worst-case performance work well on real-world data sets. We show that even though there are (artificial) worst-case instances on which the heuristic performs poorly, it has a polynomial expected running time on instances that are subject to small random perturbations. After its introduction, smoothed analysis has been applied in many different contexts (see [99] for a nice survey).

Finding path trades can be viewed as an optimization problem with multiple objectives that correspond to the costs of the different ASes. A feasible path trade is then a solution that is in every objective at least as good as the hot-potato routing. We say that such a path trade dominates the hot-potato routing if it is strictly better in at least one objective. This brings us to the well-known concept of Pareto efficiency or Pareto optimality in multiobjective optimization: A solution is called Pareto-optimal if it is not dominated by any other solution, that is, a solution is Pareto-optimal if there does not exist another solution that is at least as good in all criteria and strictly better in at least one criterion. We call the set of Pareto-optimal solutions Pareto set or Pareto curve for short.

Then the question of whether there is a feasible path trade can simply be formulated as the question whether the hot-potato routing is Paretooptimal or not. This immediately suggests the following algorithm to find a feasible path trade: Enumerate the set of Pareto-optimal solutions, and then either output that there is no path trade if the hot-potato routing be- 
longs to the Pareto set, or output a Pareto-optimal solution that dominates the hot-potato routing if it is not Pareto-optimal. Also, finding the Pareto set gives the flexibility to choose a solution based on preference. While some solutions might offer great global gain, these trade-offs might be unreasonable from a fairness perspective.

The aforementioned algorithm only works when the Pareto set is small because otherwise the computation becomes too time consuming. Our experiments show that the number of Pareto-optimal path trades is indeed small and that despite the NP-hardness result by Shavitt and Singer we can solve this problem efficiently in practice for two ASes.

For path trading between an arbitrary number of ASes, however, there is little hope for obtaining such a result: We show that our strong NPhardness result implies that this problem cannot be solved efficiently even in the framework of smoothed analysis.

RELATED WORK. The potential benefits of collaboration between neighboring ASes and the necessary engineering framework were first introduced by Winick et al. [105]. They consider the amount of information that needs to be shared between the ASes in order to perform mutually desirable path trades and how to limit the effect of path trades between neighboring ASes on the global flow of traffic. The first heuristics for path trading to improve the hot-potato routing were evaluated by Majahan et al. [79]. Majahan et al. also developed a routing protocol that provides evidence that path trading can improve global efficiency in Internet routing. Other related work in the area of improving the global performance while maintaining the objectives of the different ASes has been done by Yang et al. [108], Liu and Reddy [77], and by Quoitin and Bonaventure [87]. Since ASes usually compete, one cannot expect them to reveal their complete network and cost structure when it comes to coordinating the traffic between the ASes. This aspect is considered in the work by Shrimali et al. [97], using aspects from cooperative game theory and the idea of Nash bargaining. Goldenberg et al. [48] develop routing algorithms in a similar context to optimize global cost and performance in a multihomed user setting, which extends previous work in that area $[1,34,94]$. 


\subsection{MODEL AND NOTATION}

The model is as follows. We have the Internet graph $G=(V, E)$, where every vertex represents a point/IP-address. Further, there are $k$ ASes and the vertex set $V$ is partitioned into mutually disjoint sets $V_{1}, \ldots, V_{k}$, where $V_{i}$ represents all points in AS $i$. We denote by $E_{i}$ all edges within AS $i$, that is, the set of edges $E$ is partitioned into $E_{1}, \ldots, E_{k}$, and the set of edges between different ASes. The graph $\mathrm{G}$ is undirected, and each edge $e \in \mathrm{E}$ has a length $\ell(e) \in \mathbb{R}_{\geqslant 0}$. The traffic is modeled by a set of requests $R$, where each request is a triple $(s, t, c)$, where $s \in V$ and $t \in V$ are source and sink nodes, respectively, and $c \in \mathbb{R}_{\geqslant 0}$ is the cost of the corresponding request. The BGP protocol associates with each request a sequence of ASes which specifies the order in which the request has to be routed through the ASes. Since most of the paper is about the situation between two ASes, we leave this order implicit. The cost of routing a request with cost $c$ through edge $e$ is $\ell(e) \cdot c$. For simplicity, the costs of routing a packet between two ASes are assumed to be zero, but each request can be routed at most once from an AS to the next AS. The input for PATH Trading consists of the graph G and requests as described previously. We denote by $n$ the number of nodes in $\mathrm{V}$.

For a given graph $\mathrm{G}$ and a request $(s, \mathrm{t}, \mathrm{c})$ we say that a path $\mathrm{P}$ is valid if it connects $s$ to $t$ and visits the ASes in the order that is associated with this request by the BGP protocol. This means, in particular, that every valid path goes through every AS at most once. A solution to PATH Trading is a mapping that maps each request $(s, t, c) \in R$ to a valid path from $s$ to $t$ in graph G. Let us assume that the requests in $R$ are $\left(s_{1}, t_{1}, c_{1}\right), \ldots,\left(s_{r}, t_{r}, c_{r}\right)$ and that the paths $P_{1}, \ldots, P_{r}$ have been chosen for these requests. Then AS $i$ incurs costs on all edges in $E_{i}$, i.e., it incurs a total cost of

$$
\sum_{j=1}^{r}\left(c_{j} \cdot \sum_{e \in P_{j} \cap E_{i}} \ell(e)\right) .
$$

The hot-potato route of a request $(s, t, c)$ is defined to be the concatenation of shortest path routes for the ASes it goes through. To be precise, assume that the BGP protocol associates the route $i_{1}, \ldots, i_{m}$ with $s \in V_{i_{1}}$ and $t \in$ $V_{i_{m}}$ with this request. Then AS $i_{1}$ sends the request from $s$ to the vertex $s_{2} \in V_{i_{2}}$ that is closest to $s$ along the shortest path. Then AS $i_{2}$ sends the request from $s_{2}$ to the vertex $s_{3} \in V_{i_{3}}$ that is closest to $s_{2}$ along the shortest path, and so on. The complete hot-potato route for request $(s, t, c)$ 
is then the concatenation of these paths. Note that the hot-potato route is not necessarily unique, and in the following we will assume that some hot-potato route is chosen for each request.

Consequently, the costs of the hot-potato routing that an AS $i$ incurs are equal to Equation 42 , where the paths $\mathrm{P}_{1}, \ldots, \mathrm{P}_{\mathrm{r}}$ are the hot-potato paths. We call a solution to PATH TRADING a path trade and if the costs for all involved ASes are less or equal to their hot-potato costs, then we call it a feasible path trade. In the following, let $[n]$ be the set of integers $\{1, \ldots, n\}$. For a vector $x \in \mathbb{R}^{\mathfrak{n}}$, let $x_{i}$ be the $i$-th component of $x$.

\subsection{COMPLEXITY RESULTS AND SMOOTHED ANAL- YSIS}

Our first result is about the complexity of PATH TRADING and extends the weak NP-hardness result of Shavitt and Singer [96]. The proof uses a reduction from 3-PARTITION.

Theorem 7.1. Finding a feasible path trade apart from the hot-potato routing is strongly NP-hard.

Proof. We will prove the statement by a reduction from the following version of 3-PARTITION. An instance of 3-PARTITION consists of a multiset $S=\left\{b_{1}, \ldots, b_{3 m}\right\}$ of $3 m$ positive integers, where $\sum_{i=1}^{3 m} b_{i}=m W$ for some integer $W$ and for every $i$ it holds that $W / 4<b_{i}<W / 2$. The problem is to determine whether there exists a partition of $S$ into $m$ subsets of size three such that the elements in every subset add up to $W$. This problem is strongly NP-hard, i.e. it is NP-hard even if all the numbers $b_{i}$ are bounded by a polynomial in $\mathrm{m}$.

From an instance for 3-PARTITION we will construct an instance of PATH TRADING such that there exists a feasible path trade apart from the hotpotato routing in that instance if and only if there exists a solution to the instance of 3-PARTITION.

The instance will consist of $m+2$ ASes $A, B$, and $A_{1}, \ldots, A_{m}$. There is one request $(x, y, 1)$ where $x \in A$ and $y \in B$, which has to be routed through the ASes in the order $A, A_{1}, \ldots, A_{m}, B$. In the AS $A$ the node $x$ is connected to two boundary nodes $x_{0}$ and $x_{1}$, where the weight of the edge $x x_{0}$ is 0 and the weight of the edge $x x_{1}$ is $m W$. Moreover, the node $y$ in the AS B is connected to two boundary nodes $y_{0}$ and $y_{1}$ with edges of 


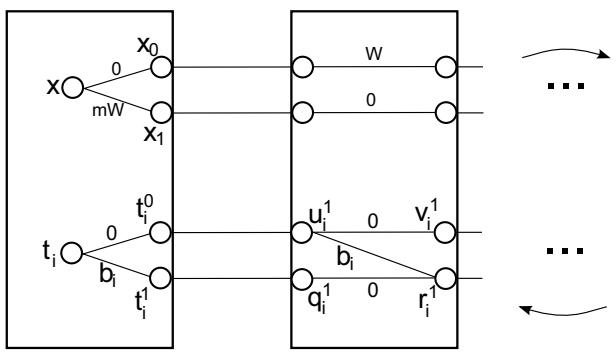

A

$A_{1}$

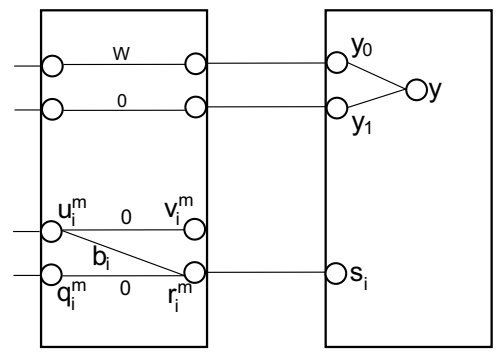

$A_{m}$

B

Figure 11: This figure depicts the reduction from 3-PARTITION to PATH TRAdING in Theorem 7.1.

weight $\mathrm{o}$. In addition, there is a unique path that connects $x_{0}$ and $y_{0}$ going through the ASes $A_{1}, \ldots, A_{m}$ and using one edge of weight $W$ in each AS, and there is a unique path that connects $x_{1}$ and $y_{1}$ going through the ASes $A_{1}, \ldots, A_{m}$ and using one edge of weight $o$ in each AS.

We note that the request $(x, y)$ can only be routed in two different ways: under the hot-potato strategy it is routed via $x_{0}$ and $y_{0}$ and $A$ incurs a cost of $o$, while each $A_{j}$ incurs a cost of $W$. The second option is to route the request via $x_{1}$ and $y_{1}$ with a cost of $m W$ for $A$ and a cost of o for each $A_{j}$.

In addition to the above request, there will be $3 \mathrm{~m}$ additional requests, each responding to one element in the multiset $S$. For each $b_{i} \in S$ there will be a request $\left(s_{i}, t_{i}, 1\right)$, where $s_{i} \in B$ and $t_{i} \in A$ that has to be routed in the order $B, A_{m}, \ldots, A_{1}, A$. For each AS $A_{j}$, there will be four nodes $q_{i}^{j}, r_{i}^{j}, u_{i}^{j}, v_{i}^{j} \in A_{j}$ together with three edges: $\left\{q_{i}^{j}, r_{i}^{j}\right\}$ and $\left\{u_{i}^{j}, v_{i}^{j}\right\}$ of weight $o$ and $\left\{u_{i}^{j}, r_{i}^{j}\right\}$ of weight $b_{i}$. For $1 \leqslant j \leqslant m-1, v_{i}^{j}$ is connected to $u_{i}^{j+1}$ and $r_{i}^{j}$ is connected to $q_{i}^{j+1}$. Finally, $t_{i}$ is connected to $u_{i}^{1}$ with an edge of weight $o$ via the node $t_{i}^{0}$ and to $q_{i}^{1}$ with an edge of weight $b_{i}$ via the node $t_{i}^{1}$, and $s_{i}$ is connected to $r_{i}^{m}$. See Figure $I$ for an overview of the nodes, edges, and requests.

Under the hot-potato strategy any request $\left(s_{i}, t_{i}\right)$ is routed through the nodes $s_{i}, r_{i}^{m}, q_{i}^{m}, \ldots, r_{i}^{1}, q_{i}^{1}, t_{i}^{1}, t_{i}$ for a cost of o for each $A_{j}$ and for a cost of $b_{i}$ for $A$. Thus the total cost of all requests $\left(s_{i}, t_{i}\right)$ for AS $A$ is $m W$. Each request $\left(s_{i}, t_{i}\right)$ can also be "rerouted in $A_{j}$ " by using the edge $\left\{r_{i}^{j}, u_{i}^{j}\right\}$. This increases the cost of $A_{j}$ by $b_{i}$ and decreases the cost of $A$ by $b_{i}$ when compared to the hot-potato strategy. It is straightforward to see that in any reasonable path trade every request $\left(s_{i}, t_{i}\right)$ goes through at most one edge 
$\left\{r_{i}^{j}, u_{i}^{j}\right\}$ because otherwise the costs for some ASes can be decreased without increasing the costs for other ASes.

In order to finish the proof, we first assume that there exists a solution $S_{1}, \ldots, S_{m}$ to the instance of 3 -PARtition, i.e. $\bigcup_{j=1}^{m} S_{j}=S$ and $\sum_{b_{i} \in S_{j}} b_{i}=$ $W$ for every $1 \leqslant j \leqslant m$. The corresponding path trading solution that deviates from the hot-potato strategy is to route the request $(x, y)$ via $x_{1}$ and $y_{1}$ with an increase in cost of $m W$ for $A$ and a decrease in cost of $W$ for each $A_{j}$. Moreover, each request $\left(s_{i}, t_{i}\right)$ is rerouted in that $A_{j}$ for which $b_{i} \in S_{j}$. Thus each $A_{j}$ saves a cost $W$ from the rerouting of $(x, y)$ and has an increase in cost of $\sum_{b_{i} \in S_{j}} b_{i}=W$. Moreover, since each request $\left(s_{i}, t_{i}\right)$ is rerouted in some $A_{j}$, the AS A will save a total of $m W$ in cost, but has an increase in cost of $\mathrm{mW}$ from the rerouting of $(x, y)$. Thus this is a feasible rerouting scheme.

Finally, assume that there exists a feasible rerouting scheme of all requests. If the request $(x, y)$ is rerouted, then certainly there is an increase in cost of $m W$ for $A$ and a decrease in cost of $W$ for each $A_{j}$. Since the rerouting scheme is feasible, the only way for $A$ to also save at least $\mathrm{mW}$ in cost is to have all requests $\left(s_{i}, t_{i}\right)$ be routed to $t_{i}$ via $t_{i}^{0}$, i.e. each $\left(s_{i}, t_{i}\right)$ has to be rerouted in some $A_{j}$. We let $S_{j}=\left\{b_{i}: b_{i}\right.$ is rerouted in $\left.A_{j}\right\}$. Since each $A_{j}$ has saved a cost of $W$ through the rerouting of $(x, y)$, we have that $\sum_{b_{i} \in S_{j}} b_{i} \leqslant W$ for each $j$ because otherwise the costs for $A_{j}$ would be larger than in the hot-potato routing. Due to the premise that $W / 4<b_{i}<W / 2$ for all $i$ the only way to achieve all the reroutings while maintaining feasibility for all the ASes $A_{j}$ is if $\left|S_{j}\right|=3$ and $\sum_{b_{i} \in S_{j}} b_{i}=W$ for all $j$. Thus we get a solution to the instance of 3-Partition in this case. Now, if on the other hand one request $\left(s_{i}, t_{i}\right)$ is rerouted in some $A_{j}$, then $A_{j}$ has an additional cost of $b_{i}$ and since the only way for $A_{j}$ to save cost is to reroute $(x, y)$, we are back in the first case. This finishes the proof.

Note, that the edges of length $W, \mathrm{~mW}$, and $b_{i}$ can be replaced by paths of $W, m W$, and $b_{i}$ edges of length 1 , respectively, which shows that the version of path trading where edges are only allowed to have length 0 or 1 is also strongly NP-hard.

Given the above theorem, in order to develop fast algorithms, we concentrate on path trading between two ASes, and will now present our algorithm for this case. As mentioned before, this algorithm is based on the concept of Pareto efficiency and it enumerates all Pareto-optimal path trades. In the worst case the number of Pareto-optimal solutions can be exponential, but our experiments suggest that on real-world data usually 
only a few solutions are Pareto-optimal. To give a theoretical explanation for this, we apply the framework of smoothed analysis. The algorithm is a dynamic program that adds the requests one after another, keeping track of the Pareto-optimal path trades of those requests that have already been added.

For a request $(s, t, c)$, a path $P$ from $s$ to $t$, and $i \in\{1,2\}$, we denote by $C_{i}(P)$ the costs incurred by AS $i$ due to routing the request along path $P$. To keep the notation simple, assume in the following discussion w.l.o.g. that $s \in V_{1}$ and $t \in V_{2}$. We denote by $\mathcal{P}(s, t)$ the set of all Pareto-optimal valid paths from $s$ to $t$. Remember that a path is valid if it starts at $s$, terminates at $t$, and does not go back to $V_{1}$ after leaving $V_{1}$ for the first time. Such a path $P$ belongs to $\mathcal{P}(s, t)$ if there does not exist another valid path that induces strictly lower costs for one AS and not larger costs for the other AS than P. We assume that in the case that there are multiple paths that induce for both ASes exactly the same costs, only one of them is contained in $\mathcal{P}(s, t)$.

Let $\mathrm{P} \in \mathcal{P}(s, t)$ be some Pareto-optimal path and let $v \in \mathrm{V}_{1}$ be the boundary node at which the path leaves AS 1. Then the subpaths from $s$ to $v$ and from $v$ to $t$ must be shortest paths in AS 1 and AS 2, respectively. Otherwise, $\mathrm{P}$ cannot be Pareto-optimal. Hence, the number of Pareto-optimal paths in $\mathcal{P}(s, t)$ is bounded from above by the number of boundary nodes of AS 1 that connect to AS 2. For each pair $s \in V_{1}$ and $t \in V_{2}$, the $\operatorname{set} \mathcal{P}(s, t)$ can be computed in polynomial time.

Our algorithm first computes the set $\mathcal{P}_{1}$ of Pareto-optimal path trades for only the first request $\left(s_{1}, t_{1}, c_{1}\right)$. This is simply the set $\mathcal{P}\left(s_{1}, t_{1}\right)$. Based on this, it computes the set $\mathcal{P}_{2}$ of Pareto-optimal path trades for only the first two requests, and so on. Thus the elements in $\mathcal{P}_{i}$ are tuples $\left(P_{1}, \ldots, P_{i}\right)$ where each $P_{j}$ is a valid path for the $j$ th request.

Theorem 7.2. For $i \in[r]$, the set $\mathcal{P}_{i}$ computed by Algorithm 1 is the set of Paretooptimal path trades for the first $i$ requests. In particular, the set $\mathcal{P}_{\mathrm{r}}$ is the set of Pareto-optimal path trades for all requests. Algorithm 1 can be implemented to run in time $\mathrm{O}\left(\mathrm{n} \log \mathrm{n} \cdot \sum_{i=1}^{r}\left|\mathcal{P}_{\mathfrak{i}}\right|+n \mathrm{n}|\mathrm{E}| \log \mathrm{n}\right)$

Proof. Let us first argue that $\mathcal{P}_{i}$ is the set of Pareto-optimal path trades for the first $i$ requests. For $i=1$, it follows from the definition that $\mathcal{P}_{1}=$ $\mathcal{P}\left(s_{1}, t_{1}\right)$ is the set of Pareto-optimal path trades for the first request. Now given a Pareto-optimal path trade $\left(P_{1}, \ldots, P_{i}\right)$ for $i>1$, one can easily argue that $P_{i}$ must be from the set $\mathcal{P}\left(s_{i}, t_{i}\right)$ because otherwise, if it was dominated by some other path $Q \in \mathcal{P}\left(s_{i}, t_{i}\right)$, then $\left(P_{1}, \ldots, P_{i-1}, Q\right)$ would 


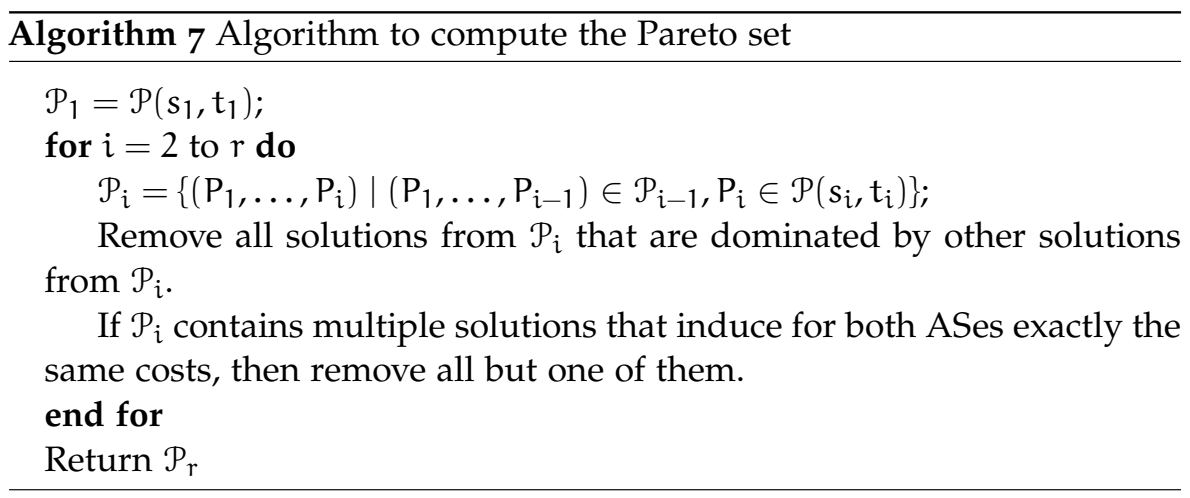

dominate $\left(\mathrm{P}_{1}, \ldots, \mathrm{P}_{i}\right)$, contradicting the assumption that $\left(\mathrm{P}_{1}, \ldots, \mathrm{P}_{i}\right)$ is a Pareto-optimal path trade. By exactly the same argument one can argue that $\left(P_{1}, \ldots, P_{i-1}\right)$ has to be a Pareto-optimal path trade for the first $i-1$ requests. Hence, after the inner for loop in Algorithm 1 , the set $\mathcal{P}_{i}$ is a superset of the set of Pareto-optimal path trades of the first $i$ requests. In the following two steps only dominated solutions are removed and for every cost vector only one solution inducing it is kept. This guarantees that afterwards the set $\mathcal{P}_{i}$ contains exactly the Pareto-optimal path trades of the first $i$ requests. The most straightforward implementation of the algorithm would yield a running time that is polynomial in $\mathrm{n}$ and the cardinalities of $\mathcal{P}_{i}$, but the degree of this polynomial would be larger than the one claimed. We will argue now that an implementation reminiscent of the Nemhauser/Ullmann algorithm for the knapsack problem [85] achieves the claimed running time. For this, first of all observe that for each request the set $\mathcal{P}\left(s_{i}, t_{i}\right)$ can be computed by at most $2 n$ shortest path computations as there are at most $n$ boundary nodes. Each of these calculations can be done, e.g., by Dijkstra's algorithm in time $\mathrm{O}(|\mathrm{E}| \log n)$, yielding a total running time of $O(n r|E| \log n)$ for the shortest path calculations.

Now assume that all sets $\mathcal{P}\left(s_{i}, t_{i}\right)$ have been computed. In order to speed up the algorithm we will maintain the following invariant: when iteration $i$ of the outer for loop starts, the set $\mathcal{P}_{i-1}$ is stored in a linked list that is sorted in increasing order of $\mathrm{C}_{1}(\mathrm{P})$. That is, we keep $\mathcal{P}_{i-1}$ sorted according to the costs of AS 1. Now let us look at the inner for loop. This for loop creates for each path $\mathrm{P} \in \mathcal{P}\left(s_{i}, t_{i}\right)$ a copy of $\mathcal{P}_{i-1}$ and adds $\mathrm{P}$ to all elements of this copy. Hence, if $\mathcal{P}_{\mathfrak{i}-1}$ is sorted, then without additional effort also these copies are sorted. The total time that the inner for loop takes is $\mathrm{O}\left(\left|\mathcal{P}_{i-1}\right| \cdot\left|\mathcal{P}\left(s_{i}, t_{i}\right)\right|\right)=\mathrm{O}\left(n \cdot\left|\mathcal{P}_{i-1}\right|\right)$. 
Now we have at most $n$ copies of $\mathcal{P}_{i-1}$ and we have to merge them into a single sorted list. Once this sorted list is computed, duplicates and dominated solutions can be removed by a single linear scan. Merging $n$ sorted lists of length $\left|\mathcal{P}_{i-1}\right|$ each can be accomplished in time $\mathrm{O}(n \log n$. $\left.\left|\mathcal{P}_{i-1}\right|\right)$ by standard merging algorithms [75]. Adding up the running times for the iterations of the outer for loop yields the claimed bound.

We start by reviewing a result due to Beier et al. [13] who analyzed the number of Pareto-optimal solutions in binary optimization problems with two objective functions. They consider problems whose instances have the following form: the set of feasible solutions $S$ is a subset of $\{0, \ldots, F\}^{n}$ for some integers $F$ and $n$, and there are two objective functions $w^{(1)}: S \rightarrow \mathbb{R}$ and $w^{(2)}: S \rightarrow \mathbb{R}$ that associate with each solution $x \in S$ two weights $w^{(1)}(x)$ and $w^{(2)}(x)$ that are both to be minimized. While $w^{(2)}$ can be an arbitrary function, it is assumed that $w^{(1)}$ is linear of the form $w^{(1)}(x)=$ $w_{1} x_{1}+\ldots+w_{n} x_{n}$.

In a worst-case analysis, the adversary would be allowed to choose the set of feasible solutions $S$, and the two objective functions $w^{(1)}$ and $w^{(2)}$. Then it can easily be seen that there are choices such that the number of Pareto-optimal solutions is exponential. To make the adversary less powerful and to rule out pathological instances, we assume that the adversary cannot choose the coefficients $w_{1}, \ldots, w_{n}$ exactly. Instead he can only specify a probability distribution for each of them according to which it is chosen independently of the other coefficients. Without any restriction, this would include deterministic instances as a special case, but we allow only probability distributions that can be described by a density function that is upper bounded by some parameter $\phi \geqslant 1$.

We denote by $f_{i}: \mathbb{R}_{\geqslant 0} \rightarrow[0, \phi]$ the probability density according to which $w_{i}$ is chosen, and we assume that the expected value of $w_{i}$ is in $[0,1]$.

Theorem 7.3 (Beier et al., [13]). For any choice of feasible solutions $\mathcal{S} \subseteq\{0, \ldots, F\}^{n}$, any choice of $w^{(2)}$ and any choice of density functions $f_{1}, \ldots, f_{n}$, the expected number of Pareto-optimal solutions is bounded by $\mathrm{O}\left(\phi \mathrm{n}^{2} \mathrm{~F}^{2} \log \mathrm{F}\right)$.

Now we formulate our problem in terms of Theorem 7.3. For this, we assume that all requests have positive integer costs. Let $F$ denote an upper bound on the maximal costs possible on any edge, e.g., $F=\sum(s, t, c) \in R$ $m=|E|$ and assume that the edges in $E$ are ordered arbitrarily. Then each path trade leads to a cost vector $x \in\{0, \ldots, F\}^{m}$ where $x_{1}$ denotes the total cost of all requests that use the first edge in $E, x_{2}$ denotes the total cost 
of all requests that use the second edge in $E$, and so on. If two solutions lead to the same cost vector, then it suffices to remember one of them, and hence, we can assume that the set of possible path trades can essentially be described by the set $S \subseteq\{0, \ldots, F\}^{m}$ of possible cost vectors. Given such a cost vector $x \in\{0, \ldots, F\}^{m}$, we can express the cost $w^{(1)}(x)$ of the first AS simply as $\sum_{e \in \mathrm{E}_{1}} \ell(e) x_{e}$. The costs of the second AS can be defined analogously, and so it looks like that Theorem 7.3 directly applies when we perturb all edge lengths $\ell(e)$ of edges $e \in E_{1}$ as these edge lengths are the coefficients in the linear objective function $w^{(1)}$. However, there is a small twist. In Theorem 7.3 all coefficients in the linear objective function $w^{(1)}$ are chosen randomly. Our objective function, however, does not contain terms for the edges $e \in \mathrm{E}_{2}$. Or with other words the coefficients are 0 for these edges. If we apply Theorem 7.3 directly, then also these zero coefficients would be perturbed, which would destroy the combinatorial structure of the problem as then suddenly the cost of the first AS would depend on what happens on edges $e \in E_{2}$.

To avoid this side effect, we remodel the feasible region $S$. As argued before, each solution leads to a cost vector $x \in\{0, \ldots, F\}^{m}$, but now we care only about the part of the vector for $E_{1}$. Let us define $m^{\prime}=\left|E_{1}\right| \leqslant m$. Then each solution leads to a cost vector $x \in\left\{0, \ldots, \mathrm{F}^{\mathrm{m}^{\prime}}\right.$ that contains only the costs of the first AS. Now of course different solutions can lead to the same vector $x$ if they differ only in the way how the traffic is routed in the second AS. However, Theorem 7.3 allows completely general objective functions $w^{(2)}$, which we exploit by defining $w^{(2)}(x)$ for a vector $x \in\{0, \ldots, F\}^{m^{\prime}}$ to be the smallest cost for the second AS that can be achieved with any solution whose cost vector for the first AS results in $x$. This formulation implies the following corollary.

Corollary 7.4. Given a path trading instance in which the edge lengths $\ell(e)$ for all $e \in \mathrm{E}_{1}$ are randomly chosen according to probability distributions that satisfy the same restrictions as those in Theorem 7.3, the expected number of Pareto-optimal solutions is bounded by $\mathrm{O}\left(\phi \mathrm{m}^{2} \mathrm{~F}^{2} \log \mathrm{F}\right)$.

Given that the expected number of Pareto-optimal solutions is small, we still have to show that Algorithm I computes the Pareto curve in expected polynomial time. This will be established by the following Corollary.

Corollary 7.5. Algorithm 1 computes the Pareto curve in expected time $\mathrm{O}\left(\phi \mathrm{nm}^{2}\right.$. $\left.\log n \cdot r F^{2} \log F\right)$. 
Proof. The corollary follows immediately from Theorem 7.2 and Corollary 7.4 when we observe that Corollary 7.4 does not only bound the expected number of Pareto-optimal path trades for all requests, but that it also bounds for each $i$ the expected number of Pareto-optimal path trades for requests $1, \ldots, i$. Then linearity of expectation implies that the running time is bounded by $\mathrm{O}\left(n \log n \cdot \sum_{i=1}^{r} E\left(q_{i}\right)+n r|E| \log n\right)=O\left(\phi n m^{2} \log n\right.$. $\left.r F^{2} \log F\right)$.

The reason that we concentrate our efforts on path trading between two ASes was the hardness result in Theorem 7.1. We can extend this result and also show that there is no hope for PATH TRADING with an arbitrary number of ASes to be solvable efficiently in the framework of smoothed analysis.

Theorem 7.6. There is no smoothed polynomial time algorithm for PATH TRADING with an arbitrary number of ASes, unless NP $\subseteq$ BPP.

Proof sketch. We only present the main idea of the proof. Assume that we have an algorithm $A$ for PATH TRADING with an arbitrary number of ASes whose running time in the model of smoothed analysis is bounded with constant probability by a polynomial in the input size and the parameter $\phi$. We claim that we can utilize this algorithm to solve the instances in Corollary 7.7 with constant probability in polynomial time. This would immediately imply that 3-PARTITION is in BPP.

The main idea is that the instances of PATH TRADING that we construct in Corollary 7.7 are rather robust against small perturbations of the edge lengths and satisfy the following two properties. If there exists a solution to the given 3-PARTITION instance, there exists a feasible path trade in which the costs for each AS are smaller by at least some constant c compared to the hot-potato routing. If there does not exist a solution to the given 3PARTITION instance, any path trade deviating from the hot-potato routing increases the costs of at least one AS by at least a constant $c^{\prime}$ and is hence infeasible. Now we consider the smoothed scenario in which we cannot precisely specify the edge lengths anymore, but only choose a probability density for each of them. We choose $\phi$ polynomially large in $m$ and assume that every edge length is chosen according to a uniform distribution from an interval of length $1 / \phi$ around the value that it gets in the reduction in Corollary 7.7. Due to the aforementioned two properties, an instance generated at random according to these density functions still satisfies the property that there is a feasible path trade that is cheaper for each AS 
than the hot-potato routing if and only if there exists a solution to the 3PARTITION instance if $\phi$ is sufficiently large. This is because the uncertainty induced by the random choices of the edge lengths is smaller than the constants $c$ and $c^{\prime}$.

Hence, we can solve 3-PARTITION as follows: apply the aforementioned randomized reduction to the given instance, and then run algorithm $A$ for polynomially many steps. If it stops, which happens with constant probability, then we know whether or not there exists a solution to the given 3-PARTITION instance. Otherwise, we output yes or no with probability $1 / 2$ each. This algorithm outputs the correct solution with constant probability larger than $1 / 2$.

Corollary 7.7. It is strongly NP-hard to decide whether there exists a feasible path trade that is for all ASes strictly better than the hot-potato routing.

Proof. We use the same reduction as in the proof of Theorem 7.I and adjust edge lengths as follows: We set $\ell\left(x x_{1}\right)=m W-1 / 2$, and for all $i \leqslant 3 m$ and for all $j \leqslant m$, we set $\ell\left(r_{i}^{j} u_{i}^{j}\right)=b_{i}-\frac{1}{5}$. Additionally, we set $\ell\left(y_{0} y\right)=1$. It is easy to see that a 3-partition still gives rise to a feasible path trade in the same way as in Theorem 7.1. In this path trade, AS A reduces its costs by $1 / 2$ compared to the hot-potato routing, every AS $A_{j}$ reduces its costs by $3 / 5$, and $B$ reduces its costs by 1 .

To argue that any valid path trade apart from the hot-potato routing gives rise to a 3-partition, observe that in any such path trade, request $(x, y)$ has to be rerouted as otherwise none of the other requests can be rerouted either. So assume that request $(x, y)$ is rerouted. Then every request $\left(s_{i}, t_{i}\right)$ has to be rerouted in one of the ASes $A_{1}, \ldots, A_{m}$ as otherwise, AS $A$ would incur increased costs compared to the hot-potato routing.

Again let $S_{j}=\left\{b_{i}: b_{i}\right.$ is rerouted in $\left.A_{j}\right\}$. Since all $b_{i}$ are positive integers, the condition that $W / 4<b_{i}$ implies that $b_{i}-W / 4 \geqslant 1 / 4$ and hence $W / 4<$ $b_{i}-1 / 5$. As $\sum_{b_{i} \in S_{j}}\left(b_{i}-1 / 5\right) \leqslant W$ for each $j$, this implies that each $S_{j}$ can contain at most three elements. As there are $3 \mathrm{~m}$ elements in total, this implies that each $S_{j}$ contains exactly three elements. Since $\sum_{b_{i} \in S_{j}}\left(b_{i}-\right.$ $1 / 5)=\left(\sum_{b_{i} \in S_{j}} b_{i}\right)-3 / 5 \leqslant W$ and every $b_{i}$ is integer, we also have that $\sum_{b_{i} \in S_{j}} b_{i} \leqslant W$. As the total sum of elements is $m W$, this implies that for every $j$, we have $\sum_{b_{i} \in S_{j}} b_{i}=W$. 


\subsection{EVALUATION}

In this section we present the experimental results about the performance of our algorithm on the IP-level Internet graph from DIMES [95]. We compare it, in particular, with the performance of the dynamic program used by Shavitt and Singer, and we answer the following questions:

- (Running time) How fast can we compute the Pareto curve? Algorithm I is very fast and scales well.

- (Participation) How many ASes are involved in path trading? In our experiments we see that almost all ASes can benefit from path trading

- (Gain) How much do ASes gain by trading? The gains are typically modest, around $5 \%$.

The answers to these questions are, of course, depending on the assumptions we made. As Shavitt and Singer, we assume that traffic is symmetric, i.e., the number of requests sent from AS $A$ to $A S B$ is the same as the number of requests sent from AS B to AS A for every pair of ASes. This assumption is not necessarily true, but it is common and used, e.g., in [96], [79], and [97]. One could imagine that in real networks requests are not evenly spread, but are more concentrated between popular ASes for example. However, we can still see from our experiments that ASes have a good chance of gaining from path trading as long as there is non-zero traffic in both directions. Our second assumption is that each request between two ASes has to be routed between two nodes that are chosen uniformly at random from these ASes. Our third assumption is that all edges have length 1, i.e., the number of hops is used to measure the costs of an AS for routing a request. By absence of real data, we feel that this a reasonable and common assumption.

In the following subsection we present the details of the experimental setup. The algorithm by Shavitt and Singer is named Algorithm 2. Then we show and discuss the experimental results.

\subsubsection{Experimental Setup}

The test set-up for answering the questions differs slightly, and were performed on different data sets from DIMES. 
EXPERIMENTAL SET-UP FOR "RUNNING TIME" To determine AS pairs where there is potential for path trading and therefore a larger set of Paretooptimal solutions, we simulated a low number of requests for each sufficiently connected pair. To measure how many ASes could benefit from path trading, we simulated 5 requests for each of the 4348 sufficiently connected AS pairs in either direction. For comparing performance we selected a subset of 15 AS pairs arbitrarily among the AS pairs that benefited from path trading.

For both algorithms, we need to calculate shortest paths beforehand. Because of the large number of possible routings, many shortest paths need to be computed. This was all done as part of the preprocessing, and all shortest paths were stored in a hash-table for fast access for both algorithms. In the following, this time is not included in the running times of the algorithms.

EXPERIMENTAL SET-UP FOR "PARTICIPATION" AND "GAIN" The data set is very large. Therefore, computing for for all pairs of ASes how much could be gained from path trading has some bottlenecks. The first bottleneck is the computation of shortest paths, for large graphs the standard algorithms such as Dijkstra run out of memory or are too slow. Since the development of fast algorithms for shortest paths in Internet graphs is out of scope of this paper, we used some other techniques to make this calculation feasible.

Consider the network of a single AS. We call a node a border node if it shares edges with nodes outside its AS. For every pair of ASes, we select 100 nodes uniformly at random that are in both ASes and requests from an AS to another AS can only go through these nodes. Secondly, from all other border nodes we select 1000 nodes uniformly at random where requests can originate from. These are reasonable restrictions since the goal of the experiment is to see how path trading performs on real-life topologies of ASes.

Table 12 summarizes the characteristics of the network for these experiments.

\subsubsection{Experimental Results}

\section{Running time}

Table 1 shows a comparison of the running times of Algorithm 1 and Algorithm 2. The running times are the total of the running time over the 15 


\begin{tabular}{rrr}
\multicolumn{3}{c}{ Size } \\
\hline Low & $\boldsymbol{U} p$ & \# ASes \\
\hline 0 & 0 & 77 \\
1 & 2 & 2210 \\
3 & 5 & 4769 \\
6 & 10 & 4123 \\
11 & 25 & 4745 \\
26 & 100 & 5590 \\
101 & 200 & 1981 \\
201 & 500 & 1635 \\
501 & 1000 & 654 \\
1001 & 2000 & 383 \\
$2001+$ & & 420 \\
\hline
\end{tabular}

(a) The number of nodes in each AS, where \# ASes indicates how many ASes have at least Low and at most $\boldsymbol{U} p$ nodes.

\begin{tabular}{rrr}
\multicolumn{4}{c}{ Border size } \\
\hline Low & $\boldsymbol{U} \boldsymbol{p}$ & \# ASes \\
\hline 0 & 0 & 897 \\
1 & 2 & 4779 \\
3 & 5 & 5456 \\
6 & 10 & 4253 \\
11 & 25 & 4506 \\
26 & 100 & 4209 \\
101 & 200 & 1084 \\
201 & 500 & 760 \\
501 & 1000 & 317 \\
1001 & 2000 & 165 \\
$2001+$ & & 161 \\
\hline
\end{tabular}

(b) The number of outgoing edges from ASes, where \# ASes indicates how many ASes have at least Low and at most $U p$ outgoing edges.

\begin{tabular}{rrr}
\multicolumn{3}{c}{ Shared border size } \\
\hline Low & $\boldsymbol{U} \boldsymbol{p}$ & \# AS pairs \\
\hline 0 & 0 & 872 \\
1 & 2 & 32525 \\
3 & 5 & 16188 \\
6 & 10 & 10064 \\
11 & 25 & 10052 \\
26 & 100 & 8362 \\
101 & 200 & 1656 \\
201 & 500 & 1073 \\
501 & 1000 & 407 \\
1001 & 2000 & 177 \\
$2001+$ & & 96 \\
\hline
\end{tabular}

(c) The number of shared edges betwen ASes, where \# AS pairs indicates how many pairs of ASes have at least Low and at most $U p$ shared edges.

Figure 12: The network characteristics of the internet graph used in the experiments for "Gain" and "Participation" 
selected pairs in seconds. As can be seen, the running time of Algorithm 2 quickly becomes very high.

\begin{tabular}{cccc}
\hline \# Requests & Algorithm I (s) & Algorithm 2 (s) & Ratio \\
\hline 1 & 0.02 & 0.09 & $1: 4.5$ \\
5 & 0.19 & 6.04 & $1: 31.79$ \\
10 & 1.09 & 84.31 & $1: 77.35$ \\
15 & 2.38 & 270.87 & $1: 113.81$ \\
19 & 4.01 & 519.27 & $1: 129.49$ \\
\hline
\end{tabular}

Table 1: The performance of Algorithm 1 compared to Algorithm 2.

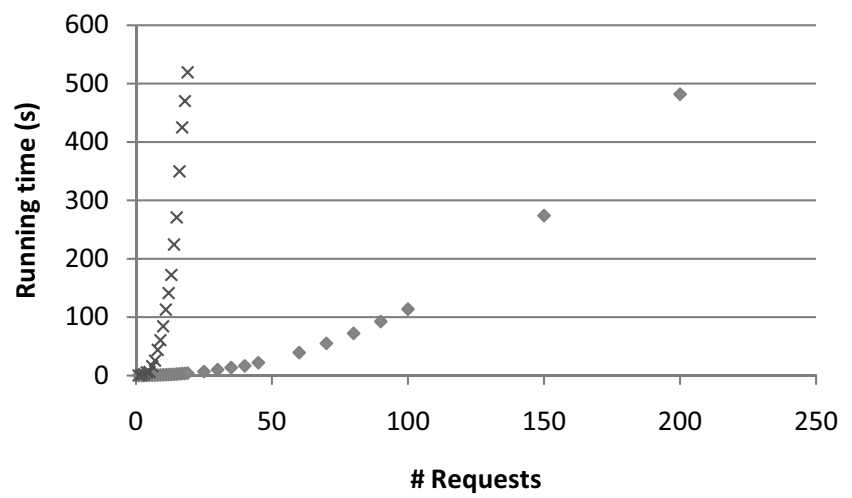

- Algorithm $1 \times$ Algorithm 2

Figure 13: Running times of both algorithms.

The memory usage is dominated by the number of Pareto optimal solutions, and each Pareto optimal solution is represented as a tuple of two integers. Figure 13 shows a graphical comparison of both algorithms. Not only is Algorithm I fast for small amounts of requests, it can handle up to ten times more requests in the same time as Algorithm 2. 


\section{Participation and Gain}

From Figure 14 we see that roughly $90 \%$ of all AS pairs profit from trading paths. While there are many assumptions underlying these experiments that are not neccesarily realistic, this indicates that many ASes would benefit from path trading in practice. The gains are modest, typically in the range of $0-10 \%$ and a small percentage has significantly higher gains i.e. more than $15 \%$. Even when the number of possible ways to route to another AS is limited, the gain in the same range.

The running times are, again, very fast and the real bottleneck is the calculation of shortest paths. In all cases computation time is at most seconds discounting the shortest path computations.

\subsection{CONCLUSIONS}

Despite that PATH Trading is NP-hard, we developed an algorithm that is fast in practice, which we explained theoretically by smoothed analysis. Our algorithm is robust, and gives great flexibility because it returns the whole set of Pareto-optimal path trades.

Throughout the paper we assumed that ASes are truthful and share their data. The real costs can, of course, be scaled to the interval $[0,1]$, where 1 are the costs of the hot-potato routing [79]. This would keep the real costs secret, but we would have a trade-off of relative gain and not absolute cost savings. Lying could potentially increase the cost savings of an AS, but no AS would be worse off than with hot-potato routing. Every AS simply refuses any path trade that costs more than their hot-potato strategy. Unfortunately, achieving a mechanism that is both truthful and efficient seems impossible [96].

The most promising direction for further research is to use real traffic data, and model this in an appropriate way to find out how large the real gains are for ASes. From a theoretical perspective, extending Corollary 7.4 from two ASes to a fixed number of ASes seems challenging. Even though there is a generalization of the results by Beier et al. [13] to multiobjective optimization problems with any constant number of objectives by Röglin and Teng [9o], this generalization cannot be applied directly to path trading with multiple ASes for some technical reasons. 


\begin{tabular}{rrr}
\hline Gain & \# AS pairs & $\%$ \\
\hline $0 \%$ & 2601 & $9.39 \%$ \\
$0-1 \%$ & 1321 & $4.77 \%$ \\
$1-2 \%$ & 2071 & $7.48 \%$ \\
$2-3 \%$ & 2373 & $8.56 \%$ \\
$3-4 \%$ & 2316 & $8.36 \%$ \\
$4-5 \%$ & 2418 & $8.73 \%$ \\
$5-6 \%$ & 2387 & $8.62 \%$ \\
$6-7 \%$ & 2186 & $7.89 \%$ \\
$7-8 \%$ & 1930 & $6.97 \%$ \\
$8-9 \%$ & 1664 & $6.01 \%$ \\
$9-10 \%$ & 1324 & $4.78 \%$ \\
$10-15 \%$ & 3617 & $13.06 \%$ \\
$15-20 \%$ & 981 & $3.54 \%$ \\
$20 \%+$ & 512 & $1.89 \%$ \\
\hline
\end{tabular}

(a) The number and percentage of AS pairs that had a certain amount of gain due to path trades. \# AS pairs and $\%$ are the number of AS pairs and the percentage of AS pairs, respectively that reduced costs by Gain.

\begin{tabular}{r|rrrr}
\hline & \multicolumn{4}{|c}{ Shared border size } \\
& $\mathbf{2}$ & $\mathbf{2 6}$ & $\mathbf{1 0 1}$ & $\mathbf{2 0 1 +}$ \\
Gain & $\mathbf{2 5}$ & $\mathbf{1 0 0}$ & $\mathbf{2 0 0}$ & \\
\hline $0 \%$ & 2126 & 390 & 32 & 53 \\
$0-1 \%$ & 3111 & 624 & 74 & 113 \\
$1-2 \%$ & 1526 & 403 & 68 & 80 \\
$2-3 \%$ & 1667 & 529 & 88 & 92 \\
$3-4 \%$ & 1537 & 611 & 97 & 86 \\
$4-5 \%$ & 1559 & 671 & 122 & 86 \\
$5-6 \%$ & 1424 & 680 & 133 & 157 \\
$6-7 \%$ & 1181 & 684 & 153 & 168 \\
$7-8 \%$ & 1048 & 595 & 155 & 150 \\
$8-9 \%$ & 890 & 517 & 114 & 147 \\
$9-10 \%$ & 696 & 449 & 82 & 136 \\
$10-15 \%$ & 1823 & 1181 & 285 & 334 \\
$15-20 \%$ & 467 & 362 & 92 & 94 \\
$20 \%+$ & 297 & 155 & 35 & 25 \\
\hline
\end{tabular}

(b) The number of AS pairs that had a certain amount of gain due to path trades split by the number of shared edges. An entry of the table is the number of AS pairs that reduced costs by Gain and that shared Shared border size edges.

Figure 14: The gains that ASes obtained by trading paths. 



\section{HOW TO CUT A GRAPH}

This chapter is on the following paper:

A. Berger, A. Grigoriev and R. van der Zwaan, "How to Cut a Graph into Many Pieces", in Theory and Applications of Models of Computation (TAMC) 2011. Lecture Notes in Computer Science 6648:184-194, 2011. [14].

\subsection{INTRODUCTION}

In this chapter we address the following graph separation problem, called $k$-WAy Vertex CUt : Given an n-vertex graph $G=(V, E)$ and integers $k \leqslant n$ and $s \leqslant n$, find a cut-set $S \subseteq \mathrm{V}$ of size at most $\mathrm{s}$ such that the subgraph of $G$ induced by $V \backslash S$ has at least $k$ components. Throughout the chapter we refer to this problem as k-WAy VerTex Cut. We remark that obtaining at least $k$ components while minimizing the number of cut-vertices, is NPhard to approximate within any factor. This is because any set of vertices separating the graph into at least $k$ components identifies an independent set of size at least $k$ [44]. Therefore, this chapter focuses on approximating the maximum number of components by removing at most $s$ vertices.

There are many natural graph-theoretic problems where one has to find a graph separator of a given size satisfying certain properties. This type of problems is very generic and has attracted the attention of many researchers. Moreover, graph separation problems have many applications, e.g., in routing and network reliability. There are many applications where the underlying network is (almost) planar, for example road networks. Therefore, investigating k-WAY VERTEX Cut restricted to planar graphs is interesting, especially when the problem turns out to be very difficult to solve, even approximately, on general graphs.

Surprisingly enough, despite its very basic and quite generic setting, $k$ WAy Vertex CUt did not receive much attention in the literature. Marx [81] has investigated the fixed-parameter (in)tractability of k-WAY Vertex Cut among 
other related problems. This is in contrast to the amount of research concerning closely related problems. Most importantly k-CUT, which we call k-Way Edge Cut, which is the "edge-version" of k-Way Vertex Cut. Given a graph $G=(V, E)$ and integers $k$ and $s$, remove at most $s$ edges so as to split the graph into at least $k$ components. We show that k-WAY VerTex Cut is a generalization of k-WAy Edge Cut.

Goldschmidt and Hochbaum prove the problem is NP-hard when $k$ is part of the input, but there is a polynomial time algorithm for fixed $k$ [49]. Later the running time was improved to $\mathrm{O}\left(\mathrm{n}^{(2-\mathrm{o}(1)) \mathrm{k}}\right)$ by Karger and Stein [67], but the algorithm is randomized. Kamidori, Yoshida and Nagamochi [66] present a deterministic $\mathrm{O}\left(\mathrm{n}^{(4+\mathrm{o}(1)) \mathrm{k}}\right)$ time algorithm which was improved by Thorup to $\tilde{\mathrm{O}}\left(\mathrm{mn}^{2 \mathrm{k}-2}\right)$ time [102].

Saran and Vazirani give a polynomial time approximation algorithm that finds at most $2 s$ edges that splits the graph into at least $k$ components [91]. For dense graphs, graphs with $\Omega\left(n^{2}\right)$ edges, Arora, Karger and Karpinski give a polynomial time approximation scheme [4]. Recently, Kawarabayashi and Thorup show that the problem is fixed parameter tractable in s [68], whereas parameterized by $k$ the problem is $W[1]$-hard [35].

There are several papers that studied k-WAY EDGE CUT on planar graphs. Hochbaum and Shmoys give a quadratic time algorithm for planar graphs when $k=3$ [6o]. He gave an improved algorithm for this special case with running time $\mathrm{O}(n \log n)$ [59]. Kawarabayashi and Thorup give an algorithm for graphs of bounded genus $\mathrm{g}$ with running time $\mathcal{O}\left(2^{\mathrm{O}\left(\mathrm{s}^{2} \mathrm{~g}^{2}\right)}\right)$ [68].

Guttman-Beck and Hasin give a polynomial time 3-approximation for a generalization and fixed $k$, where there are also restrictions on the number of vertices in components [55]. Related to k-WAy Vertex Cut is the notion of Bounded Fragmentation, introduced in [56]. A graph $G=(V, E)$ has bounded fragmentation if after removing any $\mathrm{S} \subseteq \mathrm{V}$ of size at most $s$ the number of components is bounded only by a function of $k$.

Two related problems, Multiway Cut and Multicut impose restrictions on which vertices should be separated. In Multiway Cut $k$ terminal vertices have to be separated by removing s edges (or vertices) [29, 32, 33, 44, 107], and for Multicut one has to find $s$ edges (or vertices) that separates each of $k$ given terminal-pairs [30, 31, 45, 52]. 


\subsubsection{Our results}

In this chapter we present the picture of the computational complexity and the approximability of k-WAy Vertex Cut for several natural classes of graphs.

In Section 8.2 we give a summary of complexity results for k-WAY VerTex CUT, using standard reductions from INDEPENDENT SET and a reduction for split graphs already used by Marx [81]. Also, we show that k-WAY VerTEx Cut can be solved in polynomial time for interval graphs and for graphs of bounded treewidth. We start this section, however, with an approximation preserving reduction from k-WAy EDGE Cut.

Section 8.4 contains our main contribution for this chapter, namely, an efficient polynomial-time approximation scheme (EPTAS) for k-WAY VERTEx CUT on planar graphs. The running time of our algorithm is $O\left(n k^{2}\left(\frac{1}{\varepsilon^{2}}\right)^{\mathrm{O}\left(\frac{1}{\varepsilon^{2}}\right)}\right)$, where $k$ is the upper bound on the size of the cut-set. We also present a fixed-parameter tractable (FPT) algorithm for planar graphs with running time

$\mathrm{O}\left(n \mathrm{k}^{\mathrm{O}(\mathrm{k})}\right)$ in the same section. The FPT algorithm is needed for the EPTAS.

\subsubsection{Notions and definitions}

The set of components in $\mathrm{G}$ is represented by $\mathrm{C}(\mathrm{G})$, where each element in $\mathcal{C}(G)$, a set of vertices, induces a connected graph. Let $c(G, S)$ denote $\mid \mathcal{C}(G \backslash$ $S)|-| \mathcal{C}(G) \mid$ i.e. the number of extra components obtained by removing $S$ from $\mathrm{G}$. Moreover, let the component number $\mathrm{k}(\mathrm{G}, \mathrm{s})$ of $\mathrm{G}$ be the maximum $c(G, S)$ over all subsets $S \subseteq V$ of size at most $s$.

\subsection{HARDNESS RESULTS}

In this section we prove three hardness results for k-Way Vertex Cut. First we show that k-WAy Vertex Cut generalizes k-Way Edge Cut.

Theorem 8.1. k-Way Vertex Cut generalizes k-Way Edge Cut.

Proof. Consider a graph $\mathrm{G}=(\mathrm{V}, \mathrm{E})$ with parameters $k$ and $s$ of $k$-WAY EDGE Cut we construct a graph $\mathrm{G}^{\prime}=(\mathrm{W}, \mathrm{F})$ as follows. In $W$ there is a vertex $\mathcal{W}_{v}$ 
for every vertex $v \in V$ and a vertex $w_{u, v}$ for every edge $\{u, v\} \in E$. Create the edge set $F$ as follows, if $\{u, v\} \in E$ then $\left\{w_{u}, w_{u, v}\right\} \in F$ and $\left\{w_{u, v}, w_{v}\right\} \in F$. Further, for all $\{x, y\},\{x, z\} \in E$ we have $\left\{w_{x, y}, w_{x, z}\right\} \in F$.

Let $C \subseteq E$ a subset of at most $s$ edges in $\mathrm{G}$ such that removing $\mathrm{C}$ splits $\mathrm{G}$ into at least $k$ components. Then, then the cut-set $S:=\left\{w_{u, v} \in F \mid\{u, v\} \in\right.$ $C$ \} in $G^{\prime}$ consists of at most $s$ vertices that splits the graph into at least $k$ components.

Let $S \subseteq W$ be a cut-set of at most $s$ vertices in $G^{\prime}$ such that $G^{\prime}[W \backslash S]$ consists of at least $\mathrm{k}$ components. Suppose that for any $v \in \mathrm{V}$ we have $w_{v} \in S$. We claim that we can remove $w_{v}$ from $S$ and that removing $S$ splits the graph into at least $k$ components. If $w_{v}$ has at most one neighbour that is not in the cut-set, the claim is trivially true. Note that there is no $u \in V$ such that $\left\{w_{u}, w_{v}\right\} \in F$. Therefore, suppose $w_{v}$ has two neighbours $w_{v, x}$ and $w_{v, y}$ that are in different components in $\mathrm{G}^{\prime}$ after removing $\mathrm{S}$. However, by construction $\left\{w_{v, x}, w_{v, y}\right\} \in F$, a contradiction. Thus, we can assume that the cut-set $\mathrm{S}$ in $\mathrm{G}^{\prime}$ consists only of vertices that correspond to edges in $\mathrm{G}$. This immediately yields the theorem.

Secondly, we show that k-WAy Vertex Cut is a generalization of the INDEPENDENT SET problem, which yields the following theorem.

Theorem 8.2. It is NP-hard to approximate k-WAY VERTEx Cut within a factor of $n^{1-\varepsilon}$ for every $\varepsilon>0$. Moreover, $k$-WAy Vertex Cut is NP-hard on 3-regular planar graphs.

Proof. In order to prove both statements we will give an approximation preserving reduction from INDEPENDENT SET (IS). It is NP-hard to approximate the INDEPENDENT SET problem within a factor of $n^{1-\varepsilon}$ for every $\varepsilon>0$ [109]. Moreover, the INDEPENDENT SET problem is NP-complete on 3-regular planar graphs [42].

Given a graph $G=(V, E)$, the IndePENDENT SET problem asks for a subset $S \subseteq \mathrm{V}$ of maximum cardinality such that $\mathrm{G}[\mathrm{S}]=\emptyset$. We consider the k-Way Vertex Cut problem when restricted to the case $s=n$. In this case, any independent set $\mathrm{I} \subseteq \mathrm{V}$ of size $\mathrm{k}$ gives rise to a cut-set $\mathrm{S}=\mathrm{V} \backslash \mathrm{I}$ of size $n-k$ with $c(G, S)=k-|\mathcal{C}(G)|$, since each vertex in I must lie in a different component of $G \backslash S$ (remember $c(G, S)$ is the number of additional components when removing $S$ ). Conversely, any cut-set $S \subseteq \mathrm{V}$ gives rise to an independent set of size $c(G, S)+|\mathcal{C}(G)|$ by picking one vertex from each component in $\mathrm{G}[\mathrm{V} \backslash \mathrm{S}]$. Hence we have that $\mathrm{k}(\mathrm{G}, \mathrm{n})=\mathrm{OPT}_{\mathrm{IS}}-|\mathcal{C}(\mathrm{G})|$. 
Moreover, any $\alpha$-approximate $(0 \leqslant \alpha \leqslant 1)$ solution $S \subseteq \mathrm{V}$ to the k-WAY Vertex Cut problem with $c(G, S) \geqslant \alpha \kappa(G, n)$ gives rise to an independent set of size at least $c(G, S)+|\mathcal{C}(\mathrm{G})| \geqslant \alpha\left(\mathrm{OPT}_{\mathrm{IS}}-|\mathcal{C}(\mathrm{G})|\right)+|\mathcal{C}(\mathrm{G})| \geqslant \alpha \mathrm{OPT}_{\mathrm{IS}}$, which completes the proof.

A graph $G=(V, E)$ is a split graph if and only if there is a partition $V=A \cup B$ of the vertices such that $A$ forms an independent set and $B$ forms a clique. Marx [81] has shown that k-WAY Vertex CUt is W[1]-hard with parameter $k$ on split graphs by using a reduction from MAXIMUM Clique. We use a similar reduction to reduce k-Way Vertex Cut to Dense k-SUBGRAPH in an approximation preserving way. Together with an inapproximability result by Khot [70], this yields the following theorem.

Theorem 8.3. k-WAy VeRTex Cut is NP-complete and W[1]-hard when restricted to split graphs. Moreover, it does not admit a PTAS for split graphs unless $\mathrm{NP} \subseteq \cap_{\varepsilon}>0$ BPTIME $\left(2^{\mathfrak{n}^{\varepsilon}}\right)$.

Proof. We give an approximation preserving reduction from DeNSE k-SUBGRAPH. Given a graph $G=(V, E)$ the DENSE k-SubGRAPH problem asks for a subset $\mathrm{S} \subseteq \mathrm{V}$ of cardinality at most $\mathrm{k}$ such that the number of edges in $\mathrm{G}[\mathrm{S}]$ is maximized. From a given instance of DeNSE k-SubgraPH we construct a split graph $G^{\prime}=\left(A \cup B, E^{\prime}\right)$ as follows. First we let $B=V$ and all vertices in $B$ form a clique. Moreover, we let $A=E$, i.e. each vertex in $A$ corresponds to an edge in $G$. For every edge $e=u v \in E$ we add the edges $e u$ and $e v$ to $E^{\prime}$. We now claim that the optimal values for Dense k-SubGraph in $G$ $\left(\mathrm{OPT}_{\mathrm{DKS}}\right)$ and $k$-WAY VerTex Cut with parameter $s=k$ in $\mathrm{G}^{\prime}\left(\mathrm{k}\left(\mathrm{G}^{\prime}, \mathrm{s}\right)\right)$ are equal.

Suppose that there is a set $S \subseteq \mathrm{V}$ of size $\mathrm{k}$ that induces a subgraph in $\mathrm{G}$ with $\mathrm{m}$ edges. By taking $\mathrm{S}$ as cut in $\mathrm{G}^{\prime}$ we get $\mathrm{m}$ components (isolated vertices in $A)$, i.e. $k\left(G^{\prime}, s\right) \geqslant c\left(G^{\prime}, S\right)=\mathrm{OPT}_{\mathrm{Dks}}$.

Now let $S \subseteq A \cup B$ be an optimal cut-set of size at most $s=k$ such that $c\left(G^{\prime}, S\right)=\kappa\left(G^{\prime}, s\right)$. We can assume that $S$ only contains clique vertices from $B$, since otherwise we can remove vertices in $A$ from $S$ while not decreasing $c\left(G^{\prime}, S\right.$. Therefore, $S \subseteq V$ and $G^{\prime}[V \backslash S]$ contains only vertices corresponding edges from $\mathrm{G}[\mathrm{S}]$ plus one extra component. Thus $\mathrm{OPT}_{\mathrm{DkS}} \geqslant$ $c\left(G^{\prime}, S\right)=\kappa\left(G^{\prime}, s\right)$ and the claim follows. 


\subsection{POLYNOMIAL CASES}

In this section we show that k-WAY VerTex Cut can be solved optimally for interval graphs and graphs of bounded treewidth. It is known, see Booth and Lueker [19], that any interval graph can be decomposed into a sequence of maximal cliques. The following lemma precisely describes this decomposition.

Lemma 8.4 (Booth and Lueker, [19]). Let $\mathrm{G}=(\mathrm{V}, \mathrm{E})$ be an interval graph on $\mathrm{n}$ vertices and $\mathrm{m}$ edges. Then, one can find in $\mathrm{O}(\mathrm{n}+\mathrm{m})$ time a sequence of vertex sets $\mathrm{X}_{1}, \ldots, \mathrm{X}_{\mathrm{t}}$ such that

- $X_{j}, 1 \leqslant j \leqslant t$, is a maximal clique;

- if $v \in X_{\mathfrak{j}^{\prime}}$ and $v \in X_{\mathbf{j}^{\prime \prime}}$ then $v \in \mathbf{X}_{\mathbf{j}}$ for all $\mathbf{j}^{\prime}<\mathbf{j}<\mathbf{j}^{\prime \prime}$;

- for all $e \in \mathrm{E}$, there is $\mathrm{j} \in\{1, \ldots, \mathrm{t}\}$ such that $e \in \mathrm{G}\left[\mathrm{X}_{\mathrm{j}}\right]$.

Further we refer to the vertex sets $X_{j}, 1 \leqslant j \leqslant t$, as bags.

Theorem 8.5. Let $\mathrm{G}=(\mathrm{V}, \mathrm{E})$ be an interval graph and let $\mathrm{k}$ be an integer. Then, $\mathrm{k}(\mathrm{G}, \mathrm{k})$ can be found in $\mathrm{O}\left(\mathrm{n}^{2} \mathrm{k}\right)$ time.

Proof. Using the structure of interval graphs from Lemma 8.4, we design a dynamic programming algorithm solving k-WAY VERTEX CUT in $O\left(n^{2} k\right)$ time. In fact, Lemma 8.4 provides a path decomposition of graph G.

First we show that there is an optimal cut-set that consists of the union of several minimal separators. Let $S$ be the optimal cut-set in $\mathrm{G}$ of cardinality at most $s$ that splits the graph into at least $k$ components, we will modify $S$ such that it is a union of minimal separators of cardinality at most $s$ that splits the graph into at least $k$ components. Let $j$ the the smallest integer such that $X_{j} \cap X_{j+1} \subseteq S$, in other words the leftmost minimal separator that is contained in $S$. Let $L$ be the set of vertices in bags 1 to $j$ but not in any bag beyond j e.g. $L:=\cup_{1 \leqslant i \leqslant j} X_{i} \backslash X_{j+1}$. Because of the minimality of $j$ we have that $S \cap L$ is not a separator in $G[L]$ and $G[L \backslash S]$ is a single component. Therefore, if $S$ splits the graph into at least $k$ components, so does $S \backslash L$. Let $S:=S \backslash L$ and apply the same argument on the graph $G\left[V \backslash L \backslash\left(X_{j} \cap X_{j+1}\right)\right]$. Therefore we can conclude that there is an optimal cut-set that is a union of minimal separators.

In interval graphs every minimal separator is the intersection of two consecutive bags. Therefore, we can restrict our attention to cut-sets formed 
by the unions of the intersections of the (pairs of) consecutive bags. In the dynamic program we move along the sequence (say, along the path decomposition from left to right) recursively selecting the right-most intersections of the consecutive bags for the cut-set and keeping track of the cardinality of the cut-set. This is a standard Bellman-Ford type of dynamic programming running in $\mathrm{O}\left(\mathrm{n}^{2} \mathrm{k}\right)$ time and requiring only $\mathrm{O}(\mathrm{nk})$ space.

We now present a dynamic programming algorithm for k-WAY VerTex Cut on graphs of bounded treewidth. We assume that a tree decomposition is given as part of the input, there are several algorithms for finding the tree decomposition of a graph, for example see Bodlaender [17].

Definition 8.6. $A$ tree-decomposition of $\mathrm{G}$ is a pair $\left(\left\{\mathrm{X}_{\mathrm{i}}: \mathrm{i} \in \mathrm{I}\right\}, \mathrm{T}=(\mathrm{I}, \mathrm{F})\right)$ with $\left\{X_{i}: i \in I\right\}$ being a family of subsets of $\mathrm{V}$, one for each node of $\mathrm{T}$, and $\mathrm{T}$ a tree such that

1. $U\left(X_{i}: i \in I\right)=V$.

2. Every edge of $\mathrm{G}$ has both its ends in some $\mathrm{X}_{i}(\mathrm{i} \in \mathrm{I})$.

3. For $i, j, l \in I$, if $l$ lies on the path from $i$ to $j$ in $T$ then $X_{i} \cap X_{j} \subseteq X_{l}$.

The width of the tree-decomposition is $\max \left\{\left|X_{i}\right|-1: i \in I\right\}$. The treewidth of $\mathrm{G}$ is the minimum width of all tree-decompositions of $\mathrm{G}$. Conventionally, we call a each set $X_{i}(i \in I)$ a bag of the tree decomposition.

A tree decomposition $(X, T)$ of width $w$ can be transformed in linear time to a nice tree decomposition of equal width [74].

Definition 8.7. A nice tree decomposition of $\mathrm{G}$ is a tree decomposition $(\mathrm{X}, \mathrm{T})$ of $\mathrm{G}$, where in addition $\mathrm{T}$ is a directed tree rooted at some $\mathrm{r} \in \mathrm{I}$, such that

1. every node in $\mathrm{T}$ has at most two children,

2. if a node $i$ in $T$ has two children $j_{1}$ and $j_{2}$, then $X_{i}=X_{j_{1}}=X_{j_{2}}$ ( $i$ is a join node);

3. if a node $i$ has one child $j$, then either

- $\left|X_{i}\right|=\left|X_{j}\right|+1$ and $X_{j} \subset X_{i}$ (i is an introduce node);

- $\left|X_{i}\right|=\left|X_{j}\right|-1$ and $X_{i} \subset X_{j}$ (i is a forget node). 
Theorem 8.8. Let $\mathrm{G}=(\mathrm{V}, \mathrm{E})$ be a graph with treewidth at most $w$ and let $\mathrm{s} \leqslant|\mathrm{V}|$ be an integer. Then, $\mathrm{k}\left(\mathrm{G}, \mathrm{s}^{\prime}\right)$ can be found in $\mathrm{O}\left(\mathrm{ns}^{2}(w+1)^{w}\right)$ time for all $0 \leqslant s^{\prime} \leqslant s$.

Proof. Let $\left(\left\{X_{i}: i \in I\right\}, T=(I, F)\right)$ be a tree decomposition of $G$ of width $w$ rooted at $r \in I$. Let $T_{i}$ be the set of vertices in the subtree of $T$ rooted at $i$ and let $Q_{i}=\bigcup_{j \in T_{i}} X_{j}$.

Now let $i$ be a node in $I, C \subseteq X_{i}, \mathcal{P} \in w^{X_{i} \backslash C}$ and $s^{\prime} \leqslant s$. Then we define $m_{i}\left(C, \mathcal{P}, s^{\prime}\right)$ to be the maximum number of additional components in $G\left[Q_{i} \backslash X_{i}\right]$ that can be obtained by removing a set $S \subset Q_{i} \backslash X_{i}$ of size at most $s^{\prime}$, such that two vertices in $X_{i} \backslash C$ are in the same component when $S \cup \mathrm{C}$ is removed if and only if they are in the same set in the partition $\mathcal{P}$. Informally, we try all different ways to partition a bag into a cut-set and components. The property that we want to maintain is that if vertices are assigned to different partitions in a bag, then either they are not connected in $G\left[Q_{i}\right]$ or the value of this partition is $-\infty$. For simplicity partitions are not allowed in which two vertices $u$ and $v$ are in different sets, but $u v \in E$. Now we can compute the values $m_{i}\left(C, \mathcal{P}, s^{\prime}\right)$ for the different types of nodes as follows.

LEAF NOdes. Let $i$ be a leaf node. Then $m_{i}\left(C, \mathcal{P}, s^{\prime}\right)=0$, since $Q_{i} \backslash X_{i}=\emptyset$ in this case.

JOIN NODES. Let $i$ be a join-node with children $j_{1}$ and $j_{2}$. Then we have that

$$
m_{i}(C, \mathcal{P}, k)=\max _{s_{1}+s_{2}=s^{\prime}} m_{l}\left(C, \mathcal{P}, s_{1}\right)+m_{r}\left(C, \mathcal{P}, s_{2}\right) .
$$

Since $X_{i}=X_{j_{1}}=X_{j_{2}}$ we use the same cut-set $C$ and partition $\mathcal{P}$. In $m_{i}\left(C, \mathcal{P}, s^{\prime}\right)$ we are required to obtain the maximum number of components using $s^{\prime}$ cut-vertices, and components in the two subtrees do not overlap, so we can split $s^{\prime}$ over the two children.

INTRODUCE NODES. Let $i$ be an introduce node with introduced vertex $v$ and child j. If $v \in \mathrm{P} \in \mathcal{P}$ then we let $\mathcal{P}-v:=\mathcal{P} \backslash\{\mathrm{P}\} \cup\{\mathrm{P} \backslash\{v\}\}$. Then we have that

$$
m_{\mathfrak{i}}\left(C, \mathcal{P}, s^{\prime}\right)= \begin{cases}m_{\mathfrak{j}}\left(C \backslash\{v\}, \mathcal{P}, s^{\prime}\right) & \text { if } v \in C \\ m_{\mathfrak{j}}\left(C, \mathcal{P}-v, s^{\prime}\right) & \text { otherwise. }\end{cases}
$$

The correctness follows easily. Type explanation. 
FORGET NODES. Let $i$ be a forget node with forgotten vertex $v$ and child $j$. Then we have that

$$
m_{i}\left(C, \mathcal{P}, s^{\prime}\right)=\max \left\{m_{i}^{\text {out }}\left(C, \mathcal{P}, s^{\prime}\right), m_{i}^{\text {in }}\left(C, \mathcal{P}, s^{\prime}\right)\right\}
$$

where $m_{i}^{\text {out }}$ is the case where $v$ is not in the cut-set and $m_{i}^{i n}$ where $v$ is in the cut-set:

$$
m_{i}^{\text {out }}\left(C, \mathcal{P}, s^{\prime}\right)= \begin{cases}m_{j}\left(C, \mathcal{P} \cup\{v\}, s^{\prime}\right)+1 & \text { if } N(v) \cap X_{i} \subseteq C \\ \max _{P \in \mathcal{P}} m_{j}\left(C,(\mathcal{P} \backslash\{P\}) \cup\{P \cup\{v\}\}, s^{\prime}\right) & \text { otherwise, }\end{cases}
$$

and

$$
m_{i}^{i n}\left(C, \mathcal{P}, s^{\prime}\right)=m_{j}\left(C \cup\{v\}, \mathcal{P}, s^{\prime}-1\right)
$$

If $v$ is in the optimal cut-set we lower $s^{\prime}$ by one and we take the value of $m_{j}$ with cut-set $C \cup\{v\}$. If $v$ is not in the cut-set then we have to prevent $v$ from connecting several partitions in $X_{i}$ : this would invalidate such a partition. If all neighbors of $v$ are in the cut-set, then $v$ is part of a component that is now completely disconnected from higher up the tree, and we can increase $m_{i}\left(C, \mathcal{P}, s^{\prime}\right)$ by one. Otherwise, if $v$ is a neighbor to one partition $P_{i}$ we add $v$ to that partition, if $v$ is a neighbor to more than one partition, then the value is not valid and the value is $-\infty$.

ROOT NODE. Let $r$ be the root node of the tree decomposition. Then the optimal value $\kappa(G, s)$ can be computed as follows.

$$
\kappa(G, s)=\max _{\substack{c \subseteq X_{r},|C|+s^{\prime} \leqslant s \\ \mathcal{P} \in w^{\prime} X_{i} \backslash C}} m_{r}\left(C, \mathcal{P}, s^{\prime}\right)+|\mathcal{P}|
$$

We solve these recurrences by dynamic programming. There are at most $(w+1)^{w}$ partitions of a bag into $C$ and $\mathcal{P}$, and we cut at most $s$ vertices. Therefore there are at most $s(w+1)^{w}$ different values for any $m_{i}(\cdot, \cdot, \cdot)$. The computation for each $m_{i}(C, \mathcal{P}, s)$ takes at $\operatorname{most} \mathrm{O}(\max \{w, s\})$ time and there are at most $\mathrm{O}(n)$ bags in the tree decomposition. We can find $\kappa\left(G, s^{\prime}\right)$ for all $s^{\prime} \leqslant s$, given a tree decomposition $\left(\left\{X_{i}: i \in I\right\}, T=(I, F)\right)$ of $G$ in $\mathrm{O}\left(n s^{2}(w+1)^{w}\right)$ time. 


\subsection{FIXED-PARAMETER TRACTABILITY AND AN EP- TAS FOR PLANAR GRAPHS}

In this section we develop algorithms for the k-WAY VeRTEX CUT problem on planar graphs. We assume that a planar embedding is given as a part of the input, otherwise we construct it in linear time using Hopcroft and Tarjan's algorithm [61]. An embedding of a graph G is called outerplanar (or 1 -outerplanar) if all vertices of the graph are incident to the outer face. For a positive integer $p \geqslant 2$, an embedding of $G$ is called $p$-outerplanar if the removal of all vertices incident to the outer face yields a $(p-1)$-outerplanar graph. Assume without loss of generality, that every component in G is incident to the outer face in the embedding. We denote the vertices incident to the outer face by layer $\mathrm{L}_{1}$. Recursively, all vertices incident to the outer face of the graph obtained by removing layers $L_{1}$ to $L_{j-1}$ form layer $L_{j}$ $(j \geqslant 2)$. The set of layers of a plane graph $G$ is denoted as $\mathcal{L}:=\left\{L_{1}, \ldots, L_{z}\right\}$. Given a plane graph $G, \mathcal{L}$ can be found in linear time by a slightly modified BFS-algorithm where the search procedure is done in face-by-face fashion. From now on, we assume that in all algorithms, $\mathcal{L}$ is given as a part of the input.

We will first show that k-Way Vertex Cut on planar graphs is fixedparameter tractable with respect to parameter $k$. We will make use of the following lemma.

Lemma 8.9 (Bodlaender, [18]). Let $\mathrm{G}=(\mathrm{V}, \mathrm{E})$ be a p-outerplanar graph on $\mathrm{n}$ vertices. Then, a tree decomposition of width at most $3 p-1$ can be found in $\mathrm{O}(\mathrm{n})$ time.

We will first investigate the structure of the optimal solution in case a given family of layers is forbidden in the cut-set. Let $\mathcal{F}:=\left\{\mathrm{L}_{i_{1}}, \ldots, \mathrm{L}_{\mathrm{i}_{\mathrm{r}}}\right\} \subset \mathcal{L}$ be a set of forbidden layers, i.e., for any $v \in \mathrm{L}_{i_{\mathrm{t}}}, 1 \leqslant \mathrm{t} \leqslant \mathrm{r}$, vertex $v$ cannot be present in the cut-set. Without loss of generality we assume that $1 \leqslant$ $i_{1}<\cdots<i_{r} \leqslant z$. We denote by $\kappa(G, \mathcal{F}, s)$ the maximum of $c(G, S)$ over all $S \subset V$ such that $S \cap \bigcup_{t=1}^{r} L_{i_{t}}=\emptyset$ and $|S| \leqslant s$. Additionally, we set $i_{0}:=1$ and $i_{r+1}:=z$. For $0<t<r$, let $P_{t}:=\bigcup_{l=i_{r-t}}^{i_{r-t+1}} L_{l}$, and $P_{0}:=\bigcup_{l=i_{r}}^{z} L_{l}$ and $P_{r}:=\bigcup_{l=1}^{i_{1}} L_{l}$. 
Lemma 8.10. Let $\mathrm{G}=(\mathrm{V}, \mathrm{E})$ be a plane graph, let $\mathrm{s} \geqslant 1$ be an integer and let $\mathcal{F}$ be a set of forbidden layers as denoted above. Then, there exist non-negative integers $\mathrm{s}_{0}, \ldots, \mathrm{s}_{\mathrm{r}}$ such that the following holds:

$$
\begin{aligned}
& \text { - } k(G, \mathcal{F}, s)=\sum_{t=0}^{r} \kappa\left(G\left[P_{t}\right], \mathcal{F}, s_{t}\right), \\
& \text { - } \sum_{t=0}^{r} s_{t}=s .
\end{aligned}
$$

Proof. Consider an arbitrary cut-set $S \subseteq \mathrm{V} \backslash \bigcup_{t=0}^{r} L_{i_{t}}$. We use induction in the number $r$ of layers in $\mathcal{F}$ to prove that $c(G, S)=\sum_{t=0}^{r} c\left(G\left[P_{t}\right], S\right)$. Since $S$ is an arbitrary cut-set, the claim of the lemma would follow immediately. The base case with no forbidden layers holds trivially.

Let $P_{j}^{\prime}:=\bigcup_{t=r-j}^{r} P_{t}, 0 \leqslant j \leqslant r$. Suppose

$$
c\left(G\left[P_{j}^{\prime}\right], S\right)=\sum_{t=r-j}^{r} c\left(G\left[P_{t}\right], S\right)
$$

holds for some $j, 0 \leqslant j \leqslant r$, then we claim that

$$
c\left(G\left[P_{j+1}^{\prime}\right], S\right)=c\left(G\left[P_{j}^{\prime}\right], S\right)+c\left(G\left[P_{j+1}\right], S\right) .
$$

Let us remind that by definition $c(G, S)$ is the number of extra components in $G$ after removal of $S$. Since $G\left[P_{j}^{\prime}\right]$ and $G\left[P_{j+1}\right]$ are two intersecting subgraphs, $c\left(G\left[P_{j}^{\prime} \cup P_{j+1}\right], S\right) \leqslant c\left(G\left[P_{j}^{\prime}\right], S\right)+c\left(G\left[P_{j+1}\right], S\right)$. We denote the overcount of adding up $c\left(G\left[P_{j+1} \backslash S\right]\right)$ and $c\left(G\left[P_{j}^{\prime} \backslash S\right]\right)$, the total amount that each component is counted more than once summed over all components, by

$$
\Delta:=c\left(G\left[P_{j+1} \backslash S\right]\right)+c\left(G\left[P_{j}^{\prime} \backslash S\right]\right)-c\left(G\left[P_{j}^{\prime} \cup P_{j+1} \backslash S\right]\right) .
$$

Note that only components that include vertices from $L_{i_{j}}$ can be counted multiple times. 
Assume $\Delta=\mathrm{c}\left(\mathrm{G}\left[\mathrm{L}_{\mathrm{i}_{\mathrm{j}}}\right]\right)$. Then,

$$
\begin{aligned}
c & \left(G\left[P_{j+1}^{\prime}\right], S\right) \\
= & c\left(G\left[P_{j}^{\prime} \cup P_{j+1}\right], S\right) \\
= & c\left(G\left[P_{j+1} \cup P_{j}^{\prime} \backslash S\right]\right)-c\left(G\left[P_{j+1} \cup P_{j}^{\prime}\right]\right) \\
= & c\left(G\left[P_{j+1} \backslash S\right]\right)+c\left(G\left[P_{j}^{\prime} \backslash S\right]\right)-\Delta-c\left(G\left[P_{j+1} \cup P_{j}^{\prime}\right]\right) \\
= & c\left(G\left[P_{j+1} \backslash S\right]\right)+c\left(G\left[P_{j}^{\prime} \backslash S\right]\right)-c\left(G\left[L_{i j}\right]\right) \\
& -c\left(G\left[P_{j+1} \cup P_{j}^{\prime}\right]\right) \\
= & c\left(G\left[P_{j+1} \backslash S\right]\right)-c\left(G\left[P_{j+1}\right]\right)+c\left(G\left[P_{j}^{\prime} \backslash S\right]\right) \\
& -c\left(G\left[P_{j}^{\prime}\right]\right) \\
= & c\left(G\left[P_{j+1}\right], S\right)+c\left(G\left[P_{j}^{\prime}\right], S\right) .
\end{aligned}
$$

Here, Equation 45 is true by definition of the set $\mathrm{P}_{j+1}^{\prime}$, Equation 46 follows from the definition of $c(\cdot, \cdot)$, Equation 47 is by Equation 44 . By assumption that $\Delta=\mathrm{c}\left(\mathrm{G}\left[\mathrm{L}_{i_{j}}\right]\right)$, we derive Equation 48. By construction of layers in planar graphs, $c\left(G\left[P_{j}^{\prime}\right]\right)=c\left(G\left[L_{i_{j}}\right]\right)$ and $c\left(G\left[P_{j+1}\right]\right)=c\left(G\left[P_{j+1} \cup P_{j}^{\prime}\right]\right)$, which yields Equation 49. Finally, Equation 50 follows again from the definition of $\mathrm{c}(\cdot, \cdot)$.

Thus, to prove Equation 43 it only remains to verify the assumption $\Delta=$ $c\left(G\left[L_{i_{j}}\right]\right)$. Consider a component $C \in \mathcal{C}\left(G\left[\left(P_{j}^{\prime} \cup P_{j+1}\right) \backslash S\right]\right)$ that intersects $L_{i_{j}}$. Let $A:=G\left[P_{j+1} \backslash(S \cap C)\right]$. Now, we intend to prove that $A$ is connected. For a contradiction, suppose there are at least two components in $A$, say, $A^{\prime}$ and $A^{\prime \prime}$. By definition, $G[C]$ is connected in $G\left[P_{j}^{\prime} \cup P_{j+1}\right]$. However, $A^{\prime}$ and $A^{\prime \prime}$ are not connected in $G\left[P_{j+1}\right]$. Thus, there must be a path connecting $A^{\prime}$ and $A^{\prime \prime}$ through $G\left[P_{j}^{\prime} \cap C\right]$ and not $G\left[P_{j+1} \cap C\right]$. On the other hand, by construction of layers, every path from $A^{\prime}$ to $A^{\prime \prime}$ through $G\left[P_{j}^{\prime} \cap C\right]$ must go through $\mathrm{L}_{i_{j}}$, from which we can shortcut the paths, such that the modified path only goes through $\mathrm{G}\left[\mathrm{P}_{\mathrm{j}+1} \cap \mathrm{C}\right]$; a contradiction.

Component $C$ can be covered by one component from $G\left[\left(P_{j+1} \cap C\right) \backslash S\right]$ and several components from $G\left[\left(P_{j}^{\prime} \cap C\right) \backslash S\right]$. Then, the number of times $C$ is counted too often is precisely the number of components from $G\left[P_{j}^{\prime} \cap\right.$ $C \backslash S$ ] that cover $C$. Further, at most one component from $\mathcal{C}\left(P_{j}^{\prime} \backslash S\right)$ contains vertices from any component induced by the forbidden layer $L_{i_{j}}$. Therefore, $\Delta \leqslant c\left(G\left[L_{i_{j}}\right]\right)$. Moreover, from the fact that every component induced by $L_{i_{j}}$ is contained in some component from $\mathcal{C}\left(\mathrm{P}_{j}^{\prime} \backslash S\right)$, it follows that $\Delta \geqslant c\left(G\left[\mathrm{~L}_{i_{j}}\right]\right)$. Therefore, $\Delta=\mathrm{c}\left(\mathrm{G}\left[\mathrm{L}_{i_{j}}\right]\right)$. 
We now present an FPT algorithm for k-WAY VeRTEX CUT on planar graphs with respect to parameter s, the pseudo-code is given in Algorithm 8 . The main structural property that we use in the algorithm is that among any $s+1$ consecutive layers there must be one layer without any cut-vertices. Similar ideas are driving all Baker's type polynomial-time approximation schemes [7] for combinatorial problems on planar graphs. By Lemma 8.12 we can contract the graph into a graph of treewidth bounded by $\mathrm{O}(\mathrm{s})$, if we know the set of forbidden layers not containing any cutvertices.

Theorem 8.11. Let $\mathrm{G}=(\mathrm{V}, \mathrm{E})$ be a planar graph and let $\mathrm{k}$ be an integer. Then, Algorithm 8 finds $\mathrm{k}(\mathrm{G}, \mathrm{k})$ in $\mathrm{O}\left(\mathrm{nk}^{\mathrm{O}(\mathrm{k})}\right)$ time.

Proof. Given a planar graph $\mathrm{G}=(\mathrm{V}, \mathrm{E})$ and integer $k$, let $\mathrm{G}[i, j]$ be the graph induced by $\bigcup_{i \leqslant t \leqslant j} L_{t}$. Algorithm 8 consists of two parts. First, we decompose the graph into smaller subgraphs of bounded treewidth. On these subgraphs we run the algorithm from Theorem 8.8. Using Lemma 8.10, in the second phase we apply dynamic programming to combine the obtained solutions on the subgraphs, and to return the overall optimal solution. Correctness follows from the following inductive argument. Assume we know $k\left(G[1, i], k^{\prime}\right)$ for some $i$ and $k^{\prime}$, then one of the layers $L_{i+1}, \ldots, L_{i+k+1}$ does not contain cut-vertices. Therefore, we can extend the solution for $G[1, i]$ to a solutions for $G[1, j]$ where $j \in[i+1, i+k+1]$. We can combine these solutions by Lemma 8.10.

The first part (lines 2 to 7 in Algorithm 8) takes $\mathrm{O}\left(\mathrm{nk}^{3} \mathrm{k}^{\mathrm{O}(\mathrm{k})}\right)$ time to calculate $k\left(G[q, q+d+1], \mathcal{F}, k^{\prime}\right)$, as in Algorithm 8. The union of all these small pieces contains at most $2 n$ vertices, and the treewidth of each $G[q, q+d+1]$ is bounded by $3(k+2)-1$ and can be found in linear time by Lemma 8.9. Therefore, by applying the algorithm in Theorem 8.8 we obtain the optimal solution in each small piece in $\mathrm{O}\left(\mathrm{nk}^{\mathrm{O}(\mathrm{k})}\right)$ time. The second part (lines 9 to 13) combines all parts by dynamic programming. This takes $\mathrm{O}\left(n k^{2}\right)$ time. The total running time is $\mathrm{O}\left(\mathrm{nk}^{\mathrm{O}(\mathrm{k})}\right)$.

We now move to the presentation of the efficient polynomial-time approximation scheme (EPTAS) for k-WAY VeRTEX CUT on planar graphs. Let us remind that an EPTAS (for a maximization problem) is a polynomialtime approximation algorithm that for any given $\varepsilon>0$ returns a solution of value at least $(1-\varepsilon)$ times the optimum in time poly $(n) f(\varepsilon)$, where poly $(n)$ only depends on $n$ and not on $\varepsilon$, and $f(\cdot)$ is some function only 


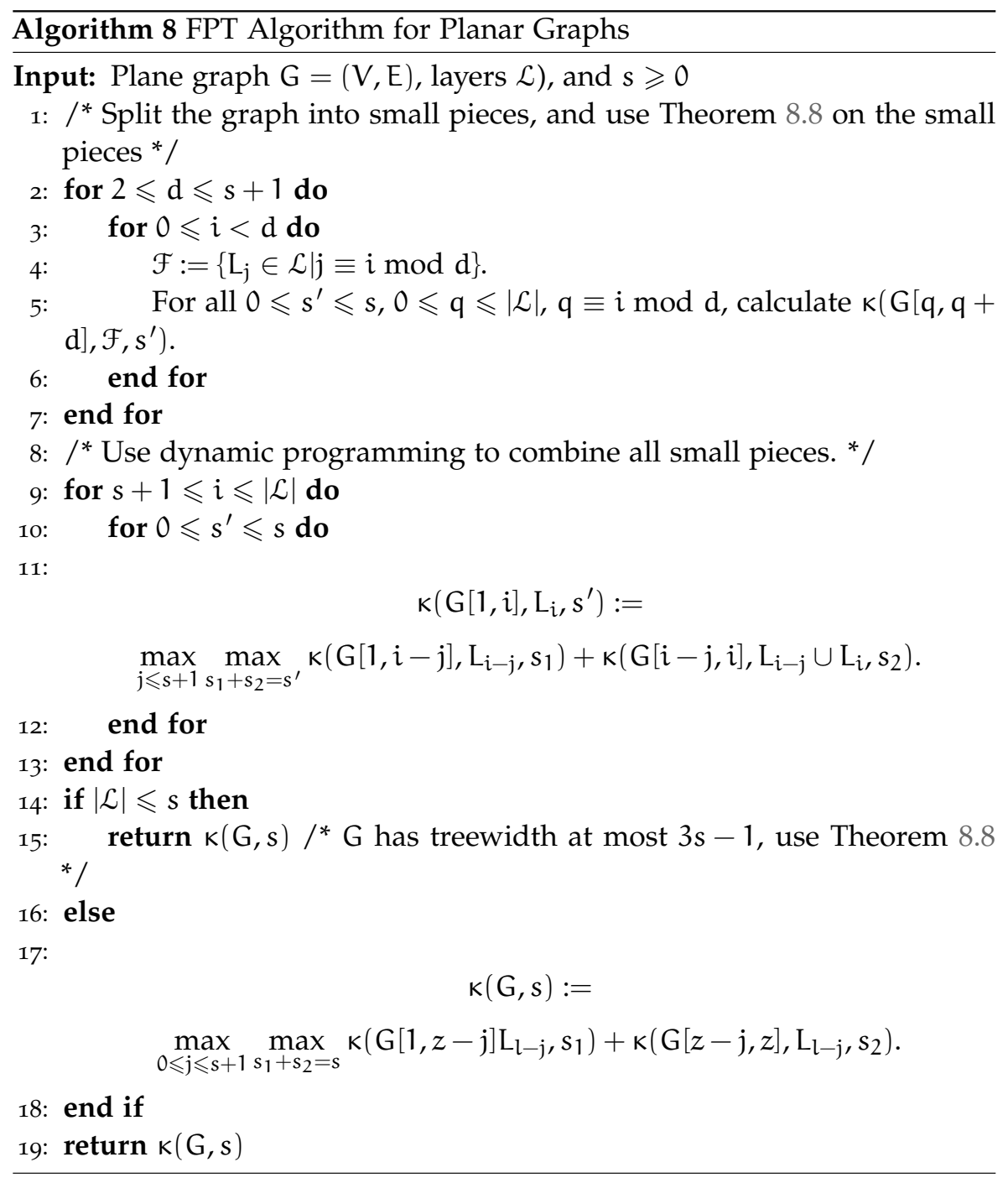

depending on $\varepsilon$. For a graph $\mathrm{G}$ and a cut-set $S$, the degree of a component $\mathrm{C} \in \mathrm{C}(\mathrm{G} \backslash \mathrm{S})$ is $|\mathrm{N}(\mathrm{C}) \cap \mathrm{S}|$.

Our approach is as follows. Using a threshold parameter $d$, we distinguish between components of low and high degree. Then, we analyze the high and low degree components separately. To approximate the maximum number of low degree components we make use of the decompo- 
sition Lemma 8.10. In combination with the Baker's type layer-shifting method [7], we arrive to an EPTAS for the number of low degree components. Secondly, in Lemma 8.14 we prove that in planar graphs there are not many components of high degree. Thus, by choosing the proper threshold parameter $d$ we ensure that the number of high degree components is at most $\varepsilon \cdot k(G, k)$. Combining both elements, we obtain an EPTAS for k-Way Vertex Cut.

In order to proceed further, we need several definitions. For a given cut-set $S$ and an integer $d>0$, we say a component from $\mathcal{C}(G \backslash S)$ is of low degree if it has at most $d$ neighbors in $S$. Let $c_{\leqslant d}(G, S)$ be the number of low degree components. Similarly, a component is of high degree if it has more than $d$ neighbors in $S$. We let $c_{>d}(G, S)$ be the number of high degree components. By definition, $c(G, S):=c_{\leqslant d}(G, S)+c_{>d}(G, S)$. We define $k_{\leqslant d}(G, k)$ to be the maximum of $c_{\leqslant d}(S)$ over all $S \subset V$ of cardinality at most $k$. Likewise, we define $\kappa_{>d}$. Clearly,

$$
\kappa(G, k) \leqslant \kappa \leqslant d(G, k)+\kappa_{>d}(G, k) .
$$

First, we approximate the number of low degree components $k_{\leqslant d}(G, k)$. Following the logic of the layer-shifting method of Baker, for some appropriate number $w$, we forbid every $w$-th layer to be in the cut-set $S$. Then, using Lemma 8.10 we can combine partial solutions obtained on the subgraphs between the forbidden layers. Choosing an appropriate shift of the forbidden layers, we guarantee that the combined solution contains at least $(1-\varepsilon) \kappa_{\leqslant d}(G, k)$ components.

To simplify the algorithm we use the following lemma. For a graph $G$ and forbidden layers $\mathcal{F}$ let $G_{\mathcal{F}}$ denote the graph $G$ after contracting every edge induced by $\mathcal{F}$ and let $F$ denote the set of vertices in $\mathrm{G}_{\mathcal{F}}$ that was involved in the contraction.

Lemma 8.12. Let $\mathrm{G}=(\mathrm{V}, \mathrm{E})$ be a plane graph, let $\mathrm{s} \geqslant 1$ be an integer and let $\mathcal{F}:=\left\{\mathrm{L}_{\mathrm{i}_{1}}, \ldots, \mathrm{L}_{\mathrm{i}_{\mathrm{r}}}\right\}$ be a set of forbidden layers as denoted above. Then,

1. $\kappa(G, \mathcal{F}, s)=\kappa\left(G_{\mathcal{F}}, F, s\right)$,

2. the treewidth of $\mathrm{G}_{\mathcal{F}}$ is at most $3\left(\max _{j=1}^{r-1}\left\{i_{j+1}-i_{j}\right\}+i_{1}+\left(z-i_{r}\right)\right)-1$.

Proof. For the first property, note that the solution does not change when edges betwen vertices not in the cut-set are contracted, therefore $k(G, \mathcal{F}, k)=$ $k\left(G_{\mathcal{F}}, F, k\right)$. The second property can be seen as follows: 
For convenience let $d:=\max _{j=1}^{r-1}\left\{i_{j+1}-i_{j}\right\}+i_{1}+\left(z-i_{r}\right)$. The graph $G_{\mathcal{F}}$ was obtained from $G$ by contracting every edge induced by $\mathcal{F}$, therefore every forbidden layer $L_{p}$ in $G$ corresponds to a vertex in $G_{\mathcal{F}}$. Decompose $G_{\mathcal{F}}$ into two-connected subgraphs. Every two-connected subgraph has at most $\mathrm{d}$ layers, and therefore has treewidth at most $3 \mathrm{~d}-1$ by Lemma 8.9. Moreover, for any two subgraphs $H$ and $H^{\prime}$ from this collection, $V(H) \cap V\left(H^{\prime}\right)$ is either an cut vertex or empty. Given a decomposition of $\mathrm{G}_{\mathcal{F}}$ into subgraphs, we find a tree decomposition for each subgraph separately. Obtained subgraph decompositions we straightforward amalgamate: consider two subgraph $H$ and $H^{\prime}$ sharing a single vertex $f \in F$ and let the corresponding tree decompositions be $(T, S)$ and $\left(T^{\prime}, S\right)$; find two nodes $t \in V(T)$ and $\mathrm{t}^{\prime} \in \mathrm{V}\left(\mathrm{T}^{\prime}\right)$ such that $v \in \mathrm{S}_{\mathrm{t}}$ and $v \in \mathrm{S}_{\mathrm{t}^{\prime}}$; connect $\mathrm{T}$ and $\mathrm{T}^{\prime}$ introducing an edge $e=\left(t, t^{\prime}\right)$; redefine $H:=\left(V(H) \cup V\left(H^{\prime}\right), E(H) \cup E\left(H^{\prime}\right) \cup\{e\}\right)$ and recurse on the remaining subgraphs disregarding $\mathrm{H}^{\prime}$. Finding the collection of subgraphs and amalgamation can be done in linear time.

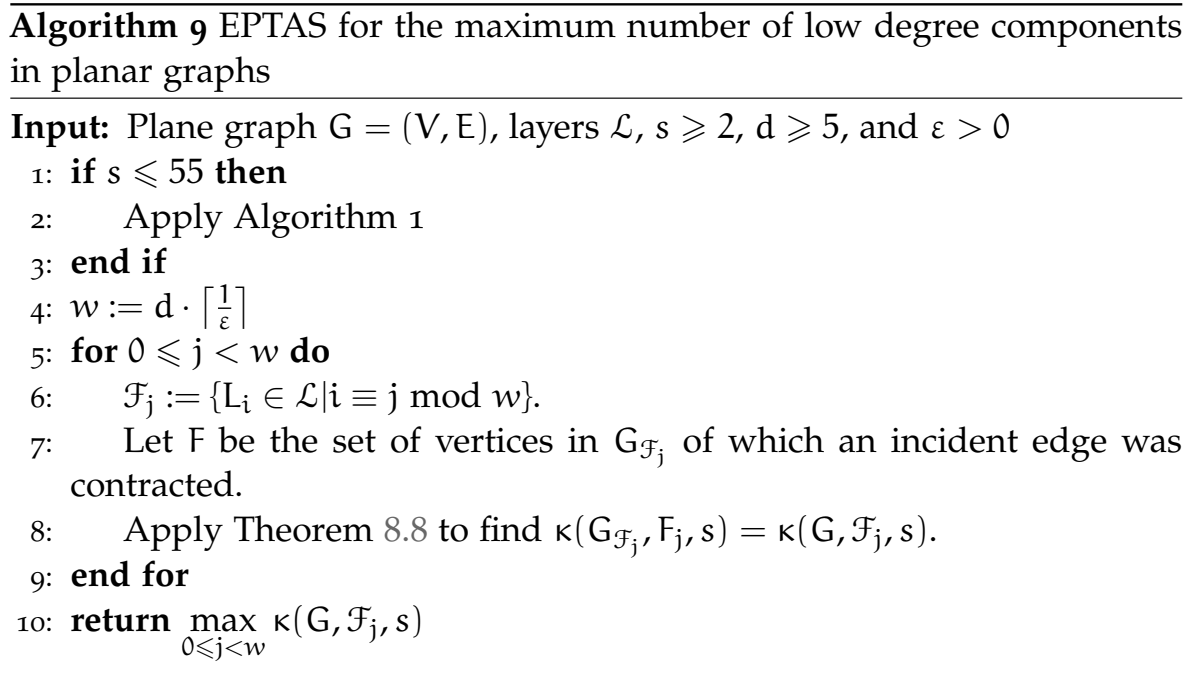

Lemma 8.13. Let $\mathrm{G}=(\mathrm{V}, \mathrm{E})$ be a planar graph, let $\mathrm{s} \geqslant 2$ and $\mathrm{d} \geqslant 5$ be integers and let $0<\varepsilon<1$ be a rational number. Then, Algorithm 9 is an $\mathrm{O}\left(\mathrm{ns}^{2}\left(\frac{\mathrm{d}}{\varepsilon}\right) \mathrm{O}\left(\frac{\mathrm{d}}{\varepsilon}\right)\right)$-time algorithm that approximates $\mathrm{K} \leqslant \mathrm{d}(\mathrm{G}, \mathrm{s})$ within a factor of $(1-\varepsilon)$. 
Proof. First, the fact that we can apply Theorem 8.8 in line 8 follows from Lemma 8.12. Let the distance between two consecutive forbidden layers be $w:=\mathrm{d}\left\lceil\frac{1}{\varepsilon}\right\rceil$. For any (integer) shift $j, 0 \leqslant j<w$, let $V_{j}:=\bigcup\left\{L_{i} \in\right.$ $\mathcal{L}(\mathrm{G}) \mid i \equiv j \bmod w\}$. Let $S^{\mathrm{OPT}} \subset \mathrm{V}$ be the optimal cut-set. We say that two distinct components in $\mathrm{G}\left[\mathrm{V} \backslash \mathrm{S}^{\mathrm{OPT}}\right]$ break by $\mathrm{V}_{j}$ if they become connected in $\mathrm{G}\left[\mathrm{V} \backslash\left(S^{\mathrm{OPT}} \backslash V_{j}\right)\right]$. The solution that our algorithm returns is therefore at least $c_{\leqslant d}\left(G, S^{O P T} \backslash V_{j}\right)$.

Let $b_{j}$ be the number of components in $G\left[V \backslash S^{O P T}\right]$ that break by $V_{j}$. Then, by definition of $b_{j}$

$$
c_{\leqslant d}\left(G, S^{O P T} \backslash V_{j}\right) \geqslant c_{\leqslant d}\left(G, S^{O P T}\right)-b_{j} .
$$

Observe that a component can only break by $V_{j}$ if it has a neighboring vertex in $S^{\mathrm{OPT}} \cap V_{j}$. Since we consider only components of degree at most $d$, there are at most $d$ values $i_{1}, \ldots, i_{d}$ such that a component is broken by $v_{i_{1}}, \ldots, V_{i_{d}}$. Therefore,

$$
\sum_{j=0}^{w-1} b_{j} \leqslant d \cdot k_{\leqslant d}(G, s) .
$$

Let $b_{\min }:=\min _{s=0, \ldots, w-1} b_{j}$. From Equation 53 and standard averaging argument

$$
b_{\min } \leqslant \frac{d \cdot k_{k d}(G, s)}{w}=\frac{d \cdot k_{k d}(G, s)}{d \cdot\left\lceil\frac{1}{\varepsilon}\right\rceil} \leqslant \varepsilon \cdot \kappa_{\leqslant d}(G, s) .
$$

Combining Equations 52 and 54 we derive that Algorithm 9 returns a cutset splitting the graph in at least $(1-\varepsilon) \kappa_{\leqslant d}(G, s)$ components.

It remains to analyze the running time of Algorithm 9. In line 2 we invoke Algorithm 1, which takes $\mathrm{O}(\mathrm{n})$ time. In lines 5-8 we invoke Theorem $8.8 w$ times, which takes in total $\mathrm{O}\left(w \cdot n s^{2}\left(\frac{\mathrm{d}}{\varepsilon}\right)^{\mathrm{O}\left(\frac{\mathrm{d}}{\varepsilon}\right)}\right)$ time.

Now, we estimate the number of the high degree components $K_{>d}(G, s)$.

Lemma 8.14. Let $\mathrm{G}=(\mathrm{V}, \mathrm{E})$ be a planar graph, and let $\mathrm{k} \geqslant 2$ and $\mathrm{d} \geqslant 3$ be integers. Then, $\mathrm{K}_{>\mathrm{d}}(\mathrm{G}, \mathrm{s}) \leqslant \frac{\mathrm{s}-4}{\mathrm{~d}-2}$.

Proof. Let $m$ be the number of edges in $G$, and let $f$ be the number of faces in an embedding of $\mathrm{G}$. Let $\mathrm{S} \subset \mathrm{V}$ be an arbitrary cut-set. Consider a minor $H=\left(V^{\prime}, E^{\prime}\right)$ obtained from $G$ by: 
1. Contracting all pairs of adjacent vertices that are not in S;

2. Removing all vertices of degree less than $d$ that are not in the cut-set;

3. Removing all edges between cut-set vertices;

4. For each component $T$ of degree more than $d$, remove arbitrary edges between $T$ and $S$ such that the degree of $T$ becomes $d$.

In the obtained minor $\mathrm{H}$, each vertex not in $\mathrm{S}$ is called a component vertex, and each component in $\mathrm{G}[\mathrm{V} \backslash \mathrm{S}$ ] of degree at least $\mathrm{d}$ has a corresponding component vertex in $\mathrm{H}$. By construction, $\mathrm{H}$ is bipartite, all component vertices have degree $d$. Therefore, $n^{\prime}=\left|V^{\prime}\right|=|S|+c_{>d}(G, S)$ and $m^{\prime}=$ $\left|E^{\prime}\right|=d \cdot c_{>d}(G, S)$. Denote by $f^{\prime}$ the number of faces in a planar embedding of $\mathrm{H}$. As $\mathrm{H}$ is bipartite every face is incident to at least four edges, and we can conclude that $f^{\prime} \leqslant m^{\prime} / 2$. By Euler's formula,

$$
2=n^{\prime}-m^{\prime}+f^{\prime} \leqslant s+c_{>d}(G, S)-d \cdot c_{>d}(G, S)+d \cdot c_{>d}(G, S) / 2 .
$$

Thus, $k_{>d}(G, s) \leqslant \frac{2 s-4}{d-2}$.

The final ingredient needed for our main result is the following lemma. This lemma provides a simple lower bound on the total number of components $k(G, s)$.

Lemma 8.15. Let $\mathrm{G}=(\mathrm{V}, \mathrm{E})$ be a planar graph and let $\mathrm{s} \leqslant \frac{5}{6} \mathrm{n}$. Then, $\left\lfloor\frac{\mathrm{s}}{5}\right\rfloor \leqslant$ $K(G, s)$.

Proof. It is well known that any simple planar graph has a vertex $v \in \mathrm{V}$ of degree at most 5 . Set an initial cut-set $S=\emptyset$. Find a non-isolated vertex $v$ of the lowest degree in $\mathrm{G}[\mathrm{V} \backslash \mathrm{S}]$. Consider the neighborhood $\mathrm{N}(v)$ of $v$ in this graph. If $|S \cup N(v)| \leqslant s$, then define $S:=S \cup N(v)$. Since $s \leqslant \frac{5}{6} n$, we can make $\left\lfloor\frac{s}{5}\right\rfloor$ steps of this procedure, obtaining a cut-set $S$ of at most $s$ vertices while the graph $\mathrm{G}[\mathrm{V} \backslash \mathrm{S}]$ has at least $\left\lfloor\frac{\mathrm{s}}{5}\right\rfloor$ components.

Now we are ready to present our main theorem, an efficient polynomialtime approximation scheme for k-WAy Vertex Cut.

Theorem 8.16. Let $\mathrm{G}=(\mathrm{V}, \mathrm{E})$ be a planar graph, let $\mathrm{s} \geqslant 2$ be an integer and let $0<\varepsilon<1$ be a rational number. Then, there is a $\mathrm{O}\left(\mathrm{ns}^{2}\left(\frac{1}{\varepsilon^{2}}\right)^{\mathrm{O}\left(\frac{1}{\varepsilon^{2}}\right)}\right)$ time algorithm that approximates $\mathrm{K}(\mathrm{G}, \mathrm{s})$ within a factor of $(1-\varepsilon)$. 
Proof. Define $\delta:=\frac{\varepsilon}{2-\varepsilon}$. If $s<55$ we apply the FPT algorithm in Theorem 8.11 and we find the optimal solution in linear time.

For $55 \leqslant s$, we observe the following. If $\mathrm{S} \subset \mathrm{V}$ is a cut-set of cardinality $\left\lfloor\frac{5}{6} n\right\rfloor+j \leqslant s$ where $j>0$, then $c(G, S) \leqslant n-\left\lfloor\frac{5}{6} n\right\rfloor-j=\left\lceil\frac{1}{6} n\right\rceil-j$. By Lemma 8.15, $\left\lfloor\frac{\left\lfloor\frac{5}{6} n\right\rfloor}{5}\right\rfloor=\left\lfloor\frac{1}{6} n\right\rfloor \leqslant \kappa\left(G, \frac{5}{6} n\right)$. Hence, we can assume $55 \leqslant s \leqslant$ $\frac{5}{6} n$.

Define $d:=\left\lceil\frac{24}{\delta}\right\rceil$. Consider two solutions to k-Way Vertex Cut. The first cut-set is generated by the algorithm in Lemma 8.15, and the second cut-set is returned by Algorithm 9 with parameters $d$ and $\delta$. From these two cut-sets, we choose the cut-set that splits the graph into the most components. Let the resulting number of components be denoted by ALG. By Lemmas 8.13 and 8.15 , and by definition of ALG,

$$
A L G \geqslant \max \left\{(1-\delta) \kappa_{\leqslant d}(G, s),\left\lfloor\frac{s}{5}\right\rfloor\right\} .
$$

Thus, from Lemma 8.14 and Equation 55 we obtain

$$
\kappa_{>d}(G, s) \leqslant \frac{2 s-4}{d-2} \leqslant \frac{2 s}{22} \delta \leqslant\left\lfloor\frac{s}{5}\right\rfloor \delta \leqslant \delta A L G .
$$

Combining Equations 51, 55 and 56, we derive

$$
\begin{aligned}
(1-\varepsilon) \kappa(G, s) & \leqslant(1-\varepsilon) \kappa_{\leqslant d}(G, s)+(1-\varepsilon) \kappa_{>d}(G, s) \\
& \leqslant A L G .
\end{aligned}
$$

The running time for obtaining the solution in Lemma 8.13 clearly dominates the running time for obtaining the solution in Lemma 8.15. Therefore, the time requirement in the theorem will be the same as in Lemma 8.13, making the total running time

$$
n s^{2}\left(\frac{\mathrm{d}}{\delta}\right)^{\mathrm{O}\left(\frac{\mathrm{d}}{\delta}\right)}=n s^{2}\left(\frac{\left\lceil\frac{24}{\delta}\right\rceil}{\delta}\right)^{\mathrm{O}\left(\frac{\left\lceil\frac{24}{\delta}\right\rceil}{\delta}\right)}=n s^{2}\left(\frac{1}{\varepsilon^{2}}\right)^{\mathrm{O}\left(\frac{1}{\varepsilon^{2}}\right)} .
$$

\subsection{CONCLUSIONS}

The main result of this chapter was an EPTAS for k-WAy VerTex Cut, a natural graph separation problem which surprisingly did not receive much 
attention in the literature. Further, we identified two graph classes for which k-Way VerTex Cut is polynomially time solvable, namely interval graphs and graphs of bounded treewidth. However, k-WAy Vertex Cut is W[1]-hard on split graphs and does not allow a PTAS under a reasonable complexity assumption.

There are several questions remaining. Are there PTASs for graphs of bounded genus and graphs with an excluded minor? 


\section{BIBLIOGRAPHY}

[1] A. Akella, B. Maggs, S. Seshan, A. Shaikh, and R. Sitaraman. "A measurement-based analysis of multihoming." In: ACM SIGCOMM 2003 Conference on Applications, Technologies, Architectures, and Protocols for Computer Communication (SIGCOMM). 2003, pages 353-364 (cit. on p. 109).

[2] N. Alon, D. Moshkovitz, and S. Safra. "Algorithmic construction of sets for k-restrictions". In: ACM Transactions on Algorithms 2.2 (2006), pp. 153-177 (cit. on p. 22).

[3] S. Arora, B. Barak, and D. Steurer. "Subexponential Algorithms for Unique Games and Related Problems". In: Proceedings of the 47th IEEE Symposium on Foundations of Computer Science (FOCS) (2010), pp. 563-572 (cit. on p. 14).

[4] S. Arora, D. Karger, and M. Karpinski. "Polynomial time approximation schemes for dense instances of np-hard problems". In: Journal of Computer and System Sciences 58.1 (1999), pp. 193-210 (cit. on p. 128).

[5] Y. Azar and I. Gamzu. "Ranking with Submodular Valuations". In: 22nd Annual ACM-SIAM Symposium on Discrete Algorithms (SODA). 2011, pp. 1070-1079 (cit. on pp. 24, 26, 66-70, 72, 75, 80, 81, 86).

[6] Y. Azar, I. Gamzu, and X. Yin. "Multiple intents re-ranking". In: 41st Annual ACM Symposium on Theory of Computin (STOC). 2009, pp. $669-678$ (cit. on pp. $23,26,66,68,88$ ).

[7] B.S. Baker. "Approximation algorithms for NP-complete problems on planar graphs". In: Journal of the ACM 41.1 (1994), pp. 153-180 (cit. on pp. 139, 141).

[8] N. Bansal, A. Gupta, and R. Krishnaswamy. "A Constant Factor Approximation Algorithm for Generalized Min-Sum Set Cover". In: 21st Annual ACM-SIAM Symposium on Discrete Algorithms (SODA). 2010, pp. 1539-1545 (cit. on pp. 23, 26, 30, 31, 59, 66, 68, 80, 88, 89).

[9] N. Bansal and S. Khot. "Optimal Long Code Test with One Free Bit". In: 5oth Annual IEEE Symposium on Foundations of Computer Science (FOCS). 2009, pp. 453-462 (cit. on pp. 14, 24, 30, 49). 
[10] A. Bar-Noy, M. Bellare, M.M. Halldórsson, H. Shachnai, and T. Tamir. "On Chromatic Sums and Distributed Resource Allocation". In: Information and Computation 140.2 (1998), pp. 183-202 (cit. on p. 23).

[11] A. Bar-Noy, M. Bellare, M.M. Halldórsson, H. Shachnai, and T. Tamir. “On Chromatic Sums and Distributed Resource Allocation". In: Information and Computation 140.2 (1998), pp. 183-202 (cit. on p. 68).

[12] A. Bar-Noy, M.M. Halldórsson, and G. Kortsarz. "A Matched Approximation Bound for the Sum of a Greedy Coloring". In: Information Processing Letters 71.3 (1999), pp. 135-140 (cit. on p. 23).

[13] R. Beier, H. Röglin, and B. Vöcking. "The smoothed number of Pareto optimal solutions in bicriteria integer optimization". In: Integer Programming and Combinatorial Optimization, 12th International Conference (IPCO). 2007, pp. 53-67 (cit. on pp. 116, 124).

[14] A. Berger, A. Grigoriev, and R. van der Zwaan. "How to Cut a Graph into Many Pieces". In: Theory and Applications of Models of Computation - 8th Annual Conference (TAMC). 2011, pp. 184-194 (cit. on p. 127).

[15] A. Berger, H. Röglin, and R. van der Zwaan. "Path Trading: Fast Algorithms, Smoothed Analysis, and Hardness Results". In: Experimental Algorithms - 1oth International Symposium (SEA). 2011, pp. 4353 (cit. on p. 107).

[16] A. Bhaskara, M. Charikar, E. Chlamtac, U. Feige, and A. Vijayaraghavan. "Detecting high log-densities: an $\mathrm{n}^{1 / 4}$ approximation for densest k-subgraph". In: 42nd ACM Symposium on Theory of Computing (STOC). 2010, pp. 201-210 (cit. on p. 75).

[17] H.L. Bodlaender. "A linear-time algorithm for finding tree-decompositions of small treewidth". In: SIAM Journal on Computing 25.6 (1996), pp. 13051317 (cit. on p. 133).

[18] H.L. Bodlaender. "A partial k-arboretum of graphs with bounded treewidth". In: Theoretical Computer Science 209.1-2 (1998), pp. 1-45 (cit. on p. 136).

[19] K. Booth and G. Lueker. "Testing for the consecutive ones property, interval graphs, and graph planarity using pq-tree algorithms". In: Journal of Computer and System Sciences 13.3 (1976), pp. 335-379 (cit. on p. 132). 
[20] S. Burer and R. Monteiro. "A projected gradient algorithm for solving the Maxcut SDP relaxation". In: Optimization Methods and Software 15 (2001), pp. 175-200 (cit. on p. 23).

[21] G. Calinescu and A. Zelikovsky. "The Polymatroid Steiner Problems". In: Journal of Combinatorial Optimization 9.3 (2005), pp. 281294 (cit. on pp. 27, 67, 75).

[22] R.D. Carr, L. Fleischer, V.J. Leung, and C.A. Phillips. "Strengthening integrality gaps for capacitated network design and covering problems". In: 11th Annual ACM-SIAM Symposium on Discrete Algorithms (SODA). 2000, pp. 106-115 (cit. on p. 89).

[23] D. Chakrabarty and C. Swamy. "Facility Location with Client Latencies: Linear Programming Based Techniques for Minimum Latency Problems". In: Integer Programming and Combinatoral Optimization 15th International Conference (IPCO). 2011, pp. 92-103 (cit. on pp. 28, 88).

[24] M. Charikar, C. Chekuri, and M. Pál. "Sampling Bounds for Stochastic Optimization". In: Approximation, Randomization and Combinatorial Optimization, Algorithms and Techniques, 8th International Workshop on Approximation Algorithms for Combinatorial Optimization Problems and 9th International Workshop on Randomization and Computation (APPROX/RANDOM). 2005, pp. 257-269 (cit. on p. 80).

[25] K. Chaudhuri, B. Godfrey, S. Rao, and K. Talwar. "Paths, Trees, and Minimum Latency Tours". In: 44th Symposium on Foundations of Computer Science (FOCS). 2003, pp. 36-45 (cit. on pp. 68, 75).

[26] C. Chekuri, G. Even, and G. Kortsarz. "A greedy approximation algorithm for the group Steiner problem". In: Discrete Applied Mathematics 154.1 (2006), pp. 15-34 (cit. on pp. 27, 67).

[27] C. Chekuri and R. Motwani. "Precedence Constrained Scheduling to Minimize Sum of Weighted Completion Times on a Single Machine". In: Discrete Applied Mathematics 98.1-2 (1999), pp. 29-38 (cit. on p. 23).

[28] C. Chekuri and M. Pál. "A Recursive Greedy Algorithm for Walks in Directed Graphs". In: 46th Annual IEEE Symposium on Foundations of Computer Science (FOCS). 2005, pp. 245-253 (cit. on pp. 27, 67, 75).

[29] J. Chen, Y. Liu, and S. Lu. "An Improved Parameterized Algorithm for the Minimum Node Multiway Cut Problem". In: Algorithmica 55.1 (2009), pp. 1-13 (cit. on p. 128). 
[30] M. Costa, L. Létocart, and F. Roupin. "Minimal multicut and maximal integer multiflow: A survey". In: European Journal of Operational Research 162 (2005), pp. 55-69 (cit. on p. 128).

[31] G. Călinescu, C. Fernandes, and B. Reed. "Multicuts in unweighted graphs and digraphs with bounded degree and bounded tree-width". In: Journal of Algorithms 48.4 (2003), pp. 333-359 (cit. on p. 128).

[32] G. Călinescu, H. Karloff, and Y. Rabani. "An improved approximation algorithm for multiway cut". In: Journal of Computer and System Sciences 60.3 (2000), pp. 564-574 (cit. on p. 128).

[33] E. Dahlhaus, D. S. Johnson, C. H. Papadimitriou, P. D. Seymour, and M. Yannakakis. "The complexity of multiterminal cuts". In: SIAM Journal on Computing 23 (1994), pp. 864-894 (cit. on p. 128).

[34] R. Dai, D. O. Stahl, and A. B. Whinston. "The economics of smart routing and QoS." In: Group Communications and Charges; Technology and Business Models, 5th COST264 International Workshop on Networked Group Communications (NGC). 2003, pp. 318-331 (cit. on p. 109).

[35] R. Downey, V. Estivill-Castro, M. Fellows, E. Prieto, and F. Rosamond. "Cutting up is hard to do: the parameterized complexity of k-cut and related problems". In: Electronic Notes in Theoretical Computer Science 78 (2003) (cit. on p. 128).

[36] R.G. Downey and M.R. Fellows. Parameterized Complexity. SpringerVerlag, Berlin, 1999 (cit. on p. 12).

[37] I. Dunur and S. Safra. "On the hardness of approximating minimum vertex cover". In: Annals of Mathematics 162.1 (2005), pp. 439-485 (cit. on p. 13).

[38] J. Fakcharoenphol, C. Harrelson, and S. Rao. “The k-traveling repairmen problem". In: ACM Transactions on Algorithms 3.4 (2007) (cit. on pp. 68, 75).

[39] J. Fakcharoenphol, S. Rao, and K. Talwar. "A tight bound on approximating arbitrary metrics by tree metrics". In: Journal of Computer and System Sciences 69.3 (2004), pp. 485-497 (cit. on pp. 90, 103).

[40] U. Feige. "A Threshold of $\ln n$ for Approximating Set Cover". In: Journal of the ACM 45.4 (1998), pp. 634-652 (cit. on pp. 11, 22).

[41] U. Feige, L. Lovász, and P. Tetali. "Approximating Min Sum Set Cover". In: Algorithmica 40.4 (2004), pp. 219-234 (cit. on pp. 23, 51, 68). 
[42] M. Garey and D. Johnson. Computers and Intractability: A Guide to the Theory of NP-Completeness. W. H. Freeman, 1979 (cit. on p. 130).

[43] N. Garg, G. Konjevod, and R. Ravi. "A Polylogarithmic Approximation Algorithm for the Group Steiner Tree Problem". In: Journal of Algorithms 37.1 (2000), pp. 66-84 (cit. on pp. 26, 27, 66, 67).

[44] N. Garg, V. Vazirani, and M. Yannakakis. "Multiway cuts in node weighted graphs". In: Journal of Algorithms 50.1 (2004), pp. 49-61 (cit. on pp. 127, 128).

[45] N. Garg, V. Vazirani, and M. Yannakakis. "Primal-dual approximation algorithms for integral flow and multicut in trees". In: Algorithmica 18 (1997), pp. 3-20 (cit. on p. 128).

[46] M.X. Goemans and J. Vondrák. "Stochastic Covering and Adaptivity". In: Theoretical Informatics, 7 th Latin American Symposium (LATIN). 2006, pp. 532-543 (cit. on pp. 25, 68, 69, 79).

[47] M.X. Goemans and D.P. Williamson. "Improved approximation algorithms for maximum cut and satisfiability problems using semidefinite programming". In: Journal of the ACM 42.6 (1995), 1115Ü-1145 (cit. on p. 13).

[48] D.K. Goldenberg, L. Qiu, H. Xie, Y. R. Yang, and Y. Zhang. “Optimizing cost and performance for multihoming". In: ACM SIGCOMM 2004 Conference on Applications, Technologies, Architectures, and Protocols for Computer Communication (SIGCOMM). 2004, pp. 79-82 (cit. on p. 109).

[49] O. Goldschmidt and D. Hochbaum. "Polynomial algorithm for the k-cut problem for fixed k". In: Mathematics of Operations Research 19.1 (1994), pp. 24-37 (cit. on p. 128).

[50] D. Golovin and A. Krause. "Adaptive Submodularity: A New Approach to Active Learning and Stochastic Optimization". In: 23rd Conference on Learning Theory (COLT). 2010, pp. 333-345 (cit. on pp. 25, 68).

[51] M. Grötschel, L. Lovász, and A. Schrijver. Geometric algorithms and combinatorial optimization. Second edition. Algorithms and Combinatorics, 2. Springer-Verlag, Berlin, 1993 (cit. on pp. 41, 43). 
[52] J. Guo, F. Hüffner, E. Kenar, R. Niedermeier, and J. Uhlmann. “Complexity and exact algorithms for vertex multicut in interval and bounded treewidth graphs". In: European Journal of Operational Research 186.2 (2008), pp. 542-553 (cit. on p. 128).

[53] A. Gupta, V. Nagarajan, and R. Ravi. "Approximation Algorithms for Optimal Decision Trees and Adaptive TSP Problems". In: $A u-$ tomata, Languages and Programming, 37th International Colloquium (ICALP). 2010, pp. 690-701 (cit. on pp. 28, 88).

[54] A. Gupta and A. Srinivasan. "An Improved Approximation Ratio for the Covering Steiner Problem". In: Theory of Computing 2.1 (2006), pp. 53-64 (cit. on pp. 27, 88-90, 94, 95, 98).

[55] N. Guttmann-Beck and R. Hassin. "Approximation algorithms for minimum k-cut". In: Algorithmica 27.2 (2000), pp. 198-207 (cit. on p. 128).

[56] M. Hajiaghayi and M. Hajiaghayi. "A note on the bounded fragmentation property and its applications in network reliability". In: European Journal of Combinatorics 24.7 (2003), pp. 891-896 (cit. on p. 128).

[57] L.A. Hall, A.S. Schulz, D.B. Shmoys, and J. Wein. "Scheduling to Minimize Average Completion Time: Off-Line and On-Line Approximation Algorithms". In: Mathematics of Operations Research 22.3 (1997), pp. 513-544 (cit. on p. 23).

[58] E. Halperin and R. Krauthgamer. "Polylogarithmic inapproximability". In: 35th Annual ACM Symposium on Theory of Computing (STOC). 2003, pp. 585-594 (cit. on p. 27).

[59] X. He. "An improved algorithm for the planar 3-cut problem". In: Journal of Algorithms 12.1 (1991), pp. 23-37 (cit. on p. 128).

[6o] D.S. Hochbaum and D. Shmoys. "An $\mathrm{O}\left(|\mathrm{V}|^{2}\right)$ algorithm for the planar 3-cut problem". In: SIAM Journal on Algebraic and Discrete Methods 6 (1985), pp. 707-712 (cit. on p. 128).

[61] J. Hopcroft and R. Tarjan. "Efficient planarity testing". In: Journal of the ACM 21.4 (1974), pp. 549-568 (cit. on p. 136).

[62] J. Håstad. "Some optimal inapproximability results". In: Journal of the $A C M 48.4$ (2001), 798 Ú-859 (cit. on p. 13). 
[63] S. Im, V. Nagarajan, and R. van der Zwaan. "Minimum Latency Submodular Cover". In: Automata, Languages, and Programming - 39th International Colloquium (ICALP) (2012), pp. 485-497 (cit. on pp. 65, 87).

[64] S. Im, M. Sviridenko, and R. van der Zwaan. "Preemptive and nonpreemptive generalized min sum set cover". In: 29th International Symposium on Theoretical Aspects of Computer Science (STACS). 2012, pp. $465-476$ (cit. on p. 29).

[65] R. Impagliazzo and R. Paturi. "On the Complexity of k-SAT". In: Journal of Computer and System Sciences 62.2 (2001), pp. 367-375 (cit. on p. II).

[66] Y. Kamidoi, N. Yoshida, and H. Nagamochi. "A deterministic algorithm for finding all minimum k-way cuts". In: SIAM Journal on Computing 36.5 (2006), pp. 1329-1341 (cit. on p. 128).

[67] D.R. Karger and C. Stein. "A new approach to the minimum cut problem". In: Journal of the ACM 43.4 (1996), pp. 601-640 (cit. on p. 128).

[68] K. Kawarabayashi and M. Thorup. "Minimum k-way cut of bounded size is fixed-parameter tractable". In: 52nd Annual Symposium on Foundations of Computer Science (FOCS). 2011 (cit. on p. 128).

[69] S. Khot. "Inapproximability of NP-complete Problems, Discrete Fourier Analysis, and Geometry". In: Proceedings of the International Congress of Mathematicians (2010) (cit. on p. 14).

[70] S. Khot. "Ruling out PTAS for graph min-bisection, dense k-subgraph, and bipartite clique". In: SIAM Journal on Computing 36.4 (2006), pp. 1025-1071 (cit. on pp. 10, 131).

[71] S. Khot, G. Kindler, E. Mossel, and R. O'Donell. "Optimal inapproximability results for MAX-CUT and other 2-variable CSPs". In: SIAM Journal on Computing 37.1 (2007), 319Ü-357 (cit. on p. 13).

[72] S. Khot and O. Regev. "Vertex cover might be hard to approximate to within $2-\varepsilon$ ". In: Journal of Computer and System Sciences 74.3 (2008), 335Ü-349 (cit. on p. 13).

[73] A.J. Kleywegt, A. Shapiro, and T. Homem de Mello. "The Sample Average Approximation Method for Stochastic Discrete Optimization". In: SIAM Journal on Optimization 12.2 (2002), pp. 479-502 (cit. on p. 80). 
[74] T. Kloks. Treewidth, Computations and Approximations. Vol. 842. Lecture Notes in Computer Science, volume 842, 1994 (cit. on p. 133).

[75] D. Knuth. The Art of Computer Programming, Volume 3: Sorting and Searching, Third Edition. Addison-Wesley, 1997 (cit. on p. 116).

[76] G. Konjevod, R. Ravi, and A. Srinivasan. "Approximation algorithms for the covering Steiner problem". In: Random Structures and Algorithms 20.3 (2002), pp. 465-482 (cit. on pp. 27, 88-90, 94, 95).

[77] Y. Liu and A.L.N. Reddy. "Multihoming route control among a group of multihomed stub networks." In: Computer Communications 30.17 (2007), pp. 3335-3345 (cit. on pp. 107, 109).

[78] Z. Liu, S. Parthasarathy, A. Ranganathan, and H. Yang. "Near-optimal algorithms for shared filter evaluation in data stream systems". In: ACM SIGMOD International Conference on Management of Data (SIGMOD). 2008, pp. 133-146 (cit. on pp. 25, 69, 79).

[79] R. Mahajan, D. Wetherall, and T. Anderson. "Negotiation-based routing between neighboring ISPs". In: 2nd Symposium on Networked Systems Design and Implementation (NSDI). 2005, pp. 29-42 (cit. on pp. 107, 109, 120, 124).

[8o] F. Margot, M. Queyranne, and Y. Wang. "Decompositions, Network Flows, and a Precedence Constrained Single-Machine Scheduling Problem". In: Operations Research 51.6 (2003), pp. 981-992 (cit. on p. 23).

[81] D. Marx. "Parameterized graph separation problems". In: Theoretical Computer Science 351 (2006), pp. 394-406 (cit. on pp. 127, 129, 131).

[82] R. Motwani and P. Raghavan. Randomized Algorithms. Cambridge University Press, 1995 (cit. on p. 55).

[83] K. Munagala, U. Srivastava, and J. Widom. "Optimization of continuous queries with shared expensive filters". In: 27th ACM SIGMODSIGACT-SIGART Symposium on Principles of Database Systems (PODS). 2007, pp. 215-224 (cit. on pp. 25, 69, 79).

[84] V. Nagarajan. "Approximation Algorithms for Sequencing Problems". $\mathrm{PhD}$ thesis. Tepper School of Business, Carnegie Mellon University, 2009 (cit. on pp. 28, 88).

[85] G.L. Nemhauser and Z. Ullmann. "Discrete dynamic programming and capital allocation". In: Management Science 15.9 (1969), pp. 494505 (cit. on p. 115). 
[86] M. Queyranne and M. Sviridenko. "A $(2+\varepsilon)$-approximation algorithm for the generalized preemptive open shop problem with minsum objective". In: J. Algorithms 45.2 (2002), pp. 202-212 (cit. on p. 45).

[87] B. Quoitin and O. Bonaventure. "A cooperative approach to interdomain traffic engineering". In: (2005) (cit. on p. 109).

[88] P. Raghavendra. "Optimal Algorithms and Inapproximability Results for Every CSP?" In: Proceedings of the 4oth Annual ACM Symposium on Theory of Computing (STOC) (2009), pp. 245-254 (cit. on p. 14).

[89] R. Raz and S. Safra. "A Sub-Constant Error-Probability Low-Degree Test, and a Sub-Constant Error-Probability PCP Characterization of NP". In: Proceedings of the Twenty-Ninth Annual ACM Symposium on the Theory of Computing (STOC) (1997), pp. 475-484 (cit. on p. 22).

[9o] H. Röglin and S.-H. Teng. "Smoothed Analysis of Multiobjective Optimization". In: 5oth Annual IEEE Symposium on Foundations of Computer Science (2009). 2009, pp. 681-690 (cit. on p. 124).

[91] H. Saran and V. Vazirani. "Finding k cuts within twice the optimal". In: SIAM Journal on Computing 24.1 (1995), pp. 101-108 (cit. on p. 128).

[92] A. Schrijver. Combinatorial optimization: polyhedra and efficiency. SpringerVerlag, Berlin, 2003 (cit. on pp. 17, 18, 36, 43).

[93] A. Schulz and M. Skutella. "Random-based scheduling: new approximations and LP lower bounds". In: Randomization and Approximation Techniques in Computer Science, International Worksho (RANDOM). 1997, pp. 119-133 (cit. on p. 45).

[94] P. Sevcik and J. Bartlett. "Improving user experience with route control". In: Technical Report NetForecast Report 5062, NetForecast, Inc. (2002) (cit. on p. 109).

[95] Y. Shavitt and E. Shir. "DIMES: let the Internet measure itself". In: ACM SIGCOMM Computer Communication Review 35.5 (2005), pp. 7174 (cit. on pp. 108, 120).

[96] Y. Shavitt and Y. Singer. "Limitations and Possibilities of Path Trading between Autonomous Systems". In: 29th IEEE International Conference on Computer Communications (INFOCOM). 2010 (cit. on pp. 107, 111, 120, 124). 
[97] G. Shrimali, A. Akella, and A. Mutapcic. "Cooperative interdomain traffic engineering using nash bargaining and decomposition". In: 26th IEEE International Conference on Computer Communications (INFOCOM). 2007, pp. 330-338 (cit. on pp. 109, 120).

[98] M. Skutella and D.P. Williamson. "A note on the generalized minsum set cover problem". In: Operations Research Letters 39.6 (2011), pp. 433-436 (cit. on pp. 23, 30, 31, 51, 53, 55, 59, 80).

[99] D. A. Spielman and S.-H. Teng. "Smoothed analysis: an attempt to explain the behavior of algorithms in practice". In: Communications of the ACM 52.10 (2009), pp. 76-84 (cit. on p. 108).

[10o] D.A. Spielman and S.-H. Teng. "Smoothed analysis of algorithms: why the simplex algorithm usually takes polynomial time". In: Journal of the ACM 51.3 (2004), pp. 385-463 (cit. on p. 108).

[101] R. Teixeira, A. Shaikh, T. Griffin, and J. Rexford. "Dynamics of hotpotato routing in IP networks". In: International Conference on Measurements and Modeling of Computer Systems (SIGMETRICS). 2004, pp. 307-319 (cit. on p. 107).

[102] M. Thorup. "Minimum k-way cuts via deterministic greedy tree packing". In: 4oth Annual ACM Symposium on Theory of Computing (STOC). 2008 (cit. on p. 128).

[103] L. Trevisan. “On Khot's Unique Games Conjecture". In: Bulletin (New Series) of the American Mathematical Society 49.1 (2012), 91Ü-111 (cit. on p. 14).

[104] L. Trevisan, G. Sorkin, M. Sudan, and D. Williamson. "Gadgets, Approximation, and Linear Programming". In: Proceedings of the 37th IEEE Symposium on Foundations of Computer Science (FOCS) (2000), 617Ü-626 (cit. on p. 13).

[105] J. Winick, S. Jamin, and J. Rexford. "Traffic engineering between neighboring domains". In: Technical Report (2002) (cit. on pp. 107, 109).

[106] G.J. Woeginger. "On the approximability of average completion time scheduling under precedence constraints". In: Discrete Applied Mathematics 131.1 (2003), pp. 237-252 (cit. on p. 23).

[107] M. Xiao. "Simple and Improved Parameterized Algorithms for Multiterminal Cuts". In: Theory of Computing Systems 46.7 (2010), pp. 723736 (cit. on p. 128). 
[108] Y.R. Yang, H. Wang H. Xie, A. Silberschatz, A. Krishnamurthy, Y. $\mathrm{Liu}$, and E.L. Li. "On route selection for interdomain traffic engineering". In: IEEE Network 19.6 (2005), pp. 20-27 (cit. on p. 109).

[109] D. Zuckerman. "Linear degree extractors and the inapproximability of max clique and chromatic number". In: Theory of Computing 3 (2007), pp. 103-128 (cit. on p. 130). 

In dit proefschrift staan vragen over uitzendschemas en netwerken uit het gebied van combinatorische optimalisatie centraal. Combinatorische optimalisatie is een onderwerp dat bestaat uit het vinden van de beste oplossing uit een eindige verzameling oplossingen. Vrijwel altijd is het een voor een nagaan van alle oplossingen tijdsrovend en praktisch onmogelijk. Daarom is er veel onderzoek gedaan naar betere oplossingsmethodes die efficiënt de beste oplossingen vinden. Echter, er is een grote groep problemen waarvoor er hoogstwaarschijnlijk geen oplossingsmethodes zijn die én efficient zijn én de beste oplossing vinden. Voor deze groep blijkt echter dat het wel vaak mogelijk is om een efficiënte oplossingsmethode te vinden die zeer snel is en een zeer goede oplossing presenteert hoewel dat niet gegarandeerd de bèste oplossing is.

Het eerste deel van dit proefschrift, bestaande uit hoofdstukken 3, 4, 5 en 6 , is gewijd aan vraagstukken die te maken hebben met het efficiënt vinden van uitzendschemas.

In hoofdstuk 4 bestuderen we het probleem Generalized Min Sum Set Cover. Gegeven is een set van elementen $\{1, \ldots, n\}=[n]$ en subsets $S_{1}, \ldots, S_{\mathfrak{m}} \subseteq$ $[\mathrm{n}]$ en een functie $\mathrm{K}:[\mathrm{m}] \rightarrow \mathbb{N}_{+}$. Het doel is om een volgorde $\pi$ van $[\mathrm{n}]$ te vinden zodat de som van raaktijden van de subsets is geminimaliseerd. De raaktijd van een subset $S_{i}$ is gedefiniëerd als het laagste getal $k$ zodat de eerste $k$ elementen van $\pi$ minstens $k\left(S_{i}\right)$ elementen van $S_{i}$ bevatten. In andere woorden: het doel is een volgorde te vinden van elementen zodat de gemiddelde raaktijd van de subsets zo laag mogelijk is.

We definiëren eerst een makkelijker probleem: Preemptive Generalized Min Sum Set Cover, en we presenteren een efficiënte methode gebaseerd op nieuwe inzichten om een 2-approximatie te vinden voor dit probleem. Tevens bewijzen we dat het onwaarschijnlijk is dat er een efficiënte methode is dit probleem beter approximeert, in essentie is ons resultaat het hoogst haalbare. Gebruik makend van onze methode voor Preemptive Generalized Min Sum Set Cover, bewijzen wij een 12.4-approximatie voor Generalized Min Sum Set Cover. 
Hoofdstuk 5 gaat over Minimum Latency Submodular Covering, een zeer algemeen probleem dat bijvoorbeeld Generalized Min Sum Set Cover generaliseert. Gegeven zijn elementen $V$, monotone submodulaire functies $f_{1}, \ldots, f_{m}$ en een afstandsfunctie $d:\left(\begin{array}{l}V \\ 2\end{array}\right) \rightarrow \mathbb{R}_{+}$. Het doel is om een volgorde $\pi=\left(\pi_{1}, \ldots, \pi_{\mathrm{n}}\right)$ van de elementen in $V$ te vinden zodat de raaktijden van de functies $f_{1}, \ldots, f_{m}$ gemiddeld zo laag mogelijk is. De raaktijd van een functie $f_{i}$ is het kleinste getal t zodanig dat $f_{i}(S) \geqslant 1$ waar $S$ de elementen zijn met afstand ten hoogste $t$ in $\pi$.

Wij introduceren dit probleem en laten zien dat het intrinsiek verbonden is met veel bekende problemen, zoals Group Steiner Tree, Submodular Ranking en Set Cover. Naast het interessante gegeven dat het deze problemen verbindt, geven wij een efficiënt $\mathrm{O}\left(\log \frac{1}{\varepsilon} \log ^{2+\delta} n\right)$-approximatie algoritme voor dit probleem, waarin $n$ de instantie grootte is, $\varepsilon$ een technische parameter en voor $\delta$ elk positief getal gekozen kan worden. Dit algoritme en de analyse zijn gebaseerd op een makkelijker bewijs voor Submodular Ranking, ook in dit hoofdstuk.

In hoofdstuk 6 bekijken we de Latency Covering Steiner Tree en Covering Steiner Tree problemen. Voor beide problemen beschouwen we een gewogen graaf $G=(V, E)$ een oorsprong $r \in V$ en groepen $g_{1}, \ldots, g_{k} \subseteq V$ en een bezoekeis voor elke groep $\mathrm{k}:[\mathrm{m}] \rightarrow \mathbb{N}_{+}$. Het doel van Covering Steiner Tree is het vinden van een set van kanten $E^{\prime}$ zodanig dat voor elke groep $g_{i}$ er minstens $k(i)$ knopen verbonden zijn met de oorsprong $r$. Bij het probleem Latency Covering Steiner Tree is er een wederom sprake van het vinden van een volgorde. Het doel is om een route $\pi$ te vinden door de graaf $G$ zodanig dat de som van bezoektijden van alle groepen geminimaliseerd is. De bezoektijd van een groep $g_{i}$ is het eerste tijdstip zodanig dat de route minstens $k(i)$ knopen van $g_{i}$ heeft bezocht.

De huidige oplossing voor Covering Steiner Tree bestaat uit het herhaaldelijk oplossing van een linear programma, wat een kostbare stap is. Wij formuleren een nieuw linear programma dat slechts eenmalig opgelost hoeft te worden. Gebaseerd op dit nieuwe lineare programma ontwikkelen we een nieuw $\mathrm{O}\left(\log ^{2} \mathrm{n}\right)$-approximatie algoritme voor Latency Covering Steiner Tree, waarin $n$ de instantie grootte is.

Het tweede deel van dit proefschrift bestaat uit hoofdstukken 7 en 8 .

Hoofdstuk 7 bestudeert een probleem rondom het versturen van datastromen op het internet. Het internet is niet één geheel, maar een netwerken van verscheidene verbonden subnetwerken met elk een andere eigenaar. In een netwerk, of graaf, $G=\left(V_{1}, V_{2}, E\right)$ zijn er twee subnetwerk eigenaars waarvan een $V_{1}$ bezit en de ander $V_{2}$. Elk subnetwerk bevat gebruikers die 
een bericht willen sturen aan gebruikers in het andere subnetwerk. Voor elk bericht moet er een route gevonden worden door beide subnetwerken en het liefst een zo goedkoop mogelijke route voor de eigenaars. Op de rand tussen de subnetwerken wordt een bericht overgedragen, en de andere eigenaar zorgt voor de bezorging. Normaal gesproken beperkt elke eigenaar zich tot zijn eigen netwerk: het bericht leveren ze af op een willeurige plek waar beide subnetwerken verbonden zijn. Dit hoofdstuk bestudeert of er samenwerking mogelijk is bij het overdragen van berichten tussen de verschillende subnetwerken, zodat voor elk daarvan de kosten afnemen.

Wij laten zien dat er een zeer snel praktisch algoritme is dat de beste samenwerking tussen twee netwerken bepaalt. Naast experimenteel bewijs, geven wij ook een wiskundig bewijs dat dit algoritme efficiënt is, door middel van smoothed analysis.

Het laatste hoofdstuk 8 gaat over het probleem k-Way Vertex Cut. In dit probleem is het doel om een groep knooppunten in een netwerk te vinden, zodanig dat het verwijderen van deze knooppunten het netwerk opsplitst in veel onafhankelijke stukken. Voor planaire netwerken presenteren wij een Efficient Polynomial Time Approximation Scheme. Daarnaast bestuderen wij de complexiteit van dit probleem op verscheidene interessante graaf klassen, zoals interval graphs, split graphs en graphs of bounded treewidth. 

Gijsbert Ruben Johan van der Zwaan was born on April 8, 1985 in Utrecht, The Netherlands. In 2003, he received his VWO diploma at Montesorri Lyceum Herman Jordan in Zeist. In September of the same year he started studying Computer Science at Utrecht University. He received his Master's degree early 2009. From May 2009 until May 2012 Ruben was employed as a PhD student in the Operations Research group in the Quantitative Economics department of Maastricht University. Part of the results of his research are presented in this thesis. 



\section{ACKNOWLEDGEMENTS}

My expectation is that while reading this sentence, your first thought is that it is different from any other acknowledgement section you might have read. Although the reason behind a different beginning will become clear later, let me assure you that the story has a happy ending. But, for now, please permit me to be a little different; be patient with me pondering the rationale of "The Acknowledgement Section".

It all started a very long time ago, in a place far, far away. I was happy and oblivious to the world of acknowledgments, although I had much to be thankful for. This all changed when I started this thesis and slowly but surely I thought more and more about the writing of my own thesis. I wish to impress on you that it was nothing obsessive, lest you think I contemplate about such things daily.

I readily admit that when reading the section "Acknowledgements" in other theses or books for the first time, I assumed that I would not engage in such frivolous use of paper. What did their parents, friends or readers like you, contribute? Which page? What sentence? Not that I do not appreciate you, but really, it is a thing between us: a personal "thank you" is much more appropriate.

However, as time passed as a $\mathrm{PhD}$ student I realized that my personal life and my work - this thesis - were horribly intertwined! The first time this thought dawned on me, I was appalled: am I that obsessed with my work?! The issue of my obsession aside, I can honestly say that this work would be different without you. This thesis is more than a collection of my writings. For good or bad, it is three years of my life and therefore, for me, it is permeated with emotions and memories. Luckily, the last three years were fantastic, and there was much more good than bad. Therefore, I hope that when you read this thesis you see more than the, perhaps incomprehensible, mathematical proofs or dry text-walls of doom.

So, here it is, the promised happy ending, after only three and a half paragraphs. You are about to be thanked!

This thesis would not exist if not for André: I could not have wished for a better supervisor. 
The enthusiasm of Alexander Grigoriev made my time in Maastricht enjoyable, I am still wondering how he got work done with the amount of visits I payed him. Many thanks to Maxim Sviridenko for hosting me in the summer of 2012 at IBM in New York and I am thankful for the collaboration with him, Sungjin Im and Viswanath Nagarajan. Already before I was a PhD student, I had the pleasure of meeting Pinar Heggernes and luckily I was able to have another wonderful visit to Bergen last autumn. Jesper Nederlof has been listening to my ideas/thoughts for a very long time, and I am happy that one of these discussions led to a project together with him and Erik Jan van Leeuwen. Although Heiko Röglin's stay in Maastricht was short, it was definitely memorable. Sebastián Marbán and Tjark Vredeveld are thanked for the pricing paper, we (again) learned that the devil is in the details.

While I did spend quite some time in the offices of other people, I am very happy with my own lair and the people I share(d) it with. Daily raiding for supplies is made more fun, and there were many philosophical discussions of which some are still ongoing.

Finally, I thank my promotor Stan van Hoesel and all the people at Quantitative Economics: it was (and still is) a joy to work there, thank you!

I sincerely enjoyed the teaching and luckily I am doing that. Almost always, in my experience, students are engaging and eager to learn. This easily counteracts the boring parts - for example having to repeat the same lecture four times on a day.

Being taught by me is not always a bitter pill for students, sometimes life is sweet. Handing out candy as a bonus for correct answers works, but having students hand each other candy for correct answers works better, and is considerably cheaper. Strangely enough, I still get credited for the candy!

Rather than enumerating the names of people I am especially thankful to, I rather describe them or events I associate with them. I am sure that you can recognize yourself when I am referring to you. If you wonder where your "thank you" is: well it is here, just look harder!

From Magyarország, the Hunter of Maastricht, who sometimes comes back empty-handed and eats salmon like clockwork. Also from that lovely part of the world: the old man from the gym with heart problems during coffee breaks. I've never seen such local attacks of hay fever and intense staring. I miss the running and excited sounds of El Matador in the hallways, although if he did not come knocking on my door I got more work done. As a side remark: he also suffered from the local waves of being 
distracted during coffee breaks. Liverpool reminds me of my liver thanks to a certain person, and I am sure the sun was brighter than usual the next day. Wednesdays are also known as "Dynamic Duo Power Days" to those that need coffee or tea. They are like the "Power Rangers", fearsome on their own but terrifying when they combine their "Zords" to make a "Mega-super-duper-Zord". Note that I did not make up the word "Zord".

My paranimfs, who were selected for their special qualities. Making an foldable and wearable fake-guitar to play on and that suits your clothing is awesome, I hope more inventions are on their way. The fact that I consider inventing such stuff as completely natural perhaps indicates for how long I know my paranimf. Being my brother is indeed special and unique, and no one could do what he does nor could I wish for a better brother. I am sure these qualities will be essential during my defense.

My parents and my brother whom I love very much and I am thankful to have them. My girlfriend, who makes me smile and whom I like better than a cheese sandwich. My friends and family have been invaluable and provided necessary and unnecessary distractions.

There are several things and topics that I learned about in the last three years (in no particular order): coffee breaks, coffee in church, milk in coffee, online reconnaissance, salsa, telephone conversations, ants, sleeping in planes, Mexican food and drinks, evasive driving after said Mexican food and drinks, dromedarii smell badly, airports are still boring, presenting at a conference can be fun, explain first why your research is important before you explain why the technical difficulties make you awesome (or not), evading work is difficult if you only need pen and paper, knocking on doors is fun, speaking Latin is sexy for laboratory people, Pavlov training works well on children, stress is bad. 